DEPARTMENT OF THE INTERIOR

UNITED STATES GEOLOGICAL SURVEY 、

CHARLES D. WALCOTT, DIRECTOR

\title{
MINERAL ANALYSES
}

FROM THE

\section{LABORATORIES OF THE INITED STATES GEOLOGICAL SURVEI}

\section{0 to 1903}

TABULATED BY

F. W. CLARKE, Chief Chemist

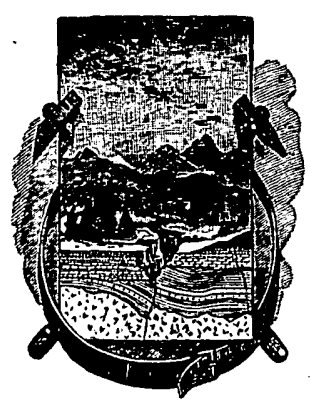

W A S HIN GTON

GOVERNMENT PRINTING OFFICE

1903 
$5 . \sigma_{0}$.

Q E is

B9

ro.220
oopy 3 


\section{0 N T E N T S.}

Letter of transmittal, by George F. Becker

Introduction.

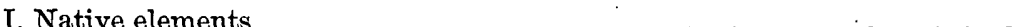

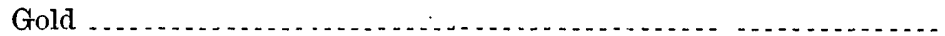

Josephinite

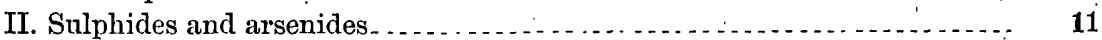

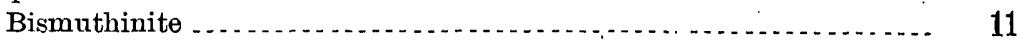

Metacinnabarite $\ldots \ldots \ldots \ldots . . .11$

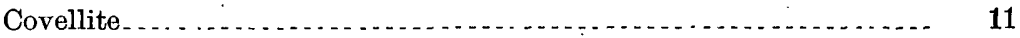

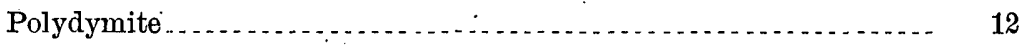

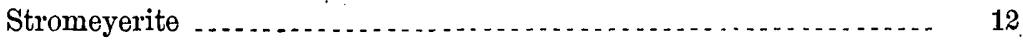

Sulphide of silver, copper, and zinc........................ 12

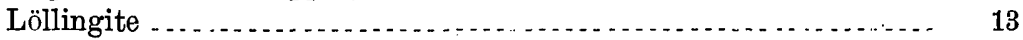

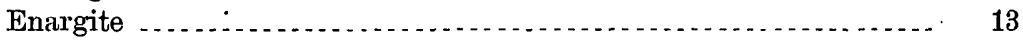

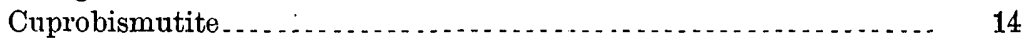

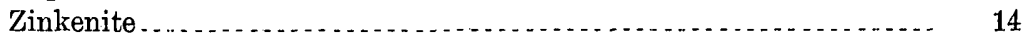

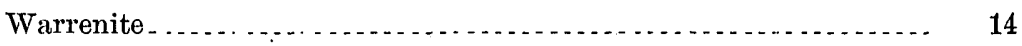

Cosalite . . . . .

Freieslebenite -

Guitermanite .....................

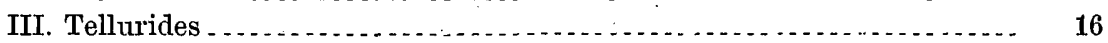

Hessite $\ldots \ldots \ldots \ldots$

Petzite $\ldots \ldots \ldots$

Calaverite $\ldots . . .16$

Melonite $\ldots . . . . . . . .17$

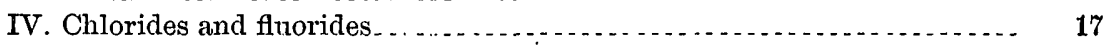

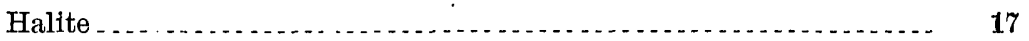

Embolite

Cryolite ....... . . . . . 17

Pachnolite. . . . .

Elpasolite $\ldots \ldots \ldots$

Gearksutite $\ldots \ldots \ldots \ldots \ldots$

Prosopite . .

Tysonite . . . . 19

V. Oxides and hydroxides ....................................... 20

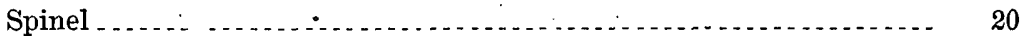

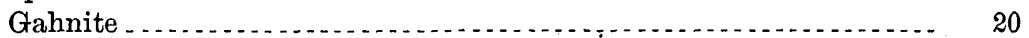

Magnetite . . . . .

Chromite ...

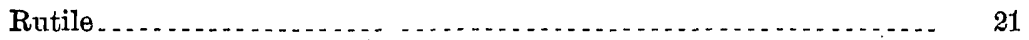

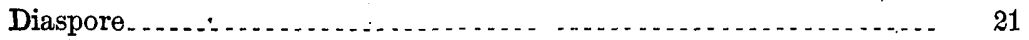

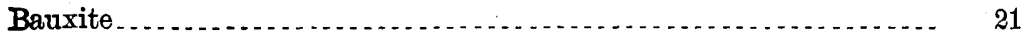

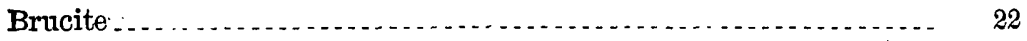

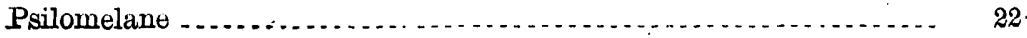

○. 3 
VI. Carbonates

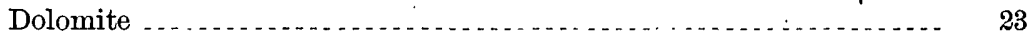

Smithsonite. . .

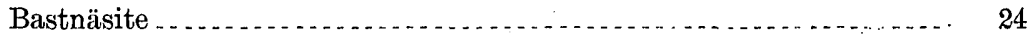

Urao

VII. Silicates....

Petalite ...

Orthoclase and microcline.................................. 25

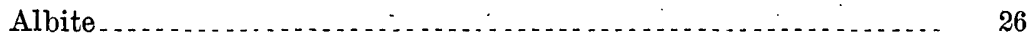

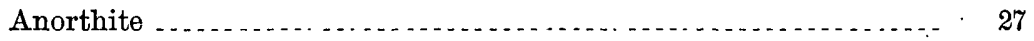

Soda-lime feldspars

Leucite

Enstatite ...

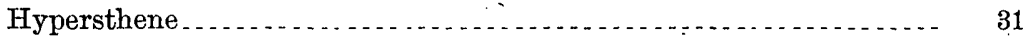

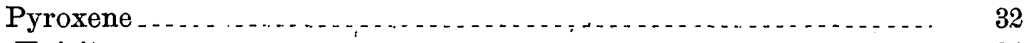

Agirite

Jadeite

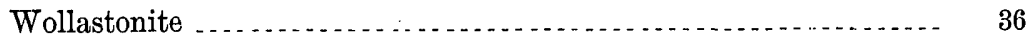

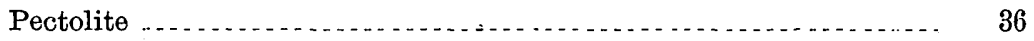

Amphibole

Beryl

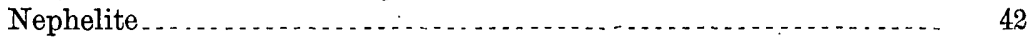

Cancrinite

Sodalite

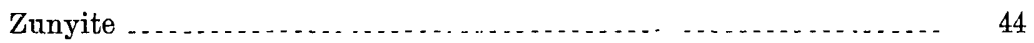

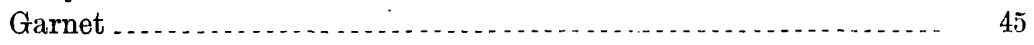

Chrysolite . . . . .

Mizzonite....

Vesuvianite . .

Zoisite .

Epidote $\ldots \ldots$

Piedmontite .............. 50

Allanite

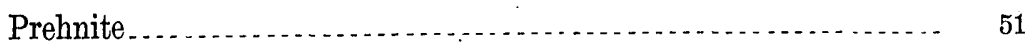

Topaz

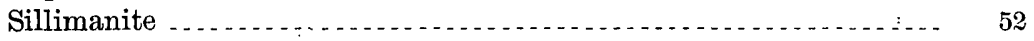

Kyanite

Ivaite...

Calamine .....

Lawsonite $\ldots \ldots$

Staurolite ............... 54

Gadolinite

Yttrialite . . .

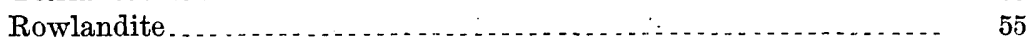

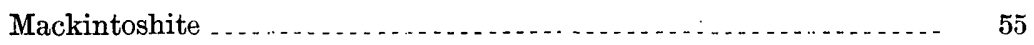

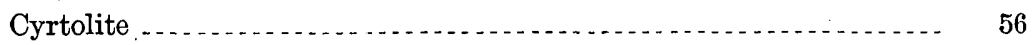

Danburite ................. 57

Datolite

Axinite

Dumortierite

Tourmaline.............. 58

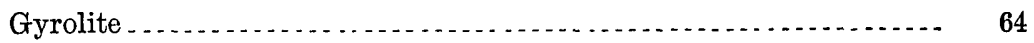


VII. Silicates-Continued.

Page.

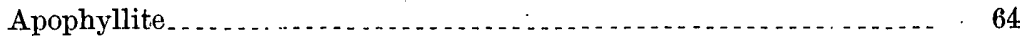

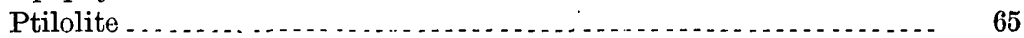

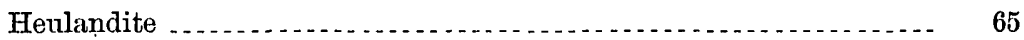

Stilbite

Laumontite $\ldots \ldots \ldots 6 \ldots$

Chabazite $\ldots \ldots \ldots 6$. 67

Levynite _....

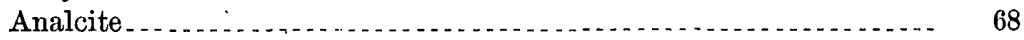

Natrolite

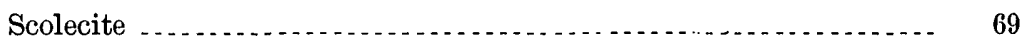

Mesolite _... 69

Thomsonite $\ldots \ldots \ldots$

Hydronephelite $\ldots \ldots$

Muscovite . . . . .

Lepidolite $\ldots \ldots \ldots \ldots$

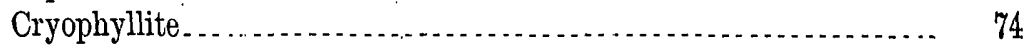

Biotite ... . . . . . .

Phlogopite $\ldots \ldots$

Lepidomelene. . . . .

Roscoelite .......

Margarite _..

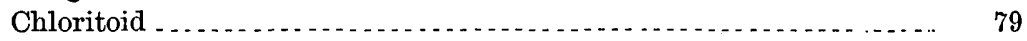

Xanthophyllite _...

The vermiculites. $\ldots \ldots \ldots$

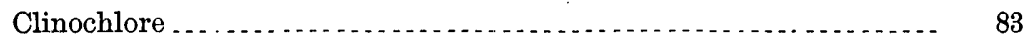

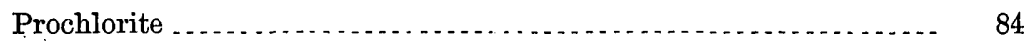

Serpentine $\ldots \ldots \ldots \ldots$

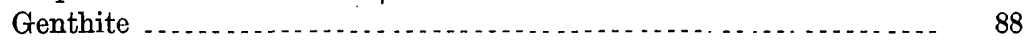

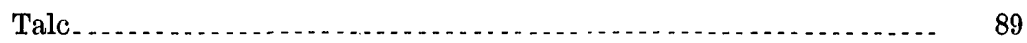

Glauconite $\ldots \ldots \ldots \ldots$

Kaolinite $\ldots \ldots \ldots \ldots$

Halloysite $\ldots \ldots \ldots \ldots \ldots$

Cimolite . .

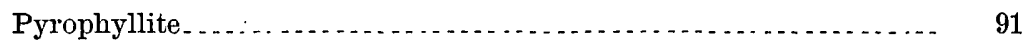

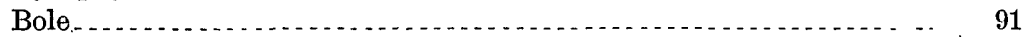

VIII. Titanates and titano-silicate $\ldots \ldots \ldots \ldots \ldots \ldots \ldots$

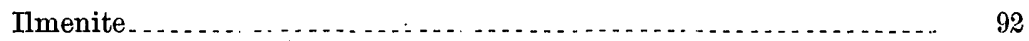

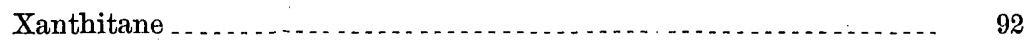

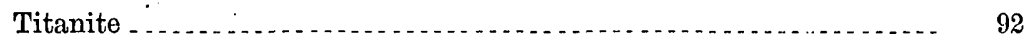

Tscheffkinite $\ldots \ldots 3$

Astrophyllite

IX. Columbo-tantalates :

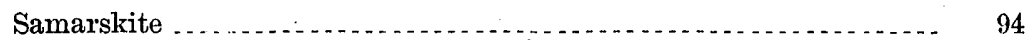

X. Evrates _...

Colemanite

Ulexite....

Ludwigite $\ldots \ldots \ldots \ldots$

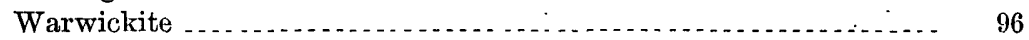

XI. Nitrates $\ldots \ldots \ldots 6$

Soda niter $\ldots \ldots 6$

Niter. 


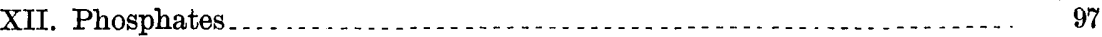

Xenotime

Apatite

Triplite ...

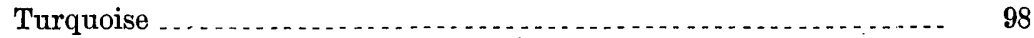

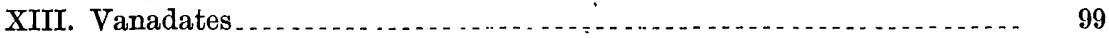

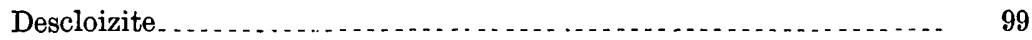

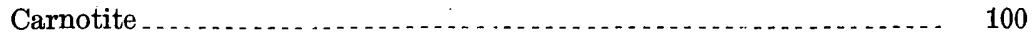

XIV. Arsenites and antimonites................................... 101

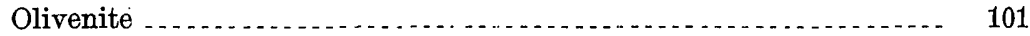

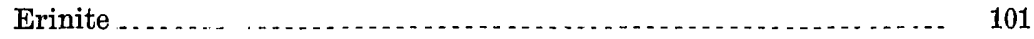

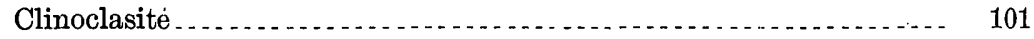

Conichalcite . . . . . . 102

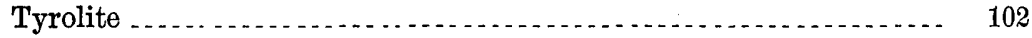

Chenevixite $\ldots 2$

Mixite

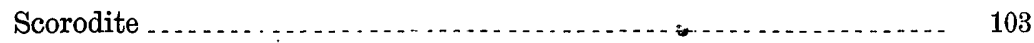

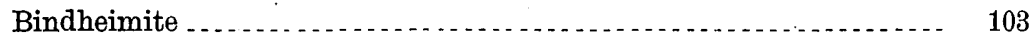

XV. Sulphates and tellurites ............................................ 104

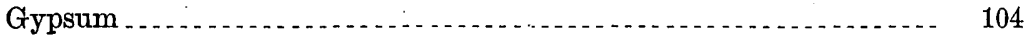

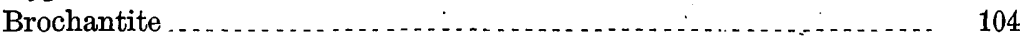

Antlerite . . . . . . . . . . . . 105

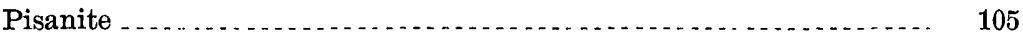

Picrallamogene ...

Halotrichite . . . . . 106

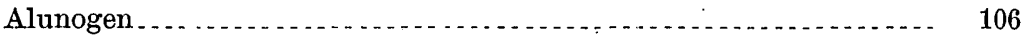

Copiapite ... . .

Knoxvillite ... . . . . . . 107

Redingtonite . . . . . . . . . 107

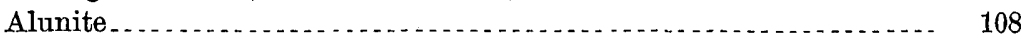

Jarosite .... . . . . . . 108

Natrojarosite

Plumbojarosite . . . . . 109

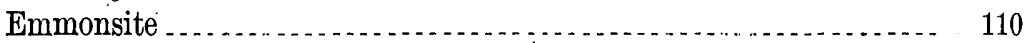

XVI. Molybdates, tungstates, and uranates ................. 110

Powellite . . . . . . . 110

Hübnerite $\ldots . \ldots \ldots \ldots \ldots$

Uraninite ... . . . 111

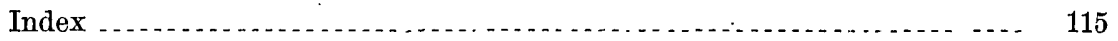




\title{
LETTER OF TRANSMITTAL.
}

\author{
DEPARTMENT OF THE INTERTOR, \\ United States Geological Survey, \\ Washington, D. C., October 8, 1908.
}

STR: I have the honor to transmit herewith a paper entitled "Mineral Analyses from the Laboratories of the United States Geological Survey, 1880-1903, tabulated by T. W. Clarke, Chief Chemist." Much of the material of this paper is unpublished, and it is very desirable that the whole should be printed in a conveniently accessible form.

Very respectfully,

GEORGE F. BECKER,

Geologist in Charge, Division of Physical and Chemical Research.

Hon. Chardes D. Walcott, Director United States Geological Survey. 



\section{MINERAL ANALYSES FROII LABORLTORIES OF UNIEED STATES GEOLOGICAL SURVEY, 1880-1903.}

Tabulated by F. W. ClankE, Chief Chemist.

\section{INTRODUCTION.}

In the present bulletin I have brought together and classified 507 analyses of minerals from the laboratory records of the United States Geological Survey. A large proportion of these analyses have been already published elsewhere, but they are so scattered that their collection in one place seemed to be desirable. Over 150 distinct species are represented, and among them a considerable number were originally described by chemists connected with the Survey. These species are josephinite, cuprobismutite, warrenite, guitermanite, elpasolite, zunyite, ptilolite, hydronephelite, lucasite, antlerite, knoxvillite, redingtonite, plumbojarosite, emmonsite, and powellite. In other cases minerals which were imperfectly described have been more sharply characterized and their true composition made known. Natrojarosite, for example, is here definitely recognized as a species.

In general the order of Dana's classification has been followed, but with some small variations. Thus the tellurides are put in a group by themselves; the borosilicates are brought together, while the phosphates, vanadates, and arsenates are given as three separate classes. On purely chemical grounds these changes are warranted; on morphological grounds the usual mineralogical classification may be better.

Detailed descriptions of the various minerals have been purposely omitted. Neither are the analyses discussed. These deficiencies, however, are mitigated by the literature references to descriptions published elsewhere. Such references as " annual report," "bulletin," or "monograph" relate to the regular publications of this Survey; all other references are easily recognizable. 


\section{NATIVE ELEMENTS.}

GOLD.

From Persia; exact locality unknown. Analyzed by Charles Catiett.

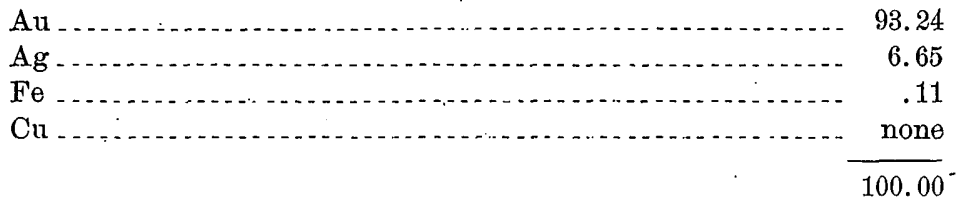

JOSEPHINITE.

A nickel-iron alloy found in placer gravels in Jacksqn and Jósephine counties, Oreg. Described as a new species by W. H. Melvilie, Am. Jour. Sci., 3d ser., vol. 43, 1892, p. 509. Waterworn pebbles. Specific gravity, 6.204 .

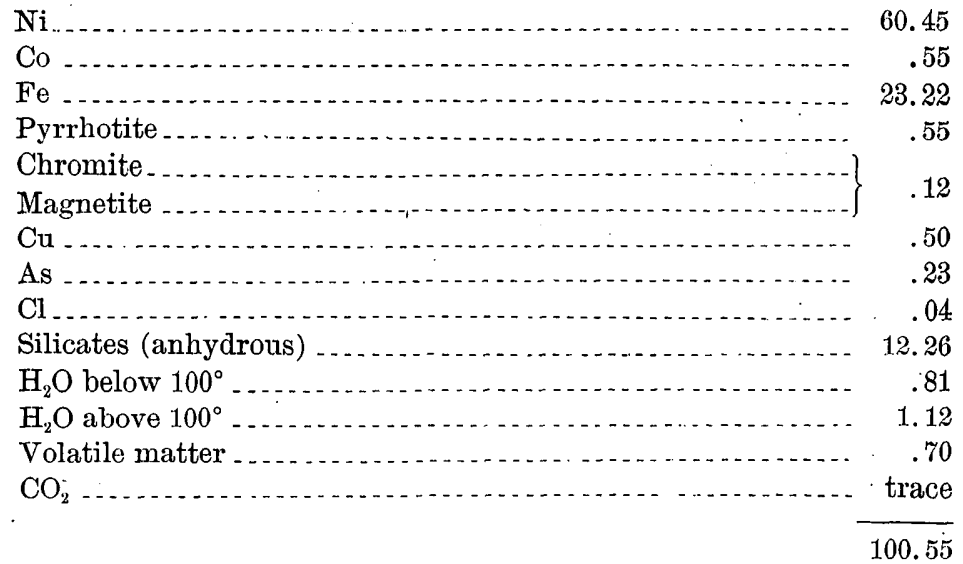

The silicate admixture, including the water, amounts to 13.38 per cent, of which 12.88 per cent, soluble in hydrochloric acid, is serpentine. The insoluble portion may be bronzite. Analyses of the silicates are as follows:

\begin{tabular}{|c|c|c|c|}
\hline & Total. & $\begin{array}{l}\text { Insoluble } \\
\text { portion. }\end{array}$ & $\begin{array}{l}\text { Soluble } \\
\text { portion. }\end{array}$ \\
\hline $\mathrm{SiO}_{2} \ldots \ldots$ & 5.14 & 0.23 & 4.91 \\
\hline $\mathrm{Al}_{2} \mathrm{O}_{3}$ & .33 & .03 & .30 \\
\hline $\mathrm{Fe}_{2} \mathrm{O}_{3}$ & 2.08 & .04 & 2.04 \\
\hline $\mathrm{NiO}, \mathrm{CoO} \ldots$ & .32 & trace & .32 \\
\hline $\mathrm{CaO} \ldots \ldots . . . . .$. & 1.62 & .06 & 1.56 \\
\hline $\mathrm{MgO}$ & 2.69 & .14 & 2.55 \\
\hline $\mathrm{Na}_{2} \mathrm{O}$ & .08 & & .08 \\
\hline $\mathrm{H}_{2} \mathrm{O}$ above $100^{\circ} \ldots$ & 1.12 & - & 1.12 \\
\hline . & 13.38 & .50 & 12.88 \\
\hline
\end{tabular}

For analyses of nickel-iron of meteoric origin, see Bulletin 168. 


\section{SULPHIDES AND ARSENIDES.}

\section{BISMUTHINITE.}

From the Rosario Mining District, Sinaloa, Mexico. Analysis by W. H. Melville. Described by him in Bulletin 90 .

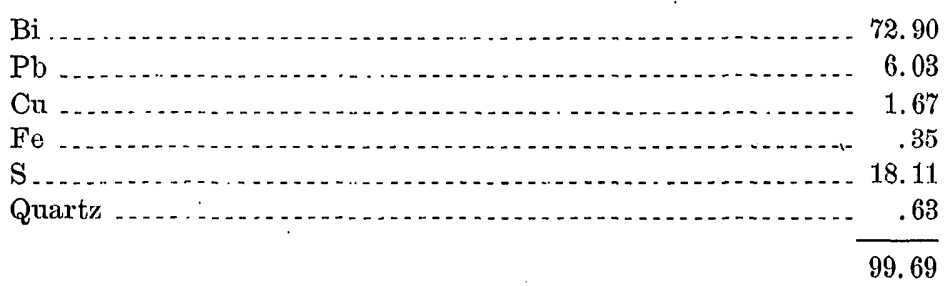

METACINNABARITE.

Crystallized material from New Almaden, Cal. Analysis by W. H. Melville. Not free from admixtures.

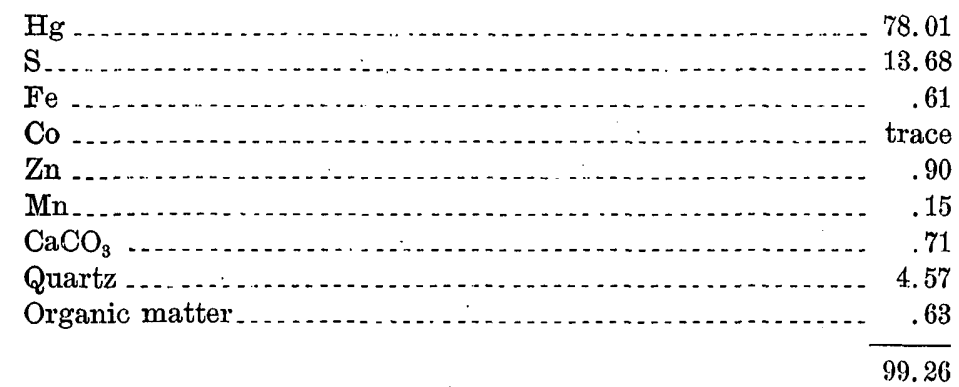

Another specimen of metacinnabarite from Knoxville, Cal., gave Melville the following figures:

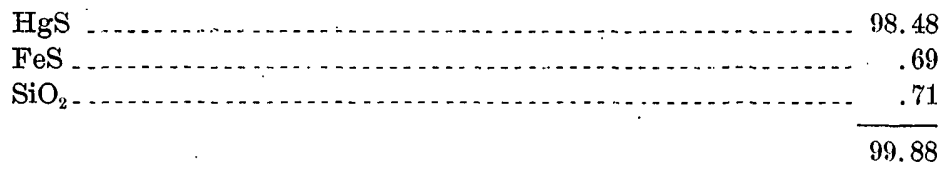

The latter mineral was described by Melville and Lindgren. in Bulletin 61.

\section{COVELIITE.}

From the Eaist Greyrock mine, Butte, Mont. Collected by G. W. Tower. Analysis by W. F. Hillebrand. Color, indigo-blue. Massive. Specific gravity, 4.76 at $26^{\circ}$.

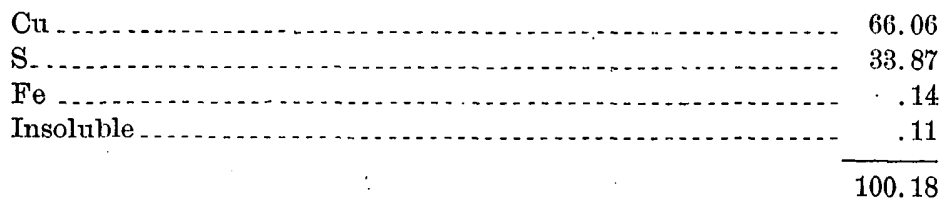




\section{POLYDYMITE.}

A massive ore from the mine of the Canadian Copper Company, Sudbury, district of Algoma, Ontario. Specific gravity, 4.541. Analysis by Charles Catlett. Described by Clarke and Catlett in Am. Jour. Sci., 3d ser., vol. 37,1889 , p. 372 . Composition nearly $\mathrm{Ni}_{3} \mathrm{FeS}_{5}$.

A. Actual analysis.

B. Analysis corrected by deduction of quartz and chalcopyrite.

\begin{tabular}{|c|c|c|}
\hline & A. & B. \\
\hline $\mathrm{Ni} \ldots$ & 41.96 & 43.18 \\
\hline $\mathrm{Fe} \ldots \ldots$. & 15.57 & 15.47 \\
\hline S & 40.80 & 41.35 \\
\hline $\mathrm{Cu} \ldots \ldots \ldots \ldots$ & .62 & $\ldots$ \\
\hline \multirow[t]{2}{*}{$\mathrm{SiO}_{2}$} & 1.02 & $\ldots$ \\
\hline & 99.97 & 100.00 \\
\hline
\end{tabular}

Another nickel-iron sulphide, from the Worthington mine, Sault branch of the Canadian Pacific Railway, 25 miles west of Sudbury, has been analyzed by W. F. Hillebrand. Grayish, with a cast of yellow. Not pyrrhotite. Possibly a mixture of polydymite and pyrite.

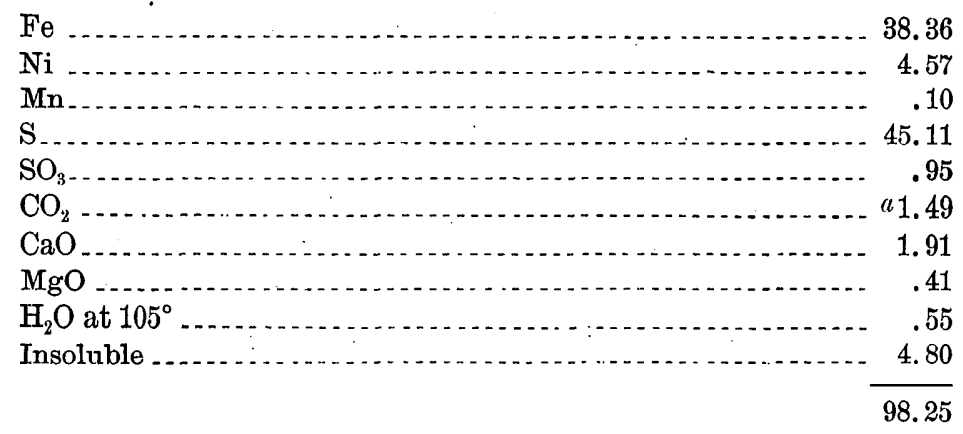

STROMEYERITE.

From the Silver King mine, Calico, San Bernardino County, Cal. Specific gravity, 6:28. Analysis by W. H. Melville. Described by Melville and Lindgren in Bulletin 61.

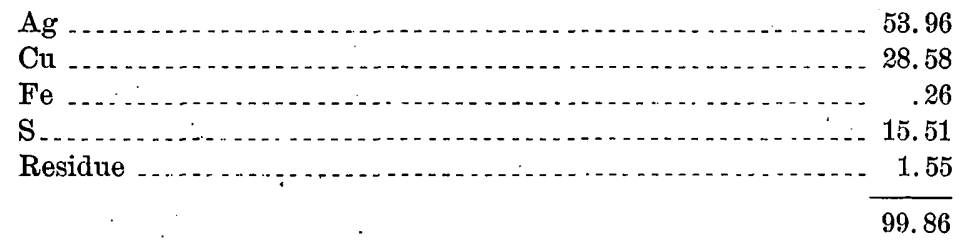

SULPHIDE OF SILVER, COPPER, AND ZINC.

Massive, resembling bornite. Apparently homogeneous, but may be a mixture. Specific gravity, 5.407 at $20^{\circ}$. Analyzed by W. F. 
Hillebrand and described in Bulletin 55. From the Gagnon mine, Butte, Mont.

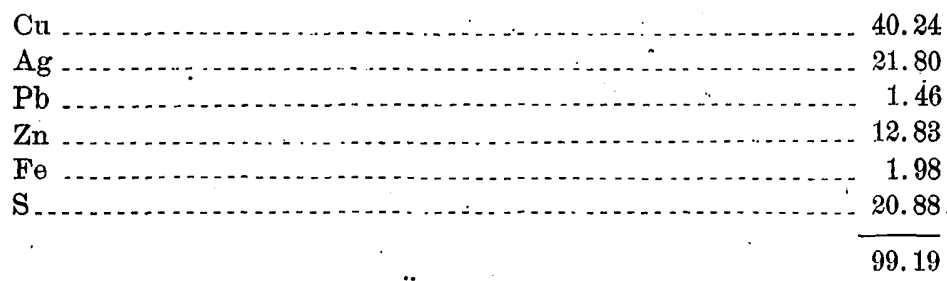

\section{LÖLLINGITE.}

From Teocalli Mountain, Brush Creek, Gunnison County, ('olo. Specific gravity, 7.400 at $14.5^{\circ}$, corrected for impurity. Analyzed by W. F. Hillebrand and described in Bulletin 20.

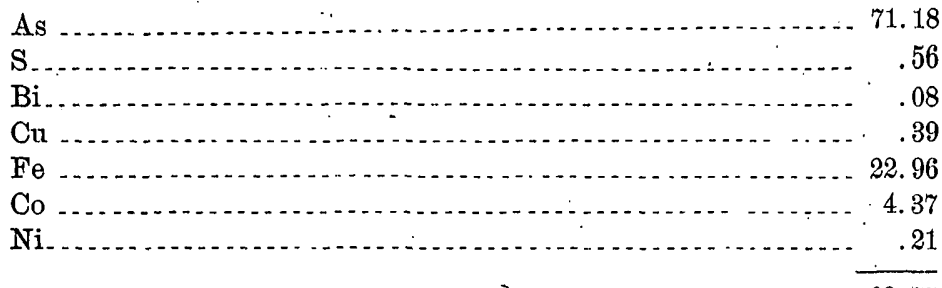

99.75

A doubtful arsenide of nickel and cobalt has also been examined by Hillebrand and described in Proc. Colorado Sci. Soc., vol. 3, pt. 1, p. 46. From the Rose mine, Grant County, N. Mex. Specific gravity, 6.644 at $20^{\circ}$. Probably a mixture. Ni: $\mathrm{Co}=3: 1$, approximately.

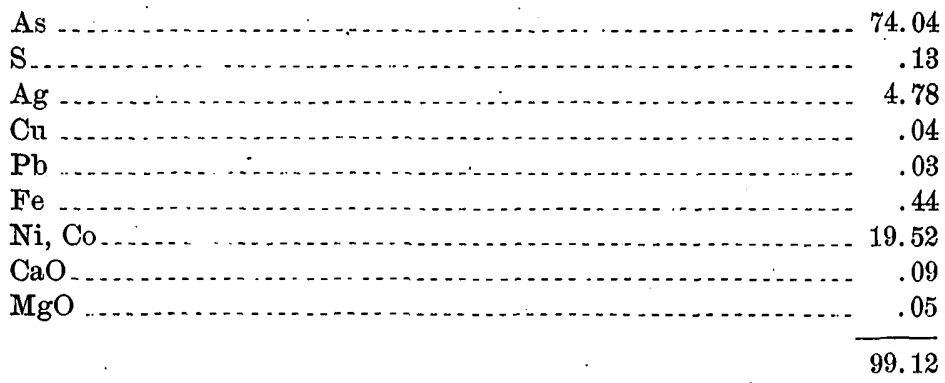

ENARGITE.

From the Rarus mine, Butte, Mont. Collected by G. W. Tower. Analysis by W. F. Hillebrand.

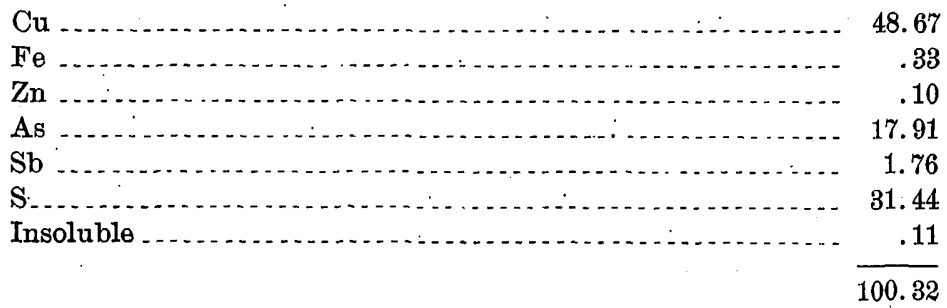




\section{CUPROBISMUTITE.}

New species, discovered by W. F. Hillebrand and described by him in Bulletin 20. Named by Dana. Specific gravity, 6.680 at $15^{\circ}$, corrected for impurities. From the Missouri mine, Halls Valley, Park County, Colo. Analyses, by Hillebrand, of three different samples.

\begin{tabular}{|c|c|c|c|}
\hline & I. & II. & III. \\
\hline $\mathrm{Bi}$ & 60.74 & 63.36 & 62.45 \\
\hline Ag ... & .89 & 4.09 & 9.89 \\
\hline $\mathrm{Cu} \ldots \ldots$ & 15.96 & 12.65 & 6.68 \\
\hline $\mathrm{Pb}$ & & & 2.74 \\
\hline $\mathrm{Fe} \ldots$ & 2.13 & .59 & .10 \\
\hline $\mathrm{Zn} \ldots \ldots$ & .10 & .07 & .07 \\
\hline \multirow[t]{2}{*}{ S } & «19.94 & a 18.83 & 17.90 \\
\hline & 99.76 & 99.59 & 99.83 \\
\hline
\end{tabular}

$a$ Calculated.

ZINKENITE.

From the Brobdignag mine, Red Mountain, San Juan County, Colo. Specific gravity, 5.21 at $18^{\circ}$. Analyzed by W. F. Hillebrand and described by him in Bulletin 20.

\begin{tabular}{|c|c|c|}
\hline & I. & II: \\
\hline $\mathrm{Sb} \ldots$ & 35.00 & \\
\hline As $\ldots \ldots \ldots$ & 5.64 & 5.59 \\
\hline $\mathrm{Pb} \ldots \ldots$ & 32.77 & 32.79 \\
\hline $\mathrm{Cu} \ldots$ & 1.20 & $\ldots$ \\
\hline $\mathrm{Ag}$ & .23 & $-\ldots$ \\
\hline $\mathrm{Fe} \ldots \ldots$ & .02 & 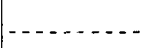 \\
\hline $\mathrm{CaO}$ & .31 & $\ldots$ \\
\hline Alkalies . . . . . . . . . & .45 & ..... \\
\hline $\mathbf{S}_{\ldots} \ldots \ldots \ldots \ldots$ & 22.50 & 22.50 \\
\hline \multirow[t]{2}{*}{ Gangue $\ldots \ldots \ldots \ldots$} & .59 & (n..... \\
\hline & 98.71 & $\cdots$ \\
\hline
\end{tabular}

\section{WARRENITE.}

New species, described by L. G. Eakins in Am. Jour. Sci., 3d ser., vol. 36, 1888, p. 450. From the Domingo mine, Gunnison County, Colo. Occurs in matted, fibrous masses; known locally as "mineral wool." Analysis by Eakins. 
$\mathrm{Pb}$ 39.33

$\mathrm{Sb}$ 36.34

$\mathrm{S}$ 21.19

Ag . . . . . .

$\mathrm{Fe} \ldots \ldots \ldots \ldots$

Mn . . . . . . . . . . . . . .

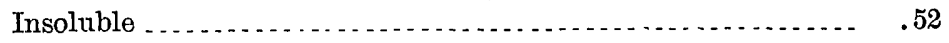

COSALITE.

99.15

From the Comstock mine, near Parrott City, La Plata County, Colo. Described by W. F. Hillebrand in Bulletin 20.

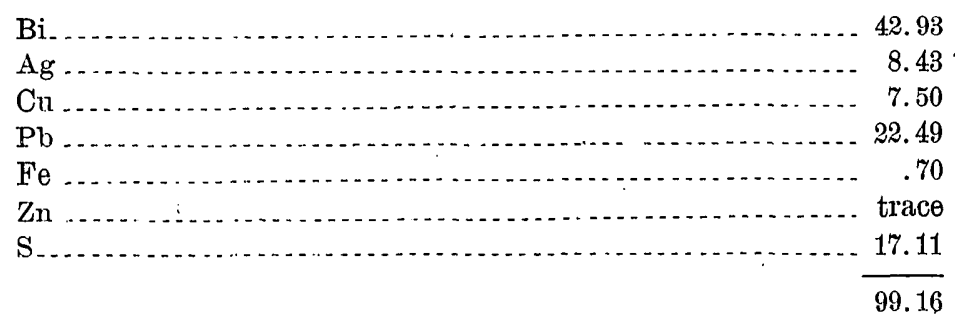

FREIESLEBENITE.

From Augusta Mountain, Gunnison County, Colo. Known locally as "mineral wool." Remarkable for its freedom from silver. Analyzed by L. G. Eakins and described by him in Am. Jour. Sci., 3d ser., vol. 36,1888 , p. 452 .

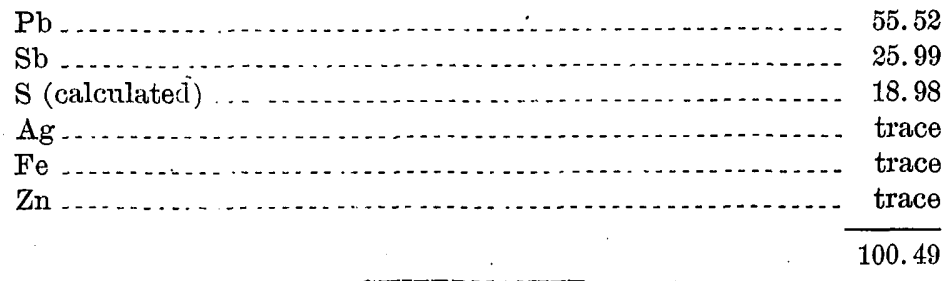

GUITERMANITE.

New species, discovered by W. F. Hillebrand and described by him in Bulletin 20. From the Zuñi mine, Anvil Mountain, near Silverton, San Juan County, Colo. Forms the matrix of zunyite. Corrected specific gravity, 5.94 at $17.5^{\circ}$. Analyses by Hillebrand.

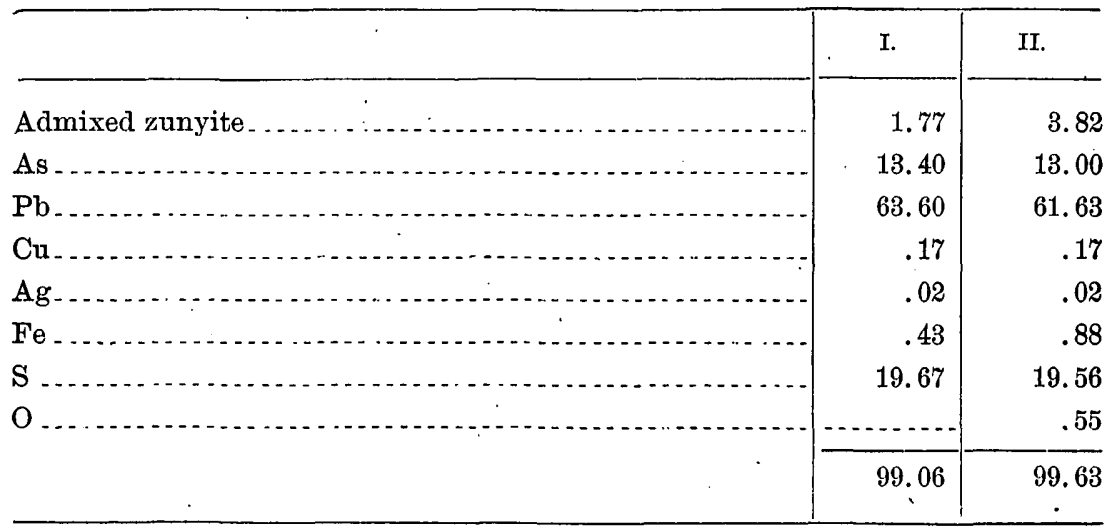




\section{TELLUR'IDES.}

HESSITE.

From San Sebastian, Jalisco, Mexico. Specific gravity, 8.24 at $26^{\circ}$. Analysis by W. F. Hillebrand.

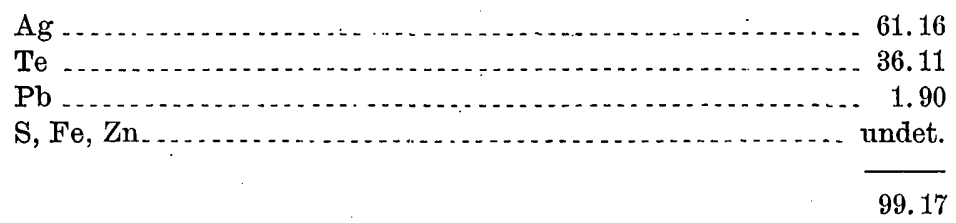

PETZITE.

From the Norwegian mine, Calaveras County, Cal. Collected by F. I. Ransome. Specific gravity, 8.925 at $23^{\circ}$. Analysis by W. F. Hillebrand. Formula, $\mathrm{Au}_{2} \mathrm{Te} \cdot 3 \mathrm{Ag}_{2} \mathrm{Te}$.

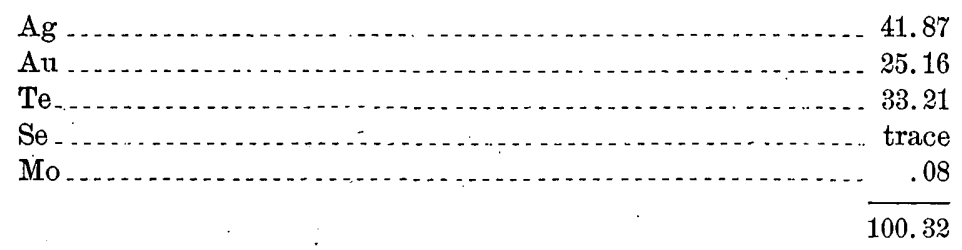

\section{CALAVERITE.}

From Cripple Creek, Colo. Collected by R. A. F. Penrose, jr. Analyzed by W. F. Hillebrand and described by him in Bulletin 167.

A. From the Prince Albert mine. Corrected specific gravity, 9.0 at $24^{\circ}$.

B. Raven mine.

C. C. O. D. mine.

\begin{tabular}{|c|c|c|c|}
\hline$\therefore$ & A. & B. & $\mathrm{C}$ \\
\hline Te $\ldots \ldots \ldots$ in & 57.27 & 47.69 & 53.89 \\
\hline Aun $\ldots \ldots \ldots \ldots \ldots$ & 38.95 & 33.93 & 39.31 \\
\hline Ag $\ldots \ldots \ldots$ & 3.21 & 1.47 & .85 \\
\hline Insoluble matter & .33 & 5.80 & .91 \\
\hline $\mathrm{Fe}_{2} \mathrm{O}_{3} \ldots \ldots$ & .12 & $\ldots .$. & ...- \\
\hline $\mathrm{Fe} \ldots \ldots \ldots$ & $\ldots$ & 5.41 & 1.67 \\
\hline$S_{2} \ldots \ldots \ldots$ & 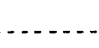 & $a 6.17$ & 1.58 \\
\hline Mn $\ldots \ldots \ldots$ & & $\ldots . .$. & $b .23$ \\
\hline $\mathrm{Ca} \ldots \ldots \ldots \ldots \ldots$ & & & .51 \\
\hline Mg & ..... & $\cdots$ & .10 \\
\hline \multirow[t]{2}{*}{ 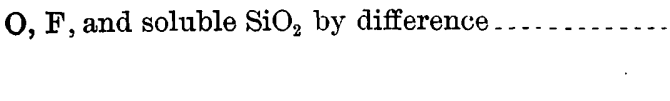 } & & $\ldots$ & .95 \\
\hline & 99.88 & 100.47 & 100.00 \\
\hline
\end{tabular}


MELONITE.

From the Melones mine, Carson Hill, Calaveras County, Cal. Collected by F. L. Ransome. Analyzed by W. F. Hillebrand and described by him in Bulletin 167. Three samples. Specific gravity of $\mathrm{B}, 7.72$ at $22.5^{\circ}$, which is probably too high for the pure $\mathrm{NiTe}_{2}$. Sample C is the purest.

\begin{tabular}{|c|c|c|c|}
\hline . & A. & B. & C. \\
\hline $\mathrm{Te}$ & 75.29 & 77.72 & 80.75 \\
\hline $\mathrm{Ni}$ & 15.71 & 17.16 & 18.31 \\
\hline Co ....... & & .10 & \\
\hline $\mathrm{Ag}$ & 8.44 & 5.09 & .86 \\
\hline$\cdot$ & 99.44 & 100.07 & 99.92 \\
\hline
\end{tabular}

\section{CHLORIDES AND FLÚORIDES.}

HALITÉ.

Rock salt from Salton, Cal. Analysis by E. T. Allen.

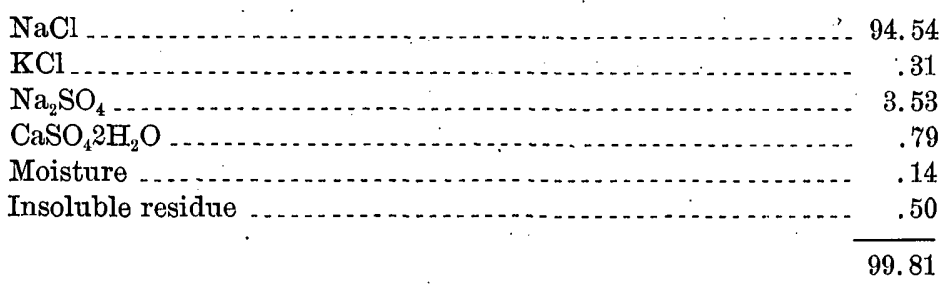

EMBOLITE.

From Broken Hill, Australia. Analysis by I. G. Eakins.

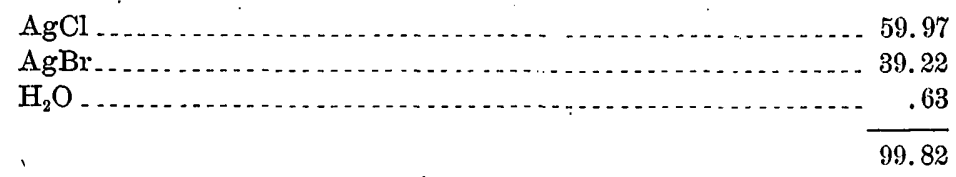

CRYOLITE.

From St. Peters Dome, Pikes Peak district, Colorado: Described by Cross and Hillebrand in Bulletin 20. Massive. Pinkish in color. Specific gravity, 2.972 at $24^{\circ}$. Analysis by W. F. Hillebrand.

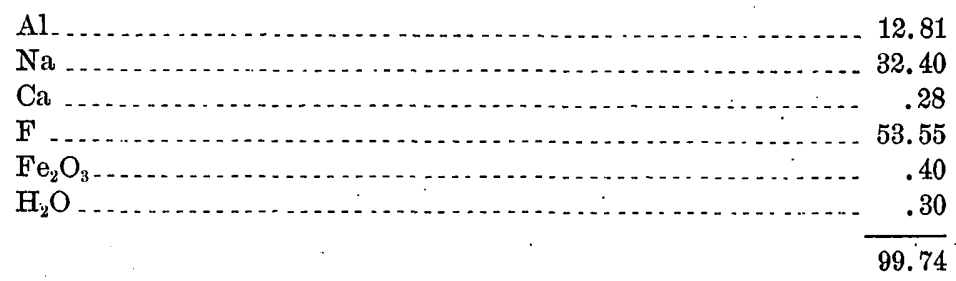

Bull. $220-03-2$ 


\section{PACHNOLITE.}

From St. Peters Dome, Pikes Peak district, Colorado. Described by Cross and Hillebrand in Bulletin 20. Analyses by W. F. Hillebrand.

A. Compact bluish variety. Specific gravity, 2.980 at $22^{\circ}$.

B. Crystalline coating.

C. Fresh, transparent, crystalline material.

D. Carefully selected crystals. Specific gravity, 2.965 at $17^{\circ} ; 2.962$ at $22^{\circ}$.

\begin{tabular}{|c|c|c|c|c|}
\hline & A. & B. & c. & D. \\
\hline $\mathrm{Al}$ & 11.94 & - $12.93,12.92$ & 12.14 & 12.27 \\
\hline $\mathrm{Ca} \ldots$ & 19.32 & $15.27,15.17$. & 18.06 & 18.04 \\
\hline $\mathrm{Mg}$ & .13 & 1.53 & - & - \\
\hline $\mathrm{Na}_{-}$ & 10.43 & 10.28 & 10.23 & 10.25 \\
\hline $\mathrm{K}$ & $\cdots$ & .13 & $\cdots$ & -- \\
\hline $\mathrm{H}_{2} \mathrm{O} \ldots \ldots$ & $7.87,7.95$ & $8.64, \quad 8.7 \dot{9}$ & $8.10,8.11$ & 8.05 \\
\hline \multirow[t]{2}{*}{$\mathrm{F}$} & & & $51.33,51.28$ & « 51.39 \\
\hline & & & 99.86 & 100.00 \\
\hline
\end{tabular}

$a$ By difference.

ELPASOLITE.

From St. Peters Dome, Pikes Peak district, Colorado: Described as a new species by Cross and Hillebrand in Bulletin 20. Incomplete analysis on insufficient material, by W. F. Hillebrand. The fluorine was deduced by calculation on the assumption that the metals are fully combined with it.

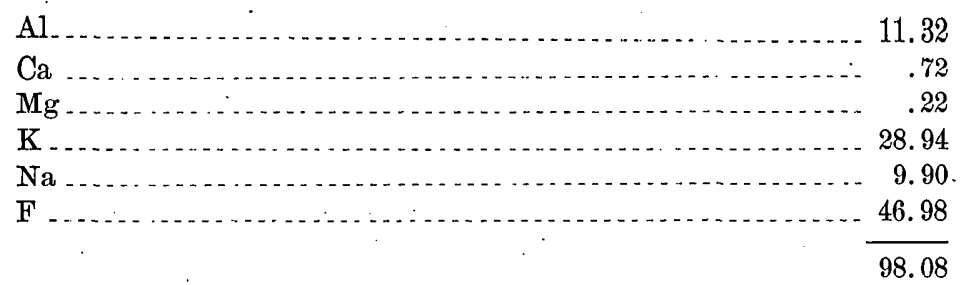

GEARKSUTITE.

From St. Peters Dome, Pikes Peak district, Colorado. Described by Cross and Hillebrand in Bulletin 20. Analysis by W. F. Hillebrand.

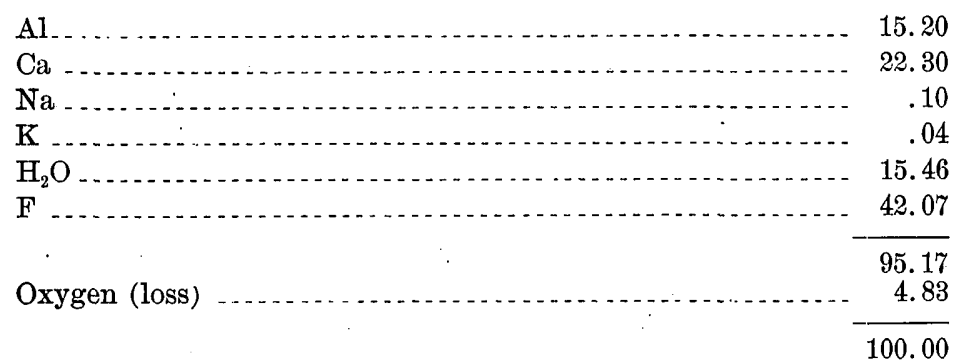


PROSOPITE.

Two samples of prosopite have been analyzed by W. F. Hillebrand, as follows:

A. From St. Peters Dome, Pikes Peak district, Colorado. Described by Cross and Hillebrand in Bulletin 20. Specific gravity, 2.880 at $23^{\circ}$. Mean of four analyses.

B. Pale green, massive variety, from the Dugway mining district, Tooele County, Utah. Specific gravity, 2.87 at $21^{\circ}$. Described by Hillebrand in Bulletin 167.

\begin{tabular}{|c|c|c|}
\hline$\cdot$ & A. & B. \\
\hline $\mathrm{Al} \ldots$ & 22.02 & 20.08 \\
\hline $\mathrm{Ca} \ldots \ldots \ldots$ & 17.28 & 17.55 \\
\hline Mg $\ldots \ldots \ldots$ & .17 & trace \\
\hline $\mathbf{K}$ & $\ldots$. & .12 \\
\hline $\mathrm{Na}$ & .48 & .32 \\
\hline $\mathrm{Cu} . . .$. & $-\ldots$. & .17 \\
\hline F & $\dddot{3} 3.18$ & 28.00 \\
\hline $\mathrm{H}_{2} \mathrm{O} \ldots \ldots$ & 13.46 & 14.24 \\
\hline & 86.59 & 80.48 \\
\hline \multirow[t]{2}{*}{ Oxygen (loss) - } & 13.41 & a 19.52 \\
\hline & 100.00 & 100.00 \\
\hline
\end{tabular}

a Including a little quartz; undetermined.

TYSONITE.

From Cheyenne Mountain, near Pikes Peak, Colorado. Analyzed by W. F. Hillebrand and described by him in Bulletin 167. Corrected specific gravity, 6.14 at $28^{\circ}$.

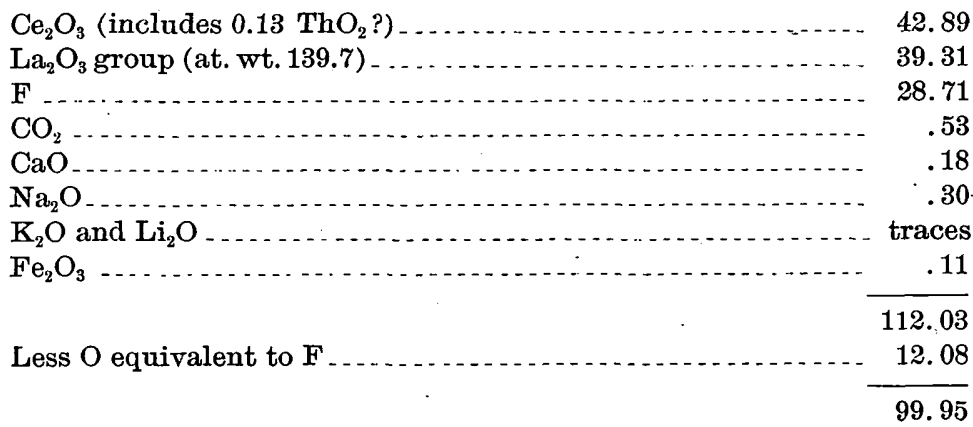




\section{OXIDES AND HYDROXIDES.}

SPINEL.

Variety pleonaste. Separated from a pyroxenite found between South Meadow and Moore creeks, Madison County, Mont. Rock described by Merrill in Proc. U. S. Nat. Mus., vol. 17, p. 659. Specific gravity of spinel, 3.89 at $32^{\circ} .3:$ Analysis by L. G. Eakins.

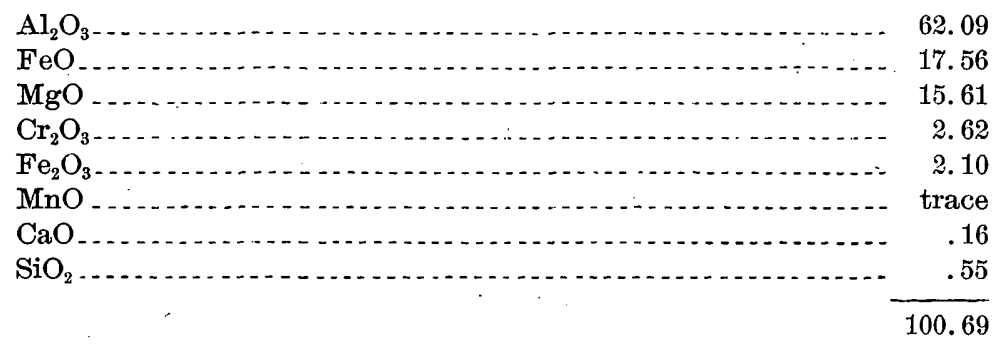

GAHNTTE.

From Gilmore's-ríca mine, Montgomery County, Md., about 1ż iniles-north of Washington, D. C. Color, bottle-green. Specific gravity, 4.59. Analyzed by T. M. Chatard and described by him in Bulletin 9 .

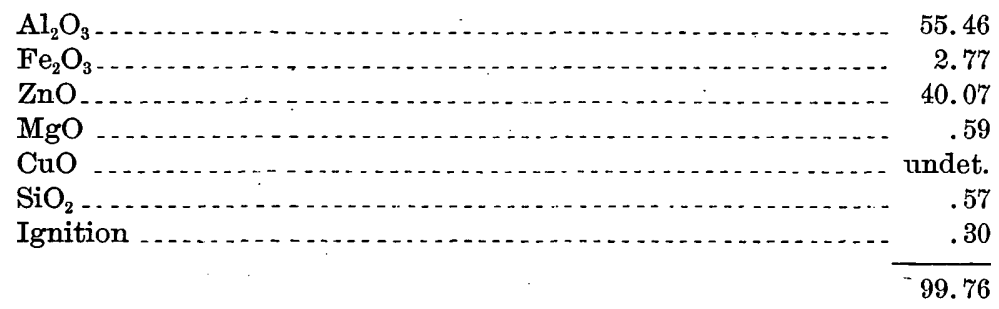

MAGNETITE.

From the Gallatin Range, between Middle and Bozeman creekss, near Bozeman, Mont. Analysis by T. M. Chatard.

Insoluble

0.16

$\mathrm{Fe}_{3} \mathrm{O}_{4}$

96.70

$\mathrm{Al}_{2} \mathrm{O}_{3}$

.04

$\mathrm{MnO}$

$\mathrm{CaO}$

trace

$\mathrm{MgO}$

.07

$\mathrm{TiO}_{2}$

2. 71

$\mathrm{P}_{2} \mathrm{O}$

$\mathrm{S}$

Less $\mathrm{O}$ equivalent to $\mathrm{S}$ .06 
CHROMITE.

From Corundum .Fill, North Carolina. Analysis by 'T. M. Chatard. $\mathrm{Fe}_{2} \mathrm{O}_{3}$ not separately determined.

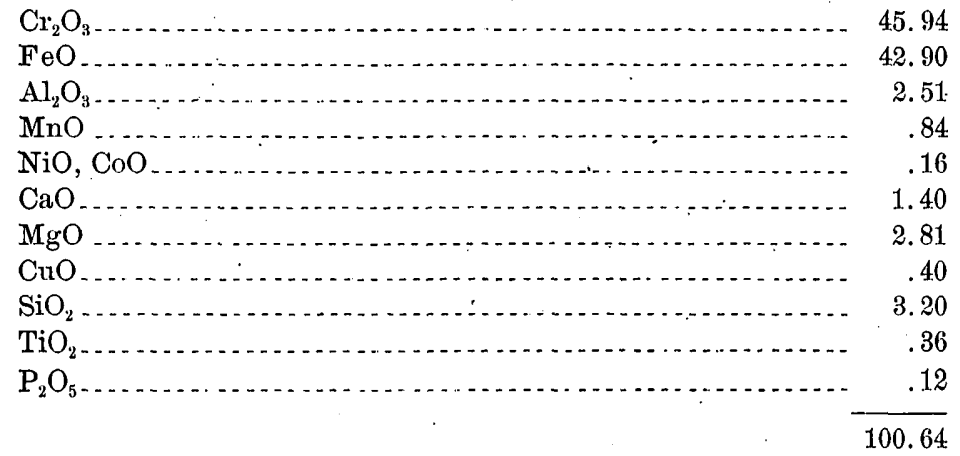

RUTILE.

From near St. Peters Dome, Pikes Peak district, Colorado. Specific gravity, 4.288 at $19^{\circ}$. Analysis by I. G. Eakins.

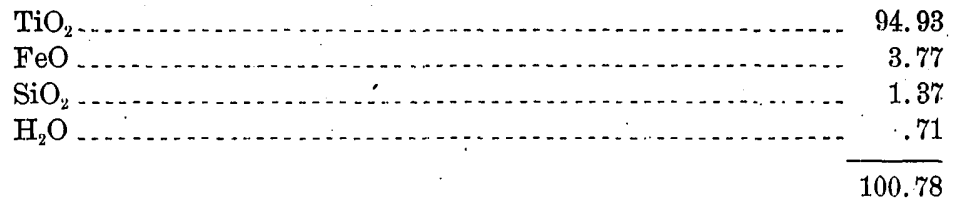

DIASPORE.

From Mount'Robinson, Custer County, Colo. Analysis by L. G. Eakins.

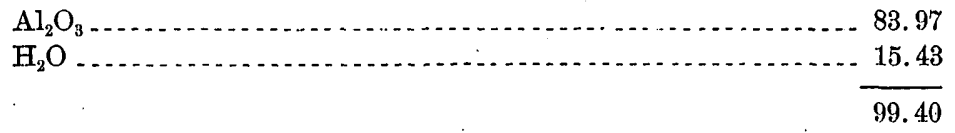

BAUXITE.

Two samples from Jacksonville, Calhoun County, Ala: Alkalies, lime, and magnesia not looked for. Analyses by W. F. Hillebrand.

\begin{tabular}{|c|c|c|}
\hline - & Red. & White. \\
\hline $\mathrm{Al}_{2} \mathrm{O}_{3}$ & 41.00 & 48.92 \\
\hline $\mathrm{Fe}_{2} \mathrm{O}_{3}$ & 25.25 & 2.14 \\
\hline $\mathrm{H}_{2} \mathrm{O}$ at $100^{\circ}$ & .65 & .45 \\
\hline $\mathrm{H}_{2} \mathrm{O}$ by ignition & 20.43 & 23.41 \\
\hline $\mathrm{SiO}_{2}$ & 10.25 & 21.08 \\
\hline $\mathrm{TiO}_{2}$ & 2.53 & 2.52 \\
\hline \multirow{2}{*}{$\mathrm{P}_{2} \mathrm{O}_{5}$} & trace & trace \\
\hline & 100.11 & 98.52 \\
\hline
\end{tabular}


BRUCITE.

From Texas, Lancaster County, Pa. Analysis by E. A. Schneider:

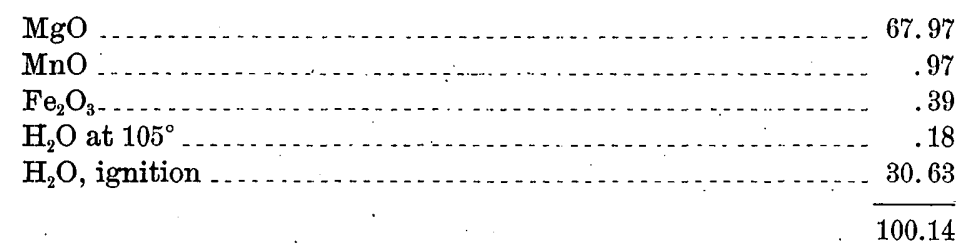

PSILOMELANE.

From a prospect hole on plain south of Round Mountain, Silver Cliff, Colo. Analysis by W. F. Hillebrand. There were strong spectroscopic reactions for strontia and lithia, but these bases were not estimated. A little insoluble gangue is included with the silica.

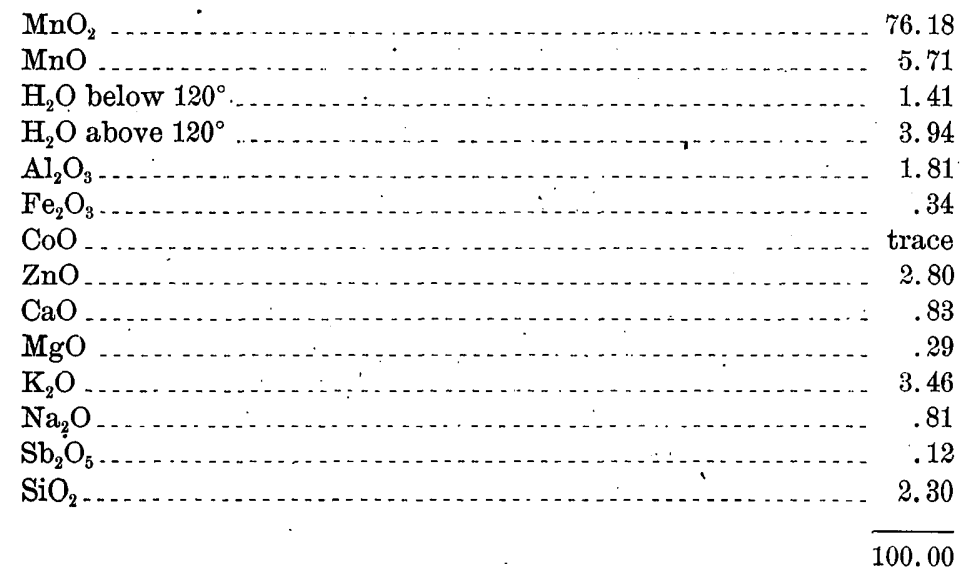




\section{CARBONATES.}

\section{DOLOMITE.}

A. Dolomite 'marble, New York Quarry Company, Tuckahoe, Westchester County, N. Y. Analysis by W. F. Hillebrand.

B. Dolomite marble, Cockeysville, Md. Analysis by J. E. Whitfield.

C. Same as B. Analysis by E. A. Schneider. Another sample.

D. Dolomite marble, Webster, Mass. Analysis by H. N. Stokes.

E. Pink, crystallized dolomite, Joplin, Mo. Analysis by E. T. Allen.

\begin{tabular}{|c|c|c|c|c|c|}
\hline & A. & B. & C. & D. & E. \\
\hline Insoluble_ & 0.62 & ..... & 5.57 & ... & \\
\hline $\mathrm{SiO}_{2 \ldots} \ldots$. &.$\dot{7} 1$ & 0.44 & - & 1.01 & . . . . \\
\hline $\mathrm{Al}_{2} \mathrm{O}_{3}$ &.- & 1.22 & 40 & .17 & $\cdots$ \\
\hline $\mathrm{Fe}_{2} \mathrm{O}_{3} \ldots$ & & & .40 & none & -- \\
\hline $\mathrm{FeO} \ldots$ & $\cdot 21$ & trace & .. & .37 & 0.90 \\
\hline $\mathrm{MnO}$ & $\ldots$ & …... & $\cdots .$. & .08 & .06 \\
\hline $\mathrm{MgO}_{-}$. & 20.71 & 20.87 & 20.30 & 21.35 & 19.65 \\
\hline $\mathrm{CaO} \ldots \ldots$ & 30.68 & 30.73 & 29.08 & 30.82 & 32.05 \\
\hline $\mathrm{CO}_{2}$ & 46.66 & 45.85 & 44.26 & 45.84 & 46.82 \\
\hline $\mathrm{H}_{2} \mathrm{O}_{\ldots} \ldots \ldots$ & .16 & 1.22 & & .09 & .48 \\
\hline $\mathrm{K}_{2} \mathrm{O} \ldots$ & & & & .10 & \\
\hline $\mathrm{Na}_{2} \mathrm{O}_{\ldots} \ldots$ & & & & .01 & $\cdots$ \\
\hline $\mathrm{P}_{2} \mathrm{O}_{5} \ldots \ldots$ & & & & .06 & \\
\hline $\mathrm{TiO}_{2} \ldots$ & & & & trace & \\
\hline \multirow[t]{2}{*}{ S ... } & $\cdots$ & & $\cdots$ & trace & - . \\
\hline & 99.75 & 100.33 & 99.61 & 99.90 & 99.96 \\
\hline
\end{tabular}

SMITHSONITE.

From Marion County, Ark. Bright yellow variety, known locally as "turkey-fat ore." Analysis by H. N. Stokes.

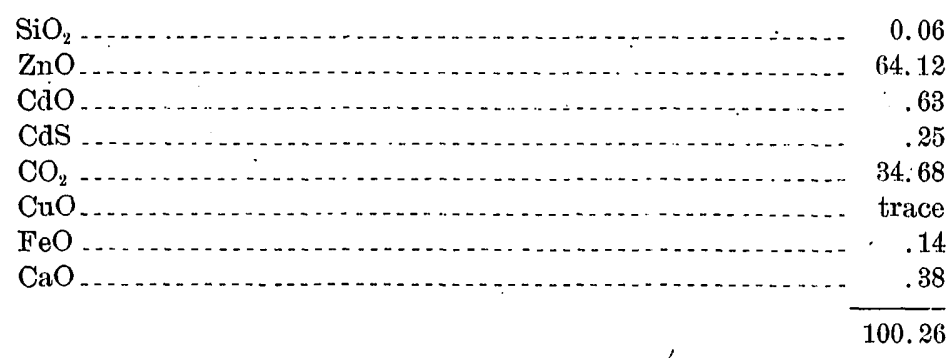




\section{BASTNÄSITE.}

Associated with the tysonite of Cheyenne Mountain, near Pikes Peak, Colorado. Specific gravity, 5.12 at $27^{\circ}$. Analyzed by W. F. Hillebrand and described by him in Bulletin 167.

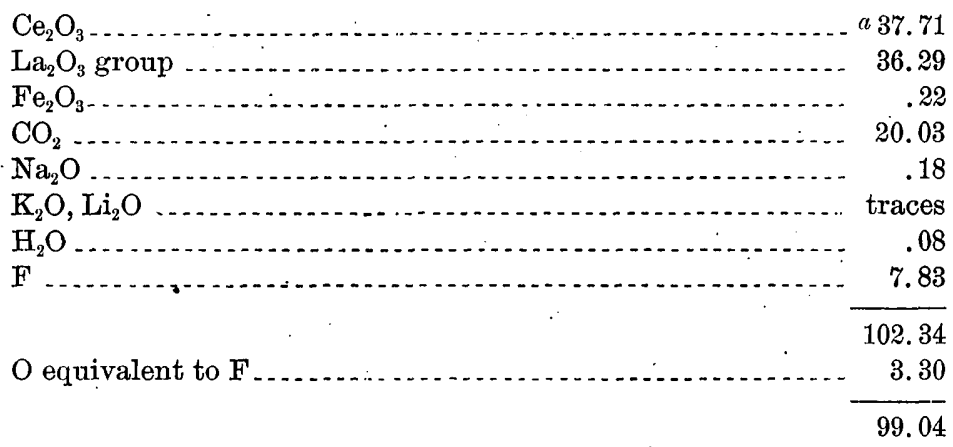

URAO.

Deposited from the waters of Owens Lake, California. Analyses by T. M. Chatard. Described by Chatard in Bulletin 60 . Five sampies analyzed, as follows:

A. The best material. Specific gravity, 2.1473 at $21.7^{\circ}$.

B. Crude urao.

C. Urao deposited upon a grass root.

D. From a small lagoon.

E. From a vat dug on the beach of Owens Lake.

\begin{tabular}{|c|c|c|c|c|c|}
\hline & A. & B. & C. & $\mathrm{D}$. & E. \\
\hline Insoluble, inorganic. . . & \multirow[t]{2}{*}{0.02} & \multirow{2}{*}{$\begin{array}{c}0.22 \\
\end{array}$} & 2.92. & 0.40 & 4.10 \\
\hline Insoluble, organic. & & & .14 & .12 & .27 \\
\hline $\mathrm{SiO}_{2} \ldots \ldots$ & ..... & .10 & .05 & .09 & .04 \\
\hline $\mathrm{Cl} \ldots$ & .19 & 1.57 & 2.73 & .21 & 1.83 \\
\hline $\mathrm{SO}_{3}$ & .70 & .79 & .76 & .63 & .84 \\
\hline $\mathrm{CO}_{2} \ldots \ldots$ & 38.13 & 37.00 & 35.24 & 37.50 & 35.10 \\
\hline $\mathrm{Na}_{2} \mathrm{O} \ldots \ldots \ldots$ & 41.00 & 41.26 & 40.22 & 40.08 & 39.36 \\
\hline $\mathrm{K}_{2} \mathrm{O} \ldots \ldots$ & & & & trace & $\cdots$ \\
\hline \multicolumn{4}{|l|}{$\mathrm{CaO}$.. } & .06 & $\cdots$ \\
\hline \multicolumn{4}{|l|}{$\mathrm{MgO}$. } & .02 & ..... \\
\hline \multirow[t]{2}{*}{$\mathrm{H}_{2} \mathrm{O}$} & 20.07 & 19.62 & 18.31 & 19.94 & 18.58 \\
\hline & 100.11 & 100.56 & $100.37^{\circ}$ & 99.05 & 100.12 \\
\hline \multirow[t]{2}{*}{$\mathrm{O}$ equivalent to $\mathrm{Cl}$} & .04 & .35 & .61 & .05 & .41 \\
\hline & 100.07 & 100.21 & 99.76 & 99.00 & 99.71 \\
\hline
\end{tabular}




\section{SILICATES.}

\section{PETALITE.}

From Peru, Me., associated with spodumene. Analysis by F. W. Clarke.

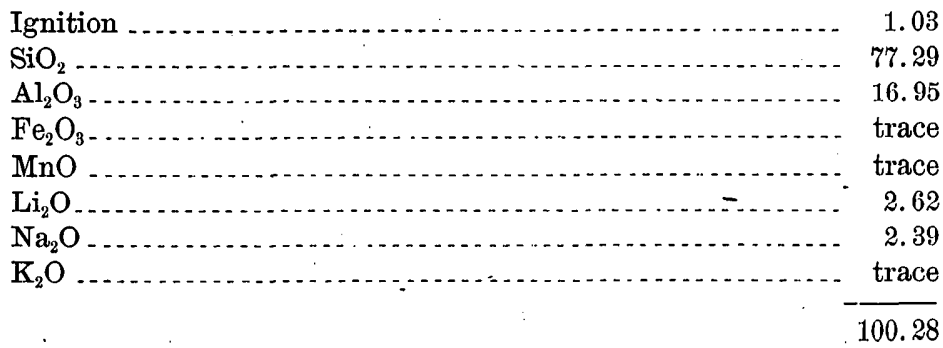

\section{ORTHOCLASE AND MICROCLINE.}

A. Orthoclase from Silver City, Idaho. Occurs as a ganguie mineral. Partial analysis by W. F. Hillebrand.

B. Orthoclase from Mitchell County, N. C. Large cleavage masss. Analysis by E. T. Allen.

C. Feldspar crystal from the nevadite of Chalk Mountain, Summit County, Colo. Analysis by W. F. Hillebrand.

D. Pink orthoclase crystals, from gray porphyry, Johnson Gulch, near Leadville, Colo. Described by Cross in Monograph XII, Appendix A. Analysis by W. F. Hillebrand.

E. Feldspar separated from gabbro. East side of North Fowl Lake, Minnesota. Analysis by W. F. Hillebrand.

F. Feldspar from the elæolite-syenite of Litchfield, Me. Described by Bayley in Bulletin 150, p. 201. Analysis by W. H. Melville.

\begin{tabular}{|c|c|c|c|c|c|c|}
\hline & A. & B. & c. & D. & E. & F. \\
\hline $\mathrm{SiO}_{2}$ & 66.28 & 65.09 & 65.04 & 62.22 & 62.71 & 65.14 \\
\hline $\mathrm{TiO}_{2}$ & $\ldots$ & $\cdots$ & n...... & $\because$ & trace & \\
\hline $\mathrm{Al}_{2} \mathrm{O}_{3}$ & 17.93 & 18.95 & 20.40 & 20.33 & 19.20 & 18. 19 \\
\hline $\mathrm{Fe}_{2} \mathrm{O}_{3}$ & & 36 & & & 1.08 & $\cdots$ \\
\hline $\mathrm{FeO}$. & & .00 & & & .93 & .25 \\
\hline $\mathrm{MnO} \ldots$ & & $\ldots$ & $\ldots$ & $\cdots$ & trace & ... \\
\hline $\mathrm{CaO} \ldots$ & $\cdots$ & .42 & .79 & 2.95 & .44 & .33 \\
\hline $\mathrm{SrO} \ldots$ & & - & 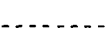 & & trace & \\
\hline $\mathrm{MgO} \ldots$ & $\ldots$ & none & none & $\ldots$ & .81 & .16 \\
\hline $\mathrm{Na}_{2} \mathrm{O}$ & .25 & 2.29 & 4.11 & 3.45 & $2 . \overline{9} 6$ & 1.68 \\
\hline $\mathrm{K}_{2} \mathrm{O}$ & 15.12 & 12.95 & 9.74 & 8.31 & 10.41 & 14.14 \\
\hline $\mathrm{Li}_{2} \mathrm{O} \ldots$ & & & $\operatorname{trace}$ & & none & \\
\hline $\mathrm{H}_{2} \mathrm{O}$ at $100^{\circ} \ldots$ & & & 29 & 100 & .23 & 17 \\
\hline $\mathrm{H}_{2} \mathrm{O}$ above $100^{\circ} \ldots$ & & & . & 1.00 & .92 & .16 \\
\hline & 99.58 & 100.06 & 100.37 & 99.16 & 99.69 & $\cdot 100.06$ \\
\hline
\end{tabular}


G. Flesh-colored microcline from the pegmatite of Jones Falls, Baltimore, Md.

H. Greenish microcline, same locality as $\mathrm{G}$. G and $\mathrm{H}$ described by S. L. Powell in Johns Hopkins Univ. Circular, vol. 12, p. 49. Analyses by W. F. Hillebrand.

I. Anorthoclase from the elæolite-syenite of Peaked Butte, Crazy Mountains, Montana. See Wolff and Tarr in Bull. Mus. Comp. Zoöl., Harvard Coll., vol. 16, No. 12, 1893. Analysis by W. F. Hillebrand.

J. Anorthoclase from red soda-granite, Pigeon Point, Minnesota. Described by Bayley in Bulletin 109.' Analysis by J. E. Whitfield.

K. Anorthoclase from keratophyre, Marblehead Neck, Massachusetts. Described by Sears, Bull. Mus. Comp. Zoöl., Harvard Coll., vol. 16, No. 9, 1893. Analysis by T. M. Chatard.

\begin{tabular}{|c|c|c|c|c|c|}
\hline & G. & H. & I. & $\mathrm{J}$. & $\mathrm{K}$. \\
\hline $\mathrm{SiO}_{2}$ & 65.06 & 68.48 & 62.31 & 65.00 & 65.66 \\
\hline $\mathrm{Al}_{2} \mathrm{O}_{3} \ldots$ & 18.41 & 16.11 & 22.63 & 18.22 & 20.05 \\
\hline $\mathrm{Fe}_{2} \mathrm{O}_{3} \ldots$ & trace & .20 & & 2.64 & trace \\
\hline $\mathrm{FeO}$ & & .17 & & & trace \\
\hline $\mathrm{MnO} \ldots$ & $\therefore-$ & $-\ldots$ & 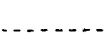 & $\cdots$ & .13 \\
\hline $\mathrm{MgO} \ldots \ldots \ldots$ & .04 & .03 & $\ldots$ & .06 & .18 \\
\hline $\mathrm{CaO}:-$ & .26 & .23 & .63 & 1.06 & .67 \\
\hline $\mathrm{SrO} \ldots \ldots$ & trace & trace & .57 & - & 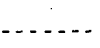 \\
\hline $\mathrm{BaO} \ldots \ldots$ & .13 & .05 & .77 & ..... & $\ldots$ \\
\hline $\mathrm{K}_{2} \mathrm{O} \ldots \ldots$ & 14.30 & 12.99 & 4.79 & 4.18 & 6.56 \\
\hline $\mathrm{Na}_{2} \mathrm{O}$ & 1.60 & 1.27 & 7.68 & 8.40 & 6.98 \\
\hline $\mathrm{Li}_{2} \mathrm{O}$ & trace & trace & & & $\ldots$ \\
\hline $\mathrm{H}_{2} \mathrm{O}$ at $100^{\circ} \ldots$ & .04 & .06 & .16 & & .04 \\
\hline \multirow[t]{2}{*}{$\mathrm{H}_{2} \mathrm{O}$ above $100^{\circ}$} & .26 & .26 & .72 & & .37 \\
\hline & 100.10 & 99.85 & 100.26 & 100.02 & 100.64 \\
\hline
\end{tabular}

ALBITE.

A. From feldspathic schist, central shaft of the Hoosac Tunnel, Berkshire County, Mass. Described by Wolff in Monograph XXIII, pp. 60-187. Analysis by R. B. Riggs.

B, C. From the porphyritic mica-schist of Greylock Mountain, Massachusetts. Described by Wolff, loc. cit. Analyses by R. B. Riggs.

D. From the clrolite-syenite of Litchfield, Me. Described by Bayley in Bulletin 150, p. 201. Specific gravity, 2.622. Analysis by W. H. Melville.

E. From the pegmatite of Jones Falls, Baltimore, Md. Described by S. L. Powell in Johns Hopkins Univ. Circular, vol. 12, p. 49. Analysis by W. F. Hillebrand. 


\begin{tabular}{|c|c|c|c|c|c|}
\hline & A. & B. & c. & D. & E. \\
\hline $\mathrm{SiO}_{2 \ldots} \ldots$ & 69.69 & 68.08 & 67.83 & 68.28 & 63.72 \\
\hline $\mathrm{Al}_{2} \mathrm{O}_{3} \ldots \ldots$ & 18.60 & 20.11 & 19.92 & 19.62 & 22.26 \\
\hline \multicolumn{6}{|l|}{$\mathrm{Fe}_{2} \mathrm{O}_{3} \ldots \ldots$} \\
\hline \multicolumn{6}{|l|}{$\mathrm{FeO}_{-}$} \\
\hline $\mathrm{MgO} \ldots$ & .20 & $(?)$ & (?) & .09 & .08 \\
\hline $\mathrm{CaO} \ldots \ldots . . .$. & trace & trace & trace & $\therefore 31$ & 3.58 \\
\hline \multicolumn{6}{|l|}{$\mathrm{SrO} \ldots$} \\
\hline $\mathrm{Na}_{2} \mathrm{O} \ldots \ldots$ & 10.28 & 11.00 & 11.65 & 10.81 & 8.98 \\
\hline $\mathrm{K}_{2} \mathrm{O} \ldots \ldots$ & .40 & .36 & .25 & .39 & .76 \\
\hline \multicolumn{6}{|l|}{$\mathrm{Li}_{2} \mathrm{O}$} \\
\hline \multicolumn{6}{|l|}{$\mathrm{MnO} \ldots \ldots$} \\
\hline \multicolumn{6}{|l|}{$\mathrm{H}_{2} \mathrm{O}$ below $100^{\circ}$} \\
\hline \multicolumn{6}{|l|}{$\mathrm{H}_{2} \mathrm{O}$ above $100^{\circ} \ldots \ldots$} \\
\hline & 99.59 & 99.86 & 99.77 & 99.82 & 99.88 \\
\hline
\end{tabular}

\section{ANORTHITE.}

A. From Raymond, Me. Associated with idocrase, garnet, pyroxene, and scapolite. Analysis by W. H. Melville.

B. From Phippsburg, Me. Occurrence similar to A. Incomplete analysis by George Steiger.

C. Separated from "hyperite changing to diorite," near Whitaker's ore pit, Wilmington, Del. Described by Chester in Bulletin 59. Analysis by R. B. Riggs.

\begin{tabular}{|c|c|c|c|}
\hline & A. & B. & c. . \\
\hline $\mathrm{SiO}_{2} \ldots$. & 43.13 & 45.62 & 44.09 \\
\hline $\mathrm{Al}_{2} \mathrm{O}_{3}$ & 30.95 & 35.29 & 35.41 \\
\hline $\mathrm{Fe}_{2} \mathrm{O}_{3}$ & 1.04 & $-E_{0}$ & .51 \\
\hline $\mathrm{FeO} \ldots . . . .$. & trace & , & $\ldots$. \\
\hline $\mathrm{MnO} \ldots \ldots \ldots$ & trace & - ...... & - n \\
\hline $\mathrm{CaO}$ & 19.71 & 17.31 & 18.47 \\
\hline MgO $\ldots \ldots$ & .31 & 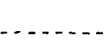 & none \\
\hline $\mathrm{K}_{2} \mathrm{O}$ & 1.29 & & .19 \\
\hline $\mathrm{Na}_{2} \mathrm{O} \ldots \ldots$ & .69 & & .99 \\
\hline $\mathrm{Li}_{2} \mathrm{O}$ & trace & & \\
\hline $\mathrm{H}_{2} \mathrm{O}$ at $100^{\circ} \ldots \ldots$ & .22 & & 35 \\
\hline \multirow[t]{2}{*}{$\mathrm{H}_{2} \mathrm{O}$ above $100^{\circ}$. } & 2.80 & & .50 \\
\hline & 100.14 & 98.22 & 100.01 \\
\hline
\end{tabular}




\section{SODA-LIME FELDSPARS.}

A. Transparent oligoclase, from Bakersville, N. C. Analysis by F. W. Clarke.

B. Feldspar separated from porphyrite; Sugar Loaf, northwest of Elk Mountain, Tenmile district, Summit County, Colo. Analysis by W. F. Hillebrand. Much altered.

C. Feldspar separated from andesite; mesa northwest of Parkdale, Colo. Analysis by W. F. Hillebrand.

D. Feldspar separated from gabbro; Brandywine Creek, Wilmington, Del. Specific gravity, 2.592-2.877. Described by Chester in Bulletin 59. Analysis by R. B. Riggs.

E. Feldspar separated from the gabbro of Ashland County, Wis. Analysis by W. F. Hillebrand.

F. Feldspar separated from diabase; near SE. corner of sec. 13, T. 47. N., R. 46 W., Michigan. Described by Van Hise in Monograph XIX. Analysis by T. M. Chatard.

\begin{tabular}{|c|c|c|c|c|c|c|}
\hline & A. & B. & C. & D. & E. & $\mathrm{F}$. \\
\hline $\mathrm{SiO}_{2-}$ & 62.92 & 62.96 & 63.02 & 70.37 & 53.30 & 51.18 \\
\hline $\mathrm{TiO}_{2} \ldots$ & 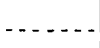 & - & |... & - & trace & ... \\
\hline $\mathrm{Al}_{2} \mathrm{O}_{3}$ & 25.32 & 21.51 & 23.05 & 18.36 & 29.03 & 27.00 \\
\hline $\mathrm{Fe}_{2} \mathrm{O}_{3}$ & trace & & & .58 & .55 & 3.19 \\
\hline $\mathrm{FeO}$ & . . . . . & .32 & & undet. &.$\tilde{23}$ & undet. \\
\hline $\mathrm{CaO}$ & 4.03 & 4.00 & 3.39 & 5.08 & 11.40 & 11.70 \\
\hline $\mathrm{SrO}$. & & .13 & trace & & trace & \\
\hline $\mathrm{BaO}$ & & $\ldots$. & .... & $\ldots$ & trace & $\cdots$ \\
\hline $\mathrm{MgO}$. & & .30 & $\operatorname{trace}$ & .04 & .13 & 1.92 \\
\hline $\mathrm{MnO}$ & trace & $\ldots$ & -. & trace & none & .17 \\
\hline $\mathrm{K}_{2} \mathrm{O} \ldots$ & .96 & 1.60 & 3.92 & .63 & .40 & .41 \\
\hline $\mathrm{Na}_{2} \mathrm{O}$ & 6.18 & 6.15 & 6.76 & 4.32 & 4.87 & 3.48 \\
\hline $\mathrm{Li}_{2} \mathrm{O} \ldots$ & - & trace & non $\theta^{\circ}$ & & none & \\
\hline $\mathrm{P}_{2} \mathrm{O}_{5}$ & & & & & trace & \\
\hline $\mathrm{H}_{2} \mathrm{O} \ldots$ & .25 & 2.78 & .26 & .45 & .23 & 1.19 \\
\hline & 99.66 & 99.75 & 100.40 & 99.83 & 100.14 & 100.24 \\
\hline
\end{tabular}

G, H. Feldspars separated from olivine-diabase; NE. $\frac{1}{4}$ sec. $13, \mathrm{~T}$. 45 N., R, 1 W., Wisconsin. See Van Hise, Monograph XIX. Analyses by T. M. Chatard.

I. Feldspar from gabbro; southern half of sec. 14, T. 44 N., R. 4 W., Wisconsin. See Van Hise, loc. cit. Analysis by T. M. Chatard.

The following feldspars were separated from Minnesota gabbros for W. S. Bayley and analyzed by W. F. Hillebrand: 
J. From average gabbro, south quarter post, sec. 35, T. 61 N., R. $12 \mathrm{~W}$.

K. From gabbro, NW. $\frac{1}{4}$ of SE. $\frac{1}{4}$ sec. 23, T. 62 N., R. 10.

L. From gabbro, center of sec. 25, T. 64 N., R. 8 .

M. From gabbro, Duluth and Iron Range Railroad.

N. O. Two feldspars separated from the amphibolite of Palmer Center, Mass. Analyses by W. F. Hillebrand. Specific gravity of $\mathrm{N}, 2.667$ at $24^{\circ}$; of $\mathrm{O}, 2.677$ at $22^{\circ}$. In $\mathrm{N}$, calculation gives about 7.4 and in $\mathrm{O} 7.6$ per cent of admixed quartz.

\begin{tabular}{|c|c|c|c|c|c|}
\hline & G. & H. & I. & J. & $\mathbf{K}$. \\
\hline $\mathrm{SiO}_{2} \ldots$ & 61.65 & 56.15 & 51.99 & 51.89 & 52.50 \\
\hline $\mathrm{Al}_{2} \mathrm{O}_{3} \ldots \ldots$ & 19.91 & 26.05 & 29.32 & 29.68 & 30.15 \\
\hline $\mathrm{Fe}_{2} \mathrm{O}_{3}$ & 2.28 & 1.98 & 123 & .32 & .47 \\
\hline $\mathrm{FeO}$ & undet. & undet. & & .37 & .15 \\
\hline $\mathrm{MnO} \ldots \ldots$ & $\operatorname{trac} \theta$ & .13 & trace & |... & . \\
\hline $\mathrm{CaO}$ & $4: 12$ & $8.70^{\circ}$ & 12.60 & 12.62 & 12.82 \\
\hline $\mathrm{MgO}$ & .61 & .54 & .63 & .38 & .10 \\
\hline $\mathrm{Na}_{2} \mathrm{O}$ & 4.74 & 4.79 & 2.91 & 3.87 & 3.72 \\
\hline $\mathrm{K}_{2} \mathrm{O} \ldots$ & 5.72 & 1.56 & .28 & .50 & .53 \\
\hline \multirow{4}{*}{$\begin{array}{l}\mathrm{H}_{2} \mathrm{O} \text { at } 100^{\circ} \\
\mathrm{H}_{2} \mathrm{O} \text { above } 100^{\circ} \\
\mathrm{TiO}_{2}\end{array}$} & 95 & .13 & .03 & .07 & 25 \\
\hline & & .64 & .54 & .39 & \\
\hline & & & & & $\operatorname{trac} \theta$ \\
\hline & 99.98 & 100.67 & 99.53 & 100.09 & 100.69 \\
\hline \multicolumn{2}{|c|}{. } & L. & M. & $\mathrm{N}$. & 0. \\
\hline \multirow{5}{*}{\multicolumn{2}{|c|}{$\begin{array}{l}\mathrm{SiO}_{2} 2 \\
\mathrm{Al}_{2} \mathrm{O}_{3} \\
\mathrm{Fe}_{2} \mathrm{O}_{3} \\
\mathrm{FeO} \\
\mathrm{CaO}\end{array}$}} & 52.61 & 53.45 & 62.91 & 60.90 \\
\hline & & 29.80 & 29.77 & 23.37 & 24.97 \\
\hline & & .57 & .33 & $\operatorname{trac} \theta$ & $\operatorname{trace}$ \\
\hline & & .23 & .15 & ...... & $\ldots$ \\
\hline & & 12.25 & 11.33 & 5.83 & 7.85 \\
\hline \multicolumn{2}{|c|}{$\mathrm{MgO}$} & .20 & .11 & $\ldots$ & $\cdots$ \\
\hline \multirow{2}{*}{\multicolumn{2}{|c|}{$\begin{array}{l}\mathrm{Na}_{2} \mathrm{O} \\
\mathrm{K}_{2} \mathrm{O}\end{array}$}} & 3.80 & 4.33 & 7.78 & 6.26 \\
\hline & & $: 53$ & .68 & .20 & . .16 \\
\hline \multirow{2}{*}{\multicolumn{2}{|c|}{$\begin{array}{l}\mathrm{H}_{2} \mathrm{O} \text { at } 100^{\circ} \\
\mathrm{H}_{2} \mathrm{O} \text { above } 100^{\circ}\end{array}$}} & .29 & .23 & .42 & .48 \\
\hline & & & & & \\
\hline \multicolumn{2}{|c|}{$\mathrm{TiO}_{2}$} & trace & trace & trace & trace \\
\hline \multirow{2}{*}{\multicolumn{2}{|c|}{$\mathrm{MnO} \ldots$}} & trace & & & $\therefore-\ldots$. \\
\hline & & 100.28 & 100.38 & 100.51 & 100.62 \\
\hline
\end{tabular}


LEUCITE.

From Mount Vesuvius. A fine crystal. Analysis by George Steiger.

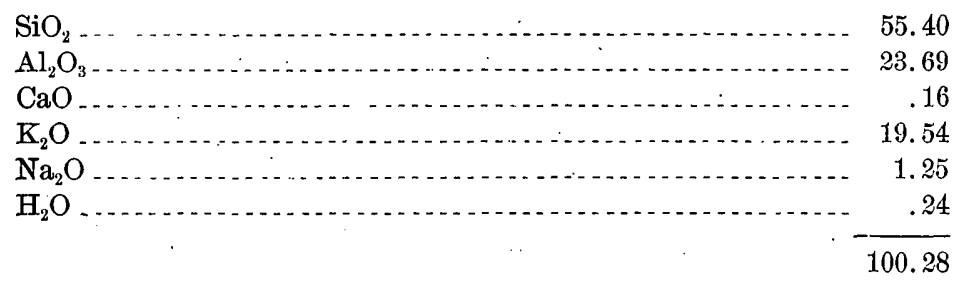

ENSTATITE.

A. Enstatite from Granville, Mass. Slightly altered. Described by Emerson in Monograph XXIX. Analysis by W. F. Hillebrand.

B. Enstatite separated from the San Emigdio meteorite, found in San Bernardino County, Cal. Analysis by J. E. Whitfield.

C. White, fibrous mineral, near enstatite, from seams in chrysolite rock, Corundum Hill, North Carolina.

D. Altered enstatite, Corundum Hill. Analyses C and D by T. M. Chatard. (See Bulletin 42.) Specific gravity, 2.872.

E. Bronzite separated from the websterite of Hebbville, Md. Described by Williams in Am. Geologist, vol. 6, p. 35. Analysis by T. M. Chatard.

\begin{tabular}{|c|c|c|c|c|c|}
\hline & A. & B. & C. & $\mathrm{D}$. & E. \\
\hline $\mathrm{SiO}_{2-}$ & 54.04 & 54.42 & 56.39 & 56.58 & 54.53 \\
\hline $\mathrm{TiO}_{2}$ & none & - & none & none & undet. \\
\hline $\mathrm{Al}_{2} \mathrm{O}_{3}$ & .52 & - n.... & 2.31 & 1.74 & 1.93 \\
\hline $\mathrm{Fe}_{2} \mathrm{O}_{3}$ & 1.51 & $\ldots$ & .16 & 1.89 & 1.70 \\
\hline $\mathrm{FeO} \ldots$ & 3.90 & 14.03 & 1.96 & 3.67 & 8.92 \\
\hline $\mathrm{MnO}$ & .11 & & & .21 & .28 \\
\hline $\mathrm{NiO} \ldots . . . .$. & .23 & & & $\ldots$ & $-\cdots$ \\
\hline $\mathrm{Cr}_{2} \mathrm{O}_{3}$ & .14 & & & .24 & .30 \\
\hline $\mathrm{MgO}_{.}$. & 34.40 & 29.11 & 34.57 & 30.34 & 29.51 \\
\hline $\mathrm{CaO} \ldots \ldots$ & none & 2.46 & .04 & .59 & 2.25 \\
\hline Alkalies & .08 & - & - & .17 & $\ldots$ \\
\hline $\mathrm{P}_{2} \mathrm{O}_{5}$ & none & & & & trace \\
\hline $\mathrm{CO}_{2} \ldots \ldots$ & 1.32 & & & & .... \\
\hline $\mathrm{H}_{2} \mathrm{O}$ at $100^{\circ} \ldots \ldots$ & .70 & & 4.32 & 4.55 & 1,14 \\
\hline \multirow[t]{2}{*}{$\mathrm{H}_{2} \mathrm{O}$ above $100^{\circ} \ldots \ldots$} & 3.07 & & צ. & \pm .00 & 1.14 \\
\hline & 100.02 & 100.02 & 99.75 & 99.98 & 100.56 \\
\hline
\end{tabular}




\section{HYPERSTHENE.}

A. From the basalt of Mount Thielsen, Oregon. Incomplete analysis by T. M. Chatard.

B. From gabbro, SE. $\frac{1}{4}$ sec. 20 , T. 65 N., R. 4 W., Minnesota. Described by Bayley in Jour. Geol., vol. 3, p. 1. Analysis by E. A. Schneider.

C. From the augite-andesite of the Tokajer-Berg, Hungary. Analysis by W. F. Hillebrand. Specific gravity, 3.495 at $25^{\circ}$.

D, E, F. Three specimens separated from the hypersthene-andesite of the Buffalo Peaks, Colorado. Deseribed by Cross in. Bulletin 1. Analyses by W. F. Hillebrand. In D and $\mathrm{E}$ alkalies were disregarded. In $\mathrm{E}$ and $\mathrm{F}$ all the iron is given as $\mathrm{FeO}$. Specific gravity of $\mathrm{F}, 3.307$ at $23^{\circ}$.

\begin{tabular}{|c|c|c|c|c|c|c|}
\hline 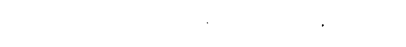 & A. & B. & c. & D. & E. & F. \\
\hline $\mathrm{SiO}_{2-}$ & 53.31 & 48.44 & 51.44 & 51.70 & 51.16 & 50.04 \\
\hline $\mathrm{TiO}_{2} \ldots$ & 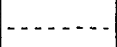 & undet. & .73 & . & 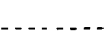 & (-..... \\
\hline $\mathrm{Al}_{2} \mathrm{O}_{3} \ldots \ldots$ & 5.99 & 7.91 & .60 & 1.72 & 2.15 & 2.91 \\
\hline $\mathrm{Fe}_{2} \mathrm{O}_{3}$ & & .33 & 2.28 & .30 & & \\
\hline $\mathrm{FeO} \ldots$ & 13.43 & 20.88 & 20.77 & 18.00 & 18.36 & 17.81 \\
\hline $\mathrm{MnO}$ & ....... & .92 & .88 & .36 & .36 & .12 \\
\hline $\mathrm{MgO}$ & 21.69 & 19.35 & 19.93 & 25.09 & 24.25 & 21.74 \\
\hline $\mathrm{CaO} \ldots \ldots$ & 3.69 & 1.44 & 3.80 & 2.87 & 3.81 & 6.70 \\
\hline $\mathrm{Na}_{12} \mathrm{O} \ldots \ldots$ & . & $\cdots$ & . & 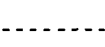 & 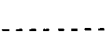 & .27 \\
\hline $\mathrm{H}_{2} \mathrm{O}$ at $100^{\circ}$ & & .08 & & & & \\
\hline \multirow[t]{2}{*}{$\mathrm{H}_{2} \mathrm{O}$ above $100^{\circ}$} & $\ldots \ldots \ldots$ & none & & 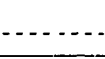 & & $\ldots$ \\
\hline & 98.11 & 99.35 & 100.43 & 100.04 & 100.09 & 99.59 \\
\hline
\end{tabular}




\section{PYROXENES.}

A. Diopside separated from the websterite of Hebbville, Md. Described by G. H. Williams, Am. Geologist, vol. 6, p. 35. Analysis by T. M. Chatard.

B. Diopside from the leucite rocks of the Leucite Hills, Wyoming. Described by Cross, Am. Jour. Sci., 4th ser., vol. 4, p. 115. Specific gravity, 3.290 at $20^{\circ}$. Analysis by W. F. Hillebrand.

C. Pyroxene from Moriah, N. Y. The source of associated serpentine. Analysis by Charles Catlett.

D. Dark-gray pyroxene, Montville, N. J. The source of associated serpentine. Analysis by Charles Catlett.

E. Diallage from the gabbro of Ashland County, Wis. Analysis by W. F. Hillebrand.

F. Jeffersonite from Franklin Furnace, N. J. Analyzed by W. F. Hillebrand and described by him in Bulletin 167.

\begin{tabular}{|c|c|c|c|c|c|c|}
\hline & A. & B. & c. & $\mathrm{D}$. & $\mathrm{E}:$ & F. \\
\hline $\mathrm{SiO}_{2}$ & 51.80 & 50.86 & 55.36 & 51.45 & 49.80 & 51.70 \\
\hline $\mathrm{TiO}_{2} \ldots \ldots$ & .13 & 3.03 & $\cdots$ & $\ldots$ & 1. 29 & $\ldots$ \\
\hline $\mathrm{Al}_{2} \mathrm{O}_{3} \ldots$ & 2.21 & $\cdots$ & .22 & 2.94 & 2.86 & .36 \\
\hline $\mathrm{Cr}_{2} \mathrm{O}_{3} \ldots$ & .51 & $\ldots$ & none & & $\therefore$ & .... \\
\hline $\mathrm{Fe}_{2} \mathrm{O}_{3} \ldots$ & 1.29 & 1.19 & .18 & 1.06 & 2.48 & .37 \\
\hline $\mathrm{FeO} \ldots \ldots$ & 3.50 & 1.82 & .57 & .96 & 10.82 & $\ldots$ \\
\hline $\mathrm{MnO} .$. & trace & .03 & trace & trace & .37 & 7.43 \\
\hline $\mathrm{ZnO}$ & - & $\ldots$ & $\ldots$ & - & $\ldots$ & 3.31 \\
\hline $\mathrm{MgO}$. - & 17.76 & 17.42 & 19.53 & 18.43 & 15.33 & 12.57 \\
\hline $\mathrm{CaO}$ & 20.99 & 23.32 & 24.48 & 24.02 & 16.50 & 23.68 \\
\hline $\mathrm{Na}_{2} \mathrm{O}$ & undet. & .76 & & & .51 & .12 \\
\hline $\mathrm{K}_{2} \mathrm{O}_{\ldots} \ldots$ & undet. & .42 & & &. .12 & trace \\
\hline $\mathrm{P}_{2} \mathrm{O}_{5}$ & trace & & & & trace & $\therefore-$ \\
\hline \multirow[t]{2}{*}{$\mathrm{H}_{2} \mathrm{O}$} & .65 & .31 & & 1.08 & .33 & .65 \\
\hline & 98.84 & 99.16 & 100.34 & 99.94 & 100.41 & 100.19 \\
\hline
\end{tabular}


G. Augite from nepheline-basalt, Black Mountain, Uvalde quadrangle, Texas. Violet colored. Analysis by W. F. Hillebrand:

H. Augite from dolerite dike, near Valmont, Colo. See Cross, Monograph XXVII. Analysis by L. G. Eakins.

I. Augite from tinguaite, Two Buttes, Colo. Specific gravity, 3.43 at $28^{\circ}$. Analysis by W. F. Hillebrand.

J. Pyroxene from syenitic lamprophyre, Two Buttes, Colo. Specific gravity, 3.45 at $25^{\circ}$. Analysis by W. F. Hillebrand.

K. Augite from granite, north end of Blue Mountains, Silver Cliff, Colo. Specific gravity, 3.225 at $18^{\circ}$. Analysis by L. G. Eakins.

L. Augite from the Golden King dike, Silver Cliff, Colo. Specific gravity, 3.281 at $13^{\circ}$. Analysis by L. G. Eakins.

M. Pyroxene from norite, dike east of Sugar Loaf, Boulder County, Colo. Analysis by I. G. Eakins.

\begin{tabular}{|c|c|c|c|c|c|c|c|}
\hline & G. & H. & I. & J. & $\mathbf{K}$. & L. & M. \\
\hline $\mathrm{SiO}_{2}-$ & 45.23 & 49.10 & 47.54 & 51.27 & $\cdot 48.72$ & 54.87 & 47.32 \\
\hline $\mathrm{TiO}_{2} \ldots$. & 4.28 & - & 3.00 & .70 & & & - - \\
\hline $\mathrm{ZrO}_{2} \ldots$ & $\ldots$ & . & none & none & $\ldots$ & -..- & $\ldots$ \\
\hline $\mathrm{Al}_{2} \mathrm{O}_{3}$ & 7.73 & 7.95 & 4.14 & 3.05 & 9.27 & 6.34 & 6.37 \\
\hline $\mathrm{Cr}_{2} \mathrm{O}_{3} \ldots$ & - & & trace? & none & . & $\cdots$ & 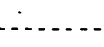 \\
\hline $\mathrm{Fe}_{2} \mathrm{O}_{3}$ & 2.95 & $\cdots$ & 5.64 & 3.08 & 3.77 & 2.88 & 2.56 \\
\hline $\mathrm{FeO}$. & 4.07 & 8.30 & 6.42 & 4.34 & 6.34 & 4.61 & 14.40 \\
\hline $\mathrm{MnO}$. & .07 & $\ldots$ & .36 & .28 & .34 & .14 & (n... \\
\hline $\mathrm{MgO}$ & 12.25 & 12.37 & 10.05 & 14. 21 & 14.67 & 14.47 & 13.43 \\
\hline $\mathrm{CaO}$ & 23.37 & 22.54 & 21.57 & 22.58 & 16.79 & 15.87 & 16.08 \\
\hline $\mathrm{Na}_{22} \mathrm{O}$ & .47 & trace & 1.38 & .67 & .19 & .28 & \\
\hline $\mathrm{K}_{2} \mathrm{O} \ldots \ldots$ & .12 & trace & .12 & .06 & & & \\
\hline $\mathrm{Li}_{2} \mathrm{O} \ldots$ & trace & & trace & & & & \\
\hline $\mathrm{BaO} \ldots$ & none & & none & none & & & \\
\hline $\mathrm{SrO} \ldots$ & none & & none & none & & & \\
\hline $\mathrm{NiO}$ & .05 & & trace & .03 & & & \\
\hline $\mathrm{P}_{2} \mathrm{O}_{5}$ & none & & & & & & \\
\hline \multirow[t]{2}{*}{$\mathrm{H}_{2} \mathrm{O}_{-}$} & .37 & $\therefore$ & undet. & undet. & .18 & .31 & \\
\hline & 100.96 & 100.26 & 100.22 & 100.27 & 100.27 & 99.77 & 100.16 \\
\hline
\end{tabular}

Bull. 220-03-3 
N. Pyroxene from Italian Mountain, Gunnison County, Colo. Associated with idocrase, scapolite, garnet, epidote, etc. Analysis by L. G. Eakins. Specific gravity, 3.312 at $16.7^{\circ}$.

O. Augite from basalt, 6 miles northeast of Grants, Mount Taylor region, New Mexico. Analysis by T. M. Chatard.

P. Pyroxene from peridotite, east of Fort Ellis, Mont. Partial analysis by $\mathrm{F}$. W. Clarke.

Q. Pyroxene from basalt, east side of Bozeman Creek, $2 \frac{1}{2}$ miles southeast of Bozeman, Mont. Described by Merrill, Proc. U. S. Nat. Mus., vol. 17, p. 637 . Analysis by L. G. Eakins.

\begin{tabular}{|c|c|c|c|c|}
\hline & N. & 0. & P. . & Q. \\
\hline $\mathrm{SiO}_{2--}$ & 47.53 & 47.06 & 51.95 & 52.50 \\
\hline $\mathrm{TiO}_{2} \ldots \ldots$ & ...... & $1.82^{\circ}$ & $\cdots$ & $\ldots$ \\
\hline $\mathrm{Al}_{2} \mathrm{O}_{3} \ldots \ldots \ldots$ & 9.88 & 7.77 & 4.21 & 2.26 \\
\hline $\mathrm{Cr}_{2} \mathrm{O}_{3} \ldots \ldots$ & $\ldots . .$. & trace & $\ldots .$. & 1.07 \\
\hline $\mathrm{Fe}_{2} \mathrm{O}_{3}$ & 1.79 & 1.30 & ........ & 2.05 \\
\hline $\mathrm{FeO}_{\ldots} \ldots \ldots \ldots$ & .91 & 8.15 & 5.90 & 2.47 \\
\hline $\mathrm{MgO} \ldots \ldots \ldots$ & trace & .20 & undet. & trace \\
\hline $\mathrm{NiO}, \mathrm{CoO} \ldots \ldots . .$. & $\ldots$. & trace & - . . . & ..... \\
\hline $\mathrm{MgO} \ldots \ldots \ldots$ & 14.43 & 13.52 & 13.81 & 17.11 \\
\hline $\mathrm{CaO} \ldots$ & -25.46 & 19.33 & 23.32 & 21.70 \\
\hline $\mathrm{Na}_{2} \mathrm{O}_{-}$. & trace & .33 & & .35 \\
\hline $\mathrm{K}_{2} \mathrm{O} \ldots$ & . & .11 & & .07 \\
\hline $\mathrm{P}_{2} \mathrm{O}_{5}$ & $\cdots \ldots$ & .06 & & ... \\
\hline \multirow[t]{2}{*}{$\mathrm{H}_{2} \mathrm{O} \ldots \ldots$} & .30 & .20 & undet. & .64 \\
\hline & 100.30 & 99.85 & 99.19 & 100.22 \\
\hline
\end{tabular}

AEGIRITE.

From Magnet Cove, Arkansas. Analysis by George Steiger.

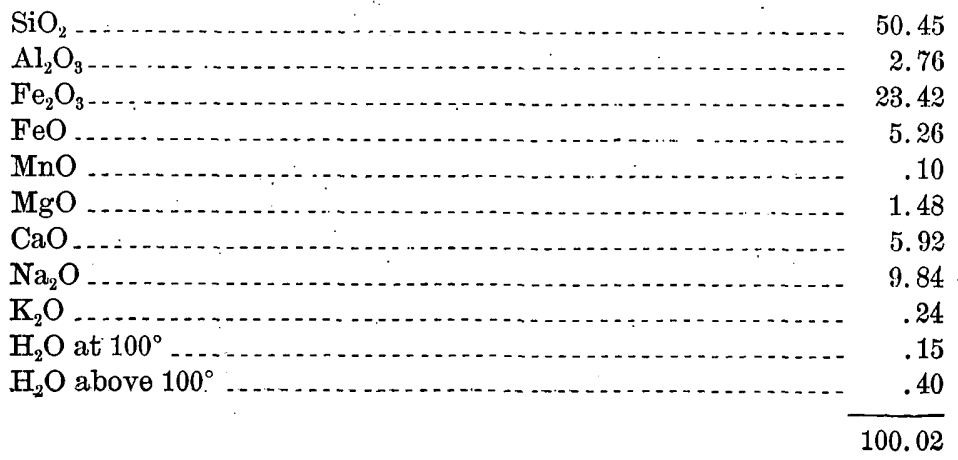




\section{JADEITE.}

The following analyses of jadeite, all by F. W: Clarke, were discussed by Clarke and Merrill in the Proceedings of the United States National Museum for 1888. They all represent worked material.

A. Light-colored bead, mottled with emerald green, from State of Oaxaca, Mexico. Specific gravity, 3.007, determined by William Hallock.

B. Carved head, light green, from Zaachita, Oaxaca. Specific gravity, 3.190, Hallock.

C. Fragment from Sardinal, Costa Rica; pale green, translucent. Specific gravity, 3.32, Clarke.

D. Fragment from Culebra, Costa Rica; light green, granular, opaque; quite impure. Specific gravity, 3.27, Clarke.

\begin{tabular}{|c|c|c|c|c|}
\hline & A. & B. & C. & D. \\
\hline $\mathrm{SiO}_{2}$ & 58.88 & 58.18 & 59.18 & 58.33 \\
\hline $\mathrm{Al}_{2} \mathrm{O}_{3}$ & 25.93 & 23.53 & 22.96 & 21.63 \\
\hline $\mathrm{Cr}_{2} \mathrm{O}_{3}$ & .12 & $\ldots$ & 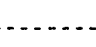 & $\therefore \ldots$ \\
\hline $\mathrm{Fe}_{2} \mathrm{O}_{3} \ldots \ldots$ & & & & 1.71 \\
\hline $\mathrm{FeO} \ldots \ldots . . . . .$. & .24 & 1.67 & 1.87 & .73 \\
\hline $\mathrm{MgO} \ldots$ & .36 & 1.72 & .67 & 3.09 \\
\hline $\mathrm{CaO} . .$. & .40 & 2.35 & 1.52 & 4.92 \\
\hline $\mathrm{Na}_{2} \mathrm{O}$ & 11.64 & 11.81 & 12.71 & 8.13 \\
\hline $\mathrm{K}_{2} \mathrm{O}$ & .63 & .77 & trace & .22 \\
\hline \multirow[t]{2}{*}{ Ignition } & 1.81 & .53 & .90 & .93 \\
\hline & 100.01 & 100.56 & 99.81 & 99.69 \\
\hline
\end{tabular}


WOLLASTONITE.

Compact variety, from Diana, N. Y. Partial analysis by E. A. Schneider:

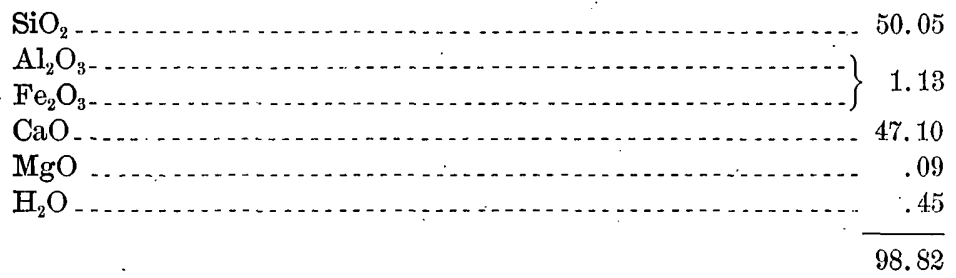

PECTOLITE.

A. Stone hammer, at first supposed to be jade, collected among the Eskimo of Point Barrow, Alaska. Analyzed by F. W. Clarke, and described by him in Bulletin 9. Pale apple-green, tough, compact, highly polished. Specific gravity, 2.873.

B. Radiated pectolite from Bergen Hill, New Jersey. Analyzed by E. A. Schneider.

C. Añother sample from Bergen Hill. Analysis by George Steiger.

\begin{tabular}{|c|c|c|c|}
\hline & A. & B. & C. \\
\hline $\mathrm{SiO}_{2} \ldots \ldots$ & 53.94 & 53.11 & 53.34 \\
\hline $\mathrm{Al}_{2} \mathrm{O}_{3}+\mathrm{Fe}_{2} \mathrm{O}_{3} \ldots \ldots$ & .58 & .40 & .33 \\
\hline $\mathrm{MnO}$ & ..... & .81 & .45 \\
\hline $\mathrm{CaO} \ldots . .$. & 32.21 & 33.88 & 33.23 \\
\hline $\mathrm{MgO} \ldots \ldots \ldots$ & 1.43 & $\ldots .$. & - \\
\hline $\mathrm{Na}_{2} \mathrm{O}$ & 8.57 & 8.62 & 9.11 \\
\hline $\mathrm{CO}_{2} \quad \therefore \ldots$ & ..... & & .67 \\
\hline $\mathrm{H}_{2} \mathrm{O}$ at $105^{\circ} \ldots \ldots$ & \multirow{3}{*}{4.09} & .04 & .27 \\
\hline $\mathrm{H}_{2} \mathrm{O}, 250^{\circ}-300^{\circ} \ldots \ldots$ & & .14 & 2.70 \\
\hline 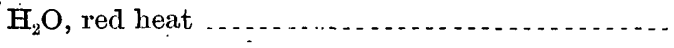 & & 2.86 & \\
\hline . & 100.82 & 99.86 & 100.10 \\
\hline
\end{tabular}




\section{AMPHIBOLE.}

A. Tremolite, pseudomorphous after sahlite; northeast slope of Canaan Mountain, Connecticut. Analysis by W. F. Fillebrand.

B. Tremolite, found in the serpentine of Easton, Pa. Analysis by I. G. Eakins.

C. Actinolite (?), Corundum Hill, North Carolina. Specific gravity, 3.062. Analyzed by T. M. Chatard and described by him in Bulletin 42 .

D. Nephrite, New Zealand. Fragment from a dark-green bowlder. Analysis by F. W. Clarke.

E. Nephrite, Robenhausen, Lake Pfäffikon, Switzerland. Part of a green, compact, highly polished jade implement. Specific gravity, 3.015; determined by William Hallock. Analysis by F. W. Clarke.

\begin{tabular}{|c|c|c|c|c|c|}
\hline . & A. & B. & C. & D. & E. \\
\hline $\mathrm{SiO}_{2}$ & 57.97 & 58.27 & 55.23 & 56.73 & 56.87 \\
\hline $\mathrm{TiO}_{2} \ldots \ldots$ & none & $\ldots$ & none & - & ....... \\
\hline $\mathrm{Al}_{2} \mathrm{O}_{3} \ldots \ldots$ & .09 & .33 & 3.04 & 3.22 & 1.50 \\
\hline $\mathrm{Cr}_{2} \mathrm{O}_{3} \ldots$ & $\ldots$. & $\ldots$ & .19 & 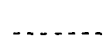 & $\ldots$ \\
\hline $\mathrm{Fe}_{2} \mathrm{O}_{3} \ldots \ldots$ & .11 & $\operatorname{trace} \theta$ & 1.88 & & \\
\hline $\mathrm{FeO} \ldots$ & .18 & $\ldots$ & 2.51 & 5.96 & 6.33 \\
\hline $\mathrm{MnO} \ldots . .$. & trace & .08 & .26 & trace & trace \\
\hline $\mathrm{NiO}, \mathrm{CoO}$ & - & $\ldots$. & trace & $\ldots$ & ..... \\
\hline $\mathrm{CaO} \ldots . . .$. & 15.05 & 11.90 & 13.36 & 13.24 & 13.45 \\
\hline $\mathrm{SrO} \ldots$ & trace & $\ldots \ldots$ & $\ldots$ & -..... & - . \\
\hline $\mathrm{MgO}$.. & 22.45 & 25.93 & 22.31 & 19.42 & 21.06 \\
\hline $\mathrm{K}_{2} \mathrm{O} \ldots$ & .12 & .42 & 8 & undet. & undet. \\
\hline $\mathrm{Na}_{2} \mathrm{O}$ & .20 & 1.25 & & undet. & undet. \\
\hline $\mathrm{H}_{2} \mathrm{O}$ at $100^{\circ} \ldots$ & .03 & 1.22 & .04 & 83 & 63 \\
\hline $\mathrm{H}_{2} \mathrm{O}$ above $100^{\circ}$ & 2.57 & & .52 & .00 & .05 \\
\hline $\mathrm{CO}_{2} \ldots \ldots$ & 1.69 & & . & & \\
\hline & 100.46 & 99.40 & 99.92 & 99.40 & 99.84 \\
\hline
\end{tabular}


F. Dark-green nephrite implement, from the Eskimo of Point Barrow, Alaska. Specific gravity, 3.012. Analysis by F. W. Clarke.

G. Nephrite adze, Point Barrow. Nearly black, with grayish-green patches. Specific gravity, 2.922, Hallock. Analysis by F. W. Clarke.

H. Nephrite adze, Cape Prince of Wales, Alaska. Dark green, laminated in two shades, opaque. Specific gravity, 2.989, Hallock. Analysis by F. W. Clarke.

I. Worked nephrite, St: Michael, Alaska. Dull apple green, fairly uniform in tint, semitranslucent at edges. Specific gravity, 3.006, Hallock. Analysis by F. W. Clarke.

J. Jade implement, Diomede Island, Alaska. Dark green, laminated in two shades, opaque. Specific gravity, 3.010, Hallock. Analysis by F. W. Clarke.

\begin{tabular}{|c|c|c|c|c|c|}
\hline . & F: & G. & H. & I. & .J. \\
\hline $\mathrm{SiO}_{2-}$ & 57.01 & 57.11 & 56.01 & 56.12 & 53.08 \\
\hline $\mathrm{Al}_{2} \mathrm{O}_{3} \ldots$ & .42 & 2.57 & 1.98 & .63 & 1.01 \\
\hline $\mathrm{FeO} \ldots \ldots$ & 6.95 & 5.15 & 6.34 & 7.45 & 7.67 \\
\hline $\mathrm{CaO} \ldots$ & 12.75 & 11.54 & 12.54 & 12.72 & 13.35 \\
\hline $\mathrm{MgO}$ & 21.36 & 21.38 & 21.57 & 20.92 & 19.96 \\
\hline $\mathrm{MnO}_{-}$ & $\therefore--$ & trace & trace & $\operatorname{trace}$ & trace \\
\hline Ignition & 1.41 & 2.06 & 1.91 & 1.42 & 2.03 \\
\hline \multirow[t]{2}{*}{ Alkalies $\ldots \ldots$} & undet. & undet. & undet. & undet. & undet. \\
\hline & 99.90 & 99.81 & 100.35 & 99.26 & 100.10 \\
\hline
\end{tabular}


K, I, M, N. Four samples of nephrite, found in situ by Lieutenant Stoney, U. S. Navy, near the Kowak River, Alaska. K, greenish gray, splintery-lamellar. I, like $\mathrm{K}$, but more granular. $\mathrm{M}$, paler, nearly white, closer grained. $\mathrm{N}$, brownish, highly foliated. Analyses by F. W. Clarke. Ferrous iron determinations by R. B. Riggs. O, Nephrite from Jordansmühl, Silesia; analysis by George Steiger; typical material from an old locality.' For details concerning nephrites D to N, see the memoir by Clarke and Merrill in Proc. U. S. Nat. Mus. for 1888 .

\begin{tabular}{|c|c|c|c|c|c|}
\hline & K. & I. & M. & N. & 0. \\
\hline $\mathrm{SiO}_{2}$ & 58.11 & 55.87 & 56.85 & 57.38 & 56.39 \\
\hline \multicolumn{6}{|l|}{$\mathrm{TiO}_{2}$} \\
\hline $\mathrm{Al}_{2} \mathrm{O}_{3} \ldots$ & .24 & 2.07 & .88 & .19 & 1.63 \\
\hline $\mathrm{Fe}_{2} \mathrm{O}_{3}$ & 5.44 & 5.79 & 4.33 & 4.43 & 1.72 \\
\hline $\mathrm{FeO} \ldots \ldots$ & .38 & .38 & 1.45 & 1.25 & 3.70 \\
\hline $\mathrm{MnO} \ldots \ldots$ & trace & trace & $\operatorname{trace}$ & trace & .26 \\
\hline \multicolumn{6}{|l|}{$\mathrm{NiO} \ldots$} \\
\hline $\mathrm{CaO} \ldots \ldots$ & 12.01 & 12.43 & 13.09 & 12.14 & 7.92 \\
\hline $\mathrm{MgO} \ldots \ldots$ & 21.97 & 21.62 & 21.56 & 22.71 & 24.63 \\
\hline Alkalies & undet. & undet. & undet. & undet. & none \\
\hline \multicolumn{6}{|l|}{$\mathrm{P}_{2} \mathrm{O}_{5}$} \\
\hline \multirow{3}{*}{$\begin{array}{l}\mathrm{H}_{2} \mathrm{O} \text { at } 100^{\circ} \ldots \\
\mathrm{H}_{2} \mathrm{O} \text { above } 100^{\circ} \ldots \ldots\end{array}$} & 178 & 138 & 176 & 178 & .65 \\
\hline & 1.70 & 1.00 & 1.60 & 1.10 & 3.42 \\
\hline & 99.93 & 99.54 & 99.92 & 99.83 & 100.45 \\
\hline
\end{tabular}


P. Brown hornblende, Pierrepont, N. Y. Analysis by T. M. Chatard.

Q. Hornblende separated from gabbro, east'shaft of waterworks extension, Washington, D. C. Analysis by R. B. Riggs.

R. Amphibole from the serpentine of Montville, N. J. Aualysis by I. G. Eakins.

S. Hornblende separation, from south of soapstone quarry, Warwick, Mass. Analysis by E. A. Schneider.

T. Hornblende from amphibolite dike, Palmer Center, Mass. Specific gravity, 3.220 at $31.5^{\circ}$. Analysis by W. F. Hillebrand.

U. Hornblende from amphibolite bed, same locality and analyst. Specific gravity, 3.217 at $29^{\circ}$.

\begin{tabular}{|c|c|c|c|c|c|c|}
\hline & P. & $Q$ & $\mathrm{R}$. & s. & $\mathrm{T}$. & U. \\
\hline $\mathrm{SiO}_{2}$ & 56.44 & 52.42 & 43.31 & 47.86 & 43.11 & 44.09 \\
\hline $\mathrm{TiO}_{2} \ldots \ldots$ & .11 & - & $\ldots$ & a.63 & 1.32 & 1.73 \\
\hline $\mathrm{Al}_{2} \mathrm{O}_{3}$ & 1.77 & 3.15 & 17.41 & 14.09 & 11.10 & 10.68 \\
\hline $\mathrm{V}_{2} \mathrm{O}_{3} \ldots$ &.- & $\cdots$ & 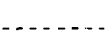 & $\ldots$ & .07 & undet. \\
\hline $\mathrm{Cr}_{2} \mathrm{O}_{3} \ldots \ldots \ldots$ & $\ldots$ & $\ldots$ & $\cdots$ & $\cdots$ & trace & trace \\
\hline $\mathrm{Fe}_{2} \mathrm{O}_{3} \ldots \ldots$ & .84 & 3.60 & .71 & .33 & 4.97 & 2.72 \\
\hline $\mathrm{FeO} \ldots$ & .73 & 8.36 & .59 & 13.41 & 13.04 & 12.96 \\
\hline $\mathrm{MnO} \ldots \ldots \ldots$ & .11 & .11 & .14 & .14 & .43 & .32 \\
\hline $\mathrm{NiO} \ldots$ & $\cdots$ & . & & $\cdots$ & trace & trace \\
\hline $\mathrm{CaO}$ & 11.83 & 14.33 & 12.84 & .57 & 11.76 & 11.58 \\
\hline $\mathrm{MgO} \ldots$ & 22.98 & 15.85 & 19.39 & 19.89 & 9.35 & 10.75 \\
\hline $\mathrm{K}_{2} \mathrm{O}_{\ldots} \ldots$ & .75 & .12 & 1.36 & .06 & 1.27 . & .88 \\
\hline $\mathrm{Na}_{2} \mathrm{O}$ & 2.13 & 1.16 & 2.23 & .93 & 1.18 & 1.19 \\
\hline $\mathrm{H}_{2} \mathrm{O}$ at $100^{\circ} \ldots$ & .05 & 125 & 1.17 & none & .16 & .21 \\
\hline $\mathrm{H}_{2} \mathrm{O}$ above $100^{\circ} \ldots$ & 2.41 & סب 1. & 1.16 & 2.46 & 1.92 & 1.91 \\
\hline \multirow[t]{2}{*}{$\mathrm{P}_{2} \mathrm{O}_{5}$} & trace & & . & .05 & .10 & .10 \\
\hline & 100.15 & 100.35 & 99.15 & 100.42 & 99.78 & 99.12 \\
\hline
\end{tabular}

a Admixed rutile. 
V. Hornblende near barkevikite. Separated from the sodalitesyenite of Square Butte, Highwood Mountains, Montana. Described by Lindgren in Am. Jour. Sci., 3d ser., vol. 45, p. 286. Analysis by W. H. Melville.

W. Amphibole separated from quartz-diorite, south of Table Mountain, on ridge between Butte and Plumas counties, Cal. Described by Turner in Seventeenth Annual Report, Part I, p. 521. Analysis by William Valentine. Chromium determination by Hillebrand.

$X$. Amphibole separated from amphibole gabbro, Beaver Creek, Big Trees quadrangle, California. Analysis by William Valentine. See Bulletin 168, p. 206.

Y. Amphibole separated from quartz-monzonite, Tioga road, southeảst of Mount Floffman, Mariposa County, Cal. Specific gravity, 3.203 at $21.5 .^{\circ}$ Analysis by W. F. Hillebrand. See Bulletin 168, p. 208.

Z. Amphibole separated from the granite of Butte, Mont. Incomplete analysis for lack of material. Analysis by H. N. Stokes.

\begin{tabular}{|c|c|c|c|c|c|}
\hline . & v. & $\mathrm{w}$. & $\mathrm{x}$. & $\mathrm{Y}$. & z. \\
\hline $\mathrm{SiO}_{2} \ldots$ & 38.41 & 50.08 & 46.08 & 47.49 & 45.73 \\
\hline $\mathrm{TiO}_{2} \ldots$ & & .76 & .77 & 1.21 & 1.43 \\
\hline $\mathrm{Al}_{2} \mathrm{O}_{3} \ldots$ & 17.65 & 7.97 & 10.56 & 7.07 & 6.77 \\
\hline $\mathrm{V}_{2} \mathrm{O}_{3} \ldots \ldots$ & $\cdots$ & $\ldots$ & ..... & .04 & $\ldots$ \\
\hline $\mathrm{Cr}_{2} \mathrm{O}_{3} \ldots$ & ........ & .16 & ....... & none & none \\
\hline $\mathrm{Fe}_{2} \mathrm{O}_{3}-$ & 3.75 & 2.69 & 2.81 & 4.88 & 4.94 \\
\hline $\mathrm{FeO} \ldots \ldots$ & 21.75 & 6.71 & 8.30 & 10.69 & 10.39 \\
\hline $\mathrm{MnO} \ldots$ & .15 & .49 & .15 & .51 & .54 \\
\hline $\mathrm{NiO} . .$. & trace & (n) & …... & .02 & 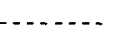 \\
\hline $\mathrm{CaO} \ldots \ldots$ & 10.52 & 11.21 & 12.64 & 11.92 & 11.25 \\
\hline $\mathrm{MgO} \ldots . . .$. & 2.54 & 16.31 & 14.40 & 13.06 & 12.32 \\
\hline $\mathrm{Na}_{2} \mathrm{O}$ & 2.95 & 1.22 & 1.62 & .75 & .77 \\
\hline $\mathrm{K}_{2} \mathrm{O} \ldots$ & 1.95 & .46 & .34 & .49 & 1.22 \\
\hline $\mathrm{Li}_{2} \mathrm{O}$ & $\ldots . .$. & $\cdots$ & none & trace & trace \\
\hline $\mathrm{H}_{2} \mathrm{O}$ at $100^{\circ} \ldots$ & $\ldots$ & - - - & .17 & $\ldots$ & .49 \\
\hline $\mathrm{H}_{2} \mathrm{O}$ above $100^{\circ} \ldots$ & .24 & 1.40 & 1.97 & 1.86 & 2.29 \\
\hline $\mathrm{P}_{2} \mathrm{O}_{5} \ldots \ldots \ldots$ & $\cdots$ & $\operatorname{trace}$ & .18 & none & .35 \\
\hline & …... & undet. & none & .06 & .28 \\
\hline & 99.91 & 99.46 & 99.99 & 100.05 & 98.77 \\
\hline \multirow[t]{2}{*}{$\mathrm{O}$ equivalent to $\mathrm{F}$} & $\ldots \ldots$ & -....... & $\ldots$ & .02 & .12 \\
\hline & & & & 100.03 & 98.65 \\
\hline
\end{tabular}


BERYL.

A. White beryl from the tin mine at Winslow, Me. Opaque, milky. associated with mica, fluorite, calcite, tinstone, etc. Specific gravity, 2.707 at $27^{\circ}$. Analysis by W. F. Hillebrand.

B. Green beryl from near Home post-office in eastern Tennessee. Analysis by F. W. Clarke.

\begin{tabular}{|c|c|c|}
\hline & A. & B. \\
\hline $\mathrm{SiO}_{2 \ldots}$ & 65.21 & 65.39 \\
\hline $\mathrm{TiO}_{2} \ldots \ldots$ & trace & $\cdots$ \\
\hline $\mathrm{Al}_{2} \mathrm{O}_{3} \ldots \ldots$ & 18.50 & 19.10 \\
\hline $\mathrm{Fe}_{2} \mathrm{O}_{3} \ldots \ldots \ldots$ & .33 & \\
\hline $\mathrm{FeO} \ldots$ & & \\
\hline $\mathrm{GlO} \ldots$ & 13.03 & 13.35 \\
\hline $\mathrm{K}_{2} \mathrm{O}, \mathrm{Cs}_{2} \mathrm{O} \ldots$ & .14 & \\
\hline $\mathrm{Na}_{2} \mathrm{O}_{-}$ & .87 & \\
\hline $\mathrm{Li}_{2} \mathrm{O}$ & .16 & \\
\hline $\mathrm{MgO}$ & .09 & \\
\hline \multirow[t]{2}{*}{$\mathrm{H}_{2} \mathrm{O}$} & 1.80 & 1.76 \\
\hline & 100.13 & 99.60 \\
\hline
\end{tabular}

NEPHELITE.

A. Elæolite from Litchfield, Me. Dark-gray cleavable masses, of greasy luster. Described by F. W. Clarke in Bulletin 42.

B. Another sample from Litchfield. Analysis by George Steiger.

C. Nephelite extracted by solution from the elæolite-syenite of Red Hill, N. H. Described by Bayley in Bull. Geol. Soc. America, vol. 3, p. 231. Analysis by W. F. Hillebrand.

\begin{tabular}{|c|c|c|c|}
\hline & : A. & B. & C. \\
\hline $\mathrm{SiO}_{2} \ldots$ & \multirow{2}{*}{$\begin{array}{l}43.74 \\
34.48\end{array}$} & 45.91 & \multirow{2}{*}{$\begin{array}{l}45.31 \\
32.67\end{array}$} \\
\hline $\mathrm{Al}_{2} \mathrm{O}_{3}$ & & 31.14 & \\
\hline $\mathrm{Fe}_{2} \mathrm{O}_{3} \ldots \ldots \ldots$ & ....... & $: 34$ & $\ldots$ \\
\hline $\mathrm{FeO}$ & & .23 & ..... \\
\hline $\mathrm{MgO} \ldots \ldots$ & $\cdots$ & .... & .16 \\
\hline $\mathrm{CaO}$ & trace & .33 & 2.00 \\
\hline $\mathrm{Na}_{2} \mathrm{O}$ & 16.62 & 14.60 & 12.60 \\
\hline $\mathrm{K}_{2} \mathrm{O} \ldots \ldots$ & 4.55 & 5.60 & 5.70 \\
\hline $\mathrm{H}_{2} \mathrm{O}$ at $100^{\circ} \ldots \ldots$ & \multirow{3}{*}{.86} & .47 & $\ldots$ \\
\hline $\mathrm{H}_{2} \mathrm{O}$ above $100^{\circ}$ & & .93 & 1.56 \\
\hline $\mathrm{CO}_{2} \ldots \ldots \ldots$ & & .40 & ................ \\
\hline & 100.25 & 99.95 & 100.00 \\
\hline
\end{tabular}




\section{CANCRINITE.}

All from Litchfield, Me. See description by Clarke in Bulletin 42 .

A. Pale yellow, granular.

B. Bright orange-yellow, cleavable, transparent in thin fragments.

C. Dingy pale yellow, otherwise like $B$.

D. Average yellow sample.

E. Flesh-colored, cleavable mixture of elæolite and cancrinite. Analysis D by George Steiger; the others by F. W. Clarke. $\mathrm{CO}_{2}$ determinations in A, B, C, E by R. B. Riggs.

\begin{tabular}{|c|c|c|c|c|c|}
\hline - & A. & B. & c. & D. & E. \\
\hline $\mathrm{SiO}_{2}$ & 37.22 & 36.29 & 35.83 & 36.19 & 38.93 \\
\hline $\mathrm{Al}_{2} \mathrm{O}_{3}$ & 28.32 & 30.12 & 29.45 & 29.24 & 32.52 \\
\hline $\mathrm{Fe}_{2} \mathrm{O}_{3}$ & trace & trace & $\operatorname{trace}$ & trace & $\ldots$ \\
\hline $\mathrm{MnO}$ & trace & trace & trace & $\ldots$ & trace \\
\hline $\mathrm{CaO}$.- & 4.40 & 4.27 & 5.12 & 4.72 & 2.47 \\
\hline $\mathrm{MgO}_{-}$ & .07 & ....... & 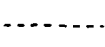 & $\cdots$ & none \\
\hline $\mathrm{Na}_{2} \mathrm{O}$ & 19.43 & 19.56 & 19.33 & 19.20 & 17.02 \\
\hline $\mathrm{K}_{2} \mathrm{O} \ldots$ & .18 &. .18 & .09 & .14 & 3.23 \\
\hline $\mathrm{H}_{2} \mathrm{O} \ldots$ & 3.86 & 2.98 & 3.79 & 4.15 & 2.83 \\
\hline \multirow[t]{2}{*}{$\mathrm{CO}_{2}$} & 6.22 & 6.96 & 6.50 & 6.11 & 2.95 \\
\hline & 99.70 & 100.36 & 100.11 & 99.75 & 99.95 \\
\hline
\end{tabular}




\section{SODALITE.}

A. Blue sodalite, from Litchfield, Me. Analysis by F. W: Clarke. See description in Bulletin 42.

B. Blue sodalite, from Ice River, near Kicking Horse Pass, British Columbia. Analysis by George Steiger:

C. Sodalite separated from the sodalite-syenite of Square Butte, Highwood Mountains, Montana. See Lindgren in Am. Jour. Sci., 3d ser., vol. 45, p. 286. Specific gravity, 2.265. Analysis by W. $\mathbb{H}$. Melville.

\begin{tabular}{|c|c|c|c|}
\hline & A. & B. & c. \\
\hline $\mathrm{SiO}_{2}$ & 37.33 & 39.66 & 41.56 \\
\hline $\mathrm{Al}_{2} \mathrm{O}_{3} \ldots \ldots$ & 31.87 & 30.09 & 29.48 \\
\hline $\mathrm{Fe}_{2} \mathrm{O}_{3}$ & ...... & .31 & -- \\
\hline $\mathrm{FeO} \ldots$ & & 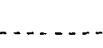 & .49 \\
\hline $\mathrm{MgO}$ & $\cdots$ & $\ldots$ & .15 \\
\hline $\mathrm{CaO}$ & $\cdots$ & .18 & .49 \\
\hline $\mathrm{Na}_{2} \mathrm{O}$ & 24.56 & 22.60 & 19.21 \\
\hline $\mathrm{K}_{2} \mathrm{O} \ldots$ & .10 & 1.14 & .91 \\
\hline $\mathrm{H}_{2} \mathrm{O}$ at $100^{\circ} \ldots \ldots$ & \multirow{2}{*}{1.07} & .17 & .45 \\
\hline $\mathrm{H}_{2} \mathrm{O}$ above $100^{\circ} \ldots \ldots$ & & .79 & 3.73 \\
\hline \multirow[t]{2}{*}{$\mathrm{Cl}$} & 6.83 & 6.12 & 4.79 \\
\hline & 101.76 & 101.06 & 101.26 \\
\hline \multirow[t]{2}{*}{$\mathrm{O}$ equivalent to $\mathrm{Cl}$} & 1.54 & 1.39 & 1.08 \\
\hline & 100.22 & 99.67 & 100.18 \\
\hline
\end{tabular}

ZUNYITE.

From the Zuñi mine, Anvil Mountain, near Silverton, Colo. Analyzed by W. F. Hillebrand, and described by him as a new species in Bulletin 20. Specific gravity, 2.875 at $15^{\circ}$.

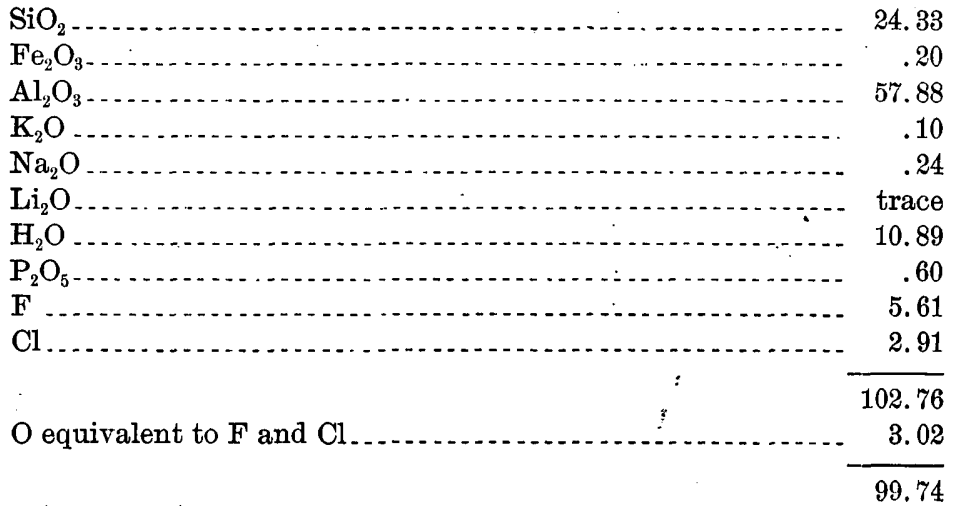


GARNEI.

A. Grossularite. Large waterworn pebble, pale green, very compact. At first, thought to be jade. From Eltoro, 40 miles south of Los Angeles, Cal. Described by Clarke in Am. Jour. Sci., 3d ser., vol. 50; p. 76. Analysis by George Steiger. Specific gravity, 3.485.

B, C, D. Three samples of garnet from Italian Mountain, Gunnison County, Colo. Specific gravities: A, 3.72 at $16^{\circ} ; \mathrm{B}, 3.629$ at $23^{\circ}$; C, 3.721 at $17.2^{\circ}$. Analyses by L. G. Eakins.

E. Pyrope from the peridotite dike of Elliott County, Ky. Described by Diller in Bulletin 38. Analysis by 'T. M. Chatard.

F. Garnets from Hawkes's quarry, Goshen, Mass. Almandite. Analysis by George Steiger.

\begin{tabular}{|c|c|c|c|c|c|c|}
\hline & A. & B. & C. & D. & E. & F. \\
\hline $\mathrm{SiO}_{2-}$ & 37.54 & 37.89 & 39.26 & 36.88 & 41.32 & 37.30 \\
\hline $\mathrm{TiO}_{2} \ldots$ & trace & $\cdots$ & $\ldots$ & $\ldots$ & .16 & .24 \\
\hline $\mathrm{Al}_{2} \mathrm{O}_{3} \ldots$ & 22.84 & 7.90 & 19.63 & 1.0 .34 & 21.21 & 21.84 \\
\hline $\mathrm{Cr}_{2} \mathrm{O}_{3}$ & $\ldots .$. & $\cdots$ & $\cdots$ & $\cdots$ & .91 & .... \\
\hline $\mathrm{Fe}_{2} \mathrm{O}_{3} \ldots$ & .79 & 16.43 & 4.48 & 17.51 & 4.21. & .98 \\
\hline $\mathrm{FeO}$. & .26 & & $\ldots$ & $\ldots . .$. & 7.93 & 32.62 \\
\hline $\mathrm{MnO}$ & $\ldots$ & ....... & trace & $\ldots$ & .34 & 1.86 \\
\hline $\mathrm{CaO}$ & 36.66 & 35.43 & 36.61 & 34.85 & 4.94 & 3.19 \\
\hline $\mathrm{MgO}_{.}$ & .44 & .59 & trace & .43 & 19.32 & 2.50 \\
\hline $\mathrm{Na}_{2} \mathrm{O} \ldots$ & .13 & 1.10 & .16 & trace & .07 & $\ldots$ \\
\hline $\mathrm{K}_{2} \mathrm{O} \ldots$ & & trace & trace & & & \\
\hline $\mathrm{H}_{2} \mathrm{O} \ldots$ & 1.74 & .36 & .08 & .21 & .17 & \\
\hline \multirow[t]{2}{*}{$\mathrm{P}_{2} \mathrm{O}_{5}$} & trace & & 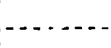 & .. & none & $\ldots$ \\
\hline & 100.40 & 99.70 & 100.22 & 100.22 & 100.58 & 100.53 \\
\hline
\end{tabular}


G. Spessartite from Amelia Court House, Amelia County, Va. Pale brown, crystalline masses. Analyzed by F. W. Clarke and described in Bulletin 60.

H. Spessartite from Llano County, Tex. Yellow, granular. Analyzed by W. H. Melville and described in Bulletin 90. FeO could not be separately determined.

I. Spessartite from cavities in rhyolite, Nathrop, Colo. Brilliant crystals. Specific gravity, 4.23 at $18^{\circ}$. Described by Cross in Am. Jour. Sci., 3d ser., vol. 31, p. 432. Anälysis by I. G. Eakins.

J. Garnet from the Peacock mining claim; Seven Devils mining district, Idaho. Associated with bornite and powellite. Analyzed by W. H. Melville and described in Bulletin 90.

K, L. Two samples andradite from Clifton, Ariz. Analyses by George Steiger.

\begin{tabular}{|c|c|c|c|c|c|c|}
\hline & G. & H. & I. & J. & K. & L. \\
\hline $\mathrm{SiO}_{2}$ & \multirow{2}{*}{35.35} & 35.93 & 35.66 & \multirow{2}{*}{$\begin{array}{r}38.67 \\
-\end{array}$} & 36.26 & \multirow{2}{*}{$\begin{array}{l}42.68 \\
\text { none }\end{array}$} \\
\hline $\mathrm{TiO}_{2} \ldots \ldots$ & & trace & (n...... & & none & \\
\hline $\mathrm{Al}_{2} \mathrm{O}_{3} \ldots$ & 20.41 & 18.08 & 18.55 & \multirow{2}{*}{$\begin{array}{l}10.08 \\
16.00\end{array}$} & .78 & \multirow{2}{*}{$\begin{array}{r}1.53 \\
31.41\end{array}$} \\
\hline $\mathrm{Fe}_{2} \mathrm{O}_{3}$ & \multirow{2}{*}{$\begin{array}{l}2.75 \\
1.75\end{array}$} & \multirow[t]{2}{*}{4.60} & .32 & & 32.43 & \\
\hline $\mathrm{FeO} \ldots \ldots$ & & & 14.25 & .91 & .32 & .30 \\
\hline $\mathrm{MnO}$ & 38.70 & 31.77 & 29.48 & (n) & .27 & .43 \\
\hline $\mathrm{CaO}$ & \multirow{2}{*}{$\begin{array}{c}.94 \\
.\end{array}$} & \multirow{2}{*}{$\begin{array}{r}8.48 \\
\text { trace }\end{array}$} & 1.15 & \multirow[t]{2}{*}{33.35} & 29.67 & \multirow{2}{*}{$\begin{array}{r}.43 \\
23.37\end{array}$} \\
\hline $\mathrm{BaO} \ldots$ & & & & & & \\
\hline $\mathrm{MgO} \ldots \ldots . . . .$. & \multirow[t]{2}{*}{ none } & .69 & . & .77 & none & \multirow{2}{*}{ none } \\
\hline $\mathrm{Na}_{2} \mathrm{O} \ldots$ & & \multirow{2}{*}{.17} & .21 & \multirow{2}{*}{ - } & none & \\
\hline $\mathrm{K}_{2} \mathrm{O} \ldots \ldots$ & & & .27 & & none & . \\
\hline \multicolumn{7}{|l|}{$\mathrm{CuO}$} \\
\hline $\mathrm{P}_{2} \mathrm{O}_{5} \ldots$ & \multirow{3}{*}{.27} & \multirow{3}{*}{$\begin{array}{r}\text { none } \\
.03 \\
.36\end{array}$} & \multirow{3}{*}{.44} & \multirow{3}{*}{.06} & .06 & trace \\
\hline $\mathrm{H}_{2} \mathrm{O}$ at $100^{\circ}$ & & & & & .13 & \\
\hline $\mathrm{H}_{2} \mathrm{O}$ above $100^{\circ}$. & & & & & .44 & 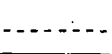 \\
\hline & 100.17 & 100.11 & 100.33 & 99.84 & 100.36 & 99.67 \\
\hline
\end{tabular}




\section{CHRYSOLITE.}

A. Transparent green pebbles from near Fort Wingate, N. Mex. Analysis by E. A. Schneider.

B. Transparent olivine from the meteorite of Kiowa County, Kans. Described in Bulletin 78: Specific gravity; 3.376 at 23.2 . Analysis by L. G. Eakins.

C. Olivine from the peridotite dike of Elliott County, $\mathrm{Ky}$. Described by Diller in Bulletin 38. Analysis by T. M. Chatard.

D. Olivine from the peridotite at Riddle, Oreg. The rock is the matrix of nickel silicates. Described by Diller and Clarke in Bulletin 60. Analysis by F. W. Clarke.

E. Olivine from olivine-gabbro, west side of Birch Lake, Minnesota. Described by Bayley, Jour. Geol., vol. 1, p. 688. Analysis by W. F. Hillebrand.

\begin{tabular}{|c|c|c|c|c|c|}
\hline & A. & B. & c. & $\mathrm{D}$. & $\mathrm{E}$. \\
\hline $\mathrm{SiO}_{2} \ldots$ & 41.98 & 40.70 & 40.05 & 42.81 & 35.58 \\
\hline $\mathrm{TiO}_{2}, \ldots$ & 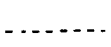 & - n.... & .07 & 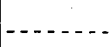 & 1.22 \\
\hline $\mathrm{Al}_{2} \mathrm{O}_{3} \ldots \ldots$ & -- & trace? & .39 & $\cdots$ & .92 \\
\hline $\mathrm{Cr}_{2} \mathrm{O}_{3} \ldots$ & $\cdots$ & $\cdots$ & .24 & .79 & trace \\
\hline $\mathrm{Fe}_{2} \mathrm{O}_{3} \ldots \ldots$ & .51 & .18 & 2.36 & 2.61 & 1 \\
\hline $\mathrm{FeO}$ & 5.71 & 10.79 & 7.14 & 7.20 & 33.91 \\
\hline $\mathrm{MnO}$. & .10 & .14 & .20 & & .35 \\
\hline $\mathrm{NiO} \ldots .$. & .42 & .02 & $-\ldots$ & .26 & .20 \\
\hline $\mathrm{CoO} \ldots$ & $\ldots$ & $\ldots$ & trace & (........ & \\
\hline $\mathrm{MgO} \ldots \ldots$ & 51.11 & 48.02 & 46.68 & 45.12 & 26.86 \\
\hline $\mathrm{CaO} \ldots$ & & & 1.16 & none & .90 \\
\hline $\mathrm{Na}_{2} \mathrm{O} \ldots$ & & & .08 & & $\ldots$ \\
\hline $\mathrm{K}_{2} \mathrm{O} \ldots \ldots \ldots$ & . & 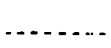 & .21 & $\ldots \ldots$ & $\ldots$ \\
\hline $\mathrm{H}_{2} \mathrm{O}$ at $100^{\circ} \ldots \ldots$ & .05 & & .14 & $5 y$ & .11 \\
\hline $\mathrm{H}_{2} \mathrm{O}$ above $100^{\circ}$. & .23 & & .66 & .07 & .20 \\
\hline \multirow[t]{2}{*}{$\mathrm{P}_{2} \mathrm{O}_{5}$} & $\cdots$ & -...... & .04 & & \\
\hline & 100.11 & 99.85 & 99.42 & 99.36 & 100.25 \\
\hline
\end{tabular}


From a gulch on the side of Italian Mountain, Gunnison County, Colo. Analysis by L. A. Eakins.

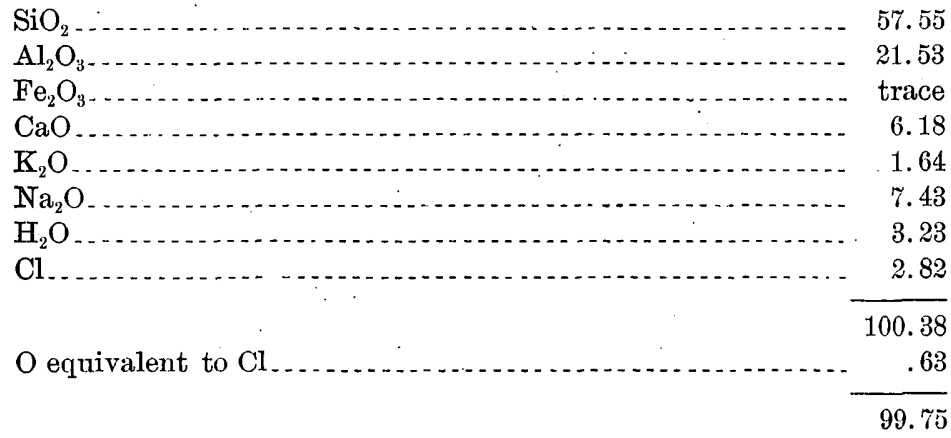

VESUVIANITE.

A. Finely crystallized material from Italian Mountain, Gunnison County, Colo. Analysis by L. G. Eakins. Specific gravity, 3.394 at $20^{\circ}$.

B. From Nevada, 24 kilometers northeast of Silver Peak. Analysis by George Steiger.

C. Pale green, massive. South Fork of Indian Creek, 12 miles from Happy Camp, Siskiyou County, Cal. Analysis by George Steiger.

\begin{tabular}{|c|c|c|c|}
\hline & A. & B. & C. \\
\hline $\mathrm{SiO}_{2} \ldots$ & 37.11 & 36.80 & 35.86 \\
\hline $\mathrm{TiO}_{2} \ldots \ldots$ & (........ & .66 & .10 \\
\hline $\mathrm{Al}_{2} \mathrm{O}_{3} \ldots \ldots \ldots$ & 19.30 & 17.53 & 18.35 \\
\hline $\mathrm{Fe}_{2} \mathrm{O}_{3} \ldots$ & 3.31 & 1.56 & 1.67 \\
\hline $\mathrm{FeO} \ldots \ldots$ & ..... & 3.27 & .39 \\
\hline $\mathrm{MnO} \ldots . .$. & ..... & .48 & .05 \\
\hline $\mathrm{MgO} \ldots \ldots \ldots$ & 3.89 & 1.23 & 5.43 \\
\hline $\mathrm{CaO} \ldots \ldots$ & 36.24 & 35.00 & $33 . \check{1}$ \\
\hline Alkalies ............ & & .13 & none \\
\hline $\mathrm{H}_{2} \mathrm{O}$ at $100^{\circ} \ldots$ & \multirow{4}{*}{.06 . } & .10 & 29 \\
\hline $\mathrm{H}_{2} \mathrm{O}$ above $100^{\circ} \ldots \ldots \ldots$ & & 1.56 & 4.18 \\
\hline $\mathrm{P}_{2} \mathrm{O}_{5} \ldots \ldots$ & & .07 & .02 \\
\hline $\mathrm{CO}_{2}$ & & .65 & none \\
\hline$F$ & .58 & .88 & none \\
\hline \multirow{3}{*}{$O$ equivalent to $F$} & 100.49 & 99.92 & 99.85 \\
\hline & .24 & .36 & $\ldots$. \\
\hline & 100.25 & 99.56 & \\
\hline
\end{tabular}




\section{ZOISITE.}

A. Zoisite from gabbro, east shaft of waterworks extension, Washington, D. C. Analysis by F. W. Clarke; iron determination by R. B. Riggs.

13. Rose-red zoisite, James's mica mine, Yancey County, N. C. Specific gravity, 3.352 at $27^{\circ}$. Analysis by L. G. Eakins.

C. Saussurite from gabbro, Sacramento River road, 37 miles north of Pit River ferry, Shasta County, Cal. Specific gravity, 3.148. Analysis by F. W. Clarke. See Bulletin 9.

D. Saussurite from the Saas Valley, Switzerland. Pale greenish. Specific gravity, 3.37. Analysis by T. W. Clarke; jron determination by R. B. Riggs.

\begin{tabular}{|c|c|c|c|c|}
\hline & A. & B. & C. & D. \\
\hline $\mathrm{SiO}_{2} \ldots \ldots \ldots \ldots \ldots$ & 45.12 & 38.98 & 42.79 & 48. 29 \\
\hline $\mathrm{Al}_{2} \mathrm{O}_{3} \ldots \ldots$ & 30.53 & 31.02 & 29.43 & 27.65 \\
\hline $\mathrm{Fe}_{2} \mathrm{O}_{3}$ & $\therefore-1$ & 4. 1.5 & $\cdots$ & -....... \\
\hline $\mathrm{FeO} \ldots \ldots \ldots$ & 1.90 & $\ldots \ldots$ & 3.65 & 1.45 \\
\hline $\mathrm{MnO}$ & (n) & .23 & -..... & -...... \\
\hline $\mathrm{MgO} \ldots \ldots$. & .42 & - . . . & 1.40 & 5. 36 \\
\hline $\mathrm{CaO} \ldots \ldots . . . .$. & 17.34 & 23.80 & 18.13 & 12.95 \\
\hline $\mathrm{Na}_{2} \mathrm{O} \ldots \ldots$ & 2.02 & $\ldots$ & 2.51 & 3.57 \\
\hline $\mathrm{K}_{2} \mathrm{O} \ldots \ldots$ & 1.09 & ..... & ...... & trace \\
\hline \multirow[t]{2}{*}{$\mathrm{H}_{2} \mathrm{O} \ldots \ldots$} & .74 & 2.03 & 2.42 & .54 \\
\hline & 99.16 & 100.21 & 100.33 & 99.81 \\
\hline
\end{tabular}

Bull. $220-0: 3-4$ 
EPIDOTE.

A. Dark gray, brilliant crystals from Phippsburg, Me. Analyzed by W. F. Hillebrand and described in Bulletin 167.

B, C. Epidote from Italian Mountain, Gunnison County, Colo. Specific gravities: $\mathrm{B}, 3.448$ at $25^{\circ} ; \mathrm{C}, 3.452$ at $17^{\circ}$. Analyses by L. G. Eakins.

\begin{tabular}{|c|c|c|c|}
\hline & A. & B. & c. \\
\hline $\mathrm{SiO}_{2} \ldots$ & 38.54 & 38.21. & 37.92 \\
\hline $\mathrm{Al}_{2} \mathrm{O}_{3} \ldots \ldots$ & 28.39 & 28.70 & 24.09 \\
\hline $\mathrm{Fe}_{2} \mathrm{O}_{3}$ & 6.89 & 8.16 & 12.80 \\
\hline $\mathrm{FeO}: \ldots \ldots$ & .50 & $\ldots$ & .79 \\
\hline $\mathrm{MnO} \ldots$ & . & $\ldots$ & .11 \\
\hline $\mathrm{MgO}$ & trace & $\ldots$ & trace \\
\hline $\mathrm{CaO} \ldots .$. & 24.12 & 24.30 & 23.36 \\
\hline $\mathrm{Na}_{2} \mathrm{O}$ & $\ldots$ & .21 & .06 \\
\hline $\mathrm{H}_{2} \mathrm{O} \ldots \ldots$ & 2.26 & .10 & 1.61 \\
\hline \multirow[t]{2}{*}{$\mathrm{F}$} & none & .35 & .06 \\
\hline & 100.70 & 100.03 & 100.10 \\
\hline \multirow[t]{2}{*}{ O equivalent to $F$} & & .15 & .02 \\
\hline & & 99.88 & 100.08 \\
\hline
\end{tabular}

PIEDMONTITE.

From the rhyolite of Pine Mountain, near Monterey Station, Maryland. Contains a little admixed quartz. Analysis by W. F. Hillebrand.

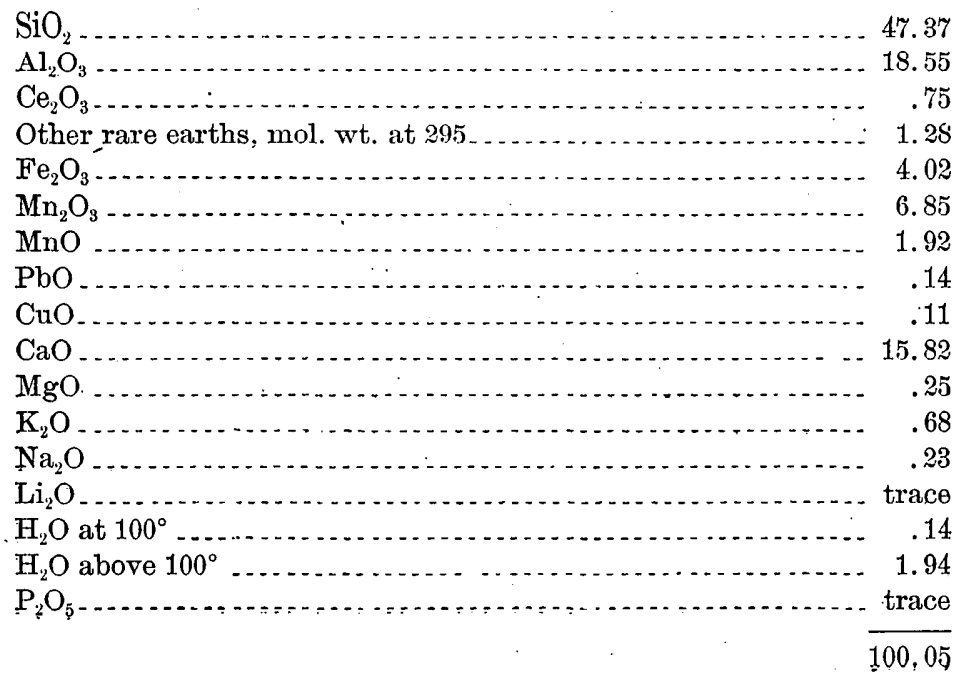




\section{ALLANITE.}

From Platte Mountain, Douglas County, Colo. Specific gravity, 3.52 at $29^{\circ}$. Analysis by L. G. Eakins. See Proc. Colorado Sci. Soc., vol. 2, p. 32 .

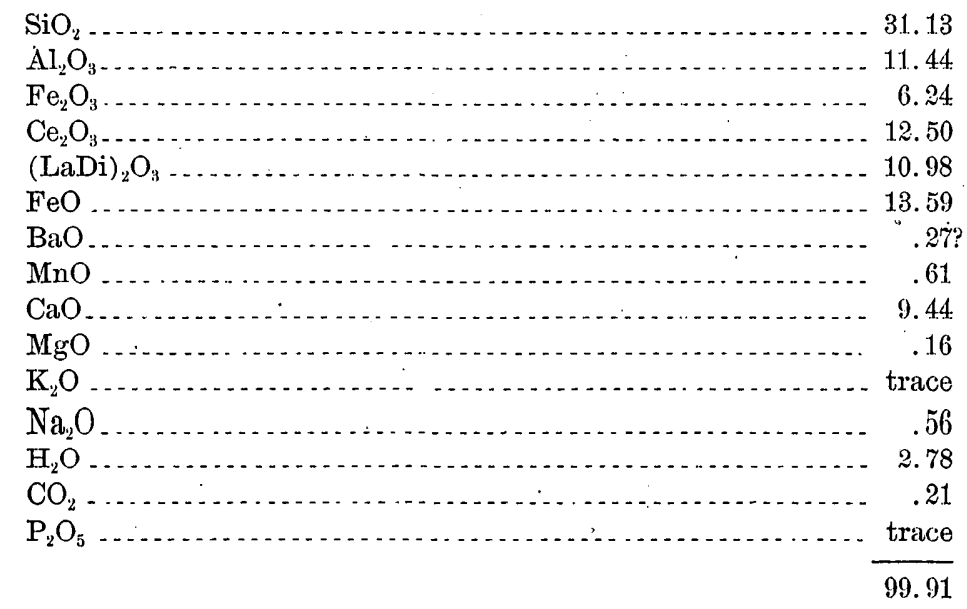

PREHNITE.

A. From Paterson, N. J. Analysis by George Steiger.

B. From Fassa, Tyrol. Analysis by E. A. Schneider.

\begin{tabular}{|c|c|c|}
\hline & A. & B. \\
\hline $\mathrm{SiO}_{2} \ldots \ldots$ & 42.31 & 43.32 \\
\hline $\mathrm{Al}_{2} \mathrm{O}_{3} \ldots \ldots$ & 19.95 & 25.50 \\
\hline $\mathbf{F e}_{2} \mathrm{O}_{3} \ldots \ldots \ldots \ldots \ldots \ldots$ & 6.20 & $\operatorname{trace}$ \\
\hline $\mathrm{FeO} \ldots \ldots \ldots$ & none & .......... \\
\hline $\mathrm{CaO} \ldots \ldots$ & 26.63 & 26.49 \\
\hline $\mathrm{MgO} \ldots$ & nọe & $\ldots$. \\
\hline $\mathrm{H}_{2} \mathrm{O}$ at $100^{\circ} \ldots$ & .21 & .17 \\
\hline $\mathrm{H}_{2} \mathrm{O}$ at $250^{\circ}-300^{\circ} \ldots$ & 481 & .14 \\
\hline $\mathrm{H}_{2} \mathrm{O}$ by ignition $\ldots$ & 4.81 & 4.70 \\
\hline • & 100.11 & 100.32 \\
\hline
\end{tabular}


TOPAZ.

A. White, opaque topaz from Stoneham, Me. Specific gravity, 3.51 .

33. Alteration product of A, greenish. Specific gravity, 3.42 . Analyses A and B by J. E. Whitfield. See discussion by Clarke in Bulletin 27.

C. Topaz from Florissant, Colo. Specific gravity, 3.578 at $22^{\circ}$. Analyzed by W. F. Hillebrand, and described in Bulletin 20.

\begin{tabular}{|c|c|c|c|}
\hline & A. & B. & C. \\
\hline $\mathrm{SiO}_{2} \ldots \ldots$ & 31.92 & 35.15 & 33.15 \\
\hline $\mathrm{Al}_{2} \mathrm{O}_{3} \ldots \ldots$ & 57.38 & 53.18 & $5 \% .01$ \\
\hline $\mathrm{CaO} \ldots .$. & 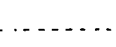 & 1. 32 & - \\
\hline $\mathrm{MgO}_{\text {- }}$ & $\ldots$ & .17 & 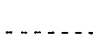 \\
\hline $\mathrm{K}_{2} \mathrm{O} \ldots$ & .15 & 1.52 & -1 \\
\hline $\mathrm{Na}_{2} \mathrm{O}$ & 1.33 & 1.28 & \\
\hline $\mathrm{H}_{2} \mathrm{O}$ & .20 & .90 & $\cdots$ \\
\hline \multirow[t]{2}{*}{$F$} & 16.99 & 12.88 & 16.04 \\
\hline & 107.97 & 106.40 & 103.20 \\
\hline \multirow[t]{2}{*}{ O equivalent to $\mathrm{F}_{\ldots} \ldots \ldots \ldots \ldots \ldots$} & 7.16 & 5.42 & 6.75 \\
\hline & 100.81 & 100.98 & 99.45 \\
\hline
\end{tabular}

The final alteration of the Stoneham topaz is into muscovite, q. v. The alteration product here given represents the beginning of the process.

\section{SILLIMANITE.}

Fibrolite adze from Brittany. Specific gravity, 3.147, determined by William Hallock. Analysis by F. W. Clarke. See Bulletin 60 .

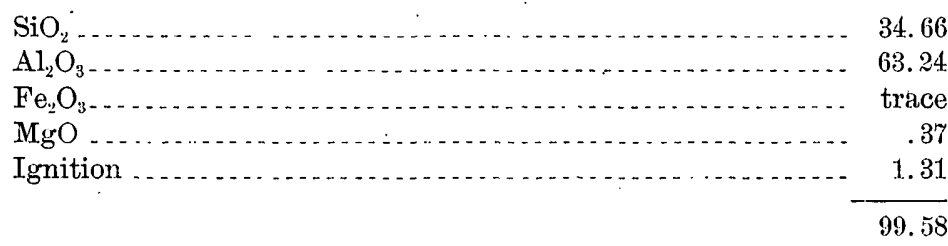

KYANITE.

Pale green variety, associated with the dumortierite of Clip, $\Lambda$ riz. Analyzed by W. F. Hillebrand. Titanic oxide is present in appreciable amounts, but was not separated from alumina. The mineral contained a few black, nonmagnetic grains, which may have carried the titanium. Specific gravity, 3.656 at $18.5^{\circ}$. 


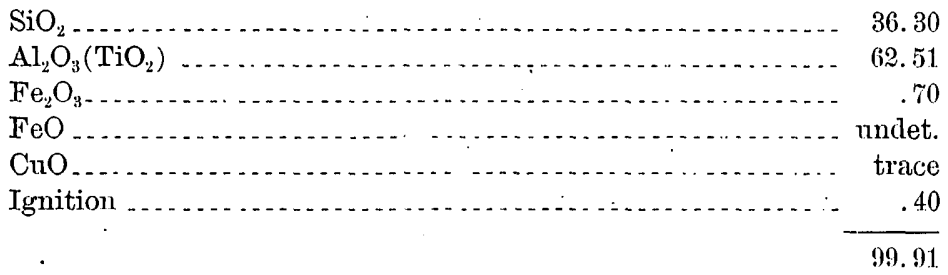

IILVATTE.

From Golconda mine, South Mountain, Owyliee County, Iraho. Analysis by W. F. Hillebrand. Specific gravity, 4.059 at $.31^{\circ}$.

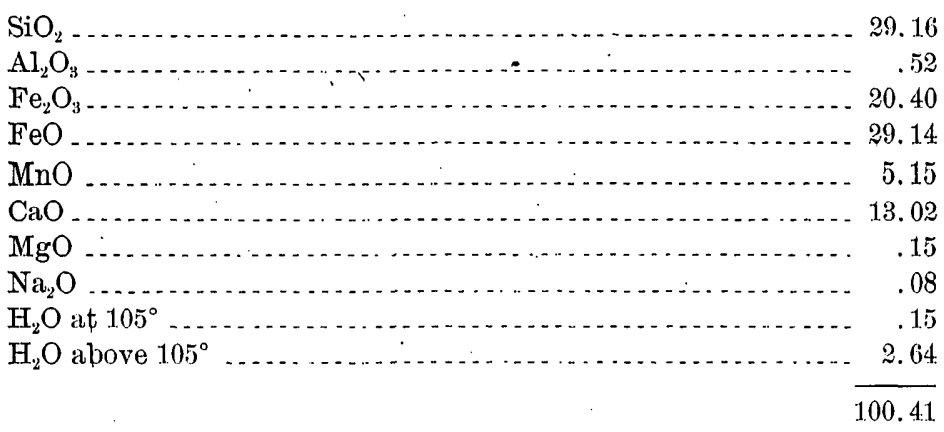

CATAMINE.

White, highly crystalline. From Sterling, N. J. Analysis by George Steiger.

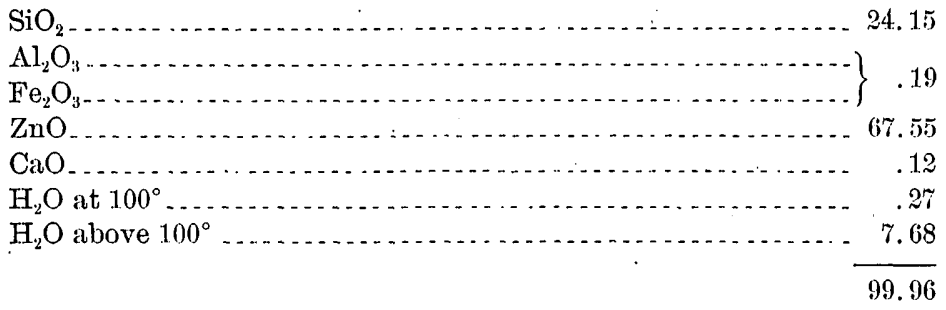

L.AWSONITE.

From Tiburon Peninsula, California. Collected by F. I. Ransome. Analysis by W. F. Hillebrand.

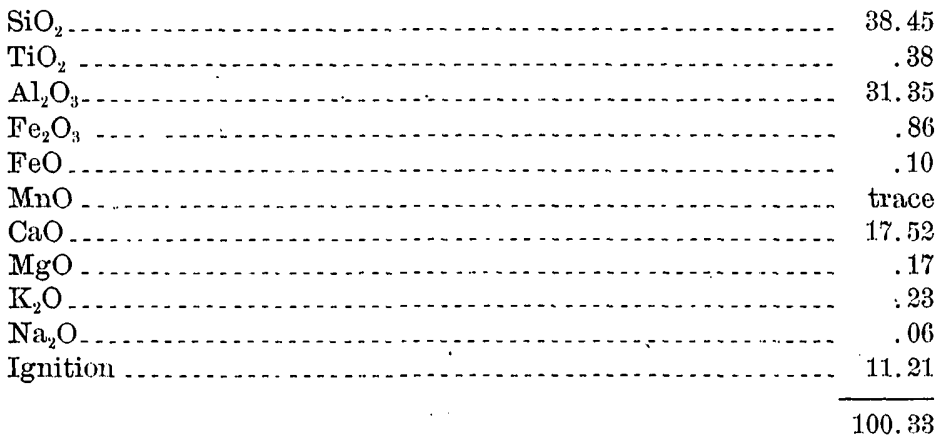


STAUROLITE.

Altered staurolite from Liberty Grove, Cecil County, Md. About two-thirds muscovite. Analysis by George Steiger.

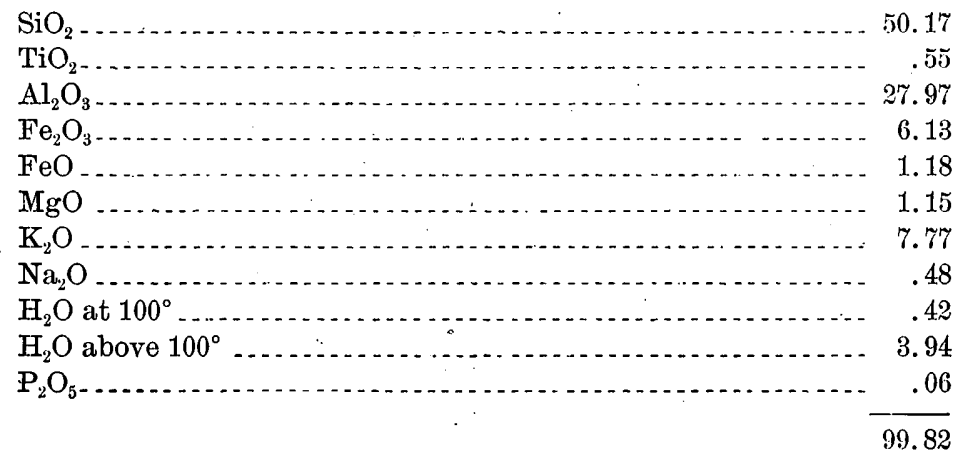

GADOLINITE.

Analyses by T. G. Eakins.

A. From Llano County, Tex. Specific gravity, 4.239 at $17.4^{\circ}$.

B, C. From Devils Head Mountain, Douglas County, Colo. Specific gravities: $\mathrm{B}, 4.56$ at $17^{\circ} ; \mathrm{C}, 4.59$ at $25.5^{\circ}$. Described in Proc. Colorado Sci. Soc., vol. 2 , pt. 1, p. 32 .

\begin{tabular}{|c|c|c|c|}
\hline & A. & B. & C. \\
\hline $\mathrm{SiO}_{2}$ & 23.79 & 22.13 & 21.86 \\
\hline $\mathrm{ThO}_{2} \ldots \ldots$ & .58 & .89 & .81 \\
\hline $\mathrm{Al}_{2} \mathrm{O}_{3} \ldots$ & - & 2.34 & .54 \\
\hline $\mathrm{Fe}_{2} \mathrm{O}_{3} \ldots$ & .96 & 1.13 & 3.59 \\
\hline $\mathrm{Ce}_{2} \mathrm{O}_{3}$ & 2.62 & 11.10 & 6.87 \\
\hline$(\mathrm{La} \mathrm{Di})_{2} \mathrm{O}_{3}$ & 5.22 & 21.23 & 19. 10 \\
\hline $\mathrm{Y}_{2} \mathrm{O}_{3} \ldots \ldots$ & $a 41.55$ & $b 9.50$ & $b 12.63$ \\
\hline $\mathrm{Er}_{2} \mathrm{O}_{3}$ & & $b 12.74$ & $b 15.80$ \\
\hline $\mathrm{FeO} \ldots$ & 12.42 & 10.43 & 11.36 \\
\hline $\mathrm{MnO} \ldots$ & trace & $\ldots$ & .11 \\
\hline G1O & 11.33 & 7.19 & 5.46 \\
\hline $\mathrm{CaO} \ldots \ldots$ & .74 & .34 & .47 \\
\hline $\mathrm{MgO}$ & trace & .14 & .16 \\
\hline $\mathrm{K}_{2} \mathrm{O} \ldots \ldots$ & trace & .18 & .20 \\
\hline $\mathrm{Na}_{2} \mathrm{O}$ & trace & .28 & .32 \\
\hline $\mathrm{H}_{2} \mathrm{O}$ & 1.03 & .86 & .74 \\
\hline \multirow[t]{2}{*}{$\mathrm{P}_{2} \mathrm{O}_{5}$} & .05 & & \\
\hline & 100.29 & 100.48 & 100.02 \\
\hline
\end{tabular}

a Molecular weight, 260.

$\checkmark$ Molecular weight Y, Er group: in B, 296; in C, 294. 


\section{YTTRIALITE.}

From the Barringer mine, Llano County, Tex. Analyzed by W. F. Hillebrand, and discussed in Amer. Jour. Sci., 4th ser., vol. 13, p. 145.

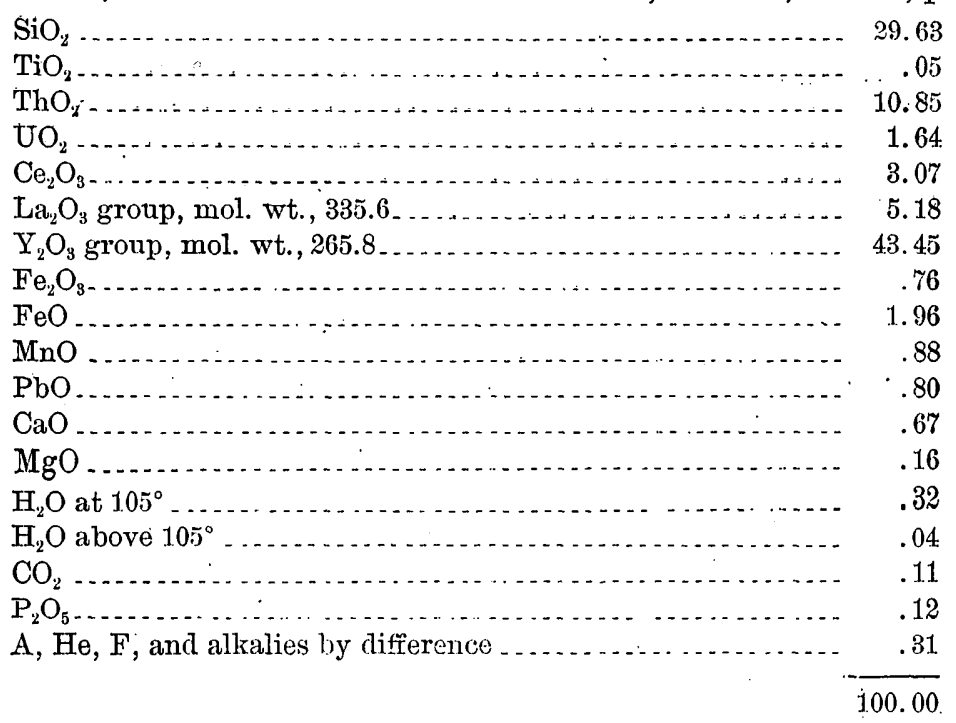

\section{ROWLANDITE.}

From Llano County, Tex. Analysis by W. F. Hillebrand, with discussion in Bulletin 113. Specific gravity, 4.513 at $15.5^{\circ}$.

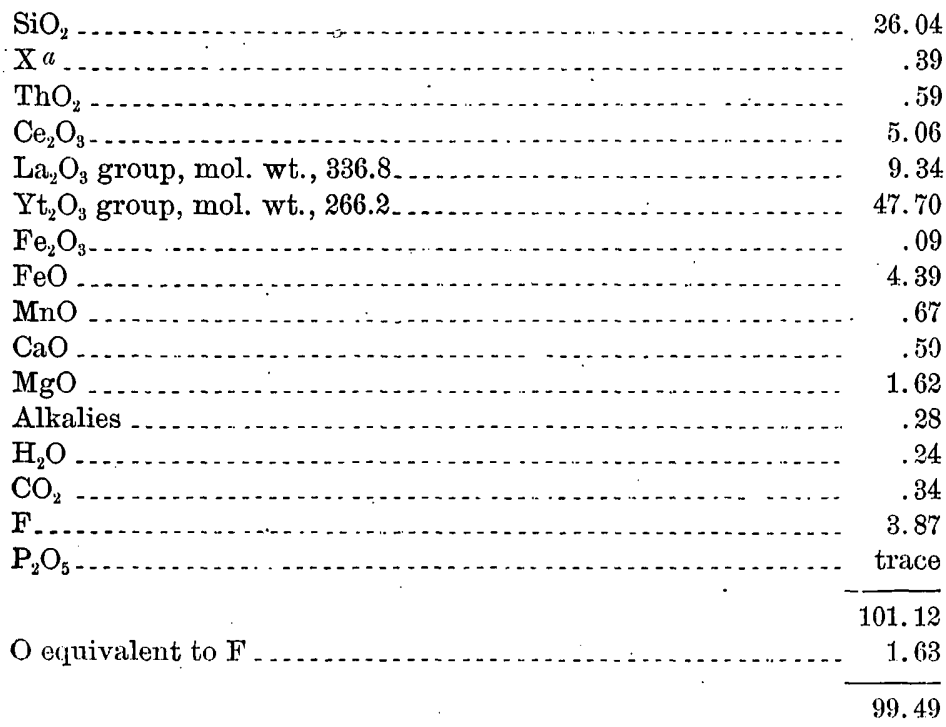

MACKINTOSHITE.

From Llano County, Tex. Analyzed by W. F. Hillebrand and discussed in Bulletin 113. Specific gravity, 5.43 at $21.4^{\circ}$. Only ninetenths of a gram available for analysis. 


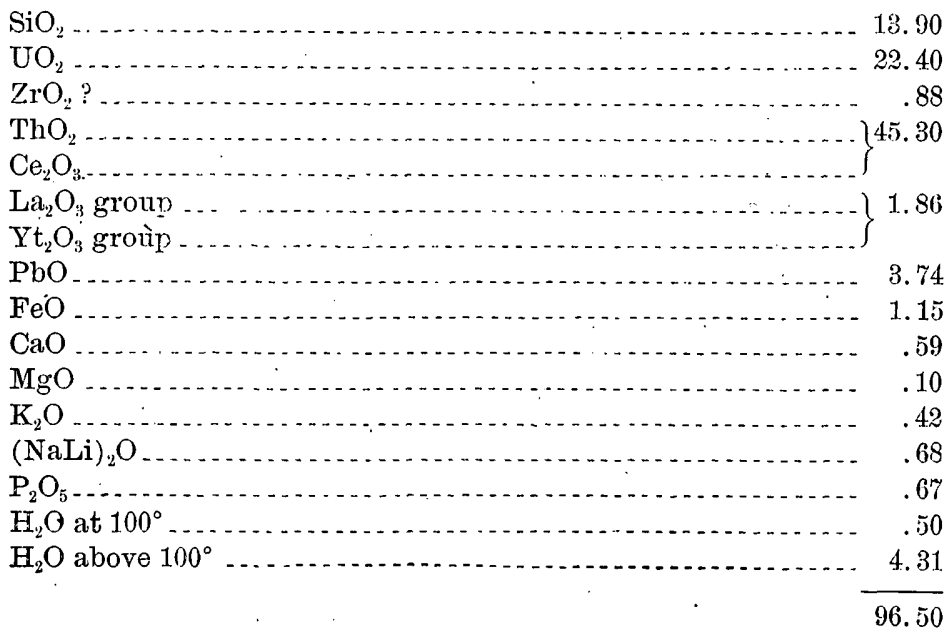

CYRTOLITE.

A doubtful mineral from Devils Head Mountain, Douglas County, Colo. Analyses by W. F. Hillebrand, with description in Proc. Colorado Sci. Soc., vol. 3, pt. 1, p. 44. Brown crystalline growths of irregular form. Specific gravities: A, 3.70; B, 3.60; C, 3.64. The material may be a mixture of cyrtolite or some analogous alteration of zircon with limonite and a phosphate.

\begin{tabular}{|c|c|c|c|}
\hline & $\Lambda$. & B. & C. \\
\hline $\mathrm{SiO}_{2-}$ & 20.06 & 20.64 & 19.21 \\
\hline $\mathrm{Ta}_{2} \mathrm{O}_{5}$ & & .71 & \\
\hline $\mathrm{SnO}_{2} \ldots \ldots \ldots$ & 47.99 & .03 & 51.00 \\
\hline $\mathrm{ZrO}_{2} \ldots$ & & 47.81 & \\
\hline $\mathrm{ThO}_{2} \ldots \ldots \ldots$ & 1.16 & & \\
\hline $\begin{array}{l}\mathrm{Ce}_{2} \mathrm{O}_{3} \\
(\mathrm{LaDi})_{2} \mathrm{O}_{3} \ldots \ldots\end{array}$ & $\begin{array}{l}.06 \\
.19\end{array}$ & 1.20 & .60 \\
\hline $\mathrm{Er}_{2} \mathrm{O}_{3} \ldots \ldots \ldots$ & 4.77 & 4.76 & 4.55 \\
\hline $\mathrm{Y}_{2} \mathrm{O}_{3}$ & 2.27 & 2.48 & 3.18 \\
\hline $\mathrm{Fe}_{2} \mathrm{O}_{3}$ & 5.53 & $5.9 \%$ & 4.86 \\
\hline $\mathrm{MnO}$ & $\therefore 47$ & .57 & .33 \\
\hline $\mathrm{CaO}$ & 1.99 & 1.93 & 2.15 \\
\hline $\mathrm{MgO}$ & .13 & .11 & $\cdots$ \\
\hline $\mathrm{K}_{2} \mathrm{O} \ldots \ldots$ & .20 & .10 & .17 \\
\hline $\mathrm{Na}_{22} \mathrm{O}$ & .46 & .50 & .42 \\
\hline $\mathrm{H}_{2} \mathrm{O} \ldots \ldots$ & 12.87 & 12.00 & 12.97 \\
\hline $\mathrm{P}_{2} \mathrm{O}_{5}$ & 1.64 & 1.75 & .93 \\
\hline \multirow{2}{*}{ 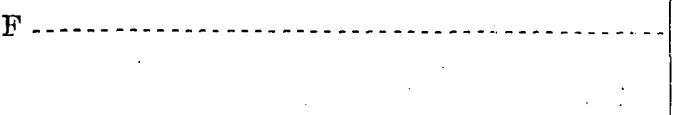 } & .25 & .42 & .42 \\
\hline & 100.04 & 100.98 & 100.74 \\
\hline
\end{tabular}




\section{DANBURI'IE.}

From Russell, N. Y. Analysis by .T. E. Whitfield. See Bulletin 55.

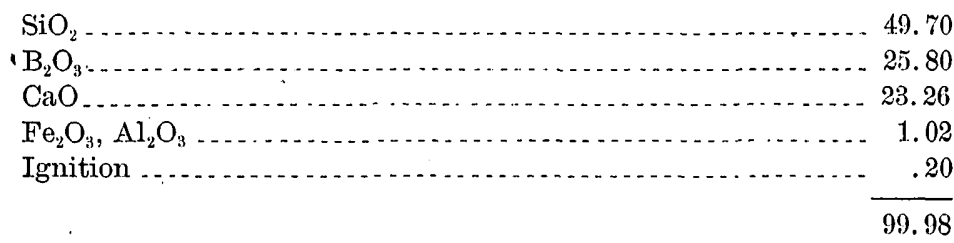

DATOLITE.

From Bergen Hill, N. J. Analysis by J. E. Whitfield. See Bulletin 55 .

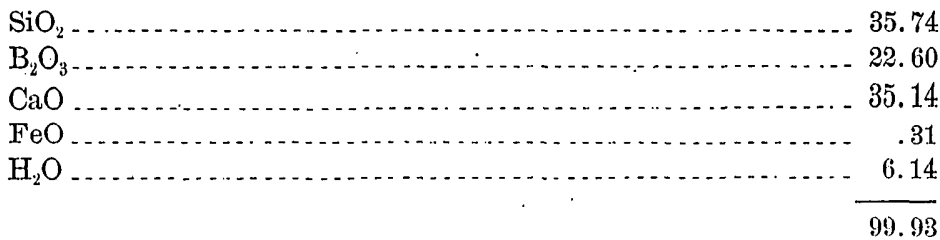

AXINITE.

Analyses by J. E. Whitfield, with discussion in Bulletin 55 .

A. Clove brown, from Cornwall, Ëngland. Translucent.

B. Pearl gray, from Bourg d'Oisans, Dauphiny. Transparent.

\begin{tabular}{|c|c|c|}
\hline 0 & A. & B. \\
\hline $\mathrm{SiO}_{2,-}$ & 42.10 & 41.53 \\
\hline $\mathrm{Al}_{3} \mathrm{O}_{3} \ldots \ldots$ & 17.40 & 17.90 \\
\hline $\mathrm{Fe}_{2} \mathrm{O}_{3}, \ldots \ldots \ldots \ldots$ & 3.06 & 3.90 \\
\hline $\mathrm{FeO} \ldots \ldots . . .$. & 5.84 & 4.02 \\
\hline $\mathrm{CaO} \ldots \ldots \ldots$ & 20.53 & 21.66 \\
\hline $\mathrm{MnO} \ldots \ldots . . . . . . .$. & -4.63 & 3.79 \\
\hline 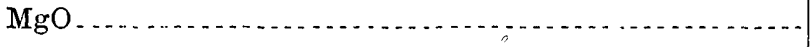 & .66 & .74 \\
\hline $\mathrm{B}_{8} \mathrm{O}_{3} \ldots \ldots \ldots \ldots$ & 4.64 & 4.62 \\
\hline \multirow[t]{2}{*}{$\mathrm{H}_{2} \mathrm{O} \ldots \ldots$} & 1.80 & 2.16 \\
\hline & 100.66 & 100.32 \\
\hline
\end{tabular}


DUMORTIERITE.

A. Harlem, N. Y. Analysis by R. B. Riggs. See Am. Jour. Sci., 3d ser., vol. 34, p. 406.

B. Harlem, N. Y. Analysis by J. E. Whitfield.

C. Near Clip, Yuma County, Ariz. Analysis by J. E. Whitfield.

D. Purified sample from Clip. Analysis by Whitfield. Specific gravity a little over 3.265 .

See note by Whitfield in Bulletin 60, and paper by Diller and Whitfield in Bulletin 64.

\begin{tabular}{|c|c|c|c|c|}
\hline & A. $a$ & B. & C. & D. \\
\hline $\mathrm{SiO}_{2} \ldots \ldots$. & 34.82 & 31.44 & 31.52 & $2 r .99$ \\
\hline $\mathrm{Al}_{2} \mathrm{O}_{3} \ldots$ & 55.30 & 68.91 & 63.66 & 64.49 \\
\hline $\mathrm{Fe}_{2} \mathrm{O}_{3}$ & $\operatorname{trace}$ & & $\ldots$ & , \\
\hline $\mathrm{CaO} \ldots \ldots . . . . .$. & trace & ... & trace & $\cdots$ \\
\hline $\mathrm{MgO}$ & .57 & & .52 & trace \\
\hline $\mathrm{Na}_{2} \mathrm{O}$ & 1.76 & $\ldots$. & .37 & 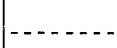 \\
\hline $\mathrm{K}_{2} \mathrm{O}$ & 1.04 & & .11 & ....... \\
\hline $\mathrm{Li}_{2} \mathrm{O} \ldots \ldots$ & trace & & .... & $\ldots$ \\
\hline $\begin{array}{lll} & -1\end{array}$ & 4.07 & trace & 2.62 & 4.95 \\
\hline $\mathrm{P}_{2} \mathrm{O}_{5}$ & $\ldots$ & & $\ldots$ & .20 \\
\hline \multirow{2}{*}{ Ignition $\ldots \ldots \ldots \ldots \ldots$} & 2.96 & (n..... & 1.34 & 1.72 \\
\hline & 100.52 & 100.35 & 100.14 & 99.35 \\
\hline
\end{tabular}

“Sample A contaminated by tourmaline. 


\section{TOURMALINE.}

Analyses A to V, inclusive, by R. B. Riggs. Discussed by Riggs in Bulletin 55.

A. Pink center of crystals having a green margin, from Calhao, province of Minas-Geraes, Brazil. Specific gravity, 3.028.

B. Pale green, border of $A$.

C. Olive-green, also from Calhao, Brazil.

D. Rose-colored, massive, from Black Mountain, Rumford, Me. Specific gravity, 2.997.

E. Dark green, massive, same locality as D.

\begin{tabular}{|c|c|c|c|c|c|}
\hline & A. & B. & C. & D. & $\mathrm{E}$ \\
\hline $\mathrm{SiO}_{2 \ldots}$ & 37.19 & 37.39 & 36.91 & 38.07 & 36.53 \\
\hline $\mathrm{Al}_{2} \mathrm{O}_{3}$ & 42.43 & 39.65 & 38.13 & 42.24 & 38.10 \\
\hline $\mathrm{Fe}_{2} \mathrm{O}_{3}$ & none & .15 & .31 & $\cdots$ & none \\
\hline $\mathrm{FeO}_{-}$. & .52 & 2.29 & 3.19 & .26 & 6.43 \\
\hline $\mathrm{MnO}$ & .79 & 1.47 & 2.22 & .35 & .32 \\
\hline $\mathrm{MgO}$ & none & none & .04 & .07 & none \\
\hline $\mathrm{CaO}_{-}$. & .57 & .49 & .38 & .56 & .34 \\
\hline $\mathrm{Li}_{2} \mathrm{O} \ldots \ldots$ & 1.73 & 1.71 & 1.61 & 1.59 & .95 \\
\hline $\mathrm{Na}_{22} \mathrm{O}$ & 2.24 & 2.42 & 2.70 & 2. 18 & 2.86 \\
\hline $\mathrm{K}_{2} \mathrm{O} \ldots$ & .23 & .25 & .28 & .44 & $: 38$ \\
\hline $\mathrm{P}_{2} \mathrm{O}_{5}$ & none & trace & .11 & none & trace \\
\hline $\mathrm{B}_{2} \mathrm{O}_{3} \ldots$ & 10.06 & 10.29 & 9.87 & 9.99 & 10.22 \\
\hline $\mathrm{H}_{2} \mathrm{O} \ldots$ & 3.90 & $\cdot 3.63$ & 3.64 & 4.26 & 3.52 \\
\hline \multirow{4}{*}{ O equivalent to $F_{\ldots}$} & trace? & .32 & .14 & .28 & .16 \\
\hline & 99.66 & 100.06 & 99.53 & 100.29 & 99.81 \\
\hline & & .13 & .06 & .12 & .07 \\
\hline & & 99.93 & 99.47 & 100.17 & 99.74 \\
\hline
\end{tabular}


F. Alteration product of the Rumford tourmaline.

G. Alteration product of rubellite, from Hebron, Me.

H. Colorless to very pale pinkish or greenish crystals, Auburn, Me. Specific gravity, 3.07 .

I. Light-green crystals, Anburn, Me.

J. Dark green, massive tourmaline, Arburn, Me.

\begin{tabular}{|c|c|c|c|c|c|}
\hline & $\mathrm{F}$. & G. & H. & I. & $\mathrm{T}$. \\
\hline $\mathrm{SiO}_{2-}$ & 53.03 & 43.90 & 38.14 & 37.85 & 36.26 \\
\hline $\mathrm{Al}_{2} \mathrm{O}_{3} \ldots$ & 31.67 & 38.71 & 39.60 & 37.73 & 36.68 \\
\hline $\mathrm{Fe}_{2} \mathrm{O}_{3} \ldots \ldots$ & .51 & .58 & .30 & .42 & .15 \\
\hline $\mathrm{FeO} \ldots$ & $\ldots . .$. & .25 & 1.38 & 3.88 & 7.07 \\
\hline $\mathrm{MnO} \ldots$ & trace & .04 & 1. 38 & .51 & .72 \\
\hline $\mathrm{MgO}$ & trace & .05 & trace & .04 & .16 \\
\hline $\mathrm{CaO} \ldots$ & trace & .41 & .43 & .49 & .17 \\
\hline $\mathrm{Li}_{2} \mathrm{O}$ & .26 & $\ldots$ & 1.34 & 1.34 & 1.05 \\
\hline $\mathrm{Na}_{2} \mathrm{O}$ & .54 & 1.05 & 2.36 & 2.16 & 2.88 \\
\hline $\mathrm{K}_{2} \mathrm{O} \ldots \ldots \ldots$ & 9.44 & 10.92 & .27 & .62 & .44 \\
\hline $\mathrm{B}_{2} \mathrm{O}_{3}$ & trace & trace & 10.25 & 10.55 & 9.94 \\
\hline $\mathrm{P}_{2} \mathrm{O}_{5} \ldots$ & $\ldots \ldots$ & $\ldots$ & trace & trace & trace \\
\hline $\mathrm{H}_{2} \mathrm{O} \ldots$ & 4.80 & 4.25 & 4.16 & 4. 18 & 4.05 \\
\hline \multirow[t]{2}{*}{ F } & trace? & none & .62 & .62 & .71 \\
\hline & 100.25 & 100.16 & 100.23 & 100.39 & 100.28 \\
\hline \multirow[t]{2}{*}{$\mathrm{O}$ equivalent to $\mathrm{F}$} & - & L & .26 & .26 & .30 \\
\hline & & & 99.97 & 100.13 & 99.98 \\
\hline
\end{tabular}


K. White to light-brown crystals, Dekalb, St. Lawrence County, N. Y. Specific gravity, 3.085.

I. Brown tourmaline, Gouverneur, St. Lawrence County, N. Y.

M. Cinnamon-brown crystals, Hamburg, N. J.

N. Brilliant black crystals, Pierrepont, St. Lawrence County, N. Y. Specific gravity, 3.08.

O. Dark-brown erystals, Orford, N. H.

P. Dark-brown crystals, Monroe, Conn.

\begin{tabular}{|c|c|c|c|c|c|c|}
\hline & $\mathrm{k}$. & L. & $M$. & N. . & 0. & P. \\
\hline $\mathrm{SiO}_{2-}$. & $36.88^{\circ}$ & 37.39 & 35.25 & 35.61 & 36.66 & 36.41 \\
\hline $\mathrm{TiO}_{2} \ldots \ldots$ & .12 & 1.19 & .65 & .55 & .23 & 1.61 \\
\hline $\mathrm{Al}_{2} \mathrm{O}_{3} \ldots \ldots$ & 28.87 & 27.79 & 28.49 & 25.29 & 32.84 & 31.27 \\
\hline $\mathrm{Fe}_{2} \mathrm{O}_{3}-$ & 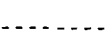 & .10 & none & .44 & none & none \\
\hline $\mathrm{FeO}$ & .52 & .64 & .86 & 8.19 & 2.50 & 3.80 \\
\hline $\mathrm{MnO}$ & none & none & none & $\operatorname{trace}$ & trace & trace \\
\hline $\mathrm{MgO} .$. & 14.53 & 14.09 & 14.58 & 11.07 & 1.0 .35 & 9.47 \\
\hline $\mathrm{CaO} \ldots$ & 3.70 & 2.78 & 5.09 & 3. 31 & 1.35 & .98 \\
\hline $\mathrm{SrO}$ & trace & trace & trace & none & trace & trace \\
\hline $\mathrm{Li}_{2} \mathrm{O} \ldots$ & trace & trace & trace & trace & trace & none \\
\hline $\mathrm{Na}_{2} \mathrm{O}_{\ldots} \ldots$ & 1.39 & 1.72 & .94 & 1.51 & 2.42 & 2.68 \\
\hline $\mathrm{K}_{2} \mathrm{O} \ldots \ldots$ & .18 & .16 & .18 & .20 & .22 & .21 \\
\hline $\mathrm{B}_{2} \mathrm{O}_{3} \ldots$ & 10.58 & 10.73 & 10.45 & 10.15 & 10.07 & 9.65 \\
\hline $\mathrm{P}_{2} \mathrm{O}_{5} \ldots \ldots$ & undet. & none & trace & trace & none & trace \\
\hline $\mathrm{H}_{2} \mathrm{O} \ldots$ & 3.56 & 3.83 & 3.10 & 3.34 & 3.78 & 3.79 \\
\hline \multirow[t]{2}{*}{$F$} & .50 & trace? & .78 & .27 & trace? & none \\
\hline & 100.83 & 100.42 & 100.37 & 99.93 & 100.42 & 99.87 \\
\hline \multirow[t]{2}{*}{ O equivalent to $\mathrm{F}$. } & .21 & $\ldots$ & .33 & .11 & & \\
\hline & 100.62 & & 100.04 & 99.82 & & \\
\hline
\end{tabular}


Q. Massive, black tourmaline, Auburn, Me. Specific gravity, 3.19.

R. Massive, black tourmaline, Paris, Me.

S. Black tourmaline, Calhao, Brazil. Specific gravity, 3.20.

T. Black tourmaline, Haddam, Conn.

\begin{tabular}{|c|c|c|c|c|}
\hline & $Q$. & R. & s. & $\mathrm{T}$. \\
\hline $\mathrm{SiO}_{2} \ldots$ & 34.99 & 35.03 & 34.63 & 34.95 \\
\hline $\mathrm{TiO}_{2} \ldots$ & none & $\ldots$ & $\ldots .$. & .57 \\
\hline $\mathrm{Al}_{2} \mathrm{O}_{3}$ & 33.96 & 34.44 & 32.70 & 31.11 \\
\hline $\mathrm{Fe}_{2} \mathrm{O}_{3} \ldots \ldots$ & none & 1.13 & .31 & .50 \\
\hline $\mathrm{FeO} .$. & 14.23 & 12.10 & 13.69 & 11.87 \\
\hline $\mathrm{MnO} \ldots$ & .06 & .08 & .12 & .09 \\
\hline $\mathrm{MgO} \ldots$ & 1.01 & 1.81 & 2.13 & 4.45 \\
\hline $\mathrm{CaO}$ & .15 & .24 & .33 & .81 \\
\hline $\mathrm{Li}_{2} \mathrm{O} \ldots$ & trace & .07 & .08 & trace \\
\hline $\mathrm{Na}_{2} \mathrm{O} \ldots$ & 2.01 & 2.03 & 2.11 & 2.22 \\
\hline $\mathrm{K}_{2} \mathrm{O} \ldots \ldots \ldots$ & .34 & .25 & .24 & .24 \\
\hline $\mathrm{B}_{2} \mathrm{O}_{3}-$ & 9.63 & 9.02 & 9.63 & 9.92 \\
\hline $\mathrm{P}_{2} \mathrm{O}_{5} \ldots \ldots$ & trace & trace & none & trace \\
\hline $\mathrm{H}_{2} \mathrm{O}$ & 3.62 & 3.69 & 3.49 & 3.62 \\
\hline \multirow{2}{*}{ F } & none & none & .06 & none \\
\hline & 100.00 & 99.89 & 99.52 & 100.35 \\
\hline \multirow[t]{2}{*}{$\mathrm{O}$ equivalent to $\mathrm{F}$} & & & .02 & $\ldots .$. \\
\hline & & & 99.50 & $\cdots$ \\
\hline
\end{tabular}


U. Black crystal, Nantic Gulf, Baffin Land. Specific gravity, 3.095.

V. Black crystal, Stony Point, Alexander County, N. C. Specific gravity, 3.13 .

W. Brown tourinaline from near Colfax, Nevada County, Cal. Fine, radiating needles. Specific gravity, 3.065. Incomplete analysis by W. H. Melville. See Bulletin 90 .

X. Chrome tourmaline from near Etchison, Montgomery County, Md. Analysis by T. M. Chatard. See Bulletin 64 .

\begin{tabular}{|c|c|c|c|c|}
\hline & $\mathrm{U}$. & $\mathrm{v}$. & w. & $\mathrm{x}$. \\
\hline $\mathrm{SiO}_{2 .-}$ & 35.34 & 35.56 & 36.40 & 36.57 \\
\hline $\mathrm{TiO}_{2-}$ & .40 & .55 & $\ldots$ & .09 \\
\hline $\mathrm{Al}_{2} \mathrm{O}_{3}-$ & 30.49 & 33.38 & 33.64 & 32.58 \\
\hline $\mathrm{Cr}_{2} \mathrm{O}_{3}$ & $\cdots . .$. & - & - & 4.32 \\
\hline $\mathrm{Fe}_{2} \mathrm{O}_{3}$ & none & none & & \\
\hline $\mathrm{Fen} \ldots$ & 8.22 & 8.49 & 3.13 & 79 \\
\hline $\mathrm{MnO} \ldots$ & trace & .04 & . . n & trace \\
\hline $\mathrm{NiO}$ & .... & & $\ldots$ & .05 \\
\hline $\mathrm{MgO}$. & 7.76 & 5.44 & 10.01 & 9.47 \\
\hline $\mathrm{CaO} \ldots$ & 2.32 & .53 & 1.51 & .75 \\
\hline $\mathrm{SrO} \ldots$ & trace & none & $\ldots$ & \\
\hline $\mathrm{Li}_{2} \mathrm{O} \ldots .$. & trace & trace & 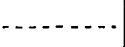 & trace \\
\hline $\mathrm{Na}_{2} \mathrm{O}_{-}$ & 1.76 & 2.16 & 2.49 & 2.22 \\
\hline $\mathrm{K}_{2} \mathrm{O} \ldots$ & .15 & .24 & .12 & .13 \\
\hline $\mathrm{B}_{2} \mathrm{O}_{3} \ldots \ldots$ & 10.45 , & 10.40 & a 8.74 & 8.90 \\
\hline $\mathrm{P}_{2} \mathrm{O}_{5} \ldots \ldots$ & none & (?) & $-\cdots$ & .04 \\
\hline $\mathrm{H}_{2} \mathrm{O} \ldots \ldots$ & 3.60 & 3.63 & 3.53 & 3.74 \\
\hline \multirow[t]{2}{*}{$\mathbf{F}$} & none & none & .74 & .06 \\
\hline & 100.49 & 100.42 & 100.31 & 99.71 \\
\hline \multirow[t]{2}{*}{ O equivalent to $\mathrm{F} \ldots \ldots \ldots \ldots$} & $\ldots$ & - ....... & .31 & .02 \\
\hline & & & 100.00 & 99.69 \\
\hline
\end{tabular}


GYROLITE.

Associated with the apophyllite of New Almaden, Cal. Fibrous. Analyzed by F. W. Clarke and described in Bulletin 64.

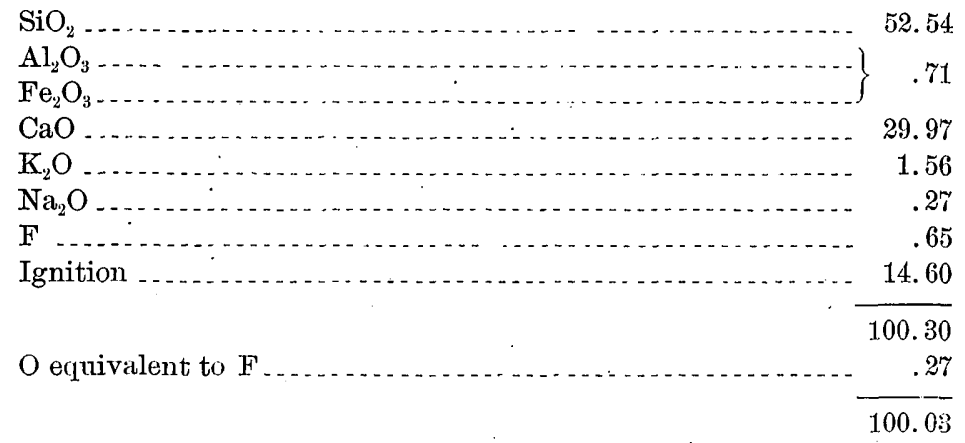

APOPHYLITTE.

From Table Mountain, Golden, Colo. Described by Cross and Hillebrand in Bulletin 20. Analyses by W. F. Hillebrand.

A. Apophyllite.

B. Decomposition product of apophyllite.

\begin{tabular}{|c|c|c|}
\hline . & A. & B. \\
\hline $\mathrm{SiO}_{2} \ldots \ldots \ldots$ & 51.89 & 67.96 \\
\hline $\mathrm{Al}_{2} \mathrm{O}_{3} \ldots$ & 1.54 & 8.48 \\
\hline $\mathrm{Fe}_{2} \mathrm{O}_{3}$ & .13 & 1.04 \\
\hline $\mathrm{CaO}, \ldots+\ldots$ & 24.51 & 5.47 \\
\hline $\mathrm{MgO} \ldots \ldots \ldots \ldots$ & (.......... & .53 \\
\hline $\mathrm{K}_{2} \mathrm{O} \ldots \ldots \ldots$ & 3.81 & 1.23 \\
\hline 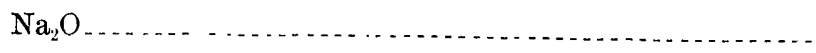 & .59 & a. 74 \\
\hline - & 16.52 & 14.55 \\
\hline ـ & 1.70 & none \\
\hline \multirow[t]{3}{*}{$\mathrm{O}$ equivalent to $\mathrm{F}$} & 100.69 & 100.00 \\
\hline & .72 & 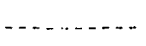 \\
\hline & 99.97 & $\ldots$ \\
\hline
\end{tabular}


P'TILOLITE.

Described as a new species by Cross and Eakins in Am. Jour. Sci., 3 d ser., vol. 32, p. 117, and vol. 44, p. 96 . Analyses by T. G. Eakins.

A. From Green Mountain, Jefferson County, Colo.

B. From 3 miles south of Silver Cliff; Custer County, Colo.

\begin{tabular}{|c|c|c|}
\hline & A. & B. \\
\hline $\mathrm{SiO}_{2} \ldots \ldots$ & 70.35 & 67.83 \\
\hline $\mathrm{Al}_{2} \mathrm{O}_{3} \ldots \ldots$ & 11.90 & 11.44 \\
\hline $\mathrm{CaO} \ldots \ldots$ & 3.87 & 3.30 \\
\hline $\mathrm{K}_{2} \mathrm{O} \ldots \ldots$ & 2.83 & .64 \\
\hline $\mathrm{Na}_{2} \mathrm{O}$ & .77 & 2.63 \\
\hline $\mathrm{H}_{2} \mathrm{O}$ at $110^{\circ} \ldots$ & \multirow{4}{*}{10.18} & 3.62 \\
\hline $\mathrm{H}_{2} \mathrm{O}$ at $125^{\circ} \ldots \ldots$ & & 1.31 \\
\hline $\mathrm{H}_{2} \mathrm{O}$ at $300^{\circ} \ldots \ldots$ & & 5.41 \\
\hline \multirow[t]{2}{*}{$\mathrm{H}_{2} \mathrm{O}$ at redness $\ldots \ldots \ldots$} & & 3.10 \\
\hline & $99.90^{\circ}$ & 99.28 \\
\hline
\end{tabular}

HEULANDITE.

A. From Green Mountain, Jefferson County, Colo. Analysis by L. G. Eakins.

B. From Anthracite Creek, Gunnison County, Colo. Analysis by L. G. Eakins. Specific gravity, 2.24 at 2() $.1^{\circ}$.

C. From Berufiörd, Iceland. Analysis by George Steiger.

\begin{tabular}{|c|c|c|c|}
\hline & A. & B. & C. \\
\hline $\mathrm{SiO}_{2} \ldots \ldots \ldots$ & 59.17 & 57.38 & 57.10 \\
\hline $\mathrm{Al}_{2} \mathrm{O}_{3} \ldots$ & 16.80 & 17.18 & 16.82 \\
\hline $\mathrm{Fe}_{2} \mathrm{O}_{3} \cdot \ldots \ldots \ldots$ & - & $\operatorname{trace}$ & $\ldots$ \\
\hline $\mathrm{MgO} \ldots \ldots .$. & 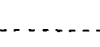 & $\ldots$ & .07 \\
\hline $\mathrm{CaO}_{-} \ldots \ldots$. & 7.10 & 8.07 & 6.95 \\
\hline $\mathrm{SrO} \ldots \ldots$ & $\ldots$ & $\cdots$ & .46 \\
\hline $\mathrm{Na}_{2} \mathrm{O}$ & 1.37 & .82 & 1.25 \\
\hline $\mathrm{K}_{2} \mathrm{O} \ldots \ldots \ldots$ & .34 & .40 & .42 \\
\hline $\mathrm{H}_{2} \mathrm{O}$ at $110^{\circ} \ldots$ & \multirow{4}{*}{15.45} & 2.57 & 3.61 \\
\hline $\mathrm{H}_{2} \mathrm{O}$ at $125^{\circ} \ldots$ & & 1.10 & \\
\hline $\mathrm{H}_{2} \mathrm{O}$ at $300^{\circ} \ldots \ldots \ldots$ & & 11.70 & 13.00 \\
\hline $\mathrm{H}_{2} \mathrm{O}$ at redness $\ldots \ldots \ldots$ & & .90 & \\
\hline & 100.23 & $100: 12$ & .99 .68 \\
\hline
\end{tabular}

Bull. 220-03-5 
STILBITE.

A. From Wassons Bluff, near Parrsboro, Nova Scotia. Analysis by George Steiger.

B. From Italian Mountain, Gunnison County, Colo. Analysis by L. G. Eakins.

C. From Table Mountain, Golden, Colo. Described by Cross and Hillebrand in Bulletin 20. Analysis by W. F. Hillebrand.

\begin{tabular}{|c|c|c|c|}
\hline & A. & B. & C. \\
\hline $\mathrm{SiO}_{2} \ldots \ldots$ & 55.41 & 57.75 & 54.67 \\
\hline $\mathrm{Al}_{2} \mathrm{O}_{3}$ & 16.85 & 16.64 & 16.78 \\
\hline $\mathrm{Fe}_{2} \mathrm{O}_{3} \ldots \ldots$ & .18 & & \\
\hline $\mathrm{MgO} \ldots \ldots . . . .$. & .05 & ..... & $\ldots$ \\
\hline $\mathrm{CaO}, \ldots . . .2$. & 7.78 & 8.58 & .7 .98 \\
\hline $\mathrm{Na}_{2} \mathrm{O}_{2}$ & 1.23 & trace & 1.47 \\
\hline $\mathrm{H}_{2} \mathrm{O}$ at $100^{\circ}$. & 3.60 & 1717 & 1916 \\
\hline \multirow[t]{2}{*}{$\mathrm{H}_{2} \mathrm{O}$ above $100^{\circ}$} & 15.41 & 18.18 & 19.10 \\
\hline & 100.51 & 100.14 & 100.06 \\
\hline
\end{tabular}

\section{LAUMONTITL.}

From Table Mountain, Golden, Colo. Described by Cross and Hillebrand in Bulletin 20. Analyses by W. F. Hillebrand.

A. Yellow grains.

B. White crystals.

\begin{tabular}{|c|c|c|}
\hline , & A. & B. \\
\hline (n) & 51.43 & 52.07 \\
\hline $\mathrm{Al}_{2} \mathrm{O}_{3} \ldots \ldots$ & 21.52 & 21.30 \\
\hline $\mathrm{Fe}_{2} \mathrm{O}_{3} \ldots$ & .94 & \\
\hline $\mathrm{CaO}$ & 11.88 & 11: 24 \\
\hline $\mathrm{K}_{2} \mathrm{O} \ldots \ldots$ & .35 & .42 \\
\hline $\mathrm{Na}_{2} \mathrm{O}$ & .19 & .48 \\
\hline \multirow{2}{*}{$\mathrm{H}_{2} \mathrm{O} \ldots \ldots$} & 13.81 & 14. 58 \\
\hline & 100.12 & 100.09 \\
\hline
\end{tabular}




\section{CHABAZITE.}

A. From Wassons Bluff, near Parrsboro, Nova Scotia. Analysis by George Steiger.

B, C: From Table Mountain, Golden, Colo. Described by Cross and Fillebrand in Bulletin 20. Analyses by W. F. Hillebrand.

\begin{tabular}{|c|c|c|c|}
\hline - & A. & I. & C. \\
\hline $\mathrm{SiO}_{2} \ldots \ldots \ldots$ & 50.78 & 47.86 & 47.18 \\
\hline $\mathrm{Al}_{2} \mathrm{O}_{3}$ & 17.18 & 19.30 & 19.67 \\
\hline $\mathrm{Fe}_{2} \mathrm{O}_{3} \ldots \ldots \ldots$ & .40 & .12 &.- \\
\hline $\mathrm{MgO}$ & .04 & $\ldots$ & $\ldots$ \\
\hline $\mathrm{CaO} \ldots . .$. & 7.84 & 9.94 & 9.74 \\
\hline $\mathrm{SrO} \ldots . .$. & ….... & & .43 \\
\hline $\mathrm{Na}_{2} \mathrm{O} \ldots$ & 1.28 & .52 & .51 \\
\hline $\mathrm{K}_{2} \mathrm{O} \ldots \ldots \ldots$ &. .73 & .35 & .37 \\
\hline $\mathrm{H}_{2} \mathrm{O}$ at $100^{\circ} \ldots$ & 5. 22 & 29 & 4.76 \\
\hline $\mathrm{H}_{2}^{*} \mathrm{O}$ above $100^{\circ} \ldots$ & 16.63 & 22.06 & 17.39 \\
\hline : & 100.10 & 100.16 & 100.05 \\
\hline
\end{tabular}

\section{LEVYNITE.}

From Table Mountain, Golden, Colo. Described by Cross and Hillebrand in Bulletin 20. Analyses by W. F. Hillebrand.

A. Crystals of levynite.

B. Associated fibrous variety.

\begin{tabular}{|c|c|c|}
\hline & A. & B. \\
\hline $\mathrm{SiO}_{2} \ldots \ldots \ldots$ & 46.76 & 46.97 \\
\hline $\mathrm{Al}_{2} \mathrm{O}_{3} \ldots \ldots$ & 21.91 & 22.39 \\
\hline $\mathrm{CaO}$ & 11.12 & 10.85 \\
\hline $\mathrm{K}_{2} \mathrm{O}_{2}$ & .21 & 1.17 \\
\hline $\mathrm{Na}_{4} \mathrm{O}$ & 1.34 & .79 \\
\hline \multirow{2}{*}{$\mathrm{H}_{2} \mathrm{O}$} & 18.65 & 18.03 \\
\hline & 99.99 & 100.20 \\
\hline
\end{tabular}




\section{ANALCI'LE.}

A. From Wassons Bluff, near Parrsboro, Nova Scotia. Analysis by George Steiger. . See Bulletin 207.

B. From Table Mountain, Golden, Colo. Analysis by George Steiger. See Bulletin 207.

C, D. From Table Mountain. Described by Cross and Hillebrand in Bulletin 20. Analyses by W. F. Hillebrand.

\begin{tabular}{|c|c|c|c|c|}
\hline & A. & B. & C. & $\mathrm{D}$. \\
\hline $\mathrm{SiO}_{2} \ldots$ & 57.06 & 55.72 & 55.82 & 55.80 \\
\hline $\mathrm{Al}_{2} \mathrm{O}_{3}$ & 21.48 & 23.06 & 22.42 & 22.45 \\
\hline $\mathrm{Fe}_{2} \mathrm{O}_{3}$ & .13 & & & - . . . . \\
\hline $\mathrm{CaO}$ & .16 & .17 & 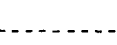 & - \\
\hline $\mathrm{Na}_{2} \mathrm{O}$ & 12.20 & 12.46 & 13.48 & 13.45 \\
\hline $\mathrm{H}_{2} \mathrm{O}$ at $100^{\circ}$ & .58 & .13 & 838 & 835 \\
\hline \multirow[t]{2}{*}{$\mathrm{H}_{2} \mathrm{O}$ above $100^{\circ} \ldots$} & 8.38 & 8.26 & 8.38 & \\
\hline & 99.99 & 99.80 & 100.10 & 100.05 \\
\hline
\end{tabular}

NATROLITE.

A. From Bergen Hill, New Jersey. Analysis by George Steiger. See Bulletin 207.

B. From Magnet Cove, Arkansas. Analysis by W. H. Melville. Described in Bulletin 90. Specific gravity, 2.261.

C. From South Table Mountain, Golden, Colo. Described by Cross and Hillebrand in Bulletin 20. Analysis by. W. F. Hillebrand:

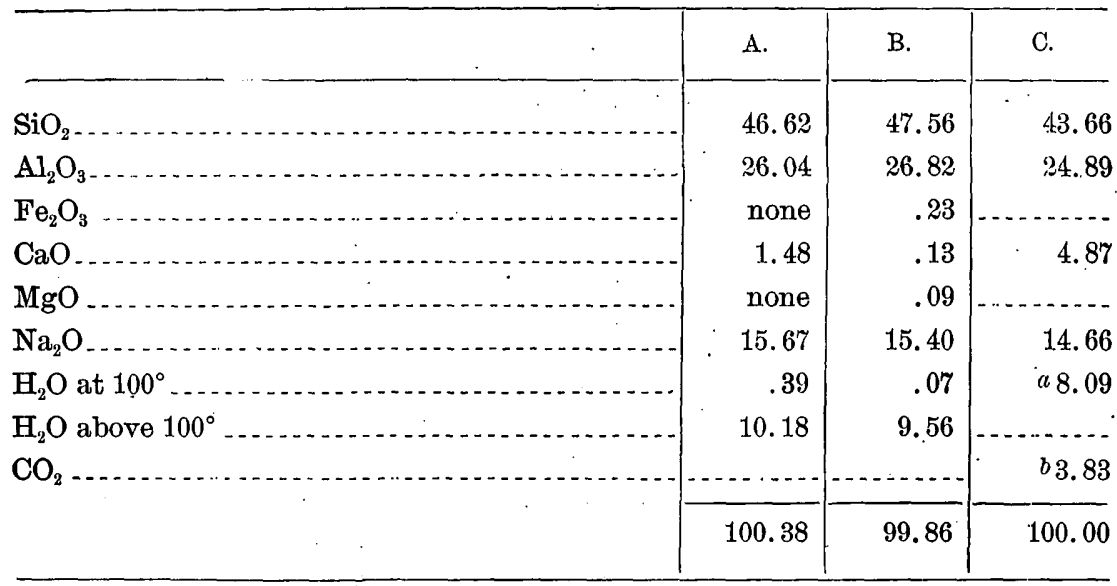

a By difference. Too little material for complete analysis.

$b$ Calculated to satisfy total line. 


\section{SCOLECITE.}

A. From Whale Cove, Grand Manan, New Brunswick. Analysis by George Steiger. See Bulletin 207.

B. From Italian Mountain, Gunnison County, Colo. Analysis by L. G. Eakins. Specific gravity, 2.247 at $17.2^{\circ}$.

C. From Table Mountain, Golden, Colo. Described by Cross and Hillebrand in Bulletin 20. Analysis by W. F. Hillebrand.

\begin{tabular}{|c|c|c|c|}
\hline & A. & B. & C. \\
\hline - & 45.86 & 45.90 & 46.03 \\
\hline $\mathrm{Al}_{2} \mathrm{O}_{3}$ & 25.78 & 26.51 & 25.28 \\
\hline $\mathrm{Fe}_{2} \mathrm{O}_{3}$ & & $\cdots$ & .27 \\
\hline $\mathrm{CaO}$ & 13.92 & 14.17 & 12.77 \\
\hline $\mathrm{MgO}_{\text {. }}$ & & trace & $\ldots$ \\
\hline $\mathrm{Na}_{2} \mathrm{O}$ & .41 & trace & 1.04 \\
\hline $\mathrm{K}_{2} \mathrm{O} \ldots$ & 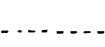 & 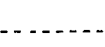 & .13 \\
\hline $\mathrm{H}_{2} \mathrm{O}$ at $100^{\circ} \ldots$ & .40 & $\ldots$ & 41448 \\
\hline \multirow[t]{2}{*}{$\mathrm{H}_{2} \mathrm{O}$ above $100^{\circ}$} & 13.65 & 13.79 & $a 14.40$ \\
\hline & 100.02 & 100.37 & 100.00 \\
\hline
\end{tabular}

$a$ By difference.

MESOLITE.

From North Table Mountain, Golden, Colo. $\because$ Described by Cross and Hillebrand in Bulletin 20. Analysis by W. F. Hillebrand.

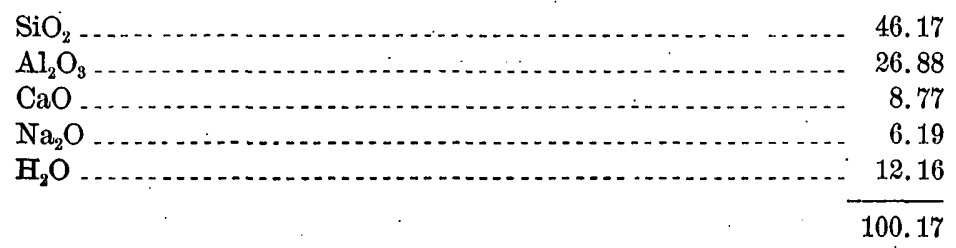




\section{THOMSONITE.}

From Table Mountain, Golden, Colo. Described by Cross and Hillebrand in Bulletin 20. Analyses A, B, C, D, E by W. F. Hillebrand. Analysis F by George Steiger; published in Bulletin 207. A represents reddish spherules; F, a mass of fibrous structure.

\begin{tabular}{|c|c|c|c|c|c|c|}
\hline . & A. & B. & C. & D. & E. & F. \\
\hline $\mathrm{SiO}_{2}$ & 40.52 & 40.88 & 40.68 & 41.21 & 42.66 & 41.13 \\
\hline $\mathrm{Al}_{2} \mathrm{O}_{3}$ & 29.22 & 29.68 & 30.12 & 29.71 & 29.25 & 29.58 \\
\hline $\mathrm{Fe}_{2} \mathrm{O}_{3} \ldots$ & .79 & & & & & \\
\hline $\mathrm{CaO}$ & 12.43 & 11.88 & 11: 92 & 11.34 & 10.90 & 11.25 \\
\hline \multirow{4}{*}{$\begin{array}{l}\mathrm{Na}_{2} \mathrm{O} \\
\mathrm{H}_{2} \mathrm{O} \text { at } 100^{\circ} \\
\mathrm{H}_{2} \mathrm{O} \text { above } 100^{\circ}\end{array}$} & 4.31 & 4. 72 & 4.44 & 5.62 & 4.92 & 5.31 \\
\hline & 1279 & (2) 91 & 1286 & 12 20 & 1228 & 1.01 \\
\hline & & (16.01 & $\int^{10.00}$ & $1 \sigma .20$ & $1+\alpha . \alpha 0$ & .12 .12 \\
\hline & 100.06 & 100.07 & 100.02 & 100.08 & 100.01 & 100.40 \\
\hline
\end{tabular}

\section{HYDRONEPHELITE.}

From the elæolite-syenite of Litchfield, Me. Described by Clarke as a new species in Bulletin 42. Analyses by F. W. Clarke. Specific gravity, 2.263, determined by J. S. Diller.

A, B. Slightly impure. Two distinct samples.

C. Carefully purified material dried at $100^{\circ}$.

\begin{tabular}{|c|c|c|c|}
\hline & A. & B. & c. \\
\hline $\mathrm{SiO}_{2} \ldots \ldots \ldots$ & 38.90 & 39.24 & 38.99 \\
\hline $\mathrm{Al}_{2} \mathrm{O}_{3} \ldots \ldots$ & 33.98 & 33.16 & 33.62 \\
\hline $\mathrm{Fe}_{2} \mathrm{O}_{3}$ & trace & trace & $-\cdots$ \\
\hline $\mathrm{CaO} \ldots \ldots$ & .05 & trace & .07 \\
\hline $\mathrm{Na}_{2} \mathrm{O}$ & 13.21 & 13.07 & 13.07 \\
\hline $\mathrm{K}_{2} \mathrm{O} \ldots \ldots$ & 1.01 & .88 & 1.12 \\
\hline \multirow[t]{2}{*}{$\mathrm{H}_{2} \mathrm{O}$} & 13.12 & 13.30 & 12.98 \\
\hline & 100.27 & 99.65 & 99.85 \\
\hline
\end{tabular}


MUSCOVITE.

A. From the Fatch farm, Auburn, Me. Broad plates, bordered by lepidolite. Analysis by R. B. Riggs.

13. Greenish muscovite, Auburn, Me. Analysis by E. A. Schneider.

C. Altered muscovite, Mount Mica, Paris, Me. Occurs as a white enamel on ordinary mica. Analysis hy F. W. Clarke.

D. Well-crystallized muscovite from the hiddenite mine, stony Point, Alexander County, N. C. Analysis by F. W. Clarke.

E. White muscovite from Miask, Ural Mountains, Siberia. Analysis by E. A. Schneider.

F. Mica separated from quartz-schist. Shoemaker's quarry, near Stevenson Station, Green Spring Valley, Maryland. Analysis by E. A. Schneider.

G. Mica from the Eureka tunnel, St. Peters Dome, near Pikes Peak, Colorado. Analysis by W. F. Hillebrand. See Bulletin 20.

\begin{tabular}{|c|c|c|c|c|c|c|c|}
\hline & A. & B. & C. & D. & E. & F. & G. \\
\hline $\mathrm{SiO}_{2}$ - & 44.39 & 46.54 & 46.61 & 45.40 & 44.17 & 44.93 & 52.59 \\
\hline $\mathrm{TiO}_{2} \ldots \ldots$ & $\ldots$ & none & - . . . & 1.10 & $\therefore \ldots$ & 1.05 & $\ldots \ldots$ \\
\hline $\mathrm{Al}_{2} \mathrm{O}_{3}$ & 35.70 & 34.96 & 35.61 & 33.66 & 37.35 & 29.81 & 29.72 \\
\hline $\mathrm{Fe}_{2} \mathrm{O}_{3} \ldots \ldots$ & 1.09 & 1.59 & & 2.36 & 1. 29 & 6.10 & 1.40 \\
\hline $\mathrm{FeO} \ldots \ldots . . . . .$. & 1.07 & 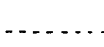 & & $\ldots$ & .20 & & $\cdot$ \\
\hline $\mathrm{MnO}$ & trace & $\ldots$ & trace & 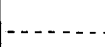 & .10 & trace & $\ldots$ \\
\hline $\mathrm{MgO}_{-}$ & ....... & .32 & D. & 1.86 & & 1.16 & 2.12 \\
\hline $\mathrm{CaO} \ldots$ & .10 & $\ldots$ & trace & - & & & .26 \\
\hline $\mathrm{Li}_{2} \mathrm{O}_{-}$ & $\ldots$ & $\ldots$ & 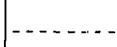 & trace & $\cdots \ldots$ & trace & $\ldots$ \\
\hline $\mathrm{Na}_{2} \mathrm{O}_{-}$ & 2.41 & .41 & 1.76 & 1.41 & 1.14 & .50 &. .50 \\
\hline $\mathrm{K}_{2} \mathrm{O} \ldots \ldots$ & 9.77 & 10.38 & 8.86 & 8.33 & 10.00 & $10.28^{\circ}$ & 8.33 \\
\hline $\mathrm{H}_{2} \mathrm{O}$ at $100^{\circ}$. & 580 & .71 & 650 & $16 \quad$ & 1.06 & 1.38 & 20 \\
\hline $\mathrm{H}_{2} \mathrm{O}$ above $100^{\circ}$. & 0.88 & 4.72 & 6.50 & 5.46 & 4.67 & 4.88 & 4.39 \\
\hline \multirow[t]{2}{*}{ F } & .72 & none & & .69 & .90 & .22 & \\
\hline & 101.13 & - 99.63 & 99.34 & 100.27 & 100.88 & 100.31 & 99.31 \\
\hline \multirow[t]{2}{*}{ O equivalent to F } & .30 & &.- & .29 & .37 & .09 & $\ldots$ \\
\hline & 100.83 & & -. & 99.98 & 100.51 & 100.22 & \\
\hline
\end{tabular}


H. Fuchsite. Etchison post-office, Montgomery County, Md. Analysis by T. M. Chatard. See Bulletin 64.

I. Grayish-green, compact mica from Stoneham, Me. Structure subfibrous. Analysis by T. M. Chatard.

J. Same locality as I. Specimen broadly foliated, micaceous. Analysis by T. M. Chatard. For analyses I and $J$ see Bulletin 9.

K. Stoneham, Me. From alteration of topaz, the outer portion of an altered crystal. Specific gravity, 2.82. Analysis by J. E. Whitfield. Discussed by Clarke in Bulletin 27.

L. Pseudomorph from the Rochelle mine, on Running Water: River, Wyoming. Near liebenerite. Specific gravity, 2.831 at $12.5^{\circ}$. Analyzed by W. F. Hillebrand and described in Bulletin 20.

M. Mariposite. From vein of the Josephine gold quartz mine, Bear Valley, Mariposa County, Cal. Color, green. Specific gravity, 2.817 at $29.5^{\circ}$.

$\mathrm{N}$. White mariposite, saine locality as M. Specific gravity, 2.787 at $28.5^{\circ}$. Analyses $\mathrm{M}$ and $\mathrm{N}$ by W. F. Hillebrand, and described in Bulletin 167.

\begin{tabular}{|c|c|c|c|c|c|c|c|}
\hline & H. & I. & J. & $\mathrm{K}$. & L. & M. & N. \\
\hline $\mathrm{SiO}_{2-}$ & \multirow[t]{2}{*}{42.21} & \multirow[t]{2}{*}{45.19} & \multirow[t]{2}{*}{45.34} & \multirow[t]{2}{*}{44.52} & \multirow[t]{2}{*}{45.54} & \multirow{2}{*}{$\begin{array}{r}55.35 \\
.18\end{array}$} & \multirow[t]{2}{*}{56.79} \\
\hline $\mathrm{TiO}_{2} \ldots$ & & & & & & & \\
\hline $\mathrm{Al}_{2} \mathrm{O}_{3}$ & \multirow{2}{*}{$\begin{array}{r}34.55 \\
2.03\end{array}$} & 33.32 & 33.96 & 46.19 & 37.15 & $\begin{array}{r}25.62 \\
18\end{array}$ & \multirow{2}{*}{$\begin{array}{l}25.29 \\
\text { none }\end{array}$} \\
\hline $\mathrm{Cr}_{2} \mathrm{O}_{3}$ & & & & 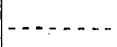 & .79 & & \\
\hline $\mathrm{Fe}_{2} \mathrm{O}_{3} \ldots$ & \multirow[t]{2}{*}{1.03} & $\ldots$ & $\cdots$ & & & .63 & \multirow[t]{3}{*}{1.59} \\
\hline $\mathrm{FeO} \ldots$ & & 4.25 & 3.96 & $\ldots$ & & \multirow[t]{2}{*}{.92} & \\
\hline $\mathrm{MnO}$ & \multirow{2}{*}{$\begin{array}{r}\text { trace } \\
47\end{array}$} & .58 & .51 & .21 & & & \\
\hline $\mathrm{CaO}$ & & trace & .22 & .30 & -. & .07 & .07 \\
\hline $\mathrm{MgO}$. & \multirow[t]{2}{*}{3.13} & \multirow[t]{2}{*}{.36} & \multirow[t]{2}{*}{.10} & \multirow[t]{2}{*}{.14} & \multirow[t]{2}{*}{.38} & \multirow{2}{*}{$\begin{array}{r}3.25 \\
\text { trace }\end{array}$} & \multirow{2}{*}{$\begin{array}{r}3.29 \\
\text { trace }\end{array}$} \\
\hline $\mathrm{Li}_{2} \mathrm{O}_{\ldots} \ldots$ & & & & & & & \\
\hline $\mathrm{Na}_{2} \mathrm{O} \ldots$ & .82 & \multirow{2}{*}{$\begin{array}{r}1.57 \\
11.06\end{array}$} & \multirow{2}{*}{$\begin{array}{r}1.49 \\
10.73\end{array}$} & \multirow{2}{*}{$\begin{array}{l}2.82 \\
2.30\end{array}$} & .90 & .12 & .17 \\
\hline $\mathrm{K}_{2} \mathrm{O} \ldots \ldots$ & $\begin{array}{r}9.16 \\
20\end{array}$ & & & & 10.70 & 9.29 & 3.92 \\
\hline $\mathrm{H}_{2} \mathrm{O}$ at $110^{\circ}$.. & .20 & \multirow{3}{*}{4.48} & \multirow{3}{*}{4.78} & 3.74 & 80 & 4.52 & 472 \\
\hline $\mathrm{H}_{2} \mathrm{O}$ above $110^{\circ}$. & 6.57 & & & & & & T. \\
\hline$F$ & $\cos \theta$ & & & .40 & & $\cdots$ & \\
\hline & 100.17 & 100.81 & 101.09 & 100.62 & 100.26 & 100.13 & 100.84 \\
\hline $\mathrm{O}$ equivalent to $\mathrm{F}$ & $2-2$ & ...... & $-\ldots, \ldots$ & .16 & $\ldots$ & $-\cdots$ & $\ldots \ldots$ \\
\hline & & & & 100.46 & & & \\
\hline
\end{tabular}


I.EPIDOLITE.

From various localities in Maine. Analyses by R. B. Riggs. Discussion by F. W. Clarke in Bulletin 42.

A. From Black Mountain, Rumford. Lilac-purple, granular.

B. From Mount Mica, Paris. Broadly foliated.

C. From Hebron. Purple, coarsely granular.

D. From Auburn. Purple border on plates of muscovite.

E. From Auburn. Purple, coarsely granular.

F. From Norway. White, coarsely granular.

G. From Norway. Brownish, finely granular.

\begin{tabular}{|c|c|c|c|c|c|c|c|}
\hline & A. & B. & C. & D. & E. & F. & G. \\
\hline $\mathrm{SiO}_{2-}$ & 51.52 & 50.92 & 48.80 & 49.62 & 51.11 & 49.52 & 50.17 \\
\hline $\mathrm{Al}_{2} \mathrm{O}_{3}$ & 25.96 & 24.99 & 28.30 & 27.30 & 25.26 & 28.80 & 25.40 \\
\hline $\mathrm{Fe}_{2} \mathrm{O}_{3}$ & .31 & .30 & .29 & .31 & .20 & .40 & .87 \\
\hline $\mathrm{FeO}$ & undet. & .23 & .09 & .07 &, .07 & .24 & .45 \\
\hline $\mathrm{MnO}$ & .20 & trace & .08 & .55 & .17 & .07 & .23 \\
\hline $\mathrm{CaO}$ & .16 & trace & .10 & $\ldots$ & .12 & .13 & undet. \\
\hline $\mathrm{MgO}$ & .02 & trace & $\cdot .07$ & $\ldots$ & .01 & .02 & undet. \\
\hline $\mathrm{Li}_{2} \mathrm{O}-$ & 4.90 & 4.20 & 4.49 & 4. 34 & 4.98 & 3.87 & 4.03 \\
\hline $\mathrm{Na}_{2} \mathrm{O}_{-}$ & 1.06 & 2.11 & .74 & 2.17 & 1.43 & .13 & \\
\hline $\mathrm{K}_{2} \mathrm{O} \ldots$ & 11.01 & 11.38 & 12.21 & 8.03 & 10.51 & 8.82 & 13.40 \\
\hline $\mathrm{Rb}_{2} \mathrm{O}$ & $\therefore$ & trace & & 2.44 & 1.29 & 3.73 & \\
\hline $\mathrm{Cs}_{2} \mathrm{O}$ & $\cdots$ & $\operatorname{trace}$ & . & .72 & .45 & .08 & \\
\hline $\mathrm{H}_{2} \mathrm{O}$ & .95 & 1.96 & 1.73 & 1.52 & .94 & 1.72 & 2.02 \\
\hline \multirow[t]{2}{*}{$\mathbf{F}$} & 5.80 & 6.29 & 4.96 & 5.45 & 6.57 & 5.18 & 5.05 \\
\hline & 101.89 & 102.38 & 101.86 & 102.52 & 103.11 & 102.71 & 101.62 \\
\hline \multirow[t]{2}{*}{ O equivalent to $\mathrm{F}$. } & 2.44 & 2.64 & 2.02 & 2.29 & 2.76 & 2.18 & 2.13 \\
\hline & 99.45 & 99.74 & 99.84 & 100.23 & 100.35 & 100.53 & 99.49 \\
\hline
\end{tabular}




\section{CRYOPHYLLITE.}

From Rockport, Mass. Analyses by R. B. Riggs. Discussed by Clarke in Bulletin 42.

A. Brilliant, broadly foliated, blackish-green variety.

B. Paler, dull green, less lustrous, probably somewhat altered.

C. Granular, resembling chlorite.

\begin{tabular}{|c|c|c|c|}
\hline . & A. & B. & C. \\
\hline $\mathrm{SiO}_{2-}$ & 51.96 & 51.46 & 52.17 \\
\hline $\mathrm{Al}_{2} \mathrm{O}_{3} \ldots$ & 16.89 & 16.22 & 16.39 \\
\hline $\mathrm{Fe}_{2} \mathrm{O}_{3}$ & 2.63 & 2.21 & 4.11 \\
\hline $\mathrm{FeO}$ & 6.35 & 7.66 & 6:08 \\
\hline $\mathrm{MnO} \ldots$ & .24 & .06 & .32 \\
\hline $\mathrm{CaO} \ldots$ & .12 & trace & trace \\
\hline $\mathrm{MgO}$ & .03 & .17 & tracë \\
\hline $\mathrm{Li}_{2} \mathrm{O}$ & 4.93 & 4.83 & 5.03 \\
\hline $\mathrm{Na}_{2} \mathrm{O} \ldots$ & .92 & .95 & .60 \\
\hline $\mathrm{K}_{2} \mathrm{O} \ldots \ldots \ldots$ & 10.66 & 10.65 & 10.54 \\
\hline $\mathrm{H}_{2} \mathrm{O} \ldots$ & 1.22 & 1.06 & 1.43 \\
\hline \multirow[t]{2}{*}{$\mathbf{F}$} & 6.78 & 7.44 & 7.02 \\
\hline & 102.73 & 102.71 & 103.69 \\
\hline \multirow{2}{*}{ O equivalent to $\mathrm{F}$} & 2.86 & 3.11 & $2: 95$ \\
\hline & 99.87 & 99.60 & 100.74 \\
\hline
\end{tabular}

BIOTITE.

A. From Merrow Ledge, Auburn, Me. Black. Analysis by F. W. Clarke and R. B. Riggs.

B. Bronze-mica, Laurel Creek corundum mine, Rabun County, Ga. Analysis by $\mathrm{E}$. A. Schneider. The loss is due to undetermined volatile matter.

C. Brown mica from the granite of the Yosemite Valley, California. Analysis by William Valentine. Described by'Turner, Am. Jour. Sci., 4th ser., vol. 7, p. 294.

D. Mica from quartz-monzonite, Tioga road, southeast of Mount Hoffman, Mariposa County, Cal. Specific gravity, 3.05 at 21‥ Analysis by W. F. Hillebrand. Described by Turner, loc. cit. 
E. Black mica from quartz-monzonite. About 1 kilometer south of Bloods Station, Alpine County, Cal. Analysis by William Valentine. Described by Turner, loc. cit.

F. Reddish-brown mica from pyroxenic gneiss. North fork of Mokelumne River, above mouth of Bear River, Amador County; Cal. Analysis by William Valentine. Described by Turner, loc. cit.

G. Biotite from the granite of Butte, Mont. See Weed, Jour. Geol., vol. 7, p. 737. Analysis by H. N. Stokes.

\begin{tabular}{|c|c|c|c|c|c|c|c|}
\hline & A. & B. & c. & D. & E. & w. & G. \\
\hline $\mathrm{SiO}_{2-}$ & 34.67 & 36.12 & 35.64 & 35.75 & 35.62 & 36.62 & 35.79 \\
\hline $\mathrm{TiO}_{2}$ & - . & 1.18 & 1.12 & 3.16 & 2.61 & 3.03 & 3.51 \\
\hline $\mathrm{Al}_{2} \mathrm{O}_{3}$ & 30.09 & 20.49 & 18.62 & 14.70 & 15.24 & 14.37 & 13.70 \\
\hline $\mathrm{V}_{2} \mathrm{O}_{3}$ & & . & & .05 & . & & \\
\hline \multicolumn{8}{|l|}{$\mathrm{Cr}_{2} \mathrm{O}_{3} \ldots \ldots \ldots \ldots$} \\
\hline $\mathrm{Fe}_{2} \mathrm{O}_{3}$ & 2.42 & 3.29 & 5.54 & 4.65 & 4.69 & 4.04 & 5.22 \\
\hline $\mathrm{FeO}$ & 16.14 & 5.17 & 14.60 & 14.08 & 13.67 & 17.09 & 13.72 \\
\hline $\mathrm{MnO} \ldots$ & .85 & .17 & .79 & .45 & .74 & .40 & .19 \\
\hline $\mathrm{NiO}$ & & .34 & & & & & \\
\hline \multicolumn{8}{|l|}{$\mathrm{CoO}$} \\
\hline $\mathrm{CaO}_{\text {.. }}$ & \multirow{2}{*}{ none } & \multirow[t]{2}{*}{.28} & \multirow[t]{2}{*}{.90} & .17 & .95 & 1.48 & .05 \\
\hline $\mathrm{SrO}$ & & & & (?) & trace & trace & \\
\hline $\mathrm{BaO}$ & $\ldots$ & .09 & trace & .12 & .26 & .33 & .13 \\
\hline $\mathrm{MgO}_{\text {. }}$ & \multirow{2}{*}{1.98} & \multirow{2}{*}{$\begin{array}{l}19.61 \\
\operatorname{trac} \theta\end{array}$} & \multirow{2}{*}{$\begin{array}{r}9.72 \\
\text { trace }\end{array}$} & \multirow{2}{*}{12.37} & \multirow{2}{*}{$\begin{array}{l}12.70 \\
\text { trace }\end{array}$} & \multirow{2}{*}{$\begin{array}{r}9.68 \\
\text { trace }\end{array}$} & \multirow{2}{*}{$\begin{array}{l}12.13 \\
\text { trace }\end{array}$} \\
\hline $\mathrm{Li}_{2} \mathrm{O}$ & & & & & & & \\
\hline $\mathrm{Na}_{42} \mathrm{O} \ldots$ & 1.67 & .78 & .38 & .32 & .50 & .45 & .15 \\
\hline $\mathrm{K}_{2} \mathrm{O} \ldots$ & 7.55 & 8.14 & 9.22 & 9.19 & 7.72 & 8.20 & 9.09 \\
\hline $\mathrm{H}_{2} \mathrm{O}$ at $105^{\circ}$ & \multirow{3}{*}{4.64} & \multirow{3}{*}{$\begin{array}{r}1.58 \\
.82\end{array}$} & \multirow{2}{*}{$\begin{array}{r}.48 \\
2.54\end{array}$} & 1.03 & .94 & .90 & 1.21 \\
\hline $\mathrm{H}_{2} \mathrm{O}$ above $105^{\circ}$ - & & & & 3.64 & 4.36 & 3.26 & 3.64 \\
\hline $\mathrm{P}_{2} \mathrm{O}_{5}$ & & & .20 & .03 & none & none & .10 \\
\hline F ... & \multirow[t]{2}{*}{.28} & \multirow[t]{2}{*}{.60} & \multirow[t]{2}{*}{.26} & \multirow[t]{2}{*}{.17} & \multirow[t]{2}{*}{ none } & \multirow[t]{2}{*}{.10} & .76 \\
\hline \multirow[t]{2}{*}{$\mathrm{Cl}_{-}$} & & & & & & & .20 \\
\hline & 100.29 & 98.66 & 100.01 & 99.90 & 100.00 & 99.95 & 99.59 \\
\hline \multirow[t]{2}{*}{ Less 0} & .12 & .26 & .11 & .07 & -- & .04 & .37 \\
\hline & 100.17 & 98.40 & 99.90 & 99.83 & & 99.91 & 99.22 \\
\hline
\end{tabular}




\section{PHLOGOPITE.}

A. From Edwards, St. Lawrence County, N.Y. Outwardly resembles talc. Analysis by E. A. Schneider. Discussed by Clarke and Schneider in Brlletin 78.

B. From Burgess, Canada. Brown. Analysis by E. A. Schneider. See Clarke and Schneider, Bulletin 78.

C. Phlogopite separated from the wyomingite of the Tencite Hills, Wyoming. Described by Cross in Am. Jour. Sci., 4th ser., vol. 4; p. 115. Analysis by W. F. Hillebrand.

The following micas of doubtful character occur in serpentine. Analyses by Charles Catlett. See Bulletin 64 .

D. Brown mica, from the serpentine of Montville, N. J.

E. Yellowish mica, same locality as D.

F. White mica, same locality.

G. White mica from near Easton, Pa.

E, F, and $\mathrm{G}$ are perhaps to be called vermiculites rather than micas.

\begin{tabular}{|c|c|c|c|c|c|c|c|}
\hline & A. & B. & c. & D. & E. & F. & G. \\
\hline $\mathrm{SiO}_{2} \ldots$ & 45.05 & 39.66 & 42.56 & 39.38 & 32.52 & 39.14 & 41.82 \\
\hline $\mathrm{TiO}_{2} \ldots \ldots$ & . & .56 & 2.09 & $\cdots$ & $\cdots$ & $-\therefore-1$ & $\ldots$. \\
\hline $\mathrm{Al}_{2} \mathrm{O}_{3} \ldots \ldots$ & 11.25 & 17.00 & 12.18 & 15.92 & 18.14 & 15.70 & 11. 12 \\
\hline $\mathrm{Cr}_{2} \mathrm{O}_{3} \ldots \ldots$ & $\ldots-$ & ….. & .73 & $\ldots$ & $\ldots$ & $\cdots$ & $\ldots .$. \\
\hline $\mathrm{Fe}_{2} \mathrm{O}_{3} \ldots$ & $\ldots$ & .27 & 2.73 & .71 & 2.30 & 1.68 & 2.68 \\
\hline $\mathrm{FeO} \ldots$ & .14 & .20 & .90 & .80 & & & \\
\hline $\mathrm{CaO} \ldots$ & 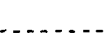 & none & .20 & . . 28 & 1.44 & 5.24 & $\ldots$ \\
\hline $\mathrm{SrO}$ & & $\ldots$ & trace & & -2 & $\ldots$ & 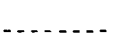 \\
\hline $\mathrm{BaO}$ & & .62 & 1.00 & & & & $\ldots$ \\
\hline $\mathrm{MgO}$. & 29.38 & 26.49 & 22.40 & 26.85 & 29.26 & 25.44 & 29.82 \\
\hline $\mathrm{Li}_{2} \mathrm{O} \ldots$ & .07 & ..... & trace & & & & $\cdots$ \\
\hline $\mathrm{Na}_{2} \mathrm{O} \ldots \ldots . . .$. & .45 & .60 & .44 & .62 & 1.38 & .64 & .36 \\
\hline $\mathrm{K}_{2} \mathrm{O} \ldots \ldots$ & .8 .52 & 9.97 & 10.70 & 9.84 & 2.78 & 2.06 & 6.08 \\
\hline $\mathrm{H}_{2} \mathrm{O}$ at $105^{\circ} \ldots$ & \multirow{3}{*}{5.37} & .66 & - . . & .38 & .76 & i. 10 & .94 \\
\hline $\mathrm{H}_{2} \mathrm{O}$ above $105^{\circ}$. & & 2.33 & 2.35 & 4.69 & 10.12 & 9.10 & 7.10 \\
\hline $\mathrm{CO}_{2} \ldots$ & & 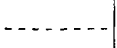 & $\ldots . .$. & .30 & 1.80 & 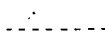 & $\ldots$ \\
\hline $\mathrm{P}_{2} \mathrm{O}_{5} \ldots$ & & trace & .06 & & & & \\
\hline \multirow[t]{2}{*}{$F$} & $\cdots$ & 2.24 & 2.46 & .62 & - & & - \\
\hline & 100.23 & 100.60 & 100.80 & 100.39 & 100.50 & 100.10 & 99.92 \\
\hline \multirow[t]{2}{*}{$\mathrm{O}$ equivalent to $\mathrm{F}$. } & & .94 & 1.03 & .26 . & & & -- \\
\hline & & $99.66-$ & 99.77 & 100.13 & & & \\
\hline
\end{tabular}




\section{LEPIDOMELANE.}

A. From Litchfield, Me. Analyzed by F. W. Clarke, and described in Bulletin 42 .

B. Same as A. Later analysis by Clarke. See Bulletin 55.

C. Annite. From Rockport, Mass. Analysis by R. B. Riggs. Discussion by Clarke in Bulletin 42.

D. From Port Henry, N.'Y. Analysis by E. A. Schneider. See paper by Clarke and Schneider in Bulletin 78 .

E. From Baltimore, Md. Analysis by F. W. Clarke, and discussed . in Bulletin 55.

F. From near Pikes Peak, Colorado. Siderophyllite? The slightly altered margin of a large crystal.

G. Much altered central portion of specimen F. Analyses F and G by F. W. Clarke and R. B. Riggs. Described by Clarke in Bulletin 55. Classed by D́ana under biotite.

\begin{tabular}{|c|c|c|c|c|c|c|c|}
\hline & A. & B. & C. & D. & E. & F. & G. \\
\hline $\mathrm{SiO}_{2}$ & \multirow[t]{2}{*}{32.09} & \multirow{2}{*}{$\begin{array}{l}32.35 \\
\text { trace }\end{array}$} & \multirow{2}{*}{$\begin{array}{r}32.03 \\
3.42\end{array}$} & \multirow{2}{*}{$\begin{array}{r}34.52 \\
2.70\end{array}$} & \multirow[t]{2}{*}{35.78} & \multirow[t]{2}{*}{34.21} & \multirow{2}{*}{$\begin{array}{r}34.63 \\
-\ldots . . .\end{array}$} \\
\hline $\mathrm{TiO}_{2} \ldots$ & & & & & & & \\
\hline $\mathrm{Al}_{2} \mathrm{O}_{3}$ & 18.52 & 17.47 & 11.92 & 13.22 & 16.39 & 16.53 & 17.95 \\
\hline $\mathrm{Fe}_{2} \mathrm{O}_{3} \ldots \ldots$ & 19.49 & 24.22 & 8.00 & 7.80 & 14.55 & 20.15 & 31.25 \\
\hline $\mathrm{FeO}$ & 14.10 & 13.11 & 30.41 & 22.27 & 11.02 & 14.17 & 3.01 \\
\hline $\mathrm{MnO}$ & \multirow[t]{2}{*}{$1: 42$} & \multirow{2}{*}{$\begin{array}{r}1.02 \\
\text { none }\end{array}$} & \multirow{2}{*}{$\begin{array}{l}.21 \\
.23\end{array}$} & .41 & 1.08 & .91 & .34 \\
\hline $\mathrm{CaO}_{-}$ & & & & none & none & .48 & .81 \\
\hline $\mathrm{MgO}$ & \multirow[t]{2}{*}{1.01} & \multirow[t]{2}{*}{.89} & \multirow[t]{2}{*}{.06} & 5.82 & \multirow[t]{2}{*}{8.67} & 1.34 & \multirow[t]{2}{*}{1.08} \\
\hline $\mathrm{NiO}, \mathrm{CoO}$ & & & & .30 & & & \\
\hline $\mathrm{Li}_{2} \mathrm{O}$ & $\cdots$ & $\cdots$ & trace & .04 & $\ldots$ & $\ldots$ & $\ldots$ \\
\hline $\mathrm{Na}_{2} \mathrm{O}$ & 1.55 & .70 & 1.54 & .16 & .56 & 1.43 & .89 \\
\hline $\mathrm{K}_{2} \mathrm{O} \ldots$ & 8.12 & 6.40 & 8.46 & 8.59 & 7.76 & 6.50 & 1.96 \\
\hline $\mathrm{H}_{2} \mathrm{O}$ at $105^{\circ} \ldots$ & \multirow{2}{*}{4.62} & \multirow{3}{*}{4.67} & \multirow{3}{*}{4.19} & .57 & \multirow{3}{*}{4.48} & \multirow{3}{*}{4.54} & \multirow{2}{*}{7.82} \\
\hline $\mathrm{H}_{2} \mathrm{O}$ above $105^{\circ}$. & & & & 3.82 & & & \\
\hline $\mathrm{P}_{2} \mathrm{O}_{5} \ldots \ldots$ & & & & trace & & & \\
\hline \multirow[t]{2}{*}{$\mathbf{F} \ldots$} & none & none & trace & .34 & none & .08 & .54 \\
\hline & 100.92 & 100.83 & 100.47 & 100.56 & 100.29 & 100.34 & 100.28 \\
\hline \multirow[t]{2}{*}{ O equivalent to $F_{.}$. } & & 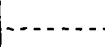 & - & .14 & $-\cdots$ & .03 & .22 \\
\hline & & & & 100.42 & & 100.31 & 100.06 \\
\hline
\end{tabular}


ROSCOELITE.

Analyses by W. F. Fillebrand.

A. From the Stockslager mine, near Lotus, Eldorado County, Cal. Described by Hillebrand in Bulletin 167.

B. Soluble portion of a vanadiferous sandstone from Placerville, Colo. Described by Hillebrand in Am. Jour. Sci., 4th ser., vol. 10, p. 130 .

\begin{tabular}{|c|c|c|}
\hline & A. & B. \\
\hline $\mathrm{SiO}_{2-}$ & 45.17 & 46.06 \\
\hline $\mathrm{TiO}_{2}$ & .78 & ...... \\
\hline $\mathrm{Al}_{2} \mathrm{O}_{3}$ & 11.54 & 22.55 \\
\hline $\mathrm{V}_{2} \mathrm{O}_{3} \ldots$ & 24.01 & 12.84 \\
\hline $\mathrm{Fe}_{2} \mathrm{O}_{3} \ldots \ldots \ldots$ & $\ldots \ldots$ & .73 \\
\hline $\mathrm{FeO} \ldots \ldots$ & 1.60 & ....... \\
\hline $\mathrm{CaO} \ldots \ldots . . . . . . . .$. & $\ldots . .$. & .44 \\
\hline $\mathrm{BaO}_{\ldots} \ldots \ldots$ & 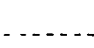 & 1.35 \\
\hline $\mathrm{MgO} \ldots . .$. & 1.64 & .92 \\
\hline $\mathrm{Li}_{2} \mathrm{O} \ldots \ldots$ & trace & - - - \\
\hline $\mathrm{Na}_{2} \mathrm{O}$ & trace & .22 \\
\hline $\mathrm{K}_{2} \mathrm{O}$ & 10.37 & 8.84 \\
\hline $\mathrm{H}_{2} \mathrm{O}$ at $100^{\circ}$ & .40 & 1.98 \\
\hline \multirow[t]{2}{*}{$\mathrm{H}_{2} \mathrm{O}$ above $100^{\circ}$} & 4.29 & 4.07 \\
\hline & 99.80 & 100.00 \\
\hline
\end{tabular}

\section{MARGARITE.}

A. Brownish yellow, from Iredell County, N. C. Analysis by 'T. M. Chatard. See Bulletin 9.

B. Bright green, associated with pink corundum. From Gainesville, Ga. Specific gravity, 3.00. Analysis by T. M. Chatard. See Bulletin 9.

C. Inclusion in diorite, Crugers Station, near Peekskill, N. Y. Analysis by T. M. Chatard.

\begin{tabular}{|c|c|c|c|}
\hline & A. & B. & $\dot{\mathrm{c}}$. \\
\hline $\mathrm{SiO}_{2} \ldots \ldots$ & 31.15 & 31.72 & 32.73 \\
\hline $\mathrm{Al}_{2} \mathrm{O}_{3}$ & 49.51 & 50.03 & 46.58 \\
\hline $\mathrm{FeO}_{\ldots} \ldots \ldots$ & trace & $\operatorname{trace}$ & 5.12 \\
\hline $\mathrm{MgO} \ldots \ldots$ & .45 & .12 & 1.00 \\
\hline $\mathrm{CaO} \ldots \ldots$ & 11.13 & 11.57 & 11.04 \\
\hline $\mathrm{Na}_{2} \mathrm{O}$ & 2.74 & 2.26 & \\
\hline \multirow{3}{*}{$\begin{array}{l}\mathrm{H}_{2} \mathrm{O} \text { at } 110^{\circ} \\
\mathrm{H}_{2} \mathrm{O} \text { above } 110^{\circ}\end{array}$} & 5. 6 & 4.88 & .12 \\
\hline & & & \\
\hline & 100.66 & 100.58 & 101.08 \\
\hline
\end{tabular}


CHLORITOID.

From a phyllite rock near Liberty, Ma. Analysis by I. G. Eakins. See Bulletin 168, p. 50.

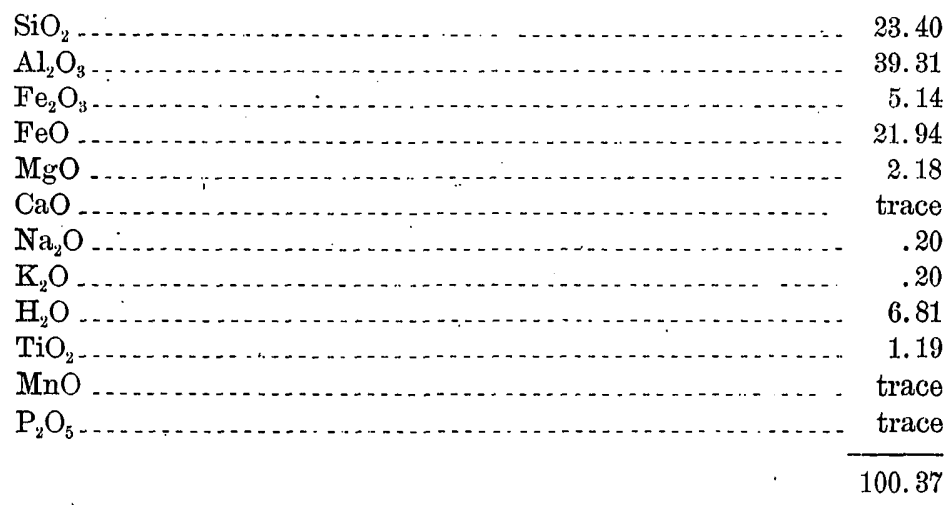

XANTHOPHYLLITE.

Variety waluewite, from the Nikolai-Maximilian mine, district of Slatoust, Urals, Siberia. Analysis by E. A. Schneider. Discussion by Clarke and Schneider in Bulletin 113.

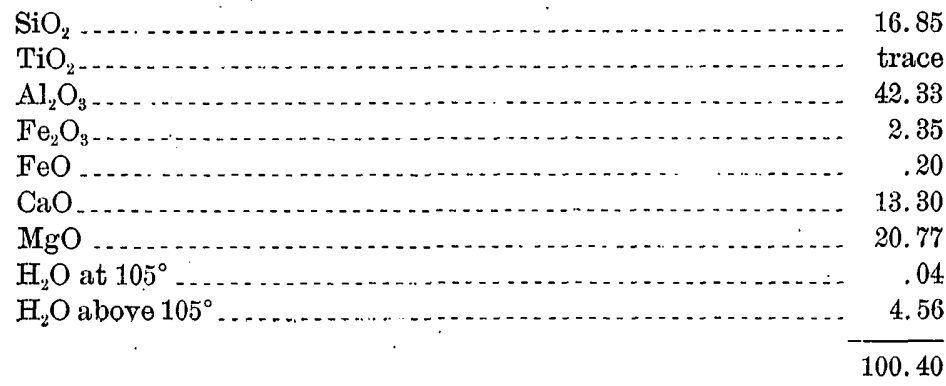


THE VERMICULITES.

A. Jefferisite, from Westchester, Pa. Analysis by E. A. Schneider. Discussed by Clarke and Schneider in Bulletin 78.

B. Vermiculite, near jefferisite or culsageeite, from Corundum Hill, North Carolina. Analyzed by T. M. Chatard and described by him.in Bulletin 42.

C. Altered biotite from the zircon mine, Green River, Henderson County, N. C. Analysis by E. A. Schneider. Disçussed by Clarke and Schneider in Bulletin 90.

D. Kerrite, from near Franklin, Macon County, N. C. Analysis by E. A. Schneider. Discussed by Clarke and Schneider in Bulletin 78.

E. Lucasite. Described as a new species by. Chatard in Bulletin 42. Analysis by T. M. Chatard. From Corundum. Hill, North Carolina. Specific gravity, 2.613 at $25.5^{\circ}$.

\begin{tabular}{|c|c|c|c|c|c|}
\hline & A. & B. & C. & D. & E. \\
\hline $\mathrm{SiO}_{2 \ldots}$ & 34.20 & 37.96 & 38.18 & 38.13 & 39.81 \\
\hline $\mathrm{TiO}_{2} \ldots \ldots$ & $\ldots$ & ........ & 1.68 & $\ldots$ & - . . . . . \\
\hline $\mathrm{Al}_{2} \mathrm{O}_{3} \ldots \ldots$ & 16.58 & 22.53 & 14.02 & 11.22 & 12.99 \\
\hline $\mathrm{Cr}_{2} \mathrm{O}_{3} \ldots \ldots \ldots$ & .... & $\cdots$ & $\cdots$ & $\ldots$. & .54 \\
\hline $\mathrm{Fe}_{2} \mathrm{O}_{3} \ldots \ldots \ldots$ & 7.41 & 11.12 & 13.02 & 2.28 & 5.29 \\
\hline $\mathrm{FeO} \ldots$ & 1.13 & .30 & 2.22 & $.18^{\circ}$ & .11 \\
\hline $\mathrm{MnO}$ & $\ldots$ & .12 & .38 & $\ldots$ & .05 \\
\hline $\mathrm{NiO}: \ldots$ & & ........ & $\ldots$ & .48 & $\ldots$ \\
\hline $\mathrm{CoO} \ldots$ & & 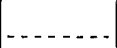 & & trace & .... \\
\hline $\mathrm{CaO} \ldots$. & & none & .17 & & .14 \\
\hline $\mathrm{BaO}$ & . & $\ldots$ & .06 & $\ldots$ & $\ldots$ \\
\hline $\mathrm{MgO} \ldots .$. & $20 . \dot{4} 1$ & 15.46 & 14.62 & .27 .39 & 24.83 \\
\hline $\mathrm{Na}_{2} \mathrm{O}_{-}$ & & undet. & .48 & & .20 \\
\hline $\mathrm{K}_{2} \mathrm{O} \ldots \ldots$ & & undet. & 5.40 & & 5.76 \\
\hline $\mathrm{H}_{2} \mathrm{O}$ at $105^{\circ}$ & 21.14 & 1268 & $3.20^{\circ}$ & $204^{17}$ & 3.78 \\
\hline $\mathrm{H}_{2} \mathrm{O}$ above $105^{\circ} \ldots$ & 21.14 & 12.63 & 7.32 & 20.47 & 6.98 \\
\hline · & 100.87 & 100.12 & 100.75 & 100.15 & 100.48 \\
\hline
\end{tabular}


F. Hallite from Nottingham, Chester County, Pa.

G. White lennilite from Lenni, Delaware County, Pa.

H. Brown lennilite, same locality.

I. Green lennilite, same locality.

Analyses F to I by E. A. Schneider. Discussion by Clarke and Schneider in Bulletin 90.

\begin{tabular}{|c|c|c|c|c|}
\hline · & F. & G. & $\mathrm{H}$. & I. \\
\hline $\mathrm{SiO}_{2} \ldots$ & 35.54 & 36.72 & 35.09 & 34.90 \\
\hline $\mathrm{TiO}_{2}$ & undet. & .18 & .58 & .10 \\
\hline $\mathrm{Al}_{2} \mathrm{O}_{3}$ & 9.74 & 10.06 & 12.05 & 10.60 \\
\hline $\mathrm{Fe}_{2} \mathrm{O}_{3}$ & 9.07 & 5.37 & 6.67 & 8.57 \\
\hline $\mathrm{Cr}_{2} \mathrm{O}_{3} \ldots \ldots$ & $\ldots$ & .26 & .46 & .23 \\
\hline $\mathrm{FeO} \ldots \ldots$ & .28 & .12 & .11 & .22 \\
\hline MnÓ ...... & .25 & .31 & .27 & .17 \\
\hline $\mathrm{NiO} \ldots \ldots . . . .$. & .16 & . . 20 & .20 & .19 \\
\hline MgO $\ldots \ldots \ldots$ & 30.05 & 29.40 & 27.62 & 28.21 \\
\hline $\mathrm{BaO} \ldots$ & $\ldots$ & $\ldots$ & trace & $\ldots$ \\
\hline $\mathrm{H}_{2} \mathrm{O}$ at $105^{\circ}$. & 2.64 & 6.40 & 5.70 & 4.99 \\
\hline \multirow[t]{2}{*}{$\mathrm{H}_{2} \mathrm{O}$ above $105^{\circ}$} & 12.14 & 11.37 & 11.20 & 11.48 \\
\hline & 99.87 & 100.39 & 99.95 & 99.66 \\
\hline
\end{tabular}

Bull. 220-03-6 
J. A vermiculite from Newlin, Chester County, Pa.

K. Painterite from Midadleton, Delaware County, Pa.

L. Another sample of painterite.

Analyses J, K, L by E. A. Schneider. Discussion by Clarke and Schneider in Bulletin 90.

M. Hydromica from Rocky Hill, N. J. Analysis by George Steiger. Described by Clarke and Darton in Bulletin 167.

$\mathrm{N}$. Analysis M corrected by deduction of calcite, union of like radicals, and recalculation to 100 per cent.

O. Protovermiculite from Magnet Cove, Arkansas. Analysis by E. A. Schneider. Discussed by Clarke and Schneider in Bulletin 90.

\begin{tabular}{|c|c|c|c|c|c|c|}
\hline & J. & $\mathrm{K}$. & L. & M. & N. & o. . \\
\hline $\mathrm{SiO}_{2-}$ & 31.23 & 34.86 & 33.95 & 32.72 & 40.24 & 34.03 \\
\hline $\mathrm{TiO}_{2} \ldots \ldots$ & . & trace & trace & .24 & ... & undet. \\
\hline $\mathrm{Al}_{2} \mathrm{O}_{3} \ldots \ldots$ & 187.52 & 11.64 & 12.52 & 8.41 & 10.34 & 14.49 \\
\hline $\mathrm{Cr}_{2} \mathrm{O}_{3} \ldots \ldots$ & .14 & & & & & - . - \\
\hline $\mathrm{Fe}_{2} \mathrm{O}_{3}$ & 4.70 & 3.78 & 4.40 & 19.99 & 24.57 & 7.71 \\
\hline $\mathrm{FeO} \ldots \ldots$ & 1.20 & .20 & .20 & 4.24 & 5.21 & .14 \\
\hline $\mathrm{MnO} \ldots \ldots$ & .20 & & & & & .09 \\
\hline $\mathrm{NiO}_{\ldots} \ldots \ldots$ & .33 & .14 & .23 & $\ldots$ & & $\ldots$ \\
\hline $\mathrm{CaO} \ldots$ & . & .07 & none & 10.30 & $\ldots$ & 1.88 \\
\hline MgO $\ldots . . . .$. & 31.36 & 31.32 & 30.56 & 5.51 & 6.78 & 20.89 \\
\hline $\mathrm{Na}_{2} \mathrm{O} \ldots$ & & & & .63 & | & ...... \\
\hline $\mathrm{K}_{2} \mathrm{O}_{\ldots} \ldots \ldots$ & $\ldots$ & $\ldots . .$. & $\ldots$ & $\cdot .85$ & 2.20 & $\ldots$ \\
\hline $\mathrm{H}_{2} \mathrm{O}$ at $105^{\circ} \ldots$ & 1.08 & 1.64 & 1.56 & 2.47 & 3.03 & 11.23 \\
\hline $\mathrm{H}_{2} \mathrm{O}$ above $105^{\circ}$ & 12.55 & 16.78 & 17.05 & 6.22 & 7.63 & 9.96 \\
\hline \multirow[t]{2}{*}{$\mathrm{CO}_{2}$} & & & (....... & 8.21 & $\ldots$ & ... \\
\hline & 100.31 & 100.43 & 100.47 & 99.79 & 100.00 & 100.42 \\
\hline
\end{tabular}


P. Chloritic vermiculite from Corundum Hill, North Carolina.

Q. Altered chlorite from Corundum Hill. Analyses P, Q by 'T. M. Chatard. Description in Bulletin 42.

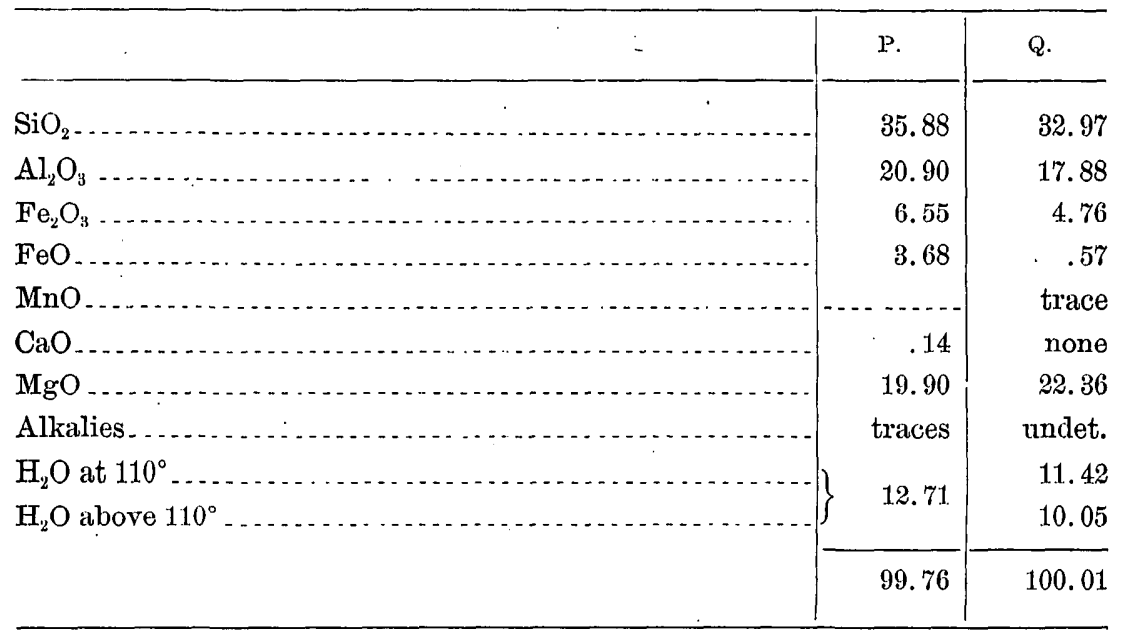

\section{CLINOCHLORE.}

A. From Westchester, Pa. Broadly foliated. Analysis by E. A. Schneider. Discussed by Clarke and Schneider in Bulletin 78.

B. From the Nikolai-Maximilian mine, district of Slatoust, Urals, Siberia. Analysis by E. A. Schneider. Discussed by Clarke and Schneider in Bulletin 113.

C. Leuchtenbergite from Slatoust. Analyst and reference as under $B$.

D. Kotschubeite from Green Valley, American River: Canyon, California. Specific gravity, 2.69. Analysis by W. H. Melville: Described by Melville and Lindgren in Bulletin 61.

\begin{tabular}{|c|c|c|c|c|}
\hline & A. & 3. & C. & D. \\
\hline $\mathrm{SiO}_{2} \ldots \ldots \ldots$ & 29.87 & 30.84 & 30.00 & 31.74 \\
\hline $\mathrm{Al}_{2} \mathrm{O}_{3} \ldots \ldots$ & 14.48 & 18.31 & 20.43 & 6.74 \\
\hline $\mathrm{Co}_{2} \mathrm{O}_{3} \ldots$ & 1.56 & 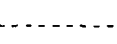 & (n...... & 11.39 \\
\hline $\mathrm{Fe}_{2} \mathrm{O}_{3}$ & 5.52 & 1.94 & 1.68 & -1 \\
\hline $\mathrm{FeO} \ldots$ & 1.93 & 1.08 & .14 & 1.23 \\
\hline $\mathrm{NiO} \ldots \ldots$ & .17 & $\cdots$ & $\ldots$ & .49 \\
\hline $\mathrm{CaO}$ & $\cdots$ & $\ldots$ & .21 & .18 \\
\hline $\mathrm{MgO} \ldots$ & 33.06 & 34.38 & 34.26 & 35.18 \\
\hline $\mathrm{H}_{2} \mathrm{O}$ at $105^{\circ}$. & 1360 & .55 & .55 & .37 \\
\hline \multirow[t]{2}{*}{$\mathrm{H}_{2} \mathrm{O}$ above $105^{\circ}$. } & 10.00 & 13.33 & 13.20 & 12.68 \\
\hline & 100.19 & 100.43 & 100.47 & 100.00 \\
\hline
\end{tabular}


PROCHLORITE.

From the Aqueduct Tunnel, Washington, D. C. Analysis by E. A. Schneider. Described by Clarke and Schneider in Bulletin 78.

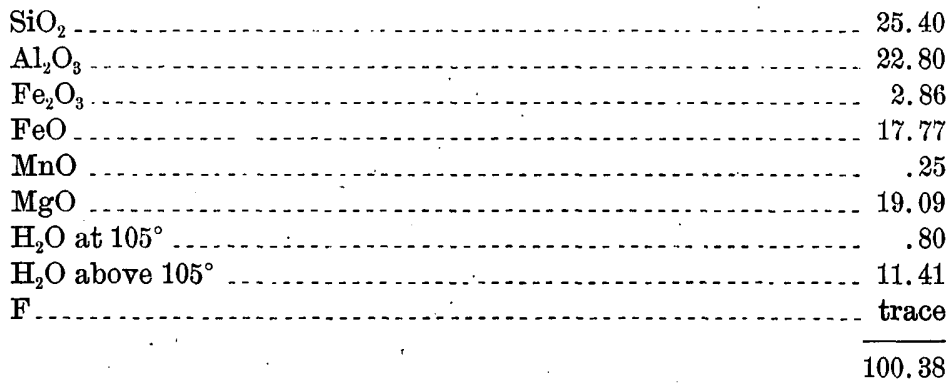

\section{SERPENTINE.}

A. From Newburyport, Mass. Analysis by F. W. Clarke.

B. Same locality as A. Analysis by E. A. Schneider. Discussion by Clarke and Schneider in Bulletin 78.

C. Serpentine derived from sahlite, Osburn's soapstone quarry, Blandford, Mass. Analysis by W. F. Hillebrand.

D. Dark-green, oily serpentine, Middlefield, Mass. Analysis by W. F. Hillebrand.

E. Serpentine derived from enstatite, Granville, Mass.

Analysis by George Steiger. Serpentines C, D, E are described by Emerson in Monograph XXIX.

\begin{tabular}{|c|c|c|c|c|c|}
\hline & A. & B. & C. & D. & E. \\
\hline $\mathrm{SiO}_{2--}$ & 41.32 & 41.47 & 40.77 & 38.62 & 37. \\
\hline $\mathrm{TiO}_{2} \ldots \ldots$ & & $\cdots$ & none & none & tre \\
\hline $\mathrm{Al}_{2} \mathrm{O}_{3} \ldots \ldots$ & & $\cdots$ & 1.16 & .35 &. \\
\hline $\mathrm{Cr}_{2} \mathrm{O}_{3}$ & & $\ldots$ & .28 & .39 & . \\
\hline $\mathrm{Fe}_{2} \mathrm{O}_{3}$ & $2 \ldots$ & 1.73 & 3.56 & 3.44 & 7. \\
\hline $\mathrm{FeO}$. & 2.36 & .09 & 1.47 & 3.99 & 1. \\
\hline $\mathrm{MnO}$ & & $\ldots$ & .09 & .10 & \\
\hline $\mathrm{NiO}$ & & - . & .17 & .21 & .4 \\
\hline $\mathrm{CoO} \ldots$ & & ...... & (n. & $\ldots$ &. \\
\hline $\mathrm{CaO}$ & . . & none & none & .40 & no \\
\hline $\mathrm{MgO}$. & 41.49 & 41.70 & 39.37 & 40.61 & 37. \\
\hline $\mathrm{Li}_{2} \mathrm{O}$ & $\ldots$ & . & trace & trace & \\
\hline $\mathrm{Na}_{2} \mathrm{O} \ldots$ & & & .14 & .10 & $\operatorname{trac}$ \\
\hline $\mathrm{K}_{2} \mathrm{O}$ & & . & .10 & .08 & trac \\
\hline $\mathrm{H}_{2} \mathrm{O}$ at $110^{\circ} \ldots$ & 14. & 1. 20 & .49 & .36 & .75 \\
\hline $\mathrm{H}_{2} \mathrm{O}$ above $110^{\circ}$ & $14_{0} 0 \pm$ & 13.86 & 12.48 & 10.91 & 12.50 \\
\hline $\mathrm{P}_{2} \mathrm{O}_{5}$ & & & trace & trace & trac \\
\hline $\mathrm{CO}_{2} \ldots \ldots$ & & & none & .52 & \\
\hline & 99.71 & 100.05 & 100.08 & 100.08 & 99. \\
\hline
\end{tabular}


F. Rich, dark-green serpentine, Rowe, Mass.

G. Black serpentine containing marmolite, Atwater's quarry, Russell, Mass.

H. Blackish-green serpentine, containing much chromite, North Blandford, Mass.

I. Gray, splintery serpentine, Chester, Mass. Analyses F to I by George Steiger. Description by Emerson in Monograph XXIX.

J. Black, serpentized boltonite, Stow, Mass. Analysis by W. F. Hillebrand.

\begin{tabular}{|c|c|c|c|c|c|}
\hline & F. & G. & H. & I. & $\mathrm{J}$ \\
\hline $\mathrm{SiO}_{2}$. & 40.42 & 36.94 & 39.14 & 33.87 & 36.92 \\
\hline $\mathrm{TiO}_{2} \ldots$ & none & trace & none & none & none \\
\hline $\mathrm{Al}_{2} \mathrm{O}_{3}$ & 1.86 & .50 & 1.18 & .77 & .10 \\
\hline $\mathrm{Cr}_{2} \mathrm{O}_{3} \ldots \ldots$ & .28 & .33 & .33 & .38 & none \\
\hline $\mathrm{Fe}_{2} \mathrm{O}_{3}-$ & 2.75 & 6.04 & 4.46 & 2.81 & 1.19 \\
\hline $\mathrm{FeO} \ldots$ & 4.27 & 1.94 & 3.14 & 4.25 & .87 \\
\hline $\mathrm{MnO} \ldots$ & trace & trace & none & .04 & trace \\
\hline $\mathrm{NiO} \ldots . . .$. & .53 & .40 & .47 & 33 & \\
\hline $\mathrm{CoO} \ldots$ & trace & none & $\operatorname{trac} \theta$ & .00 & ....... \\
\hline $\mathrm{CaO} \ldots$. & .66 & none & none & none & .59 \\
\hline $\mathrm{MgO} \ldots \ldots$ & 35.95 & 38.35 & 41.45 & 38.57 & 43.99 \\
\hline $\mathrm{Na}_{2} \mathrm{O} \ldots \ldots$ & 16 & none & none & none & .05 \\
\hline $\mathrm{K}_{2} \mathrm{O} \ldots$ & & none & none & none & \} \\
\hline $\mathrm{H}_{2} \mathrm{O}$ at $100^{\circ} \ldots \ldots$ & .21 & .71 & .34 & .38 & .72 \\
\hline $\mathrm{H}_{2} \mathrm{O}$ above $100^{\circ} \ldots$ & 10.51 & 12.07 & 9.48 & 7.00 & 14.70 \\
\hline $\mathrm{P}_{2} \mathrm{O}_{5} \ldots$ & trace & trace & .02 & trace & trace \\
\hline $\mathrm{CO}_{2}$ & 1.44 & 1.85 . & none. & 10.82 & .90 \\
\hline $\mathrm{SO}_{3} \ldots \ldots$ & trace & .20 & none & .20 & \\
\hline \multirow[t]{2}{*}{$\mathrm{FeS}_{2}$} & .43 & & & & ....... \\
\hline & 99.47 & 99.33 & 100.01 & 99.42 & 100.03 \\
\hline
\end{tabular}


.K. From Moriah, N. Y. Analysis by Charles Catlett. Described by Merrill, Proc. U. S. Nat. Mus., vol. 12, p. 596.

L. From the aqueduct shaft, New York City. Analysis by Charles Catlett. See Merrill, loc. cit., p. 598.

M. From Montville, N. J., light yellowish-green.

N. From Montville, darker green. Analyses M, N, by Charles Catlett. See Merrill, Proc., U. S. Nat. Mus., vol. 11, p. 105.

O. From Montville, massive.

P. From Montville, chrysotile. Analyses O, P, by E. A. Schneider. Discussion by Clarke and Schneider in Bulletin 78 .

\begin{tabular}{|c|c|c|c|c|c|c|}
\hline & K. & L. & M. & N. & 0. & P. \\
\hline $\mathrm{SiO}_{2} \ldots \ldots$ & 39.96 & 39.92 & 42.38 & 40.23 & 42.05 & 42.42 \\
\hline $\mathrm{Al}_{2} \mathrm{O}_{3}$ & 1.07 & .08 & .07 & 2.18 & 30 & .63 \\
\hline $\mathrm{Fe}_{2} \mathrm{O}_{3}-$ & 3.53 & .50 & .97 & 4.02 & .00 & .62 \\
\hline $\mathrm{FeO}$ & 3.85 & none & .17 & trace? & .10 & undet. \\
\hline $\mathrm{MnO}$ & trace & & & & & none \\
\hline $\mathrm{NiO}$ & none & & & & & .23 \\
\hline $\mathrm{CaO}$ & none & .90 & $\ldots$ & trace & .05 & trace \\
\hline $\mathrm{MgO}_{-}$ & 37.61 & 42.52 & 42.14 & 39.46 & 42.57 & 41.01 \\
\hline \multirow{4}{*}{$\begin{array}{l}\mathrm{H}_{2} \mathrm{O} \text { at } 105^{\circ} \\
\mathrm{H}_{2} \mathrm{O} \text { above } 105^{\circ} \\
\mathrm{CO}_{2}\end{array}$} & 126 & 1.36 & 1412 & 14.94 & .96 & 2.04 \\
\hline & 13.00 & 13.26 & 14.12 & 14.24 & 13.70 & 13.60 \\
\hline & & 1.64 & & & $\ldots$ & $\ldots$ \\
\hline & 99.67 & 100.18 & 99.85 & 100.13 & 99.73 & 100.55 \\
\hline
\end{tabular}


Q. From Easton, Pa. Resembles a vermiculite. Analysis by E. A. Schneider. See Clarke and Schneider, Bulletin 90.

R. Grayish-green serpentine from Corundum Hill, North Carolina.

S. Picrolite from Buck Creek, Clay County, N. C. Analyses R and $\mathrm{S}$ by E. A. Schneider. See Clarke and Schneider, Bulletin 78.

T. From the river Poldnewaja, district of Syssert, Urals, Siberia. Analysis by E. A. Schneider. See Clarke and Schneider, Bulletin 113.

U. From Greenville, Plumas County, Cal. Analysis by W. H. Melville. Described by Diller in Bulletin 150.

\begin{tabular}{|c|c|c|c|c|c|}
\hline & Q. & R. & s. & T. & U. \\
\hline $\mathrm{SiO}_{2} \ldots$ & 43.71 & 41.90 & 42.94 & 42.55 & 39.14 \\
\hline $\mathrm{Al}_{2} \mathrm{O}_{3} \ldots \ldots \ldots$ & 3.59 & .71 & \multirow{2}{*}{5.05} & 1.25 & 2.08 \\
\hline $\mathrm{Fe}_{2} \mathrm{O}_{3}$ & .90 & \multirow{4}{*}{$\begin{array}{r}.91 \\
\text { undet. } \\
.10\end{array}$} & & 1.56 & 4.27 \\
\hline $\mathrm{FeO} \ldots \ldots$ & $\cdots$ & & \multirow{3}{*}{$\begin{array}{r}1.88 \\
.61\end{array}$} & 1.52 & \multirow{2}{*}{$\begin{array}{r}2.04 \\
-\ldots .\end{array}$} \\
\hline $\mathrm{NiO} \ldots \ldots . . .$. & $\ldots$ & & & & \\
\hline $\mathrm{CaO} \ldots$ & $\cdots$ & & & none & trace \\
\hline $\mathrm{MgO} \ldots \ldots$ & 38.58 & \multirow[t]{2}{*}{40.16} & \multirow[t]{2}{*}{36.53} & 40.05 & 39.84 \\
\hline $\mathrm{Na}_{2} \mathrm{O}_{2} \ldots \ldots$ & .13 & & & & \\
\hline $\mathrm{K}_{2} \mathrm{O} \ldots \ldots$ & 2.22 & $\cdots$ & & & \multirow{3}{*}{12.70} \\
\hline $\mathrm{H}_{2} \mathrm{O}$ at $105^{\circ} \ldots$ & .46 & 2.26 & 1.53 & .21 & \\
\hline $\mathrm{H}_{2} \mathrm{O}$ above $105^{\circ}$ & 10.79 & 13.90 & 11.68 & 12.26 & \\
\hline \multirow[t]{2}{*}{ Chromite. } & & & & .37 & .11 \\
\hline & $100: 38$ & 99.94 & 100.22 & 99.77 & 100.18 \\
\hline
\end{tabular}


V. Light-green marmolitic serpentine, New Idria, Cal.

W. Black serpentine, Sulphur Bank, California.

X. Light-green serpentine, Sulphur Bank.

Analyses V, W, X by W. H. Melville. Described by Becker in Monograph XIII. Other analyses by Melville of impure serpentines from near Mount Diablo, California, are given in Bulletin 168.

Y. From Three Brothers, Mount Stuart quadrangle, Washington. Analysis by W. F. Hillebrand.

\begin{tabular}{|c|c|c|c|c|}
\hline & $\mathrm{V}$. & w. & $\mathrm{x}$. & $\mathbf{Y}$. \\
\hline $\mathrm{SiO}_{2} \ldots \ldots$ & \multirow[t]{2}{*}{41.54} & \multirow{2}{*}{$\begin{array}{l}39.64 \\
\ldots . . .\end{array}$} & \multirow{2}{*}{$\begin{array}{c}41.86 \\
\ldots \ldots\end{array}$} & 39.00 \\
\hline $\mathrm{TiO}_{2} \ldots \ldots$ & & & & trace \\
\hline $\mathrm{Al}_{2} \mathrm{O}_{3}$ & \multirow[t]{2}{*}{2.48} & \multirow{3}{*}{$\begin{array}{r}1.30 \\
.29 \\
\end{array}$} & \multirow{3}{*}{$\begin{array}{l}.69 \\
.24 \\
.\end{array}$} & 1.75 \\
\hline $\mathrm{Cr}_{2} \mathrm{O}_{3}$ & & & & .47 \\
\hline $\mathrm{Fe}_{2} \mathrm{O}_{3} \ldots \ldots$ & -- & & & 5.16 \\
\hline $\mathrm{FeO} \ldots \ldots \ldots$ & \multirow{2}{*}{1.37} & \multirow{4}{*}{$\begin{array}{r}7.76 \\
.12 \\
.33 \\
\ldots . . .\end{array}$} & \multirow{3}{*}{$\begin{array}{r}4.15 \\
.20 \\
\text { trace }\end{array}$} & 1.71 \\
\hline $\mathrm{MnO} \ldots \ldots$ & & & & .15 \\
\hline 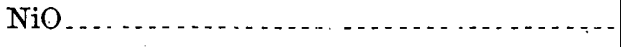 & \multirow[t]{2}{*}{.04} & & & .10 \\
\hline $\mathrm{CaO} \ldots \ldots$ & & & -....... & trace \\
\hline $\mathrm{MgO}$ & \multirow[t]{2}{*}{40.42} & \multirow{2}{*}{37.13} & \multirow[t]{2}{*}{38.63} & 38.00 \\
\hline Alkalies.... & & & & .10 \\
\hline $\mathrm{H}_{2} \mathrm{O}$ at $110^{\circ} \ldots$ & \multirow{3}{*}{14.18} & \multirow{3}{*}{13.81} & \multirow{3}{*}{14.16} & 1.31 \\
\hline $\mathrm{H}_{2} \mathrm{O}$ above $110^{\circ}-\ldots$ & & & & 12.43 \\
\hline $\mathrm{P}_{2} \mathrm{O}_{5} \ldots \ldots$ & & & & trace \\
\hline \multirow[t]{2}{*}{$\mathrm{FeS}_{2}$} & & 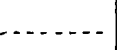 & & .03 \\
\hline & 100.03 & 100.38 & 99.93 & 100.21 \\
\hline
\end{tabular}

GENTHITE.

From Riddle, Douglas County, Oreg. Analyzed by F. W. Clarke, and described by Diller and Clarke in Bulletin 60 .

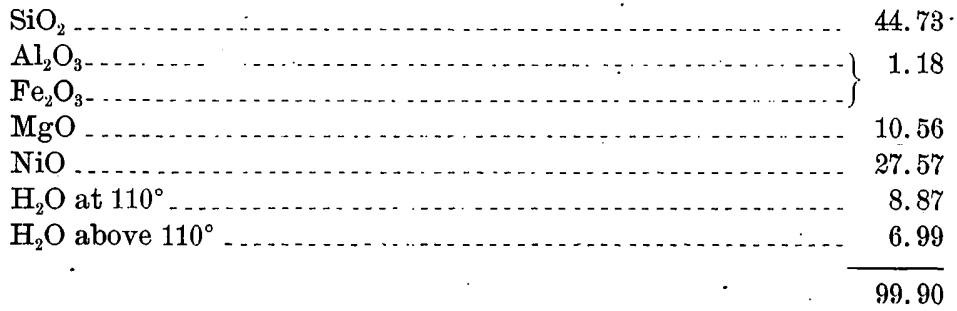


TALC.

Apple-green, beautifully foliated. From Huntersville, Fairfax County, Va. Analysis by E. A. Schneider.

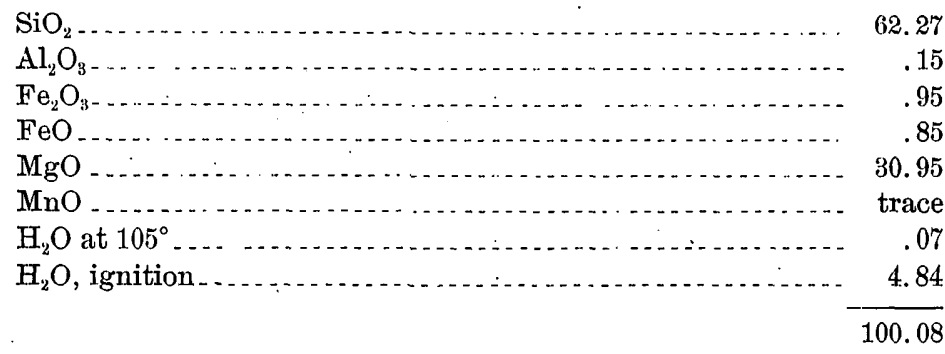

\section{GIJAUCONITE.}

From Big Goose Canyon, 15 miles southwest of Sheridan, Big Horn Mountains, Wyoming. Analysis by George Steiger. Specific gravity, 2.73 .

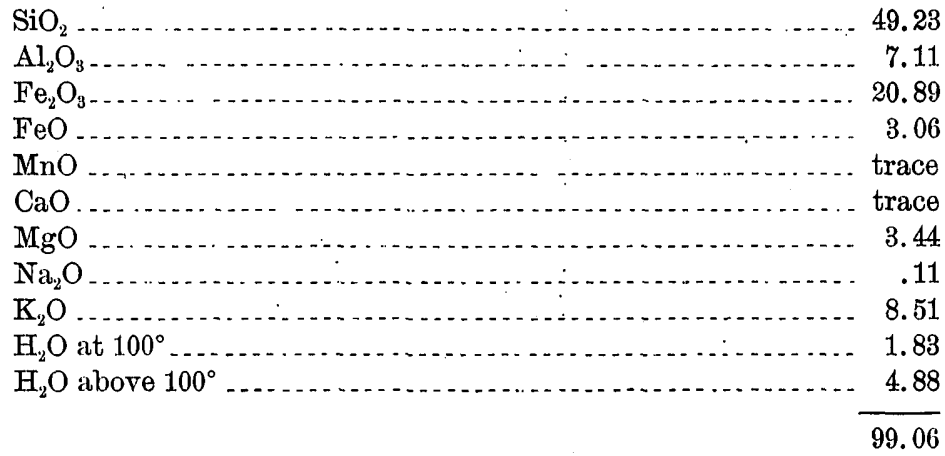


KAOLINITE.

A. From Hockessen; Del. Analysis by George Steiger.

B. From Aiken, S. C. Analysis by F. W. Clarke.

C. From Talladega, Ala. Analysis by Charles Catlett.

D. From the Eureka vein, St. Peter's Dome, near Pikes Peak, Colorado. Analysis by W. F. Hillebrand. Described by Cross and Hillebrand in Bulletin 20.

E. From New Discovery mine, Leadville, Colo. Analysis by W. F. Hillebrand.

F. From National Belle mine, Ouray County, Colo. Analysis by W. F. Hillebrand. Specific gravity, 2.611 at $18.5^{\circ}$. Described in Bulletin 20 .

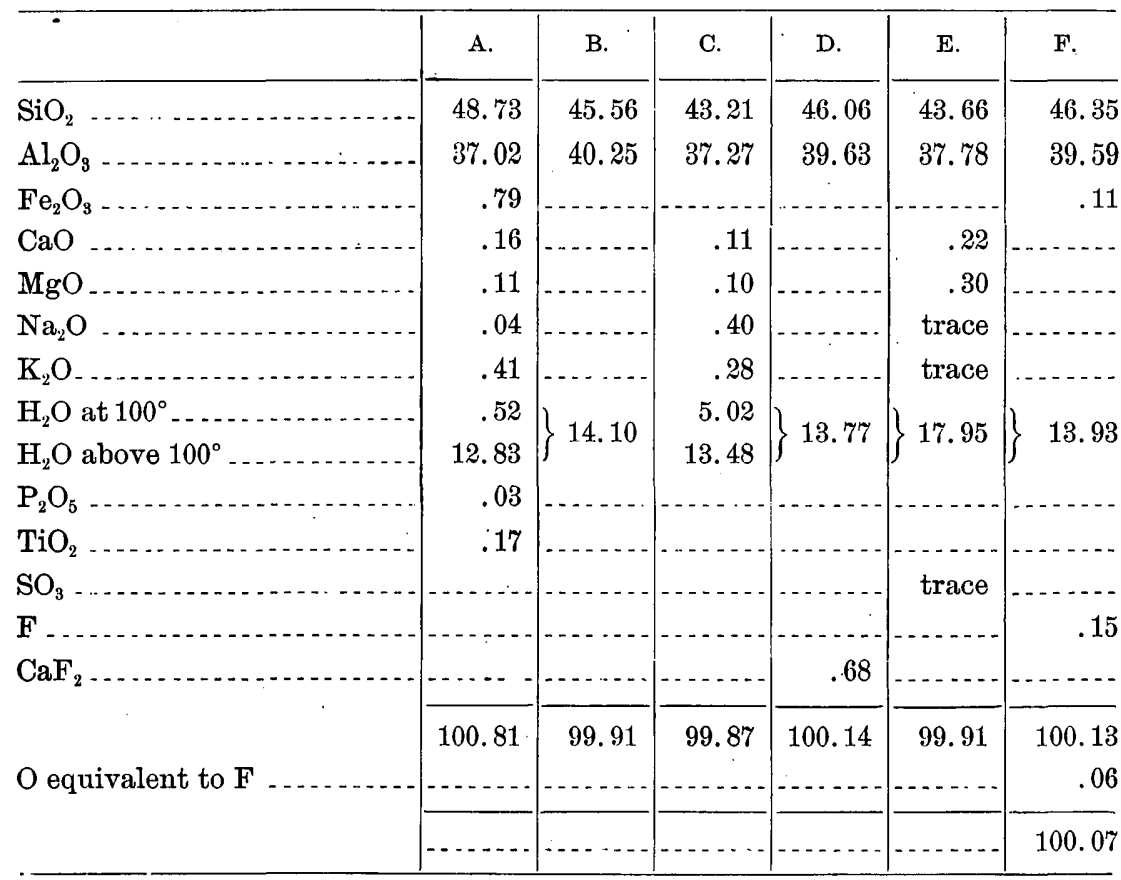

HALLOYSITE.

A. From the Detroit copper mine, near Mono Lake, California. Analyzed by F. W. Clarke, and described in Bulletin 9 .

B. From the Logan mine, Rico, Colo. Analysis by W. F. Hillebrand.

\begin{tabular}{|c|c|c|}
\hline & A. & B. \\
\hline $\mathrm{SiO}_{2}-\ldots$ & 42.91 & 38.65 \\
\hline $\mathrm{Al}_{2} \mathrm{O}_{3}$ & 38.13 & 33.27 \\
\hline $\mathrm{Fe}_{2} \mathrm{O}_{3} \ldots \ldots \ldots$ & & .22 \\
\hline $\mathrm{H}_{2} \mathrm{O}$ at $100^{\circ} \ldots$ & 18.95 & 13.70 \\
\hline \multirow[t]{2}{*}{$\mathrm{H}_{2} \mathrm{O}$ above $100^{\circ}$. } & 10.95 & 14.34 \\
\hline & 99.99 & 100.18 \\
\hline
\end{tabular}




\section{CIMOLITE.}

Rose-red, earthy variety, from Norway, Me. Aualysis by R. B. Riggs. Described by Clarke in Bulletin 42.

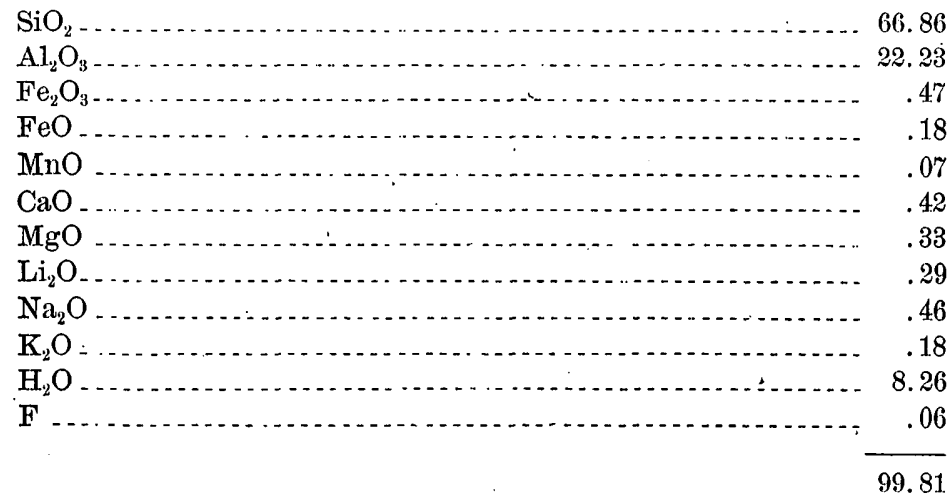

PYROPHYLLITE.

Compact, white. From Deep River, North Carolina. Analysis by George Steiger.

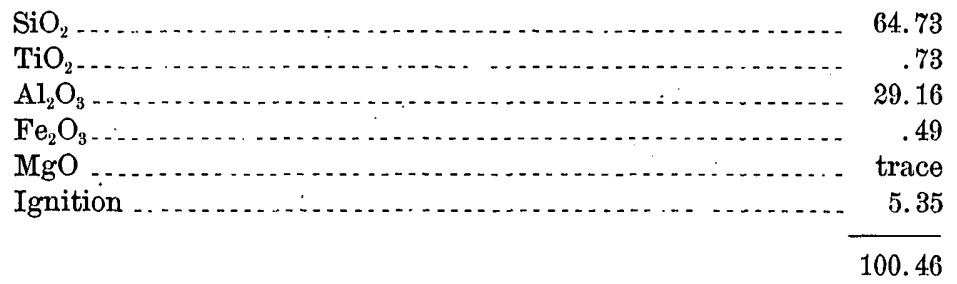

BOLE.

From South Table Mountain, Golden, Colo. Described by Cross and Hillebrand in Bulletin 20. Analyses by W. F. Hillebrand.

A. Dark brown.

B. Light brown.

\begin{tabular}{|c|c|c|}
\hline & A. & B. \\
\hline $\mathrm{SiO}_{2}$ & 42.63 & 46.17 \\
\hline $\mathrm{Al}_{2} \mathrm{O}_{3}$ & 18.76 & 22.03 \\
\hline $\mathrm{Fe}_{2} \mathrm{O}_{3}^{-}$ & 11.88 & 4.64 \\
\hline $\mathrm{CaO} \ldots \ldots \ldots$ & 2.59 & 2.30 \\
\hline $\mathrm{MgO}$ & 3.39 & 2.42 \\
\hline $\mathrm{K}_{2} \mathrm{O}$ & .35 & a2.06 \\
\hline $\mathrm{Na}_{2} \mathrm{O}$ & .24 & \\
\hline \multirow{2}{*}{$\mathrm{H}_{2} \mathrm{O}$} & 20.21 & 20.38 \\
\hline & 100.05 & 100.00 \\
\hline
\end{tabular}




\section{TITANATES AND TITANO-SIJICATES.}

ILMENITE.

From the peridotite dike of Elliott County, Ky. Described by Diller in Bulletin 38: Analysis by T. M. Chatard.

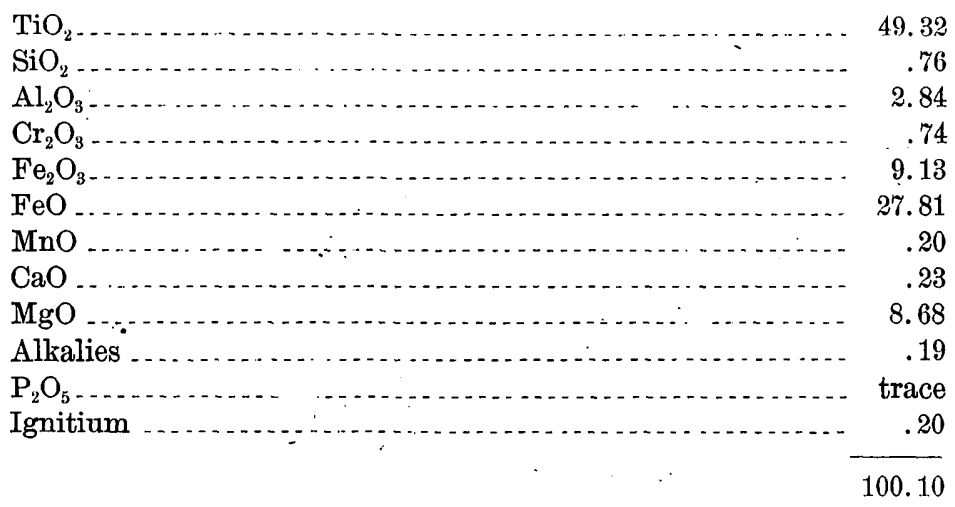

XANTHITANE.

From Green River, Henderson County, N. C. Alteration product of titanite. Analyzed by L. G. Eakins, and described in Bulletin 60 . Specific gravity, 2.941 at $24^{\circ}$. Material dried at $100^{\circ}$.

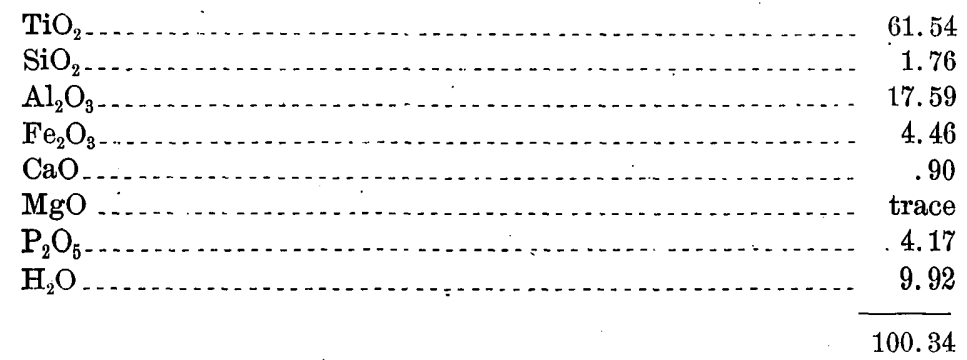

TITANITE.

Pale-yellow, semi-translucent. From the waterworks tunnel, Washington, D. C. Occurs embedded in prochlorite. Analysis by F. W. Clarke. Specific gravity, 3.452.

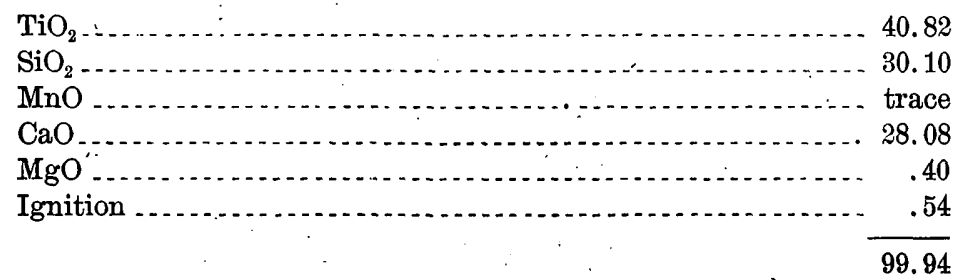




\section{TSCHEFFKINITE.}

From Bedford County, Va. Analyzed by L. G. Eakins and described by him in Bulletin 90 .

A. Lustrous portion. Specific gravity, 4.33 at $27^{\circ}$.

B. Dull portion. Specific gravity, 4.38 at $22^{\circ} .2$.

\begin{tabular}{|c|c|c|}
\hline . & A. & B. \\
\hline $\mathrm{TiO}_{2}$ & 18.78 & 18.99 \\
\hline $\mathrm{SiO}_{2} \ldots \ldots$. & 20.21 & 21.49 \\
\hline $\mathrm{Ta}_{2} \mathrm{O}_{5} \ldots$ & .08 & .08 \\
\hline $\mathrm{ZrO}_{2} \ldots \ldots$ & trace? & trace? \\
\hline $\mathrm{ThO}_{2} \ldots \ldots \ldots$ & .85 & .75 \\
\hline$(\mathrm{Y}, \mathrm{Er})_{2} \mathrm{O}_{3} \ldots$ & 1.82 & 1.64 \\
\hline$(\mathrm{La}, \mathrm{Di})_{2} \mathrm{O}_{3} \ldots \ldots$ & 19.72 & 17.16 \\
\hline $\mathrm{Ce}_{2} \mathrm{O}_{3} \ldots \ldots$ & 20.05 & 19.08 \\
\hline $\mathrm{Al}_{2} \mathrm{O}_{3}$ & 3.60 & 3.65 \\
\hline $\mathrm{Fe}_{2} \mathrm{O}_{3} \ldots \ldots$ & 1.88 & 2.89 \\
\hline $\mathrm{FeO}$ & 6.91 & 5.92 \\
\hline $\mathrm{CaO}$ & 4.05 & 5.24 \\
\hline $\mathrm{MgO} \ldots \ldots$ & .55 & .48 \\
\hline $\mathrm{Na}_{2} \mathrm{O} \ldots \ldots$ & .06 & .04 \\
\hline \multirow{2}{*}{$\mathrm{H}_{2} \mathrm{O} \ldots \ldots$} & .94 & 2.06 \\
\hline & 99.50 & 99.47 \\
\hline
\end{tabular}

ASTROPHYLILITE.

From St. Peter's Dome, near Pikes Peak, Colorado. Analyzed by L. G. Eakins and described by him in. Bulletin 90 .

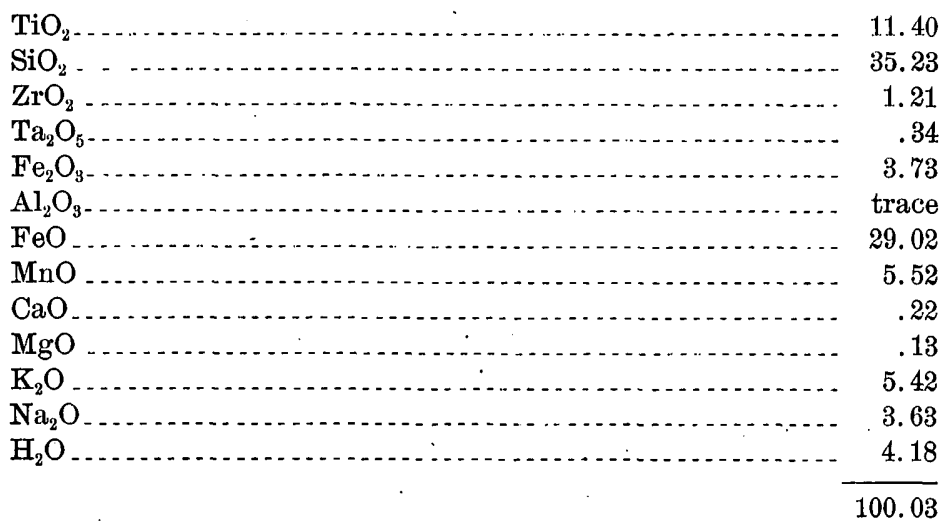




\section{COLUMBO-TAN'TALATES.}

SAMARSKITE.

Mineral near samarskite, from Devils Head Mountain, near Pikes Peak, Colorado. Analyzed by W. F. Hillebrand and described in Bulletin 55.

A. Pitch-black variety, streak dirty brown. Specific gravity, 6.18 at $22^{\circ}$.

B. Black, streak salmon colored. Specific gravity, 6.12 at $25^{\circ}$.

C. Altered variety: Specific gravity, 5.45 at $16^{\circ}$.

\begin{tabular}{|c|c|c|c|}
\hline & A. & B. & c. \\
\hline $\mathrm{Ta}_{2} \mathrm{O}_{5}$ & 27.03 & 28.11 & 19.34 \\
\hline $\mathrm{Cb}_{2} \mathrm{O}_{5} \ldots \ldots \ldots$ & 27.77 & 26.16 & 27.56 \\
\hline $\mathrm{WO}_{3} \ldots \ldots$ & 2.25 & 2.08 & 5.51 \\
\hline $\mathrm{SnO}_{2}$ & .95 & 1.09 & .82 \\
\hline $\mathrm{ZrO}_{2} \ldots \ldots \ldots \ldots$ & 2.29 & 2.60 & $a 3.10$ \\
\hline $\mathrm{UO}_{2} \ldots \ldots$ & 4.02 & 4.22 & \\
\hline $\mathrm{UO}_{3} \ldots \ldots$ & $\ldots$. & $\ldots \ldots$ & 6.20 \\
\hline $\mathrm{ThO}_{2 \ldots} \ldots \ldots$ & 3.64 & 3.60 & 3.19 \\
\hline $\mathrm{Ce}_{2} \mathrm{O}_{3} \ldots \ldots$ & .54 & .49 & .41 \\
\hline$(\mathrm{La}, \mathrm{Di})_{2} \mathrm{O}_{3} \ldots \ldots \ldots$ & 1.80 & 2.12 & 1.44 \\
\hline $\mathrm{Er}_{2} \mathrm{O}_{3}$ & 10.71 & 10.70 & 9.82 \\
\hline $\mathrm{Y}_{2} \mathrm{O}_{3} \ldots \ldots$ & 6.41 & 5.96 & 5.64 \\
\hline $\mathrm{Fe}_{2} \mathrm{O}_{3}$ & 8.77 & 8.72 & 8.90 \\
\hline $\mathrm{FeO} \ldots \ldots \ldots$ & .32 & .35 & ${ }^{b} .39$ \\
\hline $\mathrm{MnO} \ldots \ldots$ & .78 & .75 & \\
\hline $\mathrm{ZnO} \ldots \ldots$ & .05 & .07 & \\
\hline $\mathrm{PbO} \ldots \ldots$ & .72 & .80 & 1.07 \\
\hline $\mathrm{CaO} \ldots . . .$. & .27 & .33 & 1.61 \\
\hline $\mathrm{MgO}$ & $\cdots$ & & .11 \\
\hline $\mathrm{K}_{2} \mathrm{O}_{\ldots} \ldots \ldots$ & .17 & .13 & \\
\hline$(\mathrm{Na}, \mathrm{Li})_{2} \mathrm{O} \ldots \ldots$ & .24 & .17 & \\
\hline $\mathrm{H}_{2} \mathrm{O}$ & 1.58 & 1.30 & 3.94 \\
\hline \multirow[t]{2}{*}{$\mathrm{F}$} & (?) & $(?)$ & $(?)$ \\
\hline & 100.31 & 99.75 & 100.18 \\
\hline
\end{tabular}




\section{BORATES.}

\section{COLEMANITE.}

A. Transparent crystal, ordinary type, Death Valley, California.

B, C. Blade-like crystals, Death Valley.

D. Priceite, Curry County, Oreg.

D. Pandermite, Island of Panderma, Black Sea.

Analyses by J. E. Whitfield, with details in Bulletin 55.

\begin{tabular}{|c|c|c|c|c|c|}
\hline & A. & B. & C. & $\mathrm{D}$. & E. \\
\hline $\mathrm{B}_{2} \mathrm{O}_{3} \ldots$ & 50.70 & 49.56 & 49.62 & 48.44 & 48.63 \\
\hline $\mathrm{CaO} \ldots \ldots$ & 27.31 & 27.36 & 27.40 & 32.15 & 32.16 \\
\hline $\mathrm{MgO}_{-}$. & .10 & .25 & .26 & (........ & $\ldots$ \\
\hline $\mathrm{H}_{2} \mathrm{O}$. & 21.87 & 22.66 & 22.70 & 19.42 & 19.40 \\
\hline \multirow[t]{2}{*}{$\mathrm{SiO}_{2}$} & - & .44 & .47 & . & ...... \\
\hline & 99.98 & 100.27 & 100.45 & 100.01 & 100.19 \\
\hline
\end{tabular}

\section{ULEXITE.}

From Rhodes' Marsh, Esmeralda County, Nev. Analysis by J. E. Whitfield, with description in Bulletin 55.

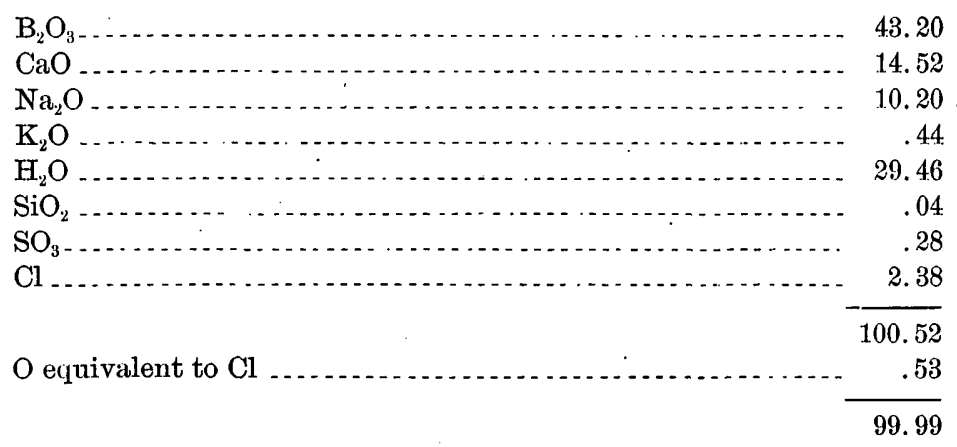

LUDWIGITE.

From Morawitza, Banat, Hungary. Analysis by J. E. Whitfield, with description in Bulletin 55.

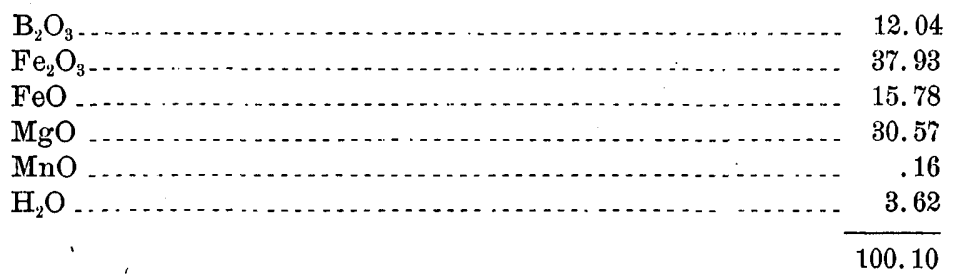


WARWICKITE.

From Edenville, Orange County, N. Y. Somewhat contaminated by spinel, from which the warwickite could not be entirely freed. Analysis by J. E. Whitfield. See Bulletin 64 .

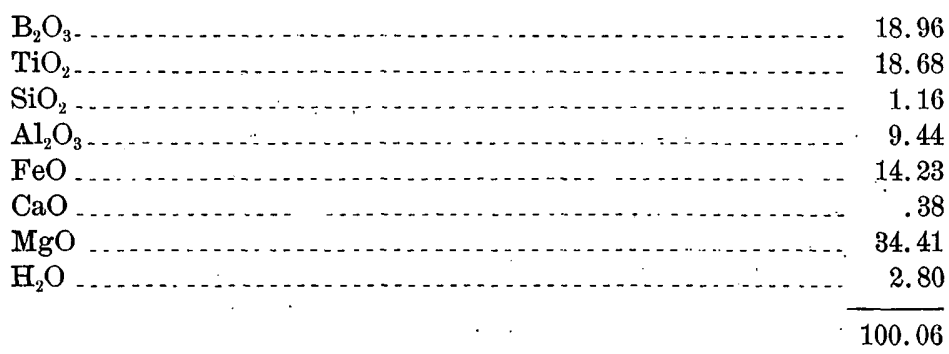

18.96

8. 68

9.44

4. 23

.38

41

2.80

XI. NITRATES.

SODA NITER.

From the Leucite Hills, Wyoming. Analysis by L. G. Eakins. The $\mathrm{N}_{2} \mathrm{O}_{5}$ was not determined directly, but calculated to satisfy $\mathrm{Na}_{2} \mathrm{O}+\mathrm{K}_{2} \mathrm{O}$.

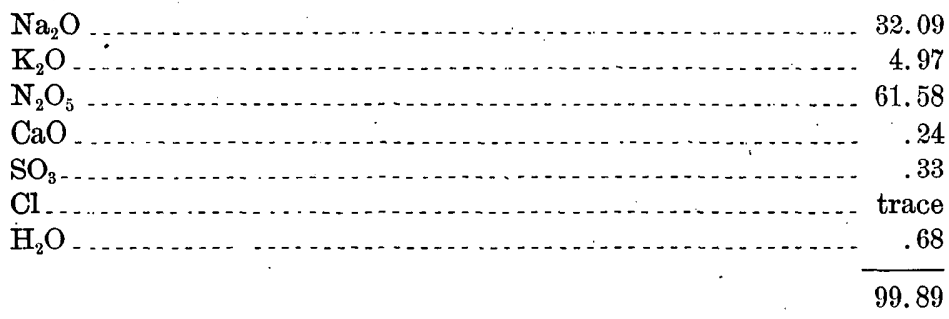

NITER.

A. From Utah, exact locality unknown. Analysis by T. M. Chatard.

B. From the Leucite Hills, Wyoming. Analysis by L. G. Eakirs, with the $\mathrm{N}_{2} \mathrm{O}_{5}$ calculated to satisfy the alkalies.

\begin{tabular}{|c|c|c|}
\hline & A. & B. \\
\hline Insoluble matter .. & 12.12 & \\
\hline $\mathrm{K}_{2} \mathrm{O} \ldots \ldots$ & 38.38 & 44.91 \\
\hline $\mathrm{Na}_{2} \mathrm{O} \ldots \ldots$ & .12 & .07 \\
\hline $\mathrm{N}_{2} \mathrm{O}_{5} \ldots \ldots$ & 44.30 & 51.49 \\
\hline $\mathrm{Si}_{2} \mathrm{O}_{3}$ & .20 & $\therefore$ \\
\hline $\mathrm{CaO}$ & 1.43 & 1.09 \\
\hline $\mathrm{MgO}$ & trace & \\
\hline $\mathrm{NaCl} \ldots \ldots$ & .08 & \\
\hline $\mathrm{Cl} \ldots$ & $\cdots$ & .09 \\
\hline $\mathrm{SO}_{3}$ & 2.05 & 1.59 \\
\hline $\mathrm{H}_{2} \mathrm{O} \ldots \ldots$ & 1.24 & .62 \\
\hline & 99.92 & 99.86 \\
\hline
\end{tabular}




\section{PHOSPHATES.}

XENOTIME.

From the gold washings at Brindletown, N. C. Analyses by T. G. Eakins.

A. Green, specific gravity, 4.68 at $24.2^{\circ}$.

B. Brown, specific gravity, 4.46 at $24.4^{\circ}$.

\begin{tabular}{|c|c|c|}
\hline & A. & B. \\
\hline $\mathrm{SiO}_{2} \ldots \ldots \ldots$ & 3.46 & 3.56 \\
\hline $\mathrm{ZrO}_{2} \ldots \ldots \ldots$ & 1.95 & 2.19 \\
\hline $\mathrm{UO}_{2} \ldots \ldots \ldots$ & 4. 13 & 1.73 \\
\hline $\mathrm{ThO}_{2} \ldots \ldots \ldots$ & trace & trace \\
\hline $\mathrm{Al}_{2} \mathrm{O}_{3}$ & .77 & 1.57 \\
\hline $\mathrm{Fe}_{2} \mathrm{O}_{3} \ldots \ldots \ldots \ldots$ & .65 & 2.79 \\
\hline$(\mathrm{La}, \mathrm{Di})_{2} \mathrm{O}_{3} \ldots \ldots$ & .93 & .77 \\
\hline$(\mathrm{Y}, \mathrm{Er})_{2} \mathrm{O}_{3} a_{-\ldots} \ldots \ldots$ & 56.81 & 55.43 \\
\hline $\mathrm{CaO}_{\ldots} \ldots \ldots . . . . . . .$. & .21 & .19 \\
\hline $\mathrm{P}_{2} \mathrm{O}_{5}$ & 30.31 & 29.78 \\
\hline$F$ & .06 & .56 \\
\hline \multirow[t]{2}{*}{$\mathrm{H}_{2} \mathrm{O} \ldots \ldots$} & .57 & 1.49 \\
\hline & 99.85 & 100.06 \\
\hline
\end{tabular}

: Molecular weight, 260 .

APATITE.

Dark-green, massive apatite from the topaz locality at Stoneham, Me. Analysis by J. E. Whitfield. See Bulletin 27. Specific gravity, 3.27.

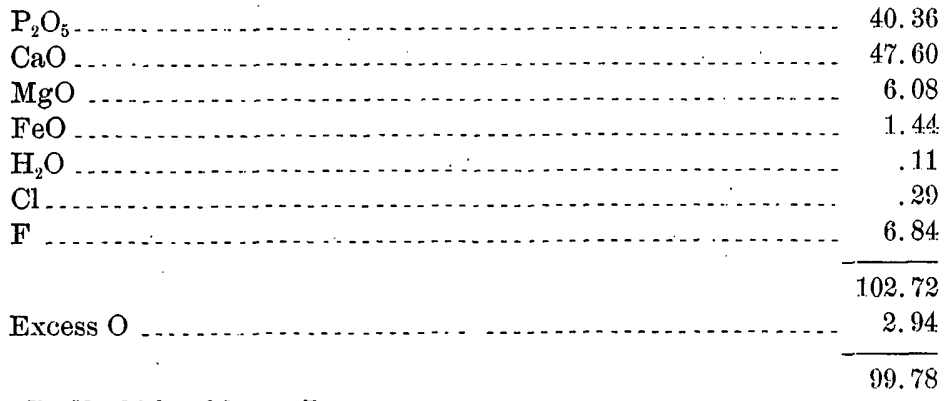

Bull. $220-03-7$

40.36

7.60

1. 4.4

11

84

102.72

99.78 


\section{TRIPLITE.}

From a tin mine near Rapid City, S. Dak. Probably identical with Headden's "griphite." Analysis by L. G. Eakins, with description in Bulletin 60 .

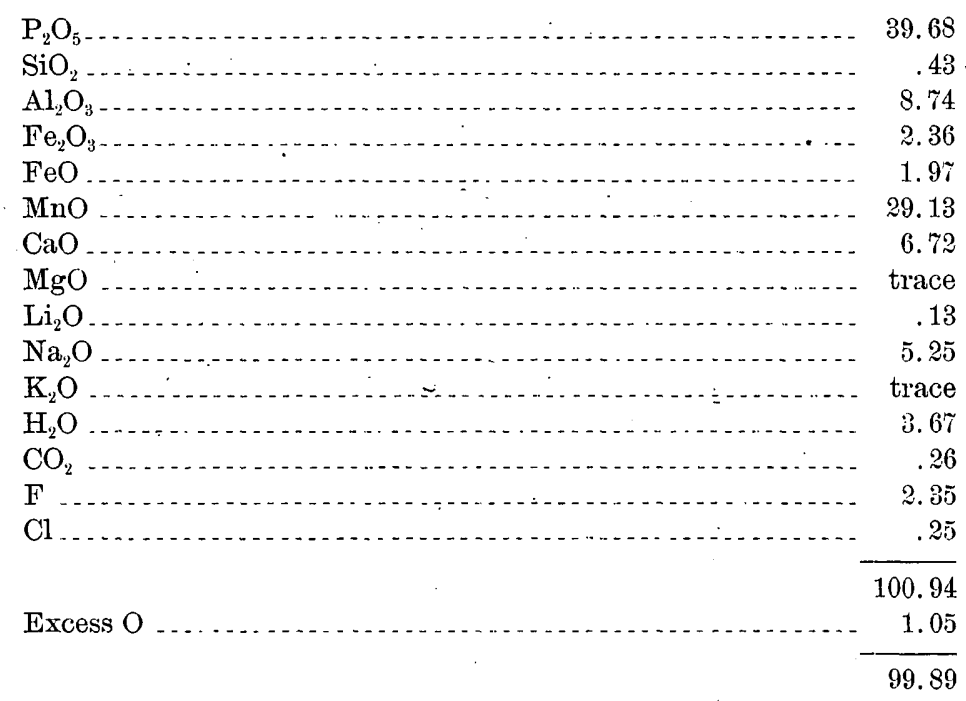

TURQUOISE.

From Los Cerillos, N. Mex. Described by Clarke and Diller in Bulletin 42. Analyses by F. W. Clarke.

A. Bright blue, faintly translucent in thin splinters.

B. Pale blue, with a slight greenish cast. Opaque and earthy in texture. Specific gravity, 2.805.

C. Dark green, opaque.

\begin{tabular}{|c|c|c|c|}
\hline . & A. & B. & C. \\
\hline $\mathrm{P}_{2} \mathrm{O}_{5}$ & 31.96 & 32.86 & 28.63 \\
\hline $\mathrm{SiO}_{2}$ & 1.15 & .16 & 4. 20 \\
\hline $\mathrm{Al}_{2} \mathrm{O}_{3} \ldots$ & 39.53 & 36.88 & 37.88 \\
\hline $\mathrm{Fe}_{2} \mathrm{O}_{3} \ldots \ldots \ldots$ & & 2.40 & 4.07 \\
\hline $\mathrm{CuO} \ldots . .$. & 6.30 & 7.51 & 6.56 \\
\hline $\mathrm{CaO} \ldots \ldots . . . . . .$. & .13 & .38 & undet. \\
\hline \multirow{2}{*}{$\mathrm{H}_{2} \mathrm{O} \ldots \ldots$} & 19.80 & 19.60 & 18.49 \\
\hline & 98.87 & 99.79 & 99.83 \\
\hline
\end{tabular}




\section{VANADATES.}

DESCLOIZITE.

A. From the Mayflower mine, Bald Mountain mining district, Beaverhead County, Mont. Yellow, friable, not crystallized.

B. From the Commercial mine, Georgetown, N. Mex. Brilliantly crystallized.

C. Cuprodescloizite from the Tucky Cuss mine, Tombstone, Ariz. Specific gravity, 5.88 at $19^{\circ}$.

Analyses by W. F. Hillebrand, with description in Bulletin 64 .

\begin{tabular}{|c|c|c|c|}
\hline$\cdot$ & A. & B. & C. \\
\hline $\mathrm{V}_{2} \mathrm{O}_{5} \ldots \ldots \ldots$ & 20.80 & 20.44 & 19.79 \\
\hline $\mathrm{As}_{2} \mathrm{O}_{5}, \ldots \ldots$ & .32 & .94 & 1.10 \\
\hline $\mathrm{P}_{2} \mathrm{O}_{5} \ldots \ldots$ & .27 & .26 & .19 \\
\hline $\mathrm{SiO}_{2} \ldots \ldots \ldots$ & .18 & 1.01 & .80 \\
\hline $\mathrm{CO}_{2} \ldots \ldots \ldots$ & $-\ldots$. & ...... & .82 \\
\hline $\mathrm{PbO} \ldots \ldots . . .$. & 55.93 & 56.01 & 57.00 \\
\hline $\mathrm{ZnO} \ldots .$. & 15.94 & 17.73 & 4.19 \\
\hline $\mathrm{CuO} \ldots \ldots \ldots$ & 1.15 & 1.05 & 11.21 \\
\hline $\mathrm{FeO} \ldots \ldots \ldots \ldots$ & .70 & .07 & trace \\
\hline $\mathrm{CaO}$ & .10 & .04 & 1.01 \\
\hline $\mathrm{MgO} \ldots$ & .06 & .03 & .04 \\
\hline $\mathrm{K}_{2} \mathrm{O} \ldots \ldots$ & & & .10 \\
\hline $\mathrm{Na}_{22} \mathrm{O}$ & - . & $\ldots$ & .17 \\
\hline $\mathrm{H}_{2} \mathrm{O} \ldots$ & 4.37 & 2.45 & 2.50 \\
\hline $\mathrm{Cl}$ & & .04 & .07 \\
\hline & 99.82 & 100.07 & 98.99 \\
\hline
\end{tabular}




\section{CARNOTITE.}

From Montrose County, Colo. See memoir by Hillebrand and Ransome, Am. Jour. Sci., 4th ser., vol. 10, p. 120.

A, B, C. From Copper Prince claim, Roc Creek.

D, E. From Yellow Boy claim, La Sal Creek.

Analyses by W. F. Hillebrand.

\begin{tabular}{|c|c|c|c|c|c|}
\hline & A. & B. & C. & D. & E. \\
\hline $\mathrm{V}_{2} \mathrm{O}_{5-}$ & 18.35 & 18.49 & 15.76 & 17.80 & 18.05 \\
\hline $\mathrm{As}_{2} \mathrm{O}_{5} \ldots$ & .25 & trace & none & none & none \\
\hline $\mathrm{P}_{2} \mathrm{O}_{5} \ldots$ & .33 & .80 & .40 & trace & .05 \\
\hline $\mathrm{SiO}_{2}$ & $\ldots$ & .15 & .13 & 5.05 & .20 \\
\hline $\mathrm{TiO}_{2} \ldots \ldots \ldots$ & .10 & .03 & (?) & $\ldots \ldots$ & (?) \\
\hline $\mathrm{CO}_{2} \ldots \ldots \ldots$ & .33 & ' .56 & none & & none \\
\hline $\mathrm{SO}_{3} \ldots \ldots \ldots$ & .12 & $\ldots$ & .18 & $\cdots$ & none \\
\hline $\mathrm{UO}_{3} \ldots \ldots \ldots$ & 52.25 & 54.89 & 47.42 & 52.28 & 54.00 \\
\hline $\mathrm{MoO}_{3} \ldots \ldots$ & .23 & .18 & .18 & undet. & .05 \\
\hline $\mathrm{CrO}_{3}$ & trace & $\cdots$ & $\cdots$ & $\ldots$ & \\
\hline $\mathrm{Fe}_{2} \mathrm{O}_{3} \ldots \ldots \ldots$ & 1.77 & .21 & .72 & 3.36 & .42 \\
\hline $\mathrm{Al}_{2} \mathrm{O}_{3}$ & 1.08 & .09 & .08 & undet. & .29 \\
\hline $\mathrm{PbO} \ldots$ & .25 & .13 & .18 & undet. & .07 \\
\hline $\mathrm{CuO} \ldots \ldots$ & .20 & .15 & .22 & $\ldots$ & trace \\
\hline $\mathrm{CaO} \ldots \ldots$. & 2.85 & 3.34 & 2.57 & 1.85 & 1.86 \\
\hline $\mathrm{SrO}$ & $\ldots$ & .02 & $(?)$ & $\cdots$ & trace \\
\hline $\mathrm{BaO} \ldots . . .$. & .72 & .90 & .65 & 3.21 & 2.83 \\
\hline $\mathrm{MgO} \ldots \ldots$ & .20 & .22 & .24 & .17 & .14 \\
\hline $\mathrm{Li}_{2} \mathrm{O}_{\ldots} \ldots \ldots$ & none & trace & $(?)$ & trace & trace \\
\hline $\mathrm{Na}_{2} \mathrm{O}$ & .09 & .14 & .07 & .07 & .13 \\
\hline $\mathrm{K}_{2} \mathrm{O} \ldots \ldots$ & 6.73 & 6.52 & 6.57 & 5.32 & 5.46 \\
\hline $\mathrm{H}_{2} \mathrm{O}$ at $105^{\circ} \ldots$ & 2.59 & 2.43 & 1.85 & 4.52 & 3.16 \\
\hline $\mathrm{H}_{2} \mathrm{O}$ above $105^{\circ}$ & 3.06 & 2.11 & 2.79 & 3.87 & 2.21 \\
\hline \multirow[t]{2}{*}{ Insoluble $\ldots \ldots \ldots$} & 8.34 & 7.10 & 19.00 & & 10.33 \\
\hline & 99.84 & 98.46 & 99.01 & 97.50 & 99.25 \\
\hline
\end{tabular}




\section{ARSENATES AND ANTIMONATES.}

OLIVENITE.

From the American Eagle mine, Tintic mining district, Utah. Described by. Hillebrand in Bulletin 20. Analysis by W. F. Hillebrand.

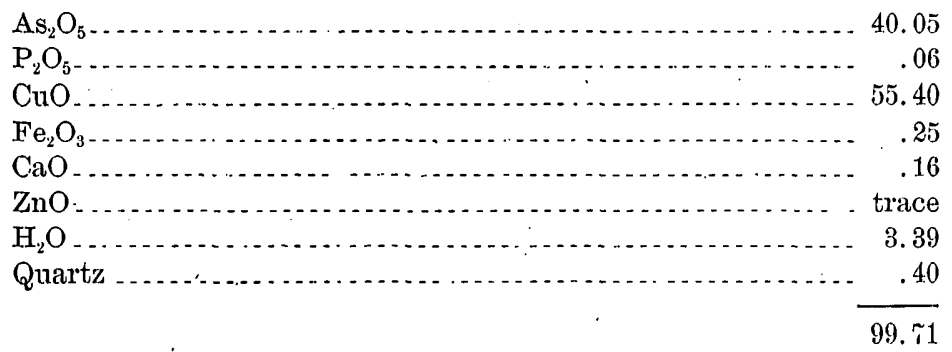

ERINITE.

From the Mammoth mine, Tintic district, Utah. Described by Hillebrand and Washington in Bulletin 55. Analyses by W. F. Hillebrand.

\begin{tabular}{|c|c|c|}
\hline & A. & B. \\
\hline $\mathrm{As}_{2} \mathrm{O}_{5}$ & 33.53 & 31.91 \\
\hline $\mathrm{P}_{2} \mathrm{O}_{5}$ & .10 & ..... \\
\hline $\mathrm{CuO} \ldots \ldots$ & 57.67 & 57.51 \\
\hline $\mathrm{ZnO} \ldots \ldots \ldots$ & 1.06 & .59 \\
\hline $\mathrm{CaO}-\ldots \ldots$ & .32 & .51 \\
\hline $\mathrm{MgO} \ldots$ & trace & trace \\
\hline $\mathrm{Fe}_{2} \mathrm{O}_{3}$ & .14 & .20 \\
\hline \multirow{2}{*}{$\mathrm{H}_{2} \mathrm{O} \ldots \ldots$} & 7.22 & 9.15 \\
\hline & 100.04 & 99.87 \\
\hline
\end{tabular}

CLINOCLASITE.

From the Mammoth mine, Tintic district, Utah. Described by Hillebrand and Washington in Bulletin 55. Analyses by W. F. Hillebrand. Specific gravity, 4.38 at $19^{\circ}$.

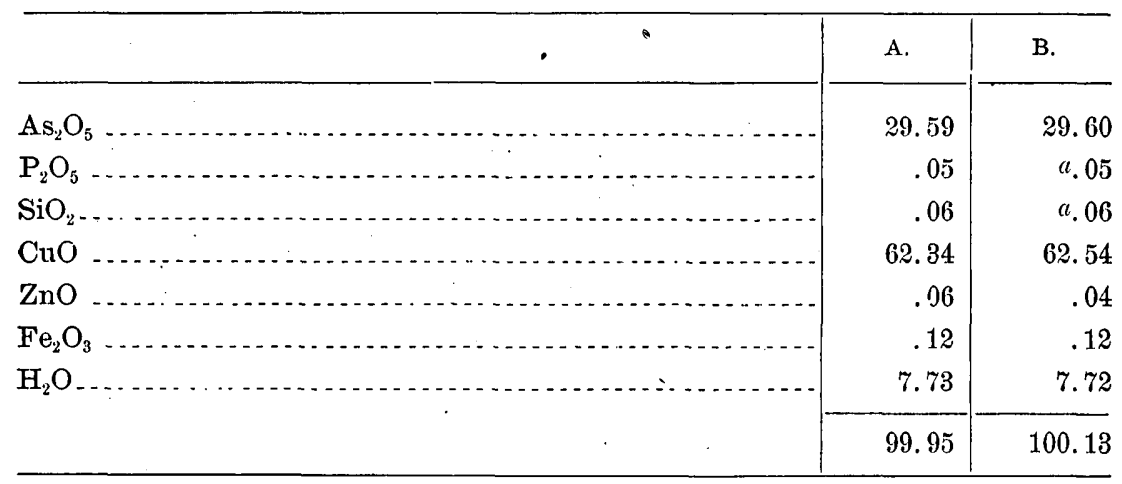

"Assumed the same as in A. 
CONICHALCITE.

From the American Eagle mine, Tintic district, Utah. Analysis by W. F. Hillebrand, with description in Bulletin.20.

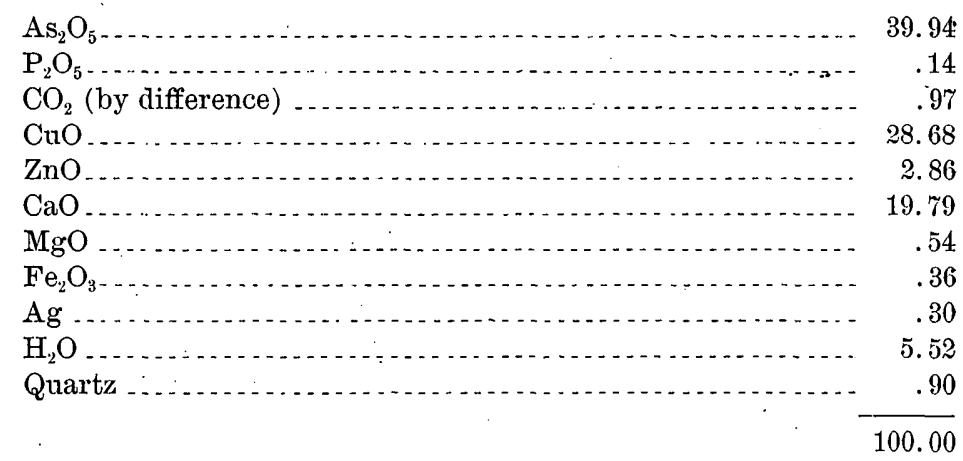

TYROLITE.

From the Mammoth mine, Tintic district, Utah. Described by Hillebrand and Washington in Bulletin 55. Analyses by W: F. Hillebrand. Specific gravity, 3.27 at $20^{\circ} .5$.

\begin{tabular}{|c|c|c|}
\hline & A. & B. \\
\hline $\mathrm{As}_{2} \mathrm{O}_{5}$ & 28.78 & 26.22 \\
\hline $\mathrm{P}_{2} \mathrm{O}_{5} \ldots \ldots \ldots \ldots$ & trace & trace \\
\hline $\mathrm{CuO} \ldots .$. & 45.22 & 46.38 \\
\hline $\mathrm{ZnO} \ldots$ & .04 & trace \\
\hline $\mathrm{CaO} \ldots$ & 6.84 & 6.69 \\
\hline $\mathrm{MgO} \ldots$ & .05 & .04 \\
\hline (2. & $\cdot 17.26$ & 17.57 \\
\hline \multirow[t]{2}{*}{$\mathrm{SO}_{3}$} & $(?)$ & 2.27 \\
\hline & 98.19 & 99.17 \\
\hline
\end{tabular}

\section{CHENEVIXITE.}

From the American Eagle mine, Tintic district, Utah. Analyzed by W. F. Hillebrand and described in Bulletin 20.

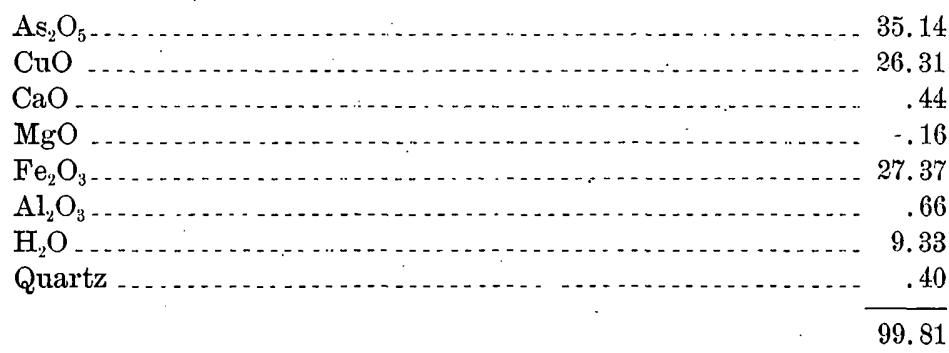


MIXITE.

From the Mammoth mine, Tintic district, Utah. Described by Hillebrand and Washington in Bulletin 55. Analysis by W. F. Hillebrand.

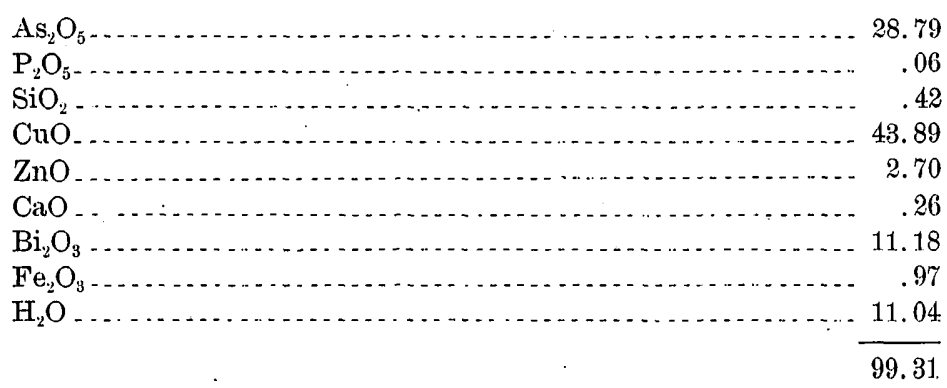

SCORODITL.

An incrustation on hot spring deposits, from Joseph's Coat Spring, Broad Creek, Yellowstone Park. Analyzed by J. E. Whitfield and described in Bulletin õ.

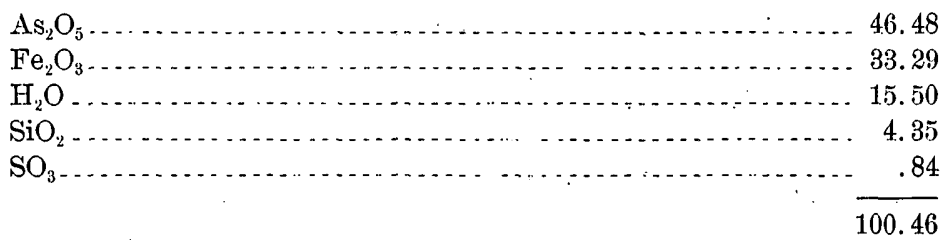

BINDHEIMITE.

From a claim near the Bertrand mine, Secret Canyon; Nevada. Analyzed by W. F. Hillebrand and described in Bulletin 20. Specific gravity, 5.01 at $19^{\circ}$, after correction for admixed quartz and cerussite.

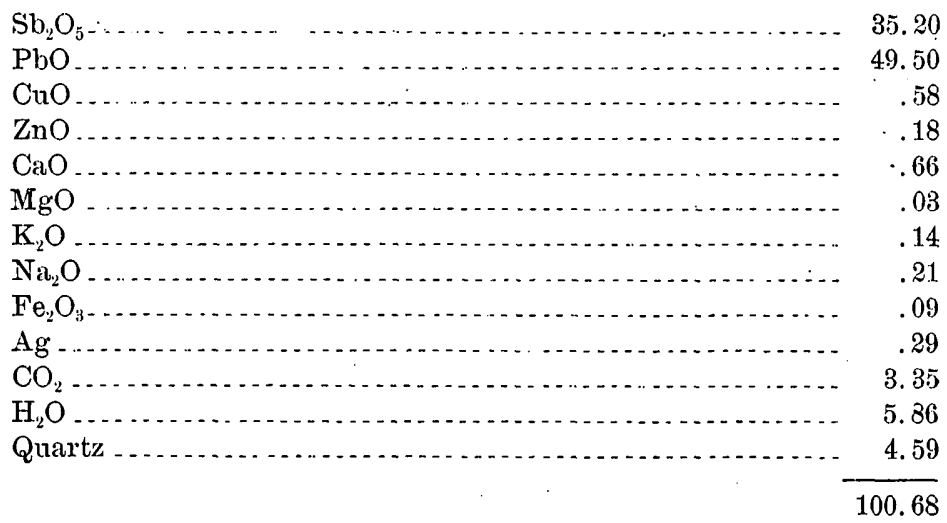




\section{SULPHATES AND TELLURITES.}

GYPSUM.

A. From Hillsboro, New Brunswick. Analysis by George, Steiger.

B. From the Western Plaster Works, Alabaster, Mich. Analysis by George Steiger.

C. From east of Cascade, Black Hills, South Dakota. Analysis by George Steiger.

D. From Rico-Aspen mine, Rico district, Colorado. Analysis by W. F. Hillebrand.

E, F. From Nephi, Utah. Analyses by E. T. Allen. Some anhydrite must be present.

\begin{tabular}{|c|c|c|c|c|c|c|}
\hline & A. & B. & C. & D. & E. & F. \\
\hline $\mathrm{SO}_{3} \ldots$ & 46.18 & 46.18 & 45.45 & 45.07 & 48.14 & 39.53 \\
\hline $\mathrm{CO}_{2 \ldots} \ldots$ & $\ldots$ & $\ldots$ & .85 & 1.54 & .65 & 7.73 \\
\hline $\mathrm{Cl} \ldots$ & trace & .03 & $\cdots$ & $\ldots$ & trace & .04 \\
\hline $\mathrm{SiO}_{2}$ & & & $\cdot .10$ & .51 & & \\
\hline $\mathrm{TiO}_{2}$ & & & ..... & trace & & - \\
\hline $\mathrm{Al}_{2} \mathrm{O}_{3}-$ & & & .12 & .03 & & 14 \\
\hline $\mathrm{Fe}_{2} \mathrm{O}_{3} \ldots \ldots$ & .10 & .00 & $-\cdots$ & .09 & - - - & .14 \\
\hline $\mathrm{CaO} \ldots \ldots$. & 32.37 & 32.33 & 32.44 & 32.49 & 35.29 & 38.46 \\
\hline $\mathrm{SrO} \ldots .$. & $\ldots$ & $\ldots$ & $\ldots \ldots$ & .10 & $\ldots$ & $\ldots$ \\
\hline $\mathrm{MgO} \ldots$ & trace & .05 & .33 & .92 & trace & .24 \\
\hline $\mathrm{Na}_{2} \mathrm{O} \ldots$ & .10 & .14 & & trace & & .07 \\
\hline $\mathrm{K}_{2} \mathrm{O} \ldots \ldots$ & & & & 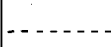 & $\ldots$. & .19 \\
\hline $\mathrm{H}_{2} \mathrm{O} \ldots \ldots \ldots$ & 20.94 & 20.96 & 20.80 & 19.67 & 15.88 & 12.69 \\
\hline Insoluble ....... & .10 & .05 & & $\ldots$ & & .45 \\
\hline Organic matter...... & & & (....... & present & & \\
\hline & 99.79 & 99.82 & 100.09 & 100.42 & 99.96 & 99.54 \\
\hline
\end{tabular}

BROCHANTITE.

From the United Verde mine, Jerome, Ariz. Analysis by W. F. Hillebrand.

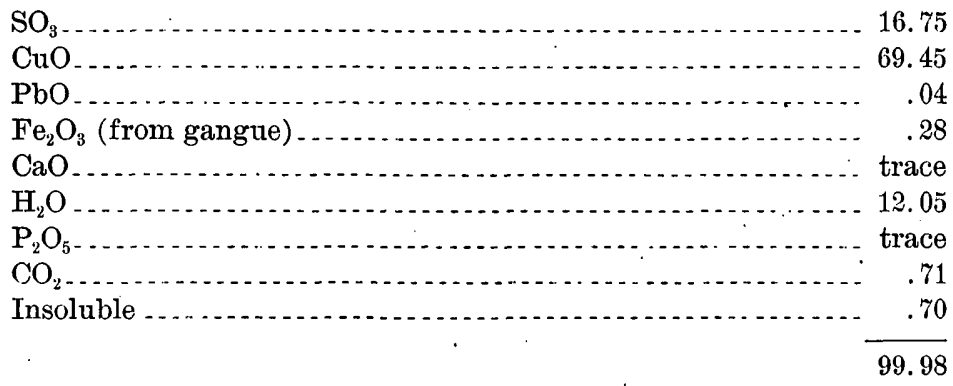


ANTLERITE.

From the Antler mine, Yucca Station, Mohave County, Ariz. Described by Hillebrand as a new species in Bulletin 5̃. Specific gravity, 3.93 at $16^{\circ}$, corrected for gangue. Analyses by W. F. Hillebrand.

A, B. First lot received.

C. Later sample.

\begin{tabular}{|c|c|c|c|}
\hline & A. & B. & C. \\
\hline $\mathrm{SO}_{3}$ & 18.78 & 18.48 & 20.11 \\
\hline $\mathrm{CuO} \ldots \ldots$ & 62.48 & 62.69 & 63.26 \\
\hline $\mathrm{ZnO} \ldots \ldots$. & .27 & .27 & .04 \\
\hline $\mathrm{CaO} \ldots \ldots . .$. & .06 & .05 & .04 \\
\hline $\mathrm{MgO} \ldots \ldots . . . . . . .$. & .03 & .03 & 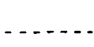 \\
\hline $\mathrm{Al}_{2} \mathrm{O}_{3}, \mathrm{Fe}_{2} \mathrm{O}_{3} \ldots$ & .14 & .12 & .15 \\
\hline $\mathrm{SiO}_{2} \ldots \ldots \ldots$ & .06 & undet. & .05 \\
\hline $\mathrm{H}_{2} \mathrm{O}$ & - 10.21 & 10.18 & 10.05 \\
\hline \multirow[t]{2}{*}{ Insoluble gangue .. } & 8.01 & 8.02 & 6.27 \\
\hline & 100.04 & 99.84 & 99.97 \\
\hline
\end{tabular}

PISANITE.

From Bingham, Utah. Analysis by W. F. Hillebrand.

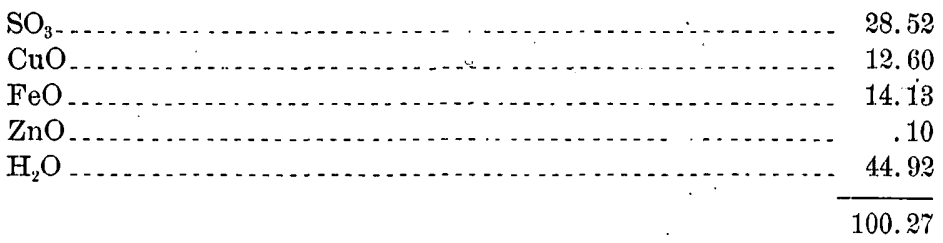

PICRALLUMOGENE.

From near Las Vegas, N. Mex. Analysis by W. F. Hillebrand.

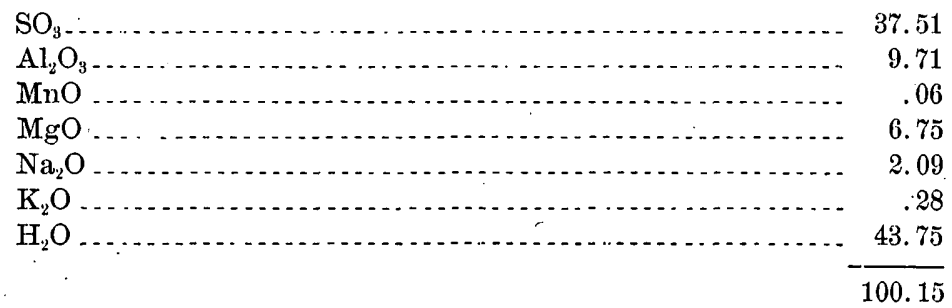




\section{HALOTRICHITE.}

From the headwaters of Gila River, Grant County, N. Mex., about 40 miles north of Silver City. Analyzed by F. W. Clarke, and described in Bulletin 9 .

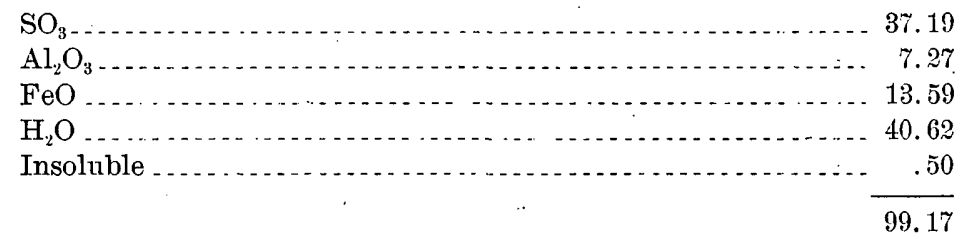

ALUNOGEN.

A. From headwaters of Gila River, Grant County, N. Mex., about 40 miles north of Silver City. Analyzed by F.W. Clarke, and described in Bulletin 9. Color, pinkish.

B. From the calcite spring, Yellowstone Park. Fine, white, silky fibers. Analysis by J. E. Whitfield.

C. From the Grand Canyon, Yellowstone Park. Analysis by J. E. Whittield.

\begin{tabular}{|c|c|c|c|}
\hline & A. & B. & C. \\
\hline $\mathrm{SO}_{3} \ldots$ & 34.43 & 40.65 & 38.22 \\
\hline $\mathrm{Al}_{2} \mathrm{O}_{3}$ & 15.52 & 15.72 & 16.80 \\
\hline $\mathrm{Fe}_{2} \mathrm{O}_{3} \ldots \ldots$ & & trace & trace \\
\hline $\mathrm{CaO} \ldots \ldots$ & ...... & trace & none \\
\hline $\mathrm{MgO} \ldots$ & .. & 1.53 & .18 \\
\hline $\mathrm{K}_{2} \mathrm{O} \ldots$ & & . & .01 \\
\hline $\mathrm{Na}_{2} \mathrm{O}_{-}$ & 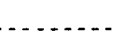 & $\ldots$ & .63 \\
\hline $\mathrm{H}_{2} \mathrm{O}_{-.-}$ & 42.56 & 41.81 & 43.64 \\
\hline $\mathrm{SiO}_{2 \ldots} \ldots \ldots$ & - ....... & .09 & 1.38 \\
\hline \multirow[t]{2}{*}{ Insoluble... } & 7.62 & & (............ \\
\hline & 100.13 & 99.80 & 100.86 \\
\hline
\end{tabular}

COPIAPITE.

A. From the Redington mine, Knoxville, Cal.

B. From Sulphur Bank, Lake County, Cal.

Analyses by W. H. Melville. Description by Melville and Lindgren in Bulletin 61. 


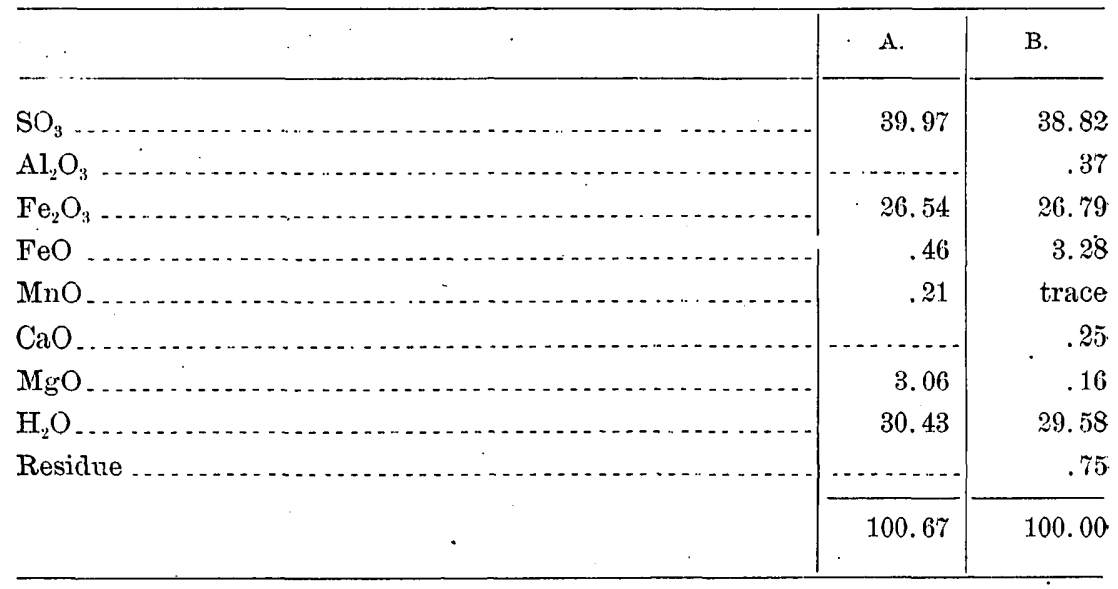

\section{KNOXVILLITE.}

From the Redington mine, Knoxville, Cal. Described as a new species by Melville and Lindgren in Bulletin 61 . Analysis by W. H. Melville.

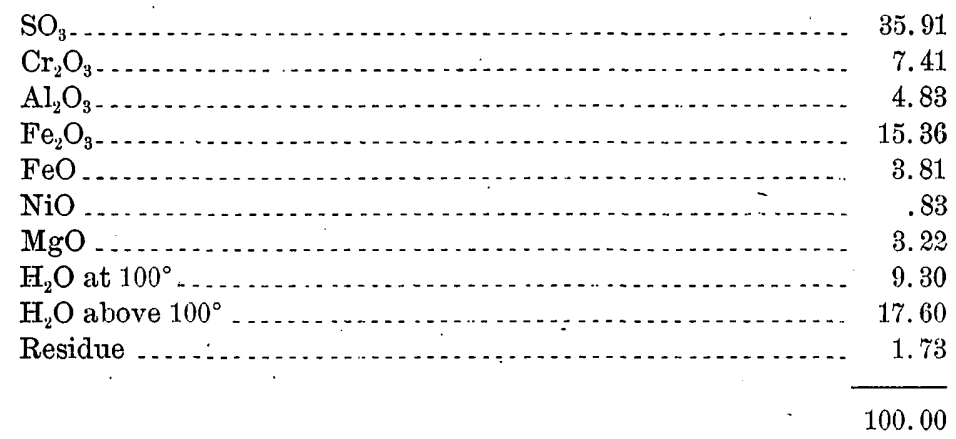

REDINGTONITE.

From the Redington mine, Knoxville, Cal. Described as a new species by Melville and Lindgren in Bulletin 61 . Analysis by W. H. Melville. Specific gravity, 1.761.

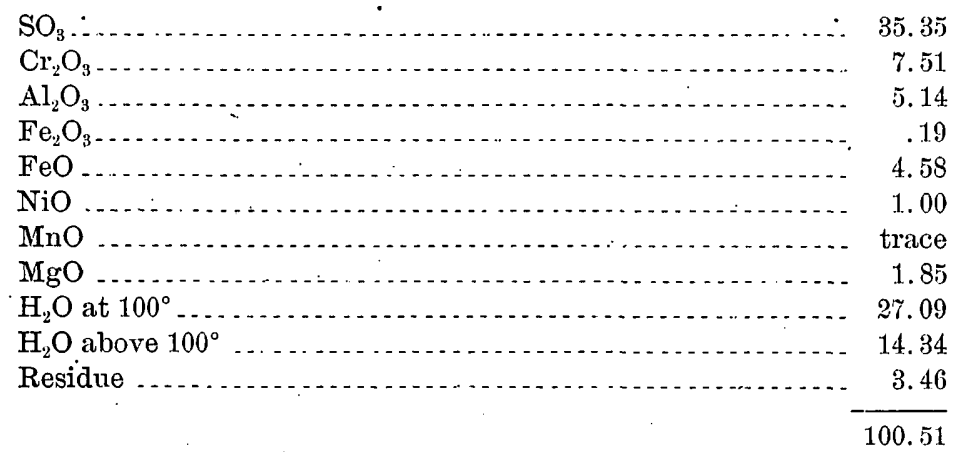


ALUNITE.

A. From Knickerbocker Hill, Custer County, Colo. Analysis by L. G. Eakins.

B. From Tres Cerritos Buttes, sonthwest of Indian Gulch, Mariposa County, Cal. Analysis by William Valentine.

\begin{tabular}{|c|c|c|}
\hline & A. & B. \\
\hline $\mathrm{SO}_{3} \ldots \ldots$ & 35.91 & 38.50 \\
\hline $\mathrm{Al}_{2} \mathrm{O}_{3}-\ldots \ldots \ldots$ & 38.91 & 38.05 \\
\hline $\mathrm{Fe}_{2} \mathrm{O}_{3} \ldots \ldots$ & $\ldots . .$. & .23 \\
\hline $\mathrm{CaO}$ & .35 & .55 \\
\hline MgO & trace & trace \\
\hline $\mathrm{K}_{2} \mathrm{O} \ldots \ldots$ & 4.03 & 4.48 \\
\hline $\mathrm{Na}_{2} \mathrm{O} \ldots \ldots \ldots$ & 4.32 & 2.78 \\
\hline $\mathrm{H}_{2} \mathrm{O}$ & 13.03 & 11.92 \\
\hline $\mathrm{SiO}_{2}$ & 2.82 & 2.64 \\
\hline $\mathrm{TiO}_{2} \ldots \ldots \ldots \ldots$ & ....... & .40 \\
\hline \multirow[t]{2}{*}{$\mathrm{P}_{2} \mathrm{O}_{5}-$} & ....... & trace \\
\hline & 99.37 & 99.55 \\
\hline
\end{tabular}

JAROSITE.

A. From Black Iron mine, Eagle County, Colo.

B. From Pigeon mine, Rico district, Colorado.

Analyses by W. F. Hillebrand.

\begin{tabular}{|c|c|c|}
\hline & A. & B. \\
\hline $\mathrm{SO}_{3} \ldots \ldots$ & 30.72 & 28.20 \\
\hline $\mathrm{Fe}_{2} \mathrm{O}_{3} \ldots \ldots \ldots$ & $46.37^{\circ}$ & 43.81 \\
\hline $\mathrm{PbO}$ & 1.80 & $\ldots . . .$. \\
\hline 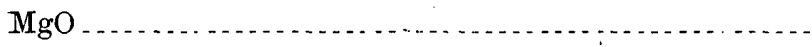 & trace & trace \\
\hline $\mathrm{CaO} \ldots \ldots . .$. & $-\ldots$. & .06 \\
\hline $\mathrm{K}_{2} \mathrm{O} \ldots \ldots \ldots \ldots$ & 8.14 & 7.44 \\
\hline $\mathrm{Na}_{2} \mathrm{O} \ldots \ldots \ldots$ & .27 & .08 \\
\hline $\mathrm{H}_{2} \mathrm{O} \ldots \ldots \ldots \ldots$ & 11.40 & 11.64 \\
\hline $\mathrm{Al}_{2} \mathrm{O}_{3} \ldots \ldots \ldots$ & & 1.00 \\
\hline $\mathrm{P}_{2} \mathrm{O}_{5}$ & -.. & .33 \\
\hline $\mathrm{As}_{2} \mathrm{O}_{5}$ & .51 & $\ldots$ \\
\hline \multirow[t]{2}{*}{$\mathrm{SiO}_{2} \ldots \ldots \ldots$} & .72 & 7.35 \\
\hline & 99.93 & 99.91 \\
\hline
\end{tabular}


NATROJAROSITE.

From Soda Springs Valley, on road from Sodaville to Vulcan copper mine, Nevada. Described by Hillebrand and Penfield in Am. Jour. Sci., 4th ser., vol. 14, p. 211. Analysis by W. F. Hillebrand. Specific gravity, 3.18 at $30.5^{\circ}$.

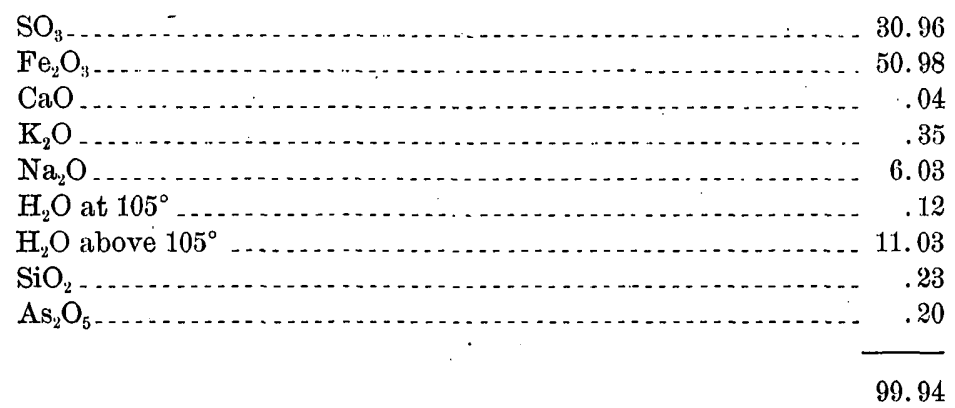

PLUMBOJAROSITE.

From Cooks Peak, New Mexico. Described as a new species by Hillebrand and Penfield in Am. Jour. Sci., 4th ser., vol. 14, p. 211. Analysis by W. F. Hillebrand. Specific gravity, 3.668 at $30.5^{\circ}$.

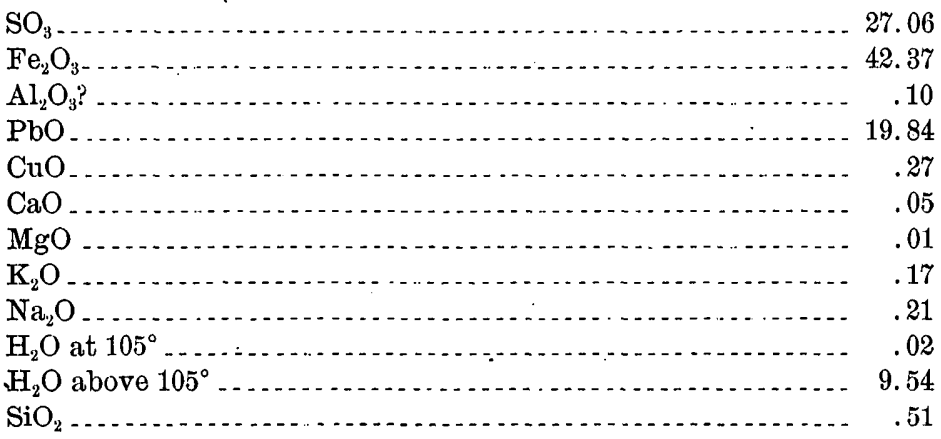

100.15 
EMMONSITE.

A hydrous ferric tellurite from near Tombstone, Ariz. Analyzed partially by W. F. Hillebrand, and described by him as a new species in Proc. Colorado Sci. Soc., vol. 2, pt. 1, p. 21. The data relative to the coinposition of emmonsite are as follows:

\begin{tabular}{|c|c|c|c|c|}
\hline & I. & II. & III: & IV. \\
\hline $\mathrm{Te}(\mathrm{Se}) \ldots \ldots$ & $59.7 \%$ & 59.15 & 59.05 & 59.14 \\
\hline $\mathrm{Fe} \ldots . .$. & 14.00 & 14.06 & 14.90 & 14.20 \\
\hline $\mathrm{ZnO} \ldots$ & $\ldots \ldots$ & & & 1.94 \\
\hline $\mathrm{CaO} \ldots$ & & & & .56 \\
\hline $\mathrm{H}_{2} \mathrm{O} \ldots$ & 3.28 & & & \\
\hline
\end{tabular}

XVI. MOLYBDATES, TUNGSTATES, AND URANATES.

POWELLTTE.

From the Seven Devils mining district, Idaho. Analyzed by W. H. Melville, and described by him as a new mineral species in Bulletin 90. Specific gravity, 4.526 .

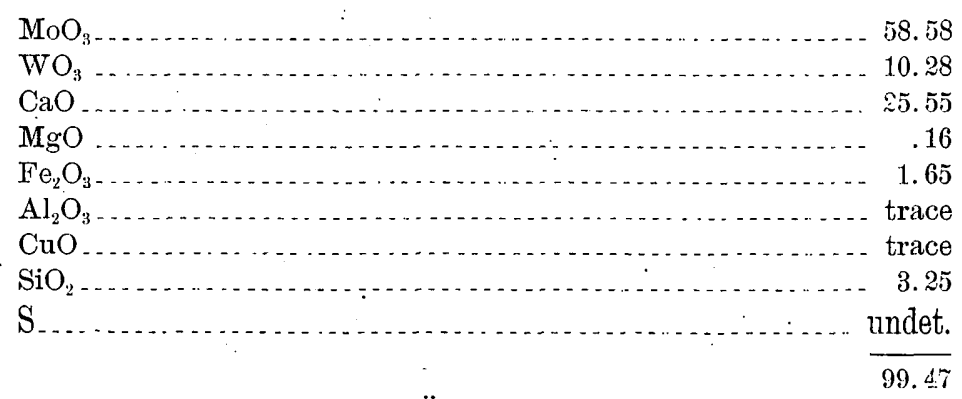

HÜBNERITE.

From the Royal Albert vein, Uncompahgre district, Ouray County, Colo. Analyzed by W. F. Hillebrand, and described by him in Bulletin 20. Specific gravity, 7.177 at $24^{\circ}$.

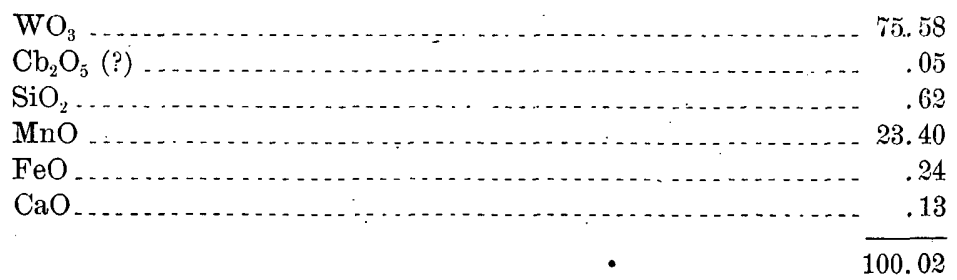


URANINITE.

Analyses by W. F. Hillebrand. Discussed in Bulletins 78 and 90 . In the original publications the gaseous constituent of uraninite was supposed to be nitrogen, as indeed it was in part. The discovery of helium, however, has shed new light upon the subject, and the analyses have been corrected accordingly,

A, B, C, D, E. Crystallized uraninite from Hale's quarry, Glastonbury, Conn. Nitrogen was certainly present in these specimens.

\begin{tabular}{|c|c|c|c|c|c|}
\hline & A. & B. & C. & D. & E. \\
\hline $\mathrm{UO}_{3-}$ & 22.08 & 23.35 & 22.22 & 26.48 & 23.03 \\
\hline $\mathrm{UO}_{2} \ldots \ldots$ & 59.13 & 58.01 & 59.31 & 57.43 & 59.93 \\
\hline $\mathrm{ThO}_{2} \ldots \ldots$ & & 978 & & 9.79 & \\
\hline $\mathrm{CeO}_{2} \ldots$ & & 0.10 & & .25 & \\
\hline $\mathrm{ZrO}_{2} ? \ldots$ & 9.57 & & 10.31 & - $\ldots \ldots$ & 11.10 \\
\hline$(\mathrm{LaDi})_{2} \mathrm{O}_{3}$ & & & & .13 & \\
\hline$(\mathrm{YEr})_{2} \mathrm{O}_{3} \ldots$ & & 6 & & .20 & \\
\hline $\mathrm{PbO}$ & 3.14 & 3.24 & 3.07 & 3.26 & 3.08 \\
\hline $\mathrm{CaO} \ldots$ & .08 & & undet. & .08 & .11 \\
\hline $\mathrm{MgO}_{-}$ & $\ldots$ & & undet. & ...... & $\ldots$ \\
\hline Alkalies : & $\ldots \ldots$ & - - - - - - & undet. & trace & $\ldots$ \\
\hline $\mathrm{H}_{2} \mathrm{O} \ldots \ldots$ & .97 & undet. & undet. & .61 & .43 \\
\hline He, etc... & undet. & undet. & undet. & undet. & .34 \\
\hline $\mathrm{Fe}_{22} \mathrm{O}_{3} \ldots \ldots$ & 1.21 & .33 & 67. & .40 & .29 \\
\hline $\mathrm{MnO}$ & -...... & $\ldots$ & $\ldots$. & trace & $\ldots$ \\
\hline $\mathrm{SiO}_{2} \ldots \ldots$ & 1.06 & & .25 & .16 & .16 \\
\hline $\mathrm{P}_{2} \mathrm{O}_{5}$ & & & & & .02 \\
\hline $\mathrm{F}$ & - - & $\ldots$ & $-\ldots$ & $\ldots$ & .04 \\
\hline Insoluble $\ldots . . . . . . . . . .$. & .85 & 1.74 & .42 & .70 & .89 \\
\hline \multirow[t]{2}{*}{$\mathrm{Cb}_{2} \mathrm{O}_{5} \ldots \ldots$} & .96 & & & $\ldots \ldots \ldots$ & (......... \\
\hline & 99.05 & 96.91 & 96.25 & 99.49 & 99.42 \\
\hline Specific gravity & 9.139 & 9.051 & & 9.587 & 9.622 \\
\hline
\end{tabular}


F, G, H. Brilliantly crystallized, from Branchville, Conn.

I, J. Altered tiraninite, from the Flat Rock mine, Mitchell County, N. C.

K. Massive, from near Blackhawk, Colo.

L. From Marietta, S. C.

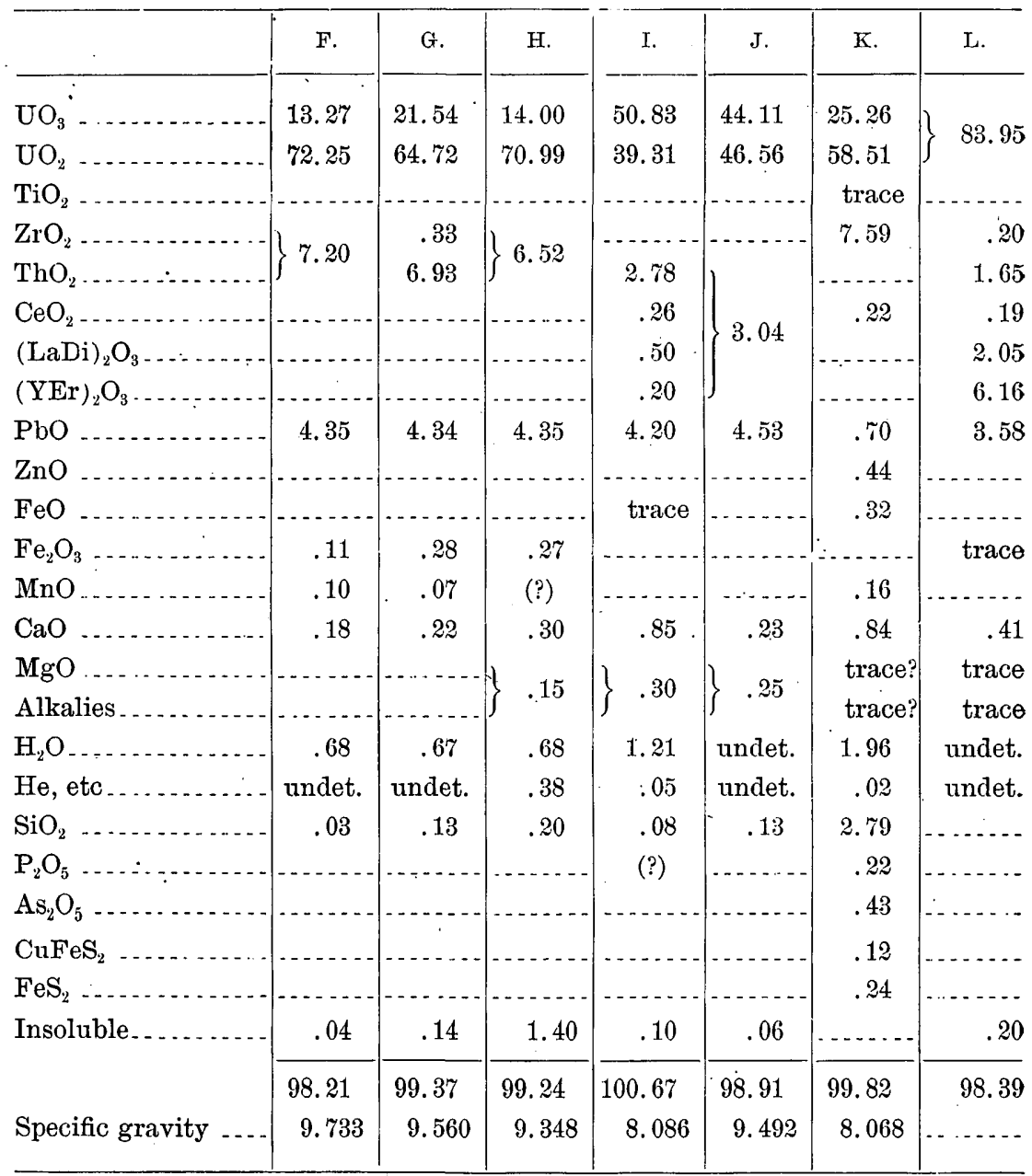


M. Nivenite from Llano County, Tex.

$\mathrm{N}$. Somewhat altered uraninite from Villeneuve, Province of Quebec, Canada.

O. Massive uraninite from Johanngeorgenstadt, Saxony.

\begin{tabular}{|c|c|c|c|}
\hline & M. & $\mathrm{N}$ & o: \\
\hline $\mathrm{UO}_{3} \ldots$ & 20.89 & 34.67 & 22.33 \\
\hline $\mathrm{UO}_{2, \ldots \ldots \ldots \ldots}$ & 44.17 & 41.06 & 59.30 \\
\hline $\mathrm{ThO}_{2} \ldots \ldots$ & 6.69 & 6.41 & \\
\hline $\mathrm{ZrO}_{2}$ & .34 & $(?)$ & \\
\hline $\mathrm{CeO}_{2}$ & .34 & .40 & none \\
\hline$(\mathrm{La}, \mathrm{Di})_{2} \mathrm{O}_{3} \ldots \ldots$ & 2.36 & 1.11 & \\
\hline$(\mathrm{Y}, \mathrm{Er})_{2} \mathrm{O}_{3} \ldots \ldots$ & 9.46 & 2.57 & \\
\hline $\mathrm{CaO} \ldots$ & .32 & .39 & 1.00 \\
\hline $\mathrm{PbO} \ldots .$. & 10.08 & 11.27 & 6.39 \\
\hline $\mathrm{H}_{2} \mathrm{O} \ldots \ldots \ldots$ & 1.48 & 1.47 & 3.17 \\
\hline He, etc ............ & .08 & .10 & trace \\
\hline $\mathrm{SiO}_{2} \ldots$ & .46 & .19 & .50 \\
\hline $\mathrm{Fe}_{2} \mathrm{O}_{3} \ldots \ldots \ldots$ & .14 & .10 & .21 \\
\hline $\mathrm{Al}_{2} \mathrm{O}_{3} ? \ldots \ldots$ & & $\ldots$ & .20 \\
\hline $\mathrm{MgO} \ldots \ldots \ldots \ldots \ldots$ & & & .17 \\
\hline $\mathrm{Na}_{2} \mathrm{O}$ & & & .31 \\
\hline $\mathrm{Bi}_{2} \mathrm{O}_{3} \ldots \ldots$ & $\ldots$ & .09 & .75 \\
\hline $\mathrm{CuO} \ldots \ldots \ldots$ & & & .17 \\
\hline $\mathrm{MnO} \ldots \ldots \ldots$ & & & .09 \\
\hline $\mathrm{P}_{2} \mathrm{O}_{5} \ldots \ldots$ & 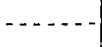 & 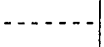 & .06 \\
\hline $\mathrm{As}_{2} \mathrm{O}_{5} \ldots \ldots \ldots$ & . & $\cdots$ & 2.34 \\
\hline $\mathrm{V}_{2} \mathrm{O}_{5}, \mathrm{MoO}_{3}, \mathrm{WO}_{3} \mathrm{i}$ & & $\ldots$ & .75 \\
\hline $\mathrm{SO}_{3} \ldots \ldots \ldots$ & . . . & & .19 \\
\hline \multirow[t]{2}{*}{ Insoluble. . } & 1.47 & .13 & \\
\hline & 98.28 & 99.96 & 97.93 \\
\hline Specific gravity ..... & 8.29 & $\cdots$ & 6.89 \\
\hline
\end{tabular}

Bull. $220-03 \longrightarrow 8$ 
The following uraninites are all from Norway, and were analyzed for comparison with the American material.

P. Bröggerite from Anneröd, near Moss. Specific gravity, 8.893.

Q. From Elvestad. Specific gravity, 9.145.

R. From Elvestad. Specific gravity, 8.320.

S. From Skraatorp. Specific gravity, 8.966.

T. From Huggenäskilen. Specific gravity, 8.930.

U. Cleveite from Arendal. Specific gravity, 7.500.

V. From Arendal.

\begin{tabular}{|c|c|c|c|c|c|c|c|}
\hline & P. & Q. & $\mathrm{R}$. & s. & $\mathrm{T}$. & U. & V. \\
\hline $\mathrm{UO}_{3}$. & 30.63 & 25.36 & 22.04 & 32.00 . & 35.54 & 41.71 & 26.80 \\
\hline $\mathrm{UO}_{2} \ldots$ & 46.13 & 50.74 & 43.03 & 43.88 & 43.38 & 24.18 & 44.18 \\
\hline $\mathrm{ZrO}_{2} ?$ & .06 & .08 & & $\ldots \ldots$ & $\therefore$ & & $\ldots$ \\
\hline $\mathrm{ThO}_{2} \ldots \ldots \ldots$ & 6.00 & 8.48 & & 8.98 & 6.63 & 3.66 & 4.15 \\
\hline $\mathrm{CeO}_{2} \ldots \ldots$ & .18 & .21 & 8.43 & .17 & .20 & & none \\
\hline$(\mathrm{La}, \mathrm{Di})_{2} \mathrm{O}_{3} \ldots$ & .27 & .26 & & .36 & .23 & & .67 \\
\hline$(\mathrm{Y}, \mathrm{Er})_{2} \mathrm{O}_{3} \ldots$ & 1.11 & 1.10 & & .97 & - T. 03 & 9.76 & 9.05 \\
\hline $\mathrm{PbO} \ldots$ & 9.04 & 10.06 & 8.58 & 9.46 & 9.44 & 10.54 & 10.95 \\
\hline $\mathrm{CaO} \ldots$ & .37 & .77 & .37 & .36 & .41 & 1.06 & .61 \\
\hline $\mathrm{MgO}$. & traces & traces & 13 & traces & 13 & .10 & .04 \\
\hline Alkalies . & & & .10 & Hides & .10 & .23 & .15 \\
\hline $\mathrm{H}_{2} \mathrm{O} \ldots \ldots$ & .74 & .73 & .74 & .77 & .79 & 1.23 & undet. \\
\hline $\mathrm{He}$, etc $\ldots . . .$. & .17 & .18 & .15 & .15 & .15 & undet. & .18 \\
\hline $\mathrm{Fe}_{2} \mathrm{O}_{3} \ldots$ & .25 & .21 & .30 & .09 & .32 & .03 & .24 \\
\hline $\mathrm{MnO} \ldots$ & $\ldots$ & .06 & & & & & - . \\
\hline $\mathrm{SiO}_{2} \ldots \ldots \ldots$ & .22 & .38 & .29 & .53 & .49 & .90 & .50 \\
\hline $\mathrm{P}_{2} \mathrm{O}_{5}$ & .02 & .04 & trace & $(?)$ & trace & & trace \\
\hline \multirow[t]{2}{*}{ Insoluble. . } & 4.42 & .45 & 15.45 & 1.54 & .42 & 1.10 & 1.19 \\
\hline & 99.61 & 99.11 & 99.51 & 99.26 & 99.16 & 94.50 & 98.71 \\
\hline
\end{tabular}




\section{N D R X}

Actinolite

Egirite.

Aiken,.S. C

Alabaster, Mich

Albite.

Allanite .

Almandite

Alpine County, Cal

Alunite.

Alunogen.

Amador County, Cal........... . .

Amelia Court-House, Va .........

Amphibole $37,40,41$

Analcite

Andradite

Anneröd, Norway

Annite

Anorthite

Anorthoclase

Anthracite Creek, Colo

Antlerite

Apatite.

Apophyllite

Arendal, Norway

Ashland County, Wis

Astrophyllite

Auburn, $\mathrm{Me}$

Augite

Axinite

Bakersville, N. C

Bald Mountain District, Mont

Baltimore, Md

Barkevikite

Bastnäsite

Banxite

Bear River, Cal

Bear Valley, Cal ...............

Bedford County, Va

Bergen Hill, N. J

Berufiörd, Iceland..

Beryl

Big Goose Canyon, Wyo

Big Trees quadrangle, Cal.
Page.

37

34

90

104

26

51.

45

75

108

106

75

46

41.

68

114

114

97

26

65

105

105

98

114

114

93

93

73,74

33,34

57

28

98

26,77

41

24

21

75

72

93

$6,57,68$

65

42

89

41
Bindheimite ................. 103

Bingham, Utah............... 105

Biotite .................. 74, 75, 80

Birch Lake, Minn. ............. $47^{\circ}$

Bismuthinite . ................ 11

Black Hawk, Colo ... . . . . . . . . . 112

Blandford, Mass ............... 84

Bloods Station, Cal . . . . . . . . . 75

Bole .......................... 91

Boltonite . . . . . . . . .

Boulder County, Colo........... 33

Bourg d'Oisans, Dauphiny ...... 57

Bozeman, Mont. ............... 34

Branchville, Conn ............ 112

Brindletown, N. C : . . . . . . .... 97

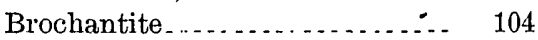

Bröggerite.................... 114

Broken Hill, Australia .......... 17

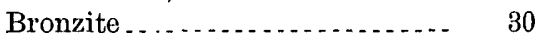

Brucite ..................... 22

Buck Creek, N. C............... 87

Buffalo Peaks, Colo . ............ 31

Burgess, Canada . . . . . . . . . . . . 76

Butte, Mont . . . . . . . . . . 11, 13, 41, 75

Calamine _................... 53

Calaveras County, Cal ........ 16, 17

Calaverite ..... . . . . . . . . . . 16

Calhao, Brazil . . . . . . . . . . . . 59, 62

Calico, Cal _... . . . . . . . . . 12

Canaan Mountain, Conn......... 37

Cancrinite.................... 43

Cape Prince of Wales, Alaska ... $\quad 38$

Carnotite . . . . . . . . . . . ....... 100

Carson Hill, Cal ... . . . . . . . . . . . . 17

Cascade, S. Dak ........... . . . 104

Chabazite .................. $\quad 67$

Chalk Mountain, Colo.......... 25

Chenevixite ................. 102

Chester, Mass ................ 85

Cheyenne Mountain, Colo ....... 24

Chlorite .................... 83

Chloritoid .................. 79

Chromite ................... 21 


\begin{tabular}{|c|c|c|c|}
\hline Chrysolite. & age. & Elvestad, Norway & $\begin{array}{l}\text { Page. } \\
114\end{array}$ \\
\hline Chrysotile . & 86 & Embolite . . . . . . . & 17 \\
\hline Cimolite.... & 91 & Emmonsite . . & 110 \\
\hline Cleveite. & 114 & Enargite .... & 13 \\
\hline Clifton, Ariz . & 46 & Enstatite ......... & 30 \\
\hline Clinoclasite. & 101 & Epidote ....... & 50 \\
\hline Clinochlore .................. & 83 & Erinite $\ldots . . . . . .$. & 101 \\
\hline Clip, Ariz .......... & 2,58 & Etchison, $\mathrm{Md} \ldots \ldots \ldots \ldots \ldots$ & 63,72 \\
\hline Cockeysville, Md. & 23 & Fassa, Tyrol $\ldots \ldots \ldots$ & 51 \\
\hline Colemanite ..... & 95 & Feldspar $\ldots \ldots \ldots \ldots$ & $, 28,29$ \\
\hline Colfax, Cal & 63 & Fibrolite . . . . . & 52 \\
\hline alcite ... & 102 & Florissant, Colo ........ & 52 \\
\hline Cooks Peak, N.Mex .......... & 109 & Fort Ellis, Mont . . . . . . . . . . . & 34 \\
\hline Copiapite....... & 106 & Fort Wingate, N. Mex ........ & 47 \\
\hline Cornwall, England ............. & 57 & 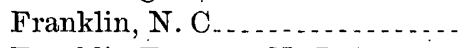 & 80 \\
\hline Corundum Hill, N. C. $21,30,37,80,83$ & 3,87 & Franklin Furnace, N. J . . . . . & 32 \\
\hline Cosalite $\ldots \ldots \ldots$ & 15 & Freieslebenite & 15 \\
\hline Covellite & 11 & Fuchsite $\ldots$ & 72 \\
\hline Mountains, Mont......... & 26 & Gadolinite $\ldots \ldots$ & 54 \\
\hline Creek, Colo _............ & 16 & Gahnite $\ldots \ldots$ & 20 \\
\hline Station, N. Y . . . . . . . & 78 & 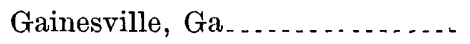 & 78 \\
\hline (n) & 17 & Gallatin Range, Mont_. . . . . . . . . . & 20 \\
\hline yllite $\ldots \ldots \ldots \ldots \ldots$ & 74 & Garnet $\ldots \ldots \ldots$ & 45,46 \\
\hline Costa Rica ........... & 35 . & Gearksutite .. & 18 \\
\hline te.............. & 80 & te. . . . . . . & 88 \\
\hline tite . . . . . . . . . . . & 14 & $\mathrm{wn}, \mathrm{N} . \mathrm{Mex} \ldots$ & 99 \\
\hline zite ........ & 99 & Glastonbury, Conn ......... & 111 \\
\hline County, Oreg ........... & 95 & Glanconite $\ldots \ldots \ldots$ & 89 \\
\hline County, Colo . . . . . . . . . . & 21 & Gold $\ldots \ldots \ldots \ldots$ & 10 \\
\hline (2. & 56 & Golden, Colo _. . $64,66,67,68,69$, & 70,91 \\
\hline te & 57 & n, Mass $\ldots \ldots \ldots$ & 45 \\
\hline (-1) & 57 & neur, N. Y. . . . . . & 61 \\
\hline lley, Cal . . . . . ...... & 95 & Grand Manan, New Brunswick. - & 69 \\
\hline rer, N. C . . . . . . . . . & 91 & Grant County, N. Mex ....... 1 & 13,106 \\
\hline$Y, \ldots \ldots \ldots$ & 61 & Granville, Mass . . . . . . . . & 84 \\
\hline Descloizite $\ldots \ldots \ldots \ldots$ & 99 & Mountain, Colo . . . . . . & 65 \\
\hline Devils Head Mountain, Colo _ _ 54,56 & 6,94 & Green River, N. C & 80,92 \\
\hline Diallage ........ & 32 & Talley, Cal . . . . . . . . & 83 \\
\hline$Y$ & 36 & $a l \ldots \ldots \ldots$ & 87 \\
\hline 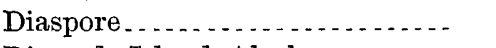 & 21 & Mountain, Mass . . . . . . & 26 \\
\hline Island, Alaska . . . . . . . . . & 38 & te & 98 \\
\hline Diopside $_{2}$ & 32 & Grossularite $\ldots \ldots \ldots$ & 45 \\
\hline Dolomite & 23 & Guitermanite $\ldots \ldots$ & $1 \overline{5}$ \\
\hline Dugway District, Utah.......... & 19 & Gunnison County, Col . . . 13, 14, & 15,65 \\
\hline Dumortierite $\ldots . . . \ldots \ldots \ldots$ & 58 & $m$ & 104 \\
\hline Eagle County, Colo & 108 & Gyrolite $\ldots \ldots \ldots$ & 64 \\
\hline Easton, $\mathrm{Pa} \ldots \ldots \ldots$ & & Haddam, Conn ....... & 62 \\
\hline Edenville, $\mathrm{N} . \mathrm{Y} \ldots \ldots \ldots$ & 96 & Halit & 17 \\
\hline $\mathrm{ds}, \mathrm{N} . \mathrm{Y} \ldots \ldots$ & 76 & - & 81 \\
\hline Elæolite $\ldots \ldots \ldots$ & 42 & rite $\ldots \ldots \ldots$ & 90 \\
\hline Elliott County, Ky $\ldots \ldots \ldots \ldots 45,47$ & 7,92 & Halls Valley, Colo . . . . . . . . . & 14 \\
\hline Elpasolite & 18 & Halotrichite $\ldots \ldots \ldots \ldots$ & 106 \\
\hline 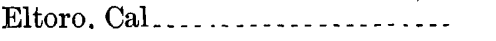 & 45 & Hamburg, N. J . . . . . . . . & 61 \\
\hline
\end{tabular}




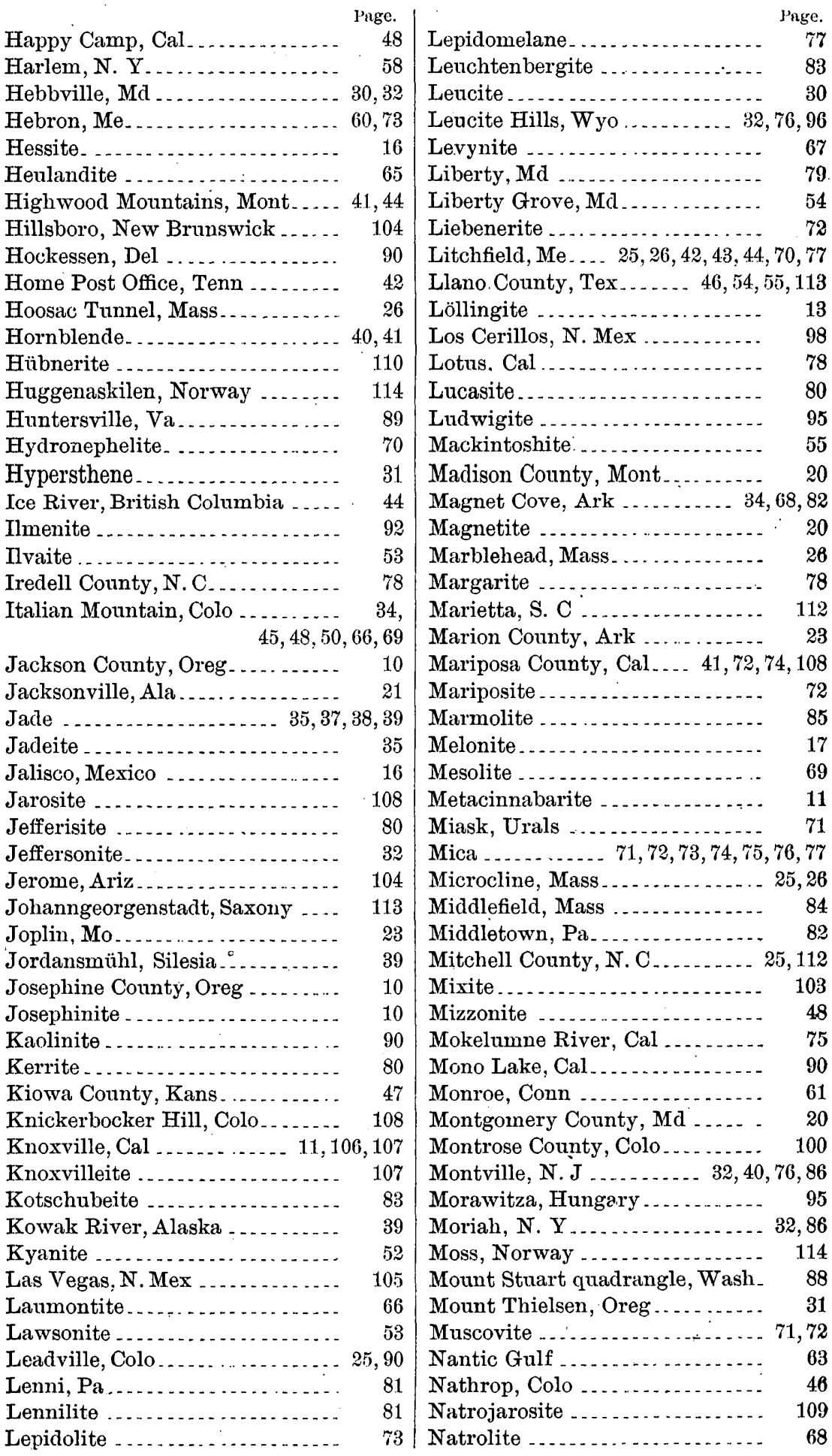


Nephelite.

Page.

Nephi, Utah

Nephrite

New Almaden, Cal.

$37,38,39$

Newburyport, Mass

11,64

New Idria, Cal

Newlin, $\mathrm{Pa}$

New York City

New Zealand

Niter

Nivenite

North Blandford, Mass

North Fowl Lake, Minn

Norway, Me

Nottingham, $\mathrm{Pa}$

73,91

Oaxaca, Mexico

Oligoclase

Olivenite

Olivine

Orford, N. H

Orthoclase

Ouray County, Colo ........... . 90,110

Owens Lake, Cal ................ . $\quad 24$

Pachnolite

Painterite

Palmer Center, Mass ............. 29, 40

Panderma, Island of . .......... 95

Pandermite.................. 95

Paris, $\mathrm{Me} \ldots \ldots \ldots \ldots$. . . . . . . . . . 62, 71, 73

Parkdale, Colo................ 28

Parrott City, Colo .............. 15

Parrsboro, N. S . . . . . . . . . 66,67,68

Paterson, N. J................ 51

Pectolite . .

Peru, $\mathrm{Me}$..................

Petalite .................... 25

Petzite . . . . . . . . . . . . . . . 16

Phippsburg, Me . . ............. 27, 50

Phlogopite .................. 76

Picrallumogene............... 105

Picrolite...................... 87

Piedmontite ................. 50

Pierrepont, N. Y ............. . 40,61

Pigeon Point, Minn.............. 26

Pikes Peak District, Colo ........ 17,

Pine Mountain $\mathrm{Md}$. $18,19,21,24,78$

$18,19,21,24,77$
50

Pisanite _.................... 105

Placerville, Colo ................. 78

Platte Mountain, Colo ..........

Pleonaste ...................... 20

Plumbojarosite ................ 109

Point Barrow, Alaska.

Poldnewaja River, Urals . . . . . . . 87

Polydymite ................... 12

Port Henry, N. Y . . . . . . . . . . . .

Powellite .................... 110

Prehnite... . . . . . . . . . . . . . . 51

Priceite ... . . . . . . . . . . . . . . 95

Prochlorite ... . . . . . . . . . . 84

Prosopite .... . . . . . . . . . . . . 19

Protovermiculite . . . . . . . .

Psilomelane .................. 22

Ptilolite ...................... 65

Pyrope ........................ 45

Pyrophyllite . . . . . . . . . . . . . . . . . 91

Pyroxene ..... . . . . . . . . . . . . . 32, 33, 34

Rabun County, Ga ............. 74

Rapid City, S. Dak_............. 98

Raymond, Me ................ 27

Red Hill, N. H . . . . . . . . . . . . . . 42

Redingtonite ................ 107

Red Mountain, Colo. . . . . . . . . . . 14

Rhodes Marsh, Nev ............. 95

Rico, Colo _.............. . . 90, 104, 108

Riddle, Oreg. . . . . . . . . . . . . . . 47.88

Robenhausen, Switzerland ...... 87

Rockport, Mass . . . . . . . . . . . . . 74, 77.

Rocky Hill, N. J . . . . . . . . . . . . . 82

Roscoelite ................... 78

Rowe, Mass . . . . . . . . . . . . . . . 85

Rowlandite ................... 55

Rumford, Me _... . . . . . . . . . 59, 60, 73

Running Water River, Wyo_.... 72

Russell, Mass . . . . . . . . . . . . . . 85

Russell, N. Y ... . . . . . . . . . .

Rutile ....................... 21

Saas Valley, Switzerland ....... 49

St. Michael, Alaska . . . . . . . . . . . . 38

St. Peters Dome, Colo _......... 17, 18, $19,21,71,90,93$

Salton, Cal .................... 17

Samarskite .................... 94

San Emigdio, Cal ............... $\quad 30$

San Juan County, Colo . . . . . . . . . 14, 15

Sardinal, Costa Rica ........... 35

Saussurite ..................... 49

Scolecite ... . . . . . . . . . . . . . 69

Scorodite ................... 103

Secret Canyon, Nev . . . . . . . . . . 103

Serpentine . . . . . . . . . . . 84, 85, $86,87,88$

Seven Devils District, Idaho . . . 46, 110

Shasta County, Cal ........... 49

Siderophyllite ................ 77

Sillimanite ................... 52

Silver Cliff, Colo . . . . . . . . . . 22, 33, 65 


\begin{tabular}{|c|c|}
\hline I & Page. \\
\hline Silver City, Idaho & Tremolite \\
\hline Silver Peak, Nev & Tres Cerritos Buttes, Cal \\
\hline Silverton, Colo.. & Triplite. \\
\hline Sinaloa, Mexico & Tscheffkinite \\
\hline kraatorp, Norway & Tuckahoe, N. Y . . \\
\hline Slatoust, Urals . . . . . . . . . . . . . 79,83 & Turquoise .......... \\
\hline Smithsonite .......... & Two Buttes, Colo . . . . . . ........... \\
\hline Soda Springs Valley, Nev & Tyrolite ......... \\
\hline Sodalite $\ldots \ldots \ldots \ldots$ & Tysonite. . . . . . . . \\
\hline Soda Niter. . . . . . . . . & Ulexite $\ldots \ldots \ldots$ \\
\hline South Mountain, Idaho .......... & Uraninite $\ldots \ldots \ldots \ldots \ldots 111,112,113,114$ \\
\hline Spessartite & Urao ........ \\
\hline Spinel ....... & Uvalde quadrangle, Tex ........ \\
\hline Staurolite .... & Valmont, Colo .. \\
\hline Sterling, N. J $\ldots \ldots \ldots$ & Vermiculite.... \\
\hline Stevenson Station, Md. . . . . . . . . & Vesuvianite.................. \\
\hline Stilbite $\ldots \ldots \ldots \ldots \ldots$ & Vesuvius $\ldots \ldots \ldots \ldots \ldots$ \\
\hline Stoneham, $\mathrm{Me} \ldots \ldots \ldots \ldots 52,72,97$ & Villeneuve, Canada \\
\hline Stony Point, N. C $\ldots \ldots \ldots \ldots \ldots \ldots$. . . . . 63,71 & Waluewite ........ \\
\hline Mass & Warrenite .......... \\
\hline Stromeyerite $\ldots . . . \ldots \ldots$ & Warwick, Mass . . . . . \\
\hline Sudbury, Canada $\ldots \ldots \ldots \ldots$ & Warwickite $\ldots \ldots \ldots$ \\
\hline ulphur Bank, Colo . . . . . . . . 88, 106 & Washington, D. C $\ldots \ldots, \ldots 40,49,84,92$ \\
\hline Table Mountain, Cal ........... 41 & Wassons Bluff, N.S....... $66,67,68$ \\
\hline Table Mountain, Colo ............ & Webster, Mass . . . . . . . . . . . . \\
\hline $66,67,68,69$ & Westchester, Pa ..... \\
\hline Talc & Wilmington, Del ... \\
\hline Talladega, Ala .............. & Winslow, Me ........ \\
\hline District, Colo . . . . . . . . . . . & Wollastonite . . . . . . . . . . . . \\
\hline Mountain, Colo ...... & ne \\
\hline (n. & Xanthophyllite $\ldots \ldots$ \\
\hline 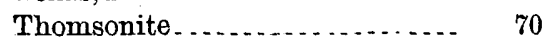 & Xenotime.............. \\
\hline Tiburon Peninsula, Cal . ....... & Yancey County, N. C .......... \\
\hline Tintic District, Utah . . . . . 101, 102, 103 & Yellowstone Park ......... 108, 106 \\
\hline Titanite & Yosemite Valley, Cal ... . . . . . . . . \\
\hline Tokajer-Berg, Hungary . . . . . . . . & Yttrialite ............. \\
\hline Tombstone, Ariz. $\ldots \ldots \ldots \ldots$ & Yucca.Station, Ariz . . . . . . . . \\
\hline Tooele County, Utah .... & Zinkenite... \\
\hline .......... & Zoisite .. \\
\hline$\ldots 59,60,61,62,63$ & Zunyite \\
\hline
\end{tabular}





\title{
PUBLICATIONS OF UNITED STATES GEOLOGICAL SURVEY.
}

\author{
[Bulletin No. 220.]
}

The serial publications of the United States Geological Survey consist of (1) Annual Reports, (2) Monographs, (3) Professional Papers, (4) Bulletins, (5) Mineral Resources, (6) Water-Supply and Irrigation Papers, (7) Topographic Atlas of the United States-folios and separate sheets thereof, (8) Geologic Atlas of the United States-folios thereof. The classes numbered 2, 7, and 8 are sold at cost of publication; the others are distributed free. A circular giving complete lists may be had on application.

The Bulletins, Professional Papers, and Water-Supply Papers treat of a variety of subjects, and the total number issued is large. They have therefore been classified into the following series: A, Economic geology: B, Descriptive geology; C, Systematic geology and paleontology; D, Petrography and mineralogy; E, Chemistry and physics; F, Geography; G, Miscellaneous; H, Forestry; I, Irrigation; $\mathrm{J}$, Water storage; K, Pumping water; L, Quality of water; M, General hydrographic investigations; $N$, Water power; $\mathrm{O}$, Underground waters; $\mathrm{P}$, Hydrographic progress reports. This paper is the twenty-fifth in Series D and the thirty-ninth in Series $\mathrm{E}$, the complete lists of which follow. $(\mathrm{B}=$ Bulletin, $\mathrm{PP}=$ Professional Paper. $)$

\section{SERIES D, PETROGRAPHY AND MINERALOGY.}

B 1. On hypersthene-andesite and on triclinic pyroxene in augitic rocks, by Whitman Cross, with a geological sketch of Buffalo Penks, Colorado, by S. F. Emmons. 1883.42 pp., 2 pls.

B 8. On secondary enlargements of mineral fragments in certain rocks, by R. D. Irving and C. R. Van Hise. 1884 . 56 pp., 6 pls. (Out of stock.)

B 12. A crystallographic study of the thinolite of Lake Lahontan, by E. S. Dana. $1884.34 \mathrm{pp} ., 3$ pls.

B 17. On the development of crystallization in the igneous rocks of Washoe, Nevadi with notes on the geology of the district, by Arnold Hague and J. P. Iddings. 1885 . $44 \mathrm{pp}$. (Ont of stock.)

B 20. Contributions to the mineralogy of the Rocky Mountains, by Whitman Cross and W. F. Hillebrand. 1885 . $114 \mathrm{pp} ., 1 \mathrm{pl}$. (Out of stock.)

B 28. The gabbros and associnted hornblende rocks occurring in the neighborhood of Baltimore, Maryland, by G. H. Williams. 1886.78 pp., 4 pls. (Out of stock.)

B 38. Peridotite of Elliott County, Kentucky, by J. S. Jiller. 1887. $31 \mathrm{pp} ., 1 \mathrm{pl}$

B 59. The gabbros and associated rocks in Delaware, by F. D. Chester. $1890.45 \mathrm{pp} ., 1 \mathrm{pl}$

$\mathrm{B}$ 61. Contributions to the minerulogy of the Pacitic coast, by W. H. Melville and Waldemar Lindgren. 1890. 40 pp., 3 pls.

B 62. The greenstone-schist arens of the Menominee and Marquette regions of Michigan: a contribution to the subject of dynamic metamorphism in eruptive rocks, by G. H. Williams; with introduction by R. D. Irving. $1890.241 \mathrm{pp}$., $16 \mathrm{pls}$. (Out of stock.)

$B$ 66. On a group of volcanic rocks from the Tewan Nountains, New Mexico, and on the occurrence of primary quartz in certain basalts, by J. P. Iddings. $1890.34 \mathrm{pp}$

$B$ 74. The minerals of North Carolina, by F. A. Genth. 1891 . $119 \mathrm{pp}$. (Out of stock.)

B 79. A late volcanic eruption in northern California and its peculiar lava, by J. S. Diller. 1891. 33 pp., 17 pls. (Out of stock.)

B 89. Some lava flows of the western slope of the Sierra Nevada, California, by F. L. Ransome. 1898. 74 pp., 11 pls.

B 107. The trip dikes of the Lake Champlain region, by J. F. Kemp and V. F. Masters. 1893.62 pp., 4 pls. (Out of stock.)

B 109. The eruptive and sedimentary rocks on Pigeon Point, Minnesota, and their contact phenomena, by W. S. Bayley. 1893 . 121 pp., 16 pls.

B 126. A mineralogical lexicon of Franklin, Hampshire, and Hampden counties, Massachusetts, by B. K. Emerson. 1895.180 pp., 1 pl.

B 136. Volcanic rocks of South Molutain, Pennsylyania, by Florence Bascom, 1896, $124 \mathrm{pp}, 28 \mathrm{pls}$

B 150. The educational series of rock specimens collected and distributed by the United States Geological Survey, by J. S. Diller. 1898. 400 pp., 47 pls.

B 157. The gneisses, gabbro-schists, and associated rocks of southwestern Minnesota, by C. W. Hall. 1899. 160 pp., 27 pls.

PP 3. Geology and petrography of Crater Lake National Park, by J. S. Diller and H. B. Patton. 1902. 167 pp., 19 pls.

B 209. The geology of Ascutney Mountain, Vermont, by R. A. Daly. 1.903 .122 pp. $7 \mathrm{pls}$

PP 14. Chemical analyses of igneous rocks published from 1884 to 1900 , with a critical discussion of the character and use of analyses, by H. S. Washington. $1903.495 \mathrm{pp}$.

PP 18. Chemical composition of igneous rocks expressed by means of diagrams, with reference to rock classification on a quantitative chemico-mineralogical basis, by J. P. Iddings. 1903. 98 pp. 8 pls.

B 220. Mineral analyses from the laboratories of the United States Geological Survey, 1880 to 1903, tabulated by F. W. Clarke, chief chemist. 1903 . 119 pp. 


\section{SERIES E, CHEMISTRY AND PHYSICS.}

B 9. Report of work done in the Washington laboratory during the fiscal year 1883-84, by F. W. Clarke and T. M. Chatard. $1884.40 \mathrm{pp}$. (Out of stock.)

$B$ 14. Electrical and inagnet $c$ properties of the iron carburets, by Carl Barus and Vincent Strouhal. 1885. $238 \mathrm{pp.}$ (Out of stock.)

B 27. Report of work done in the Division of Chemistry and Physics, mainly during the year 1884-85. 1886. $80 \mathrm{pp}$

B 32. Lists and analyses of the mineral springs of the United States (a preliminary study), by AIbert C. Peale. 1886. $235 \mathrm{pp}$. (Out of stock.)

B 35. Physical properties of the iron arburets, by Carl Barus and Vincent Strouhal. $1886.62 \mathrm{pp}$.

B 36. Subsidence of fine solid particles in liquids, by Carl Barus. 1886 . $58 \mathrm{pp}$. (Out of stock.)

B 42. Report of work done in the Division of Chemistry and Physics, mainly during the fiscal year $1885-86$, by F. W. Clarke. 1887 . $152 \mathrm{pp} ., 1 \mathrm{pl}$. (Out of stock.)

B 47. Analyses of waters of the Yellowstone National Park, with an account of the methods of analyses employed, by Frank Austin Goo. h and James Ed ward Whitfield. 1888. 84 pp. (Out of stock.)

B 52. Subaerial decay of rocks and origin of the red color of certain formations, by Israel Cook Russell. 1889 . $65 \mathrm{pp}$., 5 pls. (Out of stock.)

B 54. On the thermoelectric measurement of high temperatures, by Carl Barus. $1889.313 \mathrm{pp} ., 11 \mathrm{pls}$. (Out of stock.)

B 55. Report of work done in the Division of Chemistry and Physics, mainly during the fiseal year $1886-87$, by F. W. Clarke. 1889.96 py. (Out of stock.)

B 60. Report of work done in the Division of Chemistry and Physics, mainly during the fiscal year $1887-88$. 1890. $174 \mathrm{pp.}$ (Out of stock.)

B 64. Report of work done in the Division of Chemistry and Physics, mainly during the fiscal year $1888-89$, by F. W. Clarke. 1890.60 pp.

B 68. Earthquakes in California in 1889, by James Edward Keeler. 1890. 25 pp.

B 73. Thíe viscosity of solids, by Carl Barus. 1891 . xii, $139 \mathrm{pp}$., $6 \mathrm{pls}$.

B 78. Report of work done in the Division of Chemistry and Physics, mainly during the fiscal year $1889-90$, by F. W. Clarke. 1891. $131 \mathrm{pp}$

B 90. Report of work done in the Division of Chemistry and Physics, mainly during the fiscal year $1890-91$, by F. W. Clarke. $1892.77 \mathrm{pp}$.

B 92. The compressibility of liquids, by Carl Barus. $1892.96 \mathrm{pp} ., 29 \mathrm{pls}$

B 94. The mechanism of solid viscosity, by Carl Barus. $1892.138 \mathrm{pp}$.

B 95. Earthquakes in California in 1890 and 1891, by Edward Singleton Holden. 1892. $31 \mathrm{pp}$.

B 96: The volume thermodynamics of liquids, by Carl Barus. $1892.100 \mathrm{pp}$.

B 103. High temperature work in igneous fusion and ebullition, chiefly in relation to pressure, by Carl Barus. 1893. 57 pp., 9 pls.

B 112. Earthquakes in California in 1892 , by Charles D. Perrine. $1893.57 \mathrm{pp}$

B 113. Report of work done in the Division of Chemistry and Physics during the fiscal years 1891-92 and $1892-93$, by F. W. Clarke. $1893.115 \mathrm{pp}$

B 114. Earthquakes in California in 1893 , by Charles D. Perrine. 1894. $23 \mathrm{pp}$

B 125. The constitution of the silicates, by F. WV. Clarke. $1895.100 \mathrm{pp}$. (Out of stock.)

B 129. Earthquakes in California in 1894, by Charles D. Perrine. 1895. $25 \mathrm{pp}$.

B 147. Earthquakes in California in 1895, by Charles D. Perrine. 1896. $23 \mathrm{pp}$

B 148. Analyses of rocks, with a chapter on analytical methods, laboratory of the United States Geological Survey, 1880 to 1896 , by F. W. Clarke and W. F. Hillebrand. 1897. $306 \mathrm{pp}$. (Out of stock.)

B 155. Earthquakes in California in 1896 and 1897, by Charles D. Perrine. 1898. 47 pp.

B 161. Earthquakes in Califorinia in 1898, by Charles D. Perrine. $1899.31 \mathrm{pp} ., 1 \mathrm{pl}$.

B 167. Contributions to chemistry and mineralogy from the laboratory of the United States Geological Survey; Frank W. Clarke, chief chemist. $1900.166 \mathrm{pp}$.

B 168. Analyses of rocks, laboratory of the United States Geological Survey, 1880 to 1899, tabulated by F. W. Clarke. $1900.308 \mathrm{pp}$. (Out'of stock.)

B 176. Some principles and methods of rock analysis, by W. F. Hillebrand. $1900.114 \mathrm{pp}$.

B 186. On pyrite and marcasite, br H. N. Stokes. 1900 . $50 \mathrm{pp}$

B 207: The action of ammonium chloride upon silicates, by F. W. Clarke and George Steiger. 1902. $57 \mathrm{pp}$. (Out of stock.)

PP 14. Chemical analyses of igneous rocks published from 1884 to 1900 , with a critical discussion of the character and use of analyses, by H. S. Washington. $1903.495 \mathrm{pp}$

PP 18. Chemical composition of igneolis rocks expressed by means of diagrams, with reference to rock classification on a quantitative chemico-mineralogical basis, by J. P. Iddings. 1903. 98 pp., 8 pls.

B 220. Mineral analyses from the laboratories of the United States Geological Survey, 1880 to 1903 , tabulated by F. W. Clarke, chief chemist. $1903.119 \mathrm{pp}$.

Correspondence should be addressed to-

\section{The Director,}

United States Geological Survet,

November, 1903.

WASHINGTON, D. C. 


\section{LIBRARY CATALOGUE SLIPS.}

[Mount each slip upon a separate card, placing the subject at the top of the second slip. The name of the series should not be repeated on the series card, but the additional numbers should be added, as received, to the first entry.]

\section{Clarke, $\mathrm{F}$ [rank] $\mathrm{W}$ [igglesworth].}

... Mineral analyses from the laboratories of the - United States Geological survey, I880 to I903, tabulated by F. W. Clarke, chief chemist. Washington, Gov't print. off., 1903.

119, III p. 231 $2_{2}^{\text {crn }}$. (U. S. Geological survey. Bulletin no. 220.)

Subject series D, Petrography and mineralogy, 25; E, Chemistry and. physics, 39 .

\section{Clarke, F [rank] W [igglesworth].}

... Mineral analyses from the laboratories of the United States Geological survey, I880 to I903, tabulated by F. W. Clarke, chief chemist. Washington, Gov't print. off., I903.

119, III p. 231 $2_{2}^{\mathrm{cm}}$. (U. S. Geological survey. Bulletin no. 220.)

Subject series D, Petrography and mineralogy, 25; E, Chemistry and physics, 39.

\section{U. S. Geological survey.}

Bulletins.

no. 220. Clarke, F. W. Mineral analyses from the laboratories of the U. S. Geological survey, r880-1903. 1903 .

\section{U. S. Dept. of the Interior.}

see also

U. S. Geological survey. 